\title{
THE BERNSTEIN CENTER OF THE CATEGORY OF SMOOTH $W(k)\left[G_{n}(F)\right]-M O D U L E S$
}

\author{
DAVID HELM \\ Department of Mathematics, Imperial College London, 180 Queen's Gate, \\ London SW7 2AZ, UK; \\ email: dhelm@imperial.ac.uk
}

Received 16 January 2015; accepted 21 April 2016

\begin{abstract}
We consider the category of smooth $W(k)\left[\operatorname{GL}_{n}(F)\right]$-modules, where $F$ is a $p$-adic field and $k$ is an algebraically closed field of characteristic $\ell$ different from $p$. We describe a factorization of this category into blocks, and show that the center of each such block is a reduced, $\ell$-torsion free, finite type $W(k)$-algebra. Moreover, the $k$-points of the center of a such a block are in bijection with the possible 'supercuspidal supports' of the smooth $k\left[\mathrm{GL}_{n}(F)\right]$-modules that lie in the block. Finally, we describe a large explicit subalgebra of the center of each block and give a description of the action of this algebra on the simple objects of the block, in terms of the description of the classical 'characteristic zero' Bernstein center of Bernstein and Deligne [Le 'centre' de Bernstein, in Representations des groups redutifs sur un corps local, Traveaux en cours (ed. P. Deligne) (Hermann, Paris), 1-32].
\end{abstract}

2010 Mathematics Subject Classification: 11F33, 11F70 (primary); 22E50 (secondary)

\section{Introduction}

The center of an abelian category $\mathcal{A}$ is the endomorphism ring of the identity functor of that category. It is a commutative ring that acts naturally on every object of $\mathcal{A}$, a fact which often allows one to approach questions about $\mathcal{A}$ from a moduletheoretic point of view.

One spectacular success of this approach originates with work of BernsteinDeligne [BD], who computed the centers of categories of smooth complex representations of $p$-adic algebraic groups. The center of such a category is called the Bernstein center. Bernstein and Deligne give a factorization of this category

(C) The Author 2016. This is an Open Access article, distributed under the terms of the Creative Commons Attribution licence (http://creativecommons.org/licenses/by/4.0/), which permits unrestricted re-use, distribution, and reproduction in any medium, provided the original work is properly cited. 
into blocks, known as Bernstein components, as well as a simple and explicit description of the center of each block, which is a finite type $\mathbb{C}$-algebra. In particular, they showed that the $\mathbb{C}$-points of the Bernstein center were in bijection with the supercuspidal supports of irreducible smooth complex representations.

The results of [BD] made it possible to give purely algebraic proofs of theorems about smooth representations that previously could only be proven via deep results from Fourier theory; Bushnell-Henniart's results about Whittaker models in $[\mathbf{B H}]$ are an example of this approach.

In recent years there has been considerable interest in studying smooth representations over fields other than the complex numbers, or even over more general rings. To apply similar techniques in such a setting one needs to understand the centers of categories of smooth representations over $\mathbb{F}_{\ell}$ or $\mathbb{Z}_{\ell}$ (or even over $\mathbb{Z}$ ). Some progress along these lines was made by Dat [D2]; in particular he was able to give an explicit description of the center of the category of smooth representations of a $p$-adic algebraic group $G$ over $\mathbb{Z}_{\ell}$, for $\ell$ a banal prime; that is, for $\ell$ prime to the order of $G\left(\mathbb{F}_{p}\right)$.

More recently Paskunas [Pa] has studied the center of a category of representations of $\mathrm{GL}_{2}\left(\mathbb{Q}_{p}\right)$ over $\mathbb{F}_{p}$; his results allow him to characterize the image of the Colmez functor.

We fix our attention on the category $\operatorname{Rep}_{W(k)}\left(\mathrm{GL}_{n}(F)\right)$ of smooth representations of $\mathrm{GL}_{n}(F)$, where $F$ is a $p$-adic field, over a ring of Witt vectors $W(k)$, for $k$ an algebraically closed field of characteristic $\ell$ different from $p$. We obtain a factorization of this category into blocks that parallels the Bernstein decomposition over $\mathbb{C}$. This description is closely related to the decomposition due to Vigneras [V2] of the category of smooth representations of $\mathrm{GL}_{n}(F)$ over $k$; in both decompositions the blocks are parameterized by inertial equivalence classes of pairs $(L, \pi)$, where $L$ is a Levi subgroup of $\mathrm{GL}_{n}(F)$ and $\pi$ is an irreducible supercuspidal representation of $L$ over $k$. Indeed, our approach owes a substantial amount to the ideas of Vigneras, and in particular her use of $G$-covers in an integral setting. In spite of this we do not rely on Vingeras's Bernstein decomposition over $k$ [V2], and indeed our description of the Bernstein decomposition over $W(k)$ implies the result of Vigneras over $k$.

More precisely, we introduce (Definition 4.12) a notion of mod $\ell$ inertial supercuspidal support for simple objects $\Pi$ in $\operatorname{Rep}_{W(k)}\left(\operatorname{GL}_{n}(F)\right)$. When $\Pi$ is killed by $\ell$ this is just the usual notion of supercuspidal support (taken up to inertial equivalence), but this notion also makes sense for those $\Pi$ on which multiplication by $\ell$ is an isomorphism. For an inertial equivalence class $(L, \pi)$ as above, we can then consider the full subcategory $\operatorname{Rep}_{W(k)}\left(\mathrm{GL}_{n}(F)\right)_{[L, \pi]}$ whose objects are those $\Pi$ such that every simple subquotient of $\Pi$ has $\bmod \ell$ inertial supercuspidal support given by $(L, \pi)$. Our first main result, Theorem 11.8, 
then states that the blocks of $\operatorname{Rep}_{W(k)}\left(\mathrm{GL}_{n}(F)\right)$ are precisely the subcategories $\operatorname{Rep}_{W(k)}\left(\mathrm{GL}_{n}(F)\right)_{[L, \pi]}$.

For each such $(L, \pi)$, let $A_{[L, \pi]}$ be the center of $\operatorname{Rep}_{W(k)}\left(\operatorname{GL}_{n}(F)\right)_{[L, \pi]}$. The main objective of this paper is to understand the structure of $A_{[L, \pi]}$ and its action on the objects of $\operatorname{Rep}_{W(k)}\left(\mathrm{GL}_{n}(F)\right)_{[L, \pi]}$. Our starting point is the BernsteinDeligne description of the analogous question over algebraically closed fields. Indeed, if $\overline{\mathcal{K}}$ is the algebraic closure of the field of fractions of $W(k)$, then tensoring with $\overline{\mathcal{K}}$ defines a functor from $\operatorname{Rep}_{W(k)}\left(\mathrm{GL}_{n}(F)\right)$ to $\operatorname{Rep}_{\overline{\mathcal{K}}}\left(\mathrm{GL}_{n}(F)\right)$; the image of this functor is a product of blocks of $\operatorname{Rep}_{\overline{\mathcal{K}}}\left(\mathrm{GL}_{n}(F)\right)$.

More precisely, Bernstein and Deligne show that the blocks of $\operatorname{Rep}_{\overline{\mathcal{K}}}\left(\mathrm{GL}_{n}(F)\right)$ are indexed by inertial equivalence classes of pairs $(M, \tilde{\pi})$, where $\tilde{\pi}$ is an irreducible supercuspidal representation of $\mathrm{GL}_{n}(F)$ over $\overline{\mathcal{K}}$. If we denote the corresponding block by $\operatorname{Rep}_{\overline{\mathcal{K}}}\left(\mathrm{GL}_{n}(F)\right)_{M, \tilde{\pi}}$, then the essential image of the block $\operatorname{Rep}_{\overline{\mathcal{K}}}\left(\mathrm{GL}_{n}(F)\right)_{[L, \pi]}$ under the functor that tensors with $\overline{\mathcal{K}}$ is the product of those $\operatorname{Rep}_{\overline{\mathcal{K}}}\left(\mathrm{GL}_{n}(F)\right)_{M, \tilde{\pi}}$ whose objects, when considered as $W(k)\left[\mathrm{GL}_{n}(F)\right]$-modules, are objects of $\operatorname{Rep}_{W(k)}\left(\mathrm{GL}_{n}(F)\right)_{[L, \pi]}$. It is then not hard to show (Proposition 12.1) that in this case one has an isomorphism of $\overline{\mathcal{K}}$-algebras:

$$
A_{[L, \pi]} \otimes \overline{\mathcal{K}} \cong \prod_{M, \tilde{\pi}} A_{M, \tilde{\pi}},
$$

where $A_{M, \tilde{\pi}}$ is the center of $\operatorname{Rep}_{\overline{\mathcal{K}}}\left(\mathrm{GL}_{n}(F)\right)_{M, \tilde{\pi}}$. Since (for formal reasons) $A_{[L, \pi]}$ is $\ell$-torsion free, this shows that $A_{[L, \pi]}$ embeds naturally as a $W(k)$-subalgebra of the product of the $A_{M, \tilde{\pi}}$. The Bernstein-Deligne theory (which we summarize in Section 3 ) gives a completely explicit description of the latter.

Understanding the integral structure of $A_{[L, \pi]}$ is considerably more difficult; our approach is to construct explicit projective objects $\mathcal{P}_{K, \tau}$ of $\operatorname{Rep}_{W(k)}\left(\mathrm{GL}_{n}(F)\right)$ from maximal distinguished cuspidal $k$-types $(K, \tau)$. These projectives have a number of useful properties; in particular their endomorphism rings are commutative, and can be explicitly computed after tensoring with $\overline{\mathcal{K}}$. We carry out this computation in Sections 6 (in the depth zero case) and 8 (for the general case). Although the integral structure of the endomorphism ring is harder to describe, we are able to construct an explicit subalgebra $C_{K, \tau}$ of the endomorphism ring of $\mathcal{P}_{K, \tau}$ in Sections 7 and 9, and describe the action of this subalgebra explicitly. We also show, in Section 10, that the action of $C_{K, \tau}$ makes $\mathcal{P}_{K, \tau}$ into an admissible $C_{K, \tau}\left[\mathrm{GL}_{n}(F)\right]$-module.

As a result of this admissibility, we are able to deduce Bernstein's second adjointness for the group $\mathrm{GL}_{n}(F)$, a fact which allows us to build further projective modules out of the $\mathcal{P}_{K, \tau}$ via parabolic induction. In this way, we construct, for each block $\operatorname{Rep}_{W(k)}\left(\mathrm{GL}_{n}(F)\right)_{[L, \pi]}$, a faithfully projective object $\mathcal{P}_{[L, \pi]}$. 
From the fact that $\mathcal{P}_{[L, \pi]}$ is faithfully projective, we deduce (see Section 12) that an element of $A_{[L, \pi]} \otimes_{W(k)} \overline{\mathcal{K}}$ lies in $A_{[L, \pi]}$ if, and only if, it preserves $\mathcal{P}_{[L, \pi]}$ in $\mathcal{P}_{[L, \pi]} \otimes_{W(k)} \overline{\mathcal{K}}$. The module $\mathcal{P}_{[L, \pi]}$ is a direct sum of modules built by parabolic induction from tensor products of $\mathcal{P}_{K, \tau}$. We therefore have a wide array of endomorphisms of $\mathcal{P}_{[L, \pi]}$ at our disposal, because of our explicit understanding of the endomorphism algebras $C_{K, \tau}$. In Section 12, we exploit this to construct a subalgebra $C_{[L, \pi]}$ of the endomorphism ring of $\mathcal{P}_{[L, \pi]}$, and we show that this subalgebra actually lies in $A_{[L, \pi]}$. Moreover, we can show that $\mathcal{P}_{[L, \pi]}$ is admissible over $C_{[L, \pi]}$, and thus that $A_{[L, \pi]}$ is a finitely generated $C_{[L, \pi]}$-module.

A consequence of this finiteness is that an object $\Pi$ of $\operatorname{Rep}_{W(k)}\left(\operatorname{GL}_{n}(F)\right)_{[L, \pi]}$ is admissible as an $A_{[L, \pi]}\left(\mathrm{GL}_{n}(F)\right)$-module if, and only if, it is finitely generated as a $W(k)\left[\mathrm{GL}_{n}(F)\right]$-module. This is an integral analogue of a result of Bernstein over fields of characteristic zero.

Our construction of $\mathcal{P}_{[L, \pi]}$ also allows us to prove results describing the interaction between the ring $A_{[L, \pi]}$ and parabolic induction (we refer the reader to Theorem 12.3 for a precise statement). This also allows us to show that $A_{[L, \pi]}$ admits a tensor factorization as a tensor product of rings $A_{\left[L_{i}, \pi_{i}\right]}$, where each $\left(L_{i}\right.$, $\pi_{i}$ ) is a so-called simple pair. (Theorem 12.4.)

The action of $C_{[L, \pi]}$ on irreducible representations of $\mathrm{GL}_{n}(F)$, both in characteristic zero and in characteristic $\ell$, can be made completely explicit in terms of a choice of certain 'compatible systems of cuspidals'. (We refer the reader to Theorem 9.4 and the discussion preceding it for a description of these systems.) From this one can show that the action of $C_{[L, \pi]}$ on irreducible smooth $k$-representations of $\mathrm{GL}_{n}(F)$ in $\operatorname{Rep}_{W(k)}\left(\mathrm{GL}_{n}(F)\right)_{[L, \pi]}$ is sufficient to distinguish representations with different supercuspidal supports (Proposition 12.11). On the other hand, it is not hard to show that if two irreducible $k$-representations of $\mathrm{GL}_{n}(F)$ have the same supercuspidal support, then $A_{[L, \pi]}$ acts on both of them via the same map $A_{[L, \pi]} \rightarrow k$. In particular, the $k$-points of both $\operatorname{Spec} C_{[L, \pi]}$ and Spec $A_{[L, \pi]}$ are in natural bijection with the set of possible supercuspidal supports of irreducible $k$-representations of $\mathrm{GL}_{n}(F)$ in the block corresponding to $(L, \pi)$. (Corollary 12.12.) This gives a ' $\bmod \ell$ ' analogue of the corresponding result of Bernstein-Deligne for complex points of the classical Bernstein center. An immediate corollary is that the map $\operatorname{Spec} A_{[L, \pi]} / \ell \rightarrow \operatorname{Spec} C_{[L, \pi]} / \ell$ is a bijection on $k$-points; from this we can conclude that the inclusion of $C_{[L, \pi]} / \ell$ in $A_{[L, \pi]} / \ell$ identifies $C_{[L, \pi]} / \ell$ with the reduced quotient $\left(A_{[L, \pi]} / \ell\right)^{\mathrm{red}}$.

In the final section, we prove some additional results about the structure of the endomorphism rings of the projectives $\mathcal{P}_{K, \tau}$, assuming a result (Theorem 13.1) whose proof we postpone to the sequel paper [H1]. Conditionally on this result, we are able to realize $A_{[L, \pi]}$ as a subalgebra of a much simpler ring than the endomorphism ring of $\mathcal{P}_{[L, \pi]}$; this simpler ring can, in small cases, be given a 
completely explicit description. As a result we are able to give completely explicit descriptions of $A_{[L, \pi]}$ in numerous situations where $n$ is small relative to $\ell$ and the order of $q$ modulo $\ell$. (See the examples at the end of Section 13 for precise statements.)

This is the first paper in a four-part series. The second paper, [H1], applies the structure theory of $A_{[L, \pi]}$ developed here to questions that arise from the theory of Whittaker models, that were first studied over the complex numbers in $[\mathbf{B H}]$. We establish versions of several of these results that hold for smooth representations of $\mathrm{GL}_{n}(F)$ over $W(k)$. These results have implications for the structure theory of certain representations associated to the 'local Langlands correspondence in families' of [EH]. In particular, $[\mathbf{E H}]$ conjectures the existence of certain algebraic families of admissible representations of $\mathrm{GL}_{n}(F)$ attached to Galois representations. These families are characterized by certain properties that can be understood in terms of the spaces of Whittaker functions we consider. We make use of this in [H1] to reduce the question of the existence of such families to a natural conjecture that relates $A_{[L, \pi]}$ to the deformation theory of Galois representations, via the local Langlands correspondence.

The third and fourth papers in the series, [H2] and [HM], will be devoted to exploring this relationship between $A_{[L, \pi]}$ and Galois theory. In particular, we construct a natural isomorphism between the completion of $A_{[L, \pi]}$ at a point $x$ as a subalgebra of the universal framed deformation ring of the semisimple representation of $G_{F}$ attached to $x$ via local Langlands. In particular, this will yield a proof of the existence of the families conjectured in $[\mathbf{E H}]$.

\section{Faithfully projective modules and the Bernstein center}

Definition 2.1. Let $\mathcal{A}$ be an abelian category. The center of $\mathcal{A}$ is the ring of endomorphisms of the identity functor Id $: \mathcal{A} \rightarrow \mathcal{A}$. More prosaically, an element of $\mathcal{A}$ is a choice of element $f_{M} \in \operatorname{End}(M)$ for every object $M$ in $\mathcal{A}$, satisfying the condition $f_{M} \circ \phi=\phi \circ f_{N}$ for every morphism $\phi: N \rightarrow M$ in $\mathcal{A}$.

If $\mathcal{A}$ is the category of right $R$-modules for some (not necessarily commutative) ring $R$, then it is easy to see that the center of $\mathcal{A}$ is the center $Z(R)$ of the ring $R$. Indeed, $Z(R)$ acts on every object of $\mathcal{A}$; this defines a map of $Z(R)$ into the center. Its inverse is constructed by considering the action of $Z(R)$ on $R$, considered as a right $R$-module.

When $\mathcal{A}$ is a more general abelian category, we can often describe its center by reducing to the case of a module category. We more or less follow the ideas of [R, Section 1.1]. The key is to find an object in $A$ that is faithfully projective, in the following sense: 
Definition 2.2. Let $\mathcal{A}$ be an abelian category with direct sums. An object $P$ in $\mathcal{A}$ is faithfully projective if:

(1) $P$ is a projective object of $\mathcal{A}$.

(2) The functor $M \mapsto \operatorname{Hom}(P, M)$ is faithful.

(3) $P$ is small; that is, one has an isomorphism:

$$
\bigoplus_{i \in I} \operatorname{Hom}\left(P, M_{i}\right) \cong \operatorname{Hom}\left(P, \oplus_{i \in I} M_{i}\right)
$$

for any family $M_{i}$ of objects of $\mathcal{A}$ indexed by a set $I$.

One checks easily that the condition that $M \mapsto \operatorname{Hom}(P, M)$ is faithful is equivalent to the condition that $\operatorname{Hom}(P, M)$ is nonzero for every object $M$ of $\mathcal{A}$. If $\mathcal{A}$ has the property that every object of $\mathcal{A}$ has a simple subquotient, then it suffices to check that $\operatorname{Hom}(P, M)$ is nonzero for every simple $M$.

If $P$ is a faithfully projective object of $\mathcal{A}$, one has:

Proposition 2.3 [R, Theorem 1.1]. Let $P$ be faithfully projective. The functor $M \mapsto \operatorname{Hom}(P, M)$ is an equivalence of categories from $\mathcal{A}$ to the category of right $\operatorname{End}(P)$-modules. In particular, the center of $\mathcal{A}$ is isomorphic to the center of $\operatorname{End}(P)$.

Idempotents of the center correspond to factorizations of $\mathcal{A}$ as a product of categories. In practice we can obtain these factorizations by constructing suitable injective objects of $\mathcal{A}$.

Proposition 2.4. Suppose that every object of $\mathcal{A}$ has a simple subquotient, let $S$ be a subset of the simple objects of $\mathcal{A}$, and let $I_{1}, I_{2}$ be injective objects of $\mathcal{A}$ such that, up to isomorphism:

(1) every simple subquotient of $I_{1}$ is in $S$;

(2) every object in $S$ is a subobject of $I_{1}$;

(3) no simple subquotient of $I_{2}$ is in $S$; and

(4) every simple object of $\mathcal{A}$ that is not in $S$ is a subobject of $I_{2}$.

Then every object $M$ of $\mathcal{A}$ splits canonically as a product $M_{1} \times M_{2}$, where every simple subquotient of $M_{1}$ is in $S$, and no simple subquotient of $M_{2}$ is in $S$. This gives a decomposition of $\mathcal{A}$ as a product of the full subcategories $\mathcal{A}_{1}$ and $\mathcal{A}_{2}$ 
of $\mathcal{A}$, where the objects of $\mathcal{A}_{1}$ are those objects $M_{1}$ of $\mathcal{A}$ such that every simple subquotient of $M_{1}$ is in $S$, and the objects of $\mathcal{A}_{2}$ are those objects $M_{2}$ of $\mathcal{A}$ such that no simple subquotient of $M_{2}$ is in $S$. Moreover, every object of $\mathcal{A}_{1}$ has an injective resolution by direct sums of copies of $I_{1}$, and every object of $\mathcal{A}_{2}$ has an injective resolution by direct sums of copies of $I_{2}$.

Proof. Let $M_{1}$ be the maximal quotient of $M$ such that every simple subquotient of $M_{1}$ is in $S$, and let $M_{2}$ be the kernel of the map $M \rightarrow M_{1}$. We first show $\operatorname{Hom}\left(M_{2}, I_{1}\right)=0$. Suppose we have a nonzero map of $M_{2}$ into $I_{1}$, with kernel $N$. Then the injection of $M_{2} / N$ into $I_{1}$ would extend to an injection of $M / N$ into $I_{1}$, and thus $M / N$ would be a quotient of $M$, dominating $M_{1}$, all of whose simple subquotients were in $S$. It follows that no simple subquotient of $M_{2}$ lies in $S$, as such a subquotient would yield a nonzero map of $M_{2}$ to $I_{1}$.

If we let $M_{3}$ be the maximal quotient of $M$ such that no simple subquotient of $M_{3}$ is in $S$, then the same argument (with $I_{1}$ and $I_{2}$ reversed) shows that every simple subquotient of the kernel of the map $M \rightarrow M_{3}$ lies in $S$. In particular the projection of $M_{2}$ onto $M_{3}$ is injective. Suppose the image of $M_{2}$ were not all of $M_{3}$. Then (as $M$ surjects onto $M_{3}$ ), there is a simple subquotient of $M_{3}$ that is also a subquotient of $M / M_{2}$; such an object would have to be in both $S$ and its complement. Thus $M_{2}$ is isomorphic to $M_{3}$, and hence $M$ splits, canonically, as a product $M_{1} \times M_{2}$. The decomposition of $\mathcal{A}$ as the product $\mathcal{A}^{\prime} \times \mathcal{A}^{\prime \prime}$ is now immediate, as is the claim about resolutions.

REMARK 2.5. It is easy to make a dual argument with projective objects; we state the proposition in terms of injectives because that is the form of the proposition we will use.

\section{The Bernstein center of $\operatorname{Rep}_{\overline{\mathcal{K}}}(G)$}

Let $G=\mathrm{GL}_{n}(F)$ be a general linear group over a $p$-adic field $F$, and let $k$ be an algebraically closed field of characteristic $\ell$ not equal to $p$. Our goal is to study the Bernstein center of the category $\operatorname{Rep}_{W(k)}(G)$ of smooth $W(k)[G]$-modules. It will be convenient for us to assume throughout that $\ell$ is odd, so that $W(k)$ necessarily contains a square root of $q$, where $q$ is the order of the residue field of $F$. This is done largely for convenience; when $\ell$ is 2 all the arguments we present remain valid after adjoining a square root of $q$ to $W(k)$. We begin by studying the category $\operatorname{Rep}_{\overline{\mathcal{K}}}(G)$ of smooth $\overline{\mathcal{K}}[G]$-modules, where $\mathcal{K}$ is the field of fractions of $W(k)$. Most of the results of this section are standard. We limit ourselves to the case of $\mathrm{GL}_{n}(F)$, although the results of this section have analogues for a general reductive group. 
The description of the center of $\operatorname{Rep}_{\overline{\mathcal{K}}}(G)$ depends heavily on the theory of parabolic induction, and particularly the notions of cuspidal and supercuspidal support, which we now recall. Let $(M, \pi)$ be an ordered pair consisting of a Levi subgroup $M$ of $G$ and an absolutely irreducible cuspidal representation $\pi$ of $M$.

Let $P$ be a parabolic subgroup of $G$, with Levi subgroup $M$ and unipotent radical $U$, and let $\pi=\pi_{1} \otimes \cdots \otimes \pi_{r}$ be a $W(k)[M]$-module. We let $i_{P}^{G}$ be the normalized parabolic induction functor of [BZ]; that is, $i_{P}^{G} \pi$ is the $W(k)[G]$ module obtained by extending $\pi$ by a trivial $U$-action to a representation of $P$, twisting by a square root of the modulus character of $P$, and inducing to $G$. (This depends on a choice of square root of $q$ in $W(k)$; we fix such a choice once and for all.) Similarly, we denote by $r_{G}^{P}$ the parabolic restriction functor from $W(k)[G]$ modules to $W(k)[M]$-modules.

DEFINITION 3.1. Let $M$ be a Levi subgroup of $G$, and let $\pi$ be an absolutely irreducible supercuspidal representation of $M$ over a field $L$. Let $\Pi$ be an absolutely irreducible representation of $G$ over $L$. Then the pair $(M, \pi)$ belongs to the supercuspidal support of $\Pi$ if there exists a parabolic subgroup $P$ of $G$, with Levi subgroup $M$, such that $\Pi$ is isomorphic to a Jordan-Hölder constituent of the normalized parabolic induction $i_{P}^{G} \pi$.

Definition 3.2. Let $M$ be a Levi subgroup of $G$, and let $\pi$ be a cuspidal representation of $M$ over a field $L$. Let $\Pi$ be an absolutely irreducible of $G$ over $L$. Then the pair $(M, \pi)$ belongs to the cuspidal support if $\Pi$ if there exists a parabolic subgroup $P$ of $G$, with Levi subgroup $M$, such that $\Pi$ is isomorphic to a quotient of $i_{P}^{G} \pi$.

Over a field $L$ of characteristic zero, the notions of cuspidal and supercuspidal support are equivalent, but the notions differ over fields of finite characteristic.

Two pairs $(M, \pi)$ and $\left(M^{\prime}, \pi^{\prime}\right)$ are conjugate in $G$ if there is an element $g$ of $G$ that conjugates $M$ to $M^{\prime}$ and $\pi$ to $\pi^{\prime}$. This determines an equivalence relation on the set of pairs $(M, \pi)$. It is then well known that the cuspidal and supercuspidal support of a given representation $\Pi$ are given by a single conjugacy class of pairs. For cuspidal support, this is an easy consequence of Frobenius reciprocity, but for supercuspidal support this is a deep theorem, first proven by Vigneras [V2, V.4].

DEFINITION 3.3. We say that two representations $\pi, \pi^{\prime}$ of $G$ that differ by a twist by $\chi \circ$ det, where $\chi$ is an unramified character of $F^{\times}$, are inertially equivalent. More generally, if $M$ is a Levi subgroup of $G$, two representations $\pi$ and $\pi^{\prime}$ are inertially equivalent if they differ by a twist by an unramified character $\chi$ of $M$, that is, a character $\chi$ trivial on all compact open subgroups of $M$. 
We are primarily interested in cuspidal and supercuspidal support up to inertial equivalence. Two pairs $(M, \pi)$ and $\left(M^{\prime}, \pi^{\prime}\right)$ are inertially equivalent if there is a representation $\pi^{\prime \prime}$ of $M$, inertially equivalent to $\pi$, such that $\left(M, \pi^{\prime \prime}\right)$ is conjugate to $\left(M^{\prime}, \pi^{\prime}\right)$. The inertial supercuspidal support (respectively inertial cuspidal support) of an absolutely irreducible representation $\Pi$ of $G$ is the inertial equivalence class of its supercuspidal support (respectively cuspidal support).

Theorem 3.4 (Bernstein-Deligne, [BD, 2.13]). Let $M$ be a Levi subgroup of $G$, and let $\pi$ be an irreducible cuspidal representation of $M$. Let $\operatorname{Rep}_{\overline{\mathcal{K}}}(G)_{M, \pi}$ be the full subcategory of $\operatorname{Rep}_{\overline{\mathcal{K}}}(G)$ consisting of representations $\Pi$ such that every simple subquotient of $\Pi$ has inertial supercuspidal support $(M, \pi)$. Then $\operatorname{Rep}_{\overline{\mathcal{K}}}(G)_{M, \pi}$ is a direct factor of $\operatorname{Rep}_{\overline{\mathcal{K}}}(G)$.

There is thus an idempotent $e_{M, \pi, \overline{\mathcal{K}}}$ of the Bernstein center of $\operatorname{Rep}_{\overline{\mathcal{K}}}(G)$ that acts by the identity on all objects of $\operatorname{Rep}_{\overline{\mathcal{K}}}(G)_{M, \pi}$ and annihilates all of the other Bernstein components.

Moreover, it is possible to give a complete description of the center $A_{M, \pi}$ of $\operatorname{Rep}_{\overline{\mathcal{K}}}(G)_{M, \pi}$. Let $\Psi(M)$ denote the group of unramified characters of $M$. Then $\Psi(M)$ can be identified with the algebraic torus $\operatorname{Spec} \overline{\mathcal{K}}\left[M / M_{0}\right]$, where $M_{0}$ is the subgroup of $M$ generated by all compact open subgroups of $M$. The group $\Psi(M)$ acts transitively (by twisting) on the space of representations of $M$ inertially equivalent to $\pi$, and the stabilizer of $\pi$ is a finite subgroup $H$ of $\Psi(M)$. Note that $H$ depends only on the inertial equivalence class of $\pi$, not $\pi$ itself. The group $\Psi(M) / H$ is a torus, isomorphic to $\operatorname{Spec} \overline{\mathcal{K}}\left[M / M_{0}\right]^{H}$; a choice of $\pi$ identifies $\Psi(M) / H$ with the space of representations of $M$ inertially equivalent to $\pi$.

Let $W_{M}$ be the subgroup of the Weyl group $W(G)$ of $G$ (taken with respect to a maximal torus contained in $M$ ) consisting of elements $w$ of $W(G)$ such that $w M w^{-1}=M$. Define a subgroup $W_{M}(\pi)$ of $W_{M}$ consisting of all $w$ in $W_{M}$ such that $\pi^{w}$ is inertially equivalent to $\pi$ (this subgroup depends only on the inertial equivalence class of $\pi$ ). Then $W_{M}(\pi)$ acts on the space of representations of $M$ inertially equivalent to $\pi$, and hence (via a choice of $\pi$ ) on the torus $\operatorname{Spec} \overline{\mathcal{K}}\left[M / M_{0}\right]^{H}$. This action is in general a twist of the usual (permutation) action of $W_{M}$ on $M / M_{0}$, but if $\pi$ is invariant under the action of $W_{M}(\pi)$, then the action of $W_{M}(\pi)$ on $M / M_{0}$ is untwisted.

We have:

THEOREM 3.5 (Bernstein-Deligne). A choice of $\pi$ yields an identification of $A_{M, \pi}$ with the ring $\left(\overline{\mathcal{K}}\left[M / M_{0}\right]^{H}\right)^{W_{M}(\pi)}$. More canonically, the space of representations of $M$ inertially equivalent to $\pi$ is naturally a $\Psi / H$-torsor with an action of $W_{M}(\pi)$, and the center of $\operatorname{Rep}_{\overline{\mathcal{K}}}(G)$ is the ring of $W_{M}(\pi)$-invariant 
regular functions on this torsor. Moreover, if $f$ is an element of $A_{M, \pi}$, and $\Pi$ is an object of $\operatorname{Rep}_{\overline{\mathcal{K}}}(G)_{M, \pi}$ with supercuspidal support $\left(M, \pi^{\prime}\right)$, then $f$ acts on $\Pi$ by the scalar $f\left(\pi^{\prime}\right)$.

We conclude with a standard result describing the action of the Bernstein center on modules arising by parabolic induction. Let $\left(M_{i}, \pi_{i}\right)$ be pairs consisting of a Levi subgroup $M_{i}$ of $\mathrm{GL}_{n_{i}}(F)$, and an irreducible cuspidal representation $\pi_{i}$ of $M_{i}$ such that $\pi_{i}$ is invariant under the action of $W_{M_{i}}\left(\pi_{i}\right)$. Let $M$ be the product of the $M_{i}$, considered as a subgroup of $\mathrm{GL}_{n}(F)$, where $n$ is the sum of the $n_{i}$. Let $\pi$ be the tensor product of the $\pi_{i}$; it is an irreducible cuspidal representation of $M$. We then have an action of $W_{M}(\pi)$ on the inertial equivalence class of $(M, \pi)$.

In this setting, the group $\left(M / M_{0}\right)^{H}$ is the product of the groups $\left(M_{i} /\left(M_{i}\right)_{0}\right)^{H_{i}}$, where $H_{i}$ is the subgroup of characters fixing $\pi_{i}$ under twist. The isomorphism:

$$
\overline{\mathcal{K}}\left[M / M_{0}\right]^{H} \cong \bigotimes_{i} \overline{\mathcal{K}}\left[M_{i} /\left(M_{i}\right)_{0}\right]^{H_{i}}
$$

then restricts to give an embedding:

$$
\Phi:\left(\overline{\mathcal{K}}\left[M / M_{0}\right]^{H}\right)^{W_{M}(\pi)} \hookrightarrow \bigotimes_{i}\left(\overline{\mathcal{K}}\left[M_{i} /\left(M_{i}\right)_{0}\right]^{H_{i}}\right)^{W_{M_{i}}\left(\pi_{i}\right)} .
$$

PROPOSITION 3.6. Let $\Pi_{i}$ be a collection of representations of $\mathrm{GL}_{n_{i}}$ such that for each $i, \Pi_{i}$ lies in $\operatorname{Rep}_{\overline{\mathcal{K}}}\left(\operatorname{GL}_{n_{i}}(F)\right)_{M_{i}, \pi_{i}}$. Let $P=L U$ be a parabolic subgroup of $\mathrm{GL}_{n}(F)$, with $L$ isomorphic to the product of the $\mathrm{GL}_{n_{i}}(F)$, and let $\Pi$ be the tensor product of the $\Pi_{i}$, considered as a representation of $L$. Then $i_{P}^{\mathrm{GL}_{n}(F)} \Pi$ lies in $\operatorname{Rep}_{\overline{\mathcal{K}}}\left(\mathrm{GL}_{n}(F)\right)_{M, \pi}$.

Moreover, under the identifications

$$
\begin{gathered}
A_{M, \pi} \cong\left(\overline{\mathcal{K}}\left[M / M_{0}\right]^{H}\right)^{W_{M}(\pi)}, \\
A_{M_{i}, \pi_{i}} \cong\left(\overline{\mathcal{K}}\left[M_{i} /\left(M_{i}\right)_{0}\right]^{H_{i}}\right)^{W_{M_{i}}\left(\pi_{i}\right)}
\end{gathered}
$$

induced by $(M, \pi)$ and $\left(M_{i}, \pi_{i}\right)$, if $x$ lies in $A_{M, \pi}$, then the endomorphism of $i_{P}^{\mathrm{GL}_{n}(F)} \Pi$ induced by $x$ coincides with the endomorphism of $i_{P}^{\mathrm{GL}_{n}(F)} \Pi$ arising from the action of $\Phi(x)$ on $\Pi$.

\section{Construction of projectives}

Our goal is to apply the theory of Section 2 to the category $\operatorname{Rep}_{W(k)}(G)$ of smooth $W(k)[G]$-modules. In particular, we will factor this category as a product of blocks, and construct an explicit faithfully projective module in each block. The first step is to obtain a supply of suitable projective $W(k)[G]$-modules, and study their properties. 
DEFINITION 4.1. Let $R$ be a $W(k)$-algebra. By an $R$-type of $G$, we mean a pair ( $K, \tau)$, where $K$ is a compact open subgroup of $G$ and $\tau$ is an $R[K]$-module that is finitely generated as an $R$-module. The Hecke algebra $H(G, K, \tau)$ is the ring $\operatorname{End}_{R[G]}\left(\mathrm{c}-\operatorname{Ind}_{K}^{G} \tau\right)$.

For the most part we will be concerned with $R$-types for $R=k$, or $R=\mathcal{K}$, where $\mathcal{K}$ is the fraction field of $W(k)$. Note that for any $R[G]$-module $\pi$, Frobenius reciprocity identifies $\operatorname{Hom}_{R[K]}(\tau, \pi)$ with $\operatorname{Hom}_{R[G]}\left(\mathrm{c}-\operatorname{Ind}_{K}^{G} \tau, \pi\right)$, and hence gives an action of $H(G, K, \tau)$ on $\operatorname{Hom}_{R[K]}(\tau, \pi)$. Moreover, if $V$ is the underlying $R$-module of $\tau, H(G, K, \tau)$ can be identified with the convolution algebra of compactly supported smooth functions $f: G \rightarrow \operatorname{End}_{R}(V)$ that are left and right $K$-invariant, in the sense that $f\left(k g k^{\prime}\right)=\tau(k) f(g) \tau\left(k^{\prime}\right)$. If $g$ is in $G$, then we denote by $I_{g}(\tau)$ the space $\operatorname{Hom}_{K \cap g K g^{-1}}\left(\tau, \tau^{g}\right)$, where $\tau^{g}$ is the representation of $g K^{-1}$ defined by $\tau^{g}(k)=\tau\left(g^{-1} k g\right)$. Then the map $f \mapsto f(g)$ is an isomorphism:

$$
H(G, K, \tau)_{K g K} \cong I_{g}(\tau),
$$

where $H(G, K, \tau)_{K g K}$ is the space of functions in $H(G, K, \tau)$ supported on $K g K$.

In this section, we will primarily be concerned with a certain class of types which are called maximal distinguished cuspidal types in [V2, IV.3.1B]. We omit the precise definition of these types here; for our purposes it suffices to know certain specific properties of a maximal distinguished cuspidal $R$-type $(K, \tau)$, where $R$ is a field.

Such a type arises from a simple stratum $[\mathfrak{A}, n, 0, \beta]$, together with a character $\theta$ in the set $\mathcal{C}(\mathfrak{A}, 0, \beta)$ defined in [BK1, 3.2.1]. Here $\mathfrak{A}$ is a maximal order in $M_{n}(F)$, and $\beta$ is an element of $\mathfrak{A}$ such that $E=F[\beta]$ is a field. This allows us to identify $E^{\times}$with a subgroup of $\operatorname{GL}_{n}(F)$. Let $e$ and $f$ denote the ramification index and residue class degree of $E$ over $F$. One then has:

- $K$ is the group $J(\beta, \mathfrak{A})$ of [BK1]. In particular, $K$ contains a normal pro$p$ subgroup $K^{1}$ (called $J^{1}(\beta, \mathfrak{A})$ in [BK1]), such that the quotient $K / K^{1}$ is isomorphic to $\mathrm{GL}_{n / e f}\left(\mathbb{F}_{q^{f}}\right)$, where $q$ is the order of the residue field of $F$.

- $\tau$ has the form $\kappa \otimes \sigma$, where $\sigma$ is the inflation of a cuspidal representation of $K / K^{1}$ over $R$, and $\kappa$ is a representation of $K$ that is a $\beta$-extension of the unique irreducible representation of $K^{1}$ containing $\theta$.

Maximal distinguished cuspidal $R$-types have the following useful properties:

THEOREM 4.2 [V2, IV.1.1-IV.1.3]. Let $R$ be a field, and let $(K, \tau)$ be a maximal distinguished cuspidal $R$-type arising from an extension $E / F$. 
(1) There is a unique embedding of $\mathrm{GL}_{n / e f}(E)$ into $G$ such that the center $E^{\times}$ of $\mathrm{GL}_{n / e f}(E)$ normalizes $K$ and $K^{1}$, and acts trivially on $K / K^{1}$. We identify $\mathrm{GL}_{n / e f}(E)$ and $E^{\times}$with their images under this embedding. The intersection of $\mathrm{GL}_{n / e f}(E)$ with $K$ is $\mathrm{GL}_{n / e f}\left(\mathcal{O}_{E}\right)$.

(2) The subgroup $E^{\times}$of $G$ normalizes $\tau$. In Particular, $\tau$ extends to a representation of $E^{\times} K$, and any two extensions of $\tau$ differ by a twist by a character of $E^{\times} K / K \cong \mathbb{Z}$.

(3) The G-intertwining of $(K, \tau)$ is equal to $E^{\times} K$.

(4) For any extension $\hat{\tau}$ of $\tau$ to a representation of $E^{\times} K$, there is an isomorphism of $H(G, K, \tau)$ with the polynomial ring $R\left[T, T^{-1}\right]$, that sends $T$ to the unique element $f_{\hat{\tau}}$ of $H(G, K, \tau)$ such that $f_{\hat{\tau}}$ is supported on $K \varpi_{E} K$ (where $\varpi_{E}$ is a uniformizer of $E$ ), and

$$
f_{\hat{\tau}}\left(k \varpi_{E} k^{\prime}\right)=\tau(k) \hat{\tau}\left(\varpi_{E}\right) \tau\left(k^{\prime}\right) .
$$

(5) For any extension $\hat{\tau}$ of $\tau$ to a representation of $E^{\times} K$, the representation c-Ind ${ }_{E^{\times} K}^{G} \hat{\tau}$ is an irreducible cuspidal representation of $G$ over $R$.

(6) Every irreducible cuspidal representation of $G$ over $R$ arises in this fashion. Those irreducible cuspidal $\pi$ that arise from a given $(K, \tau)$ are precisely those $\pi$ whose restriction to $K$ contains $\tau$.

If $R$ is a field, and $\pi, \pi^{\prime}$ are absolutely irreducible cuspidal $R$-representations containing a maximal distinguished cuspidal type $(K, \tau)$, then $\operatorname{Hom}_{R[K]}(\tau, \pi)$ and $\operatorname{Hom}_{R[K]}\left(\tau, \pi^{\prime}\right)$ are modules over $H(G, K, \tau)=R\left[T, T^{-1}\right]$ that are onedimensional as $R$-vector spaces. In particular, $T$ acts via scalars $c$ and $c^{\prime}$ on $\operatorname{Hom}_{R[K]}(\tau, \pi)$ and $\operatorname{Hom}_{R[K]}\left(\tau, \pi^{\prime}\right)$, respectively. Let $\chi$ be an unramified $k$-valued character of $F^{\times}$such that $\chi\left(\varpi_{F}\right)^{n / e}=c^{\prime} c^{-1}$. As $T$ is supported on $K \varpi_{E} K$, and det $\varpi_{E}=\varpi_{F}^{n / e}$, the $H(G, K, \tau)$-modules $\operatorname{Hom}_{R[K]}(\tau, \pi \otimes \chi \circ \operatorname{det})$ and $\operatorname{Hom}_{R[K]}\left(\tau, \pi^{\prime}\right)$ are isomorphic, and so $\pi^{\prime}$ is a twist of $\pi$ by an unramified character; that is, $\pi$ and $\pi^{\prime}$ are inertially equivalent.

REMARK 4.3. The isomorphism of $R\left[T, T^{-1}\right]$ with $H(G, K, \tau)$ depends on a choice of extension $\hat{\tau}$ of $\tau$, and also a uniformizer $\varpi_{E}$. When $R$ is a field, this isomorphism may be reinterpreted in the language Bernstein and Deligne use to describe the Bernstein center, and made independent of $\varpi_{E}$ (but not of $\hat{\tau}$ ). Let $\pi$ be the irreducible cuspidal representation of $G$ whose restriction to $E^{\times} K$ contains $\hat{\tau}$. Then every representation of $G$ inertially equivalent to $\pi$ has the form $\pi \otimes \chi$ for some unramified character $\chi$ of $G / G_{0}$, and we have $\pi=\pi \otimes \chi$ if, and only if, 
$\pi \otimes \chi$ contains $\hat{\tau}$. The latter holds precisely when $\hat{\tau}=\left.\hat{\tau} \otimes \chi\right|_{E^{\times} K}$, which holds if and only if $\chi\left(\varpi_{E}\right)=1$. Let $Z$ be the subgroup of $G$ generated by $\varpi_{E}$; our choice of $\varpi_{E}$ identifies $R\left[T, T^{-1}\right]$ with $R[Z]$. The map $Z \rightarrow G / G_{0}$ is injective (but not in general surjective), and induces a map

$$
\operatorname{Hom}\left(G / G_{0}, \mathbb{G}_{m}\right) \rightarrow \operatorname{Hom}\left(Z, \mathbb{G}_{m}\right)
$$

by restriction. Let $H$ be the kernel of this map; the induced map on rings of regular functions then identifies $R[Z]$ with the $H$-invariants $R\left[G / G_{0}\right]^{H}$. The identifications:

$$
H(G, K, \tau) \cong R\left[T, T^{-1}\right] \cong R[Z] \cong R\left[G / G_{0}\right]^{H}
$$

give an identification of $H(G, K, \tau)$ with $R\left[G / G_{0}\right]^{H}$ that does not depend on $\varpi_{E}$. The map $\chi \mapsto \pi \otimes \chi$ describes a bijection between $\left(\operatorname{Spec} R\left[G / G_{0}\right]\right) / H$ and the set of representations of $G$ inertially equivalent to $\pi$. Under the above isomorphisms, the character of $H(G, K, \tau)$ that corresponds to a representation $\pi \otimes \chi$ of $G$ is the character of $R\left[G / G_{0}\right]^{H}$ obtained by treating $R\left[G / G_{0}\right]$ as the ring of regular functions on the space of unramified characters of $G$ and evaluating such functions at $\chi$.

Let $\mathcal{K}^{\prime}$ be a finite extension of the field of fractions $\mathcal{K}$ of $W(k)$, and let $\mathcal{O}$ be its ring of integers. If $\pi$ is an absolutely irreducible cuspidal integral representation of $G$ over $\mathcal{K}^{\prime}$, then $\pi$ contains a unique homothety class of $G$-stable $\mathcal{O}$-lattices, and the reduction $r_{\ell} \pi$ of any such lattice modulo $\ell$ is an absolutely irreducible cuspidal representation of $G$ over $k$. In this situation we have the following compatibilities between the types attached to $\pi$ and $r_{\ell} \pi$, due to Vigneras:

THEOREM 4.4 [V2, IV.1.5]. Let $\pi$ be an irreducible cuspidal representation of $G$ over $\mathcal{K}^{\prime}$, containing a maximal distinguished cuspidal $\mathcal{K}^{\prime}$-type $(K, \tilde{\tau})$.

(1) There is an unramified character $\chi: F^{\times} \rightarrow\left(\mathcal{K}^{\prime}\right)^{\times}$, such that $\pi \otimes(\chi \circ$ det $)$ is integral.

(2) The $K$-representation $\tilde{\tau}$ is defined over $\mathcal{K}$, and the mod $\ell$ reduction of $\tilde{\tau}$ is an irreducible $k$-representation $\tau$ of $K$, such that $(K, \tau)$ is a maximal distinguished cuspidal $k$-type contained in $r_{\ell}[\pi \otimes(\chi \circ$ det $)]$.

(3) Every maximal distinguished cuspidal $k$-type arises from a maximal distinguished cuspidal $\mathcal{K}$ '-type via 'reduction mod $\ell$ ', for some finite extension $\mathcal{K}^{\prime}$ of $\mathcal{K}$. 
We can use this theory of reduction $\bmod \ell$ to turn inertial equivalence into an equivalence relation on simple cuspidal smooth $W(k)[G]$-modules $\pi$. Such $\pi$ fall into two classes: either $\ell$ annihilates $\pi$, in which case $\pi$ is an irreducible cuspidal $k$-representation of $G$, or $\ell$ is invertible on $\pi$, in which case $\pi$ is an absolutely irreducible cuspidal representation of $G$ over some finite extension $\mathcal{K}^{\prime}$ of $\mathcal{K}$.

LEMMA 4.5. Let $\pi$ be a simple smooth $W(k)[G]$-module on which $\ell$ is invertible, and let $\mathcal{K}^{\prime}$ be a finite extension of $\mathcal{K}$ such that every $\mathcal{K}^{\prime}[G]$-simple subquotient of $\pi \otimes_{\mathcal{K}} \mathcal{K}^{\prime}$ is absolutely simple. Then $\pi \otimes_{\mathcal{K}} \mathcal{K}^{\prime}$ is a direct sum of absolutely simple $\mathcal{K}^{\prime}[G]$-modules, and $\operatorname{Gal}(\overline{\mathcal{K}} / \mathcal{K})$ acts transitively on these summands.

Proof. Let $\pi_{0}$ be an absolutely simple $\mathcal{K}^{\prime}[G]$-submodule of $\pi \otimes_{\mathcal{K}} \mathcal{K}^{\prime}$. Then the sum of the submodules $\pi_{0}^{g}$ for $g$ in $\operatorname{Gal}(\overline{\mathcal{K}} / \mathcal{K})$ is a Galois-stable $\mathcal{K}^{\prime}[G]$-submodule of $\pi \otimes_{\mathcal{K}} \mathcal{K}^{\prime}$, and hence descends to a $\mathcal{K}[G]$-submodule of $\pi$. This submodule must be all of $\pi$, and the result follows.

DEFINITION 4.6. Let $(K, \tau)$ be a maximal distinguished cuspidal $k$-type, and let $\pi$ be a simple cuspidal smooth $W(k)[G]$-module. We say that $\pi$ belongs to the mod $\ell$ inertial equivalence class determined by $(K, \tau)$ if either $\ell$ annihilates $\pi$ and $\pi$ contains $(K, \tau)$, or if $\ell$ is invertible on $\pi$ and there exists a finite extension $\mathcal{K}^{\prime}$ of $\mathcal{K}$ such that one (equivalently, every) absolutely simple summand of $\pi \otimes_{\mathcal{K}} \mathcal{K}^{\prime}$ is inertially equivalent to an integral representation of $G$ over $\mathcal{K}^{\prime}$ whose $\bmod \ell$ reduction contains $(K, \tau)$.

Fix a maximal distinguished cuspidal $k$-type $(K, \tau)$, with $\tau=\kappa \otimes \sigma$, and let $\mathcal{P}_{\sigma} \rightarrow \sigma$ be the projective envelope of $\sigma$ in the category of $W(k)\left[G L_{n / e f}\left(\mathbb{F}_{q^{f}}\right)\right]$ modules. We then have:

LEMMA 4.7. The representation $\kappa$ lifts to a representation $\tilde{\kappa}$ of $K$ over $W(k)$.

Proof. As $K_{1}$ is a pro-p-group, the restriction $\kappa_{1}$ of $\kappa$ to $K_{1}$ lifts uniquely to a representation $\tilde{\kappa}_{1}$ of $K_{1}$ over $W(k)$, normalized by $K$. The obstruction to extending $\tilde{\kappa}_{1}$ to $K$ is thus an element of $H^{2}\left(K, W(k)^{\times}\right)$. The first two paragraphs of [BK1], Proposition 5.2.4, show that this element can be represented by a cocycle taking values in the $p$-power roots of unity, and is thus a $p$-power torsion element $\alpha$ of $H^{2}\left(K, W(k)^{\times}\right)$. (In [BK1] the authors work over $\mathbb{C}$ rather than $W(k)$, but their argument adapts without difficulty. Note that they denote by $\eta_{M}$ the representation we call $\tilde{\kappa}_{1}$, by $J_{M}$ the group we call $K$, and $J_{M}^{1}$ the group we call $K_{1}$.)

Let $U$ be a $p$-sylow subgroup of $K$ containing $K_{1}$. The restriction of $\kappa$ to $U$ lifts uniquely to a representation over $W(k)$, extending $\tilde{\kappa}_{1}$. It follows that the image of 
$\alpha$ in $H^{2}\left(U, W(k)^{\times}\right)$under restriction vanishes. But the corestriction of this image to $H^{2}\left(K, W(k)^{\times}\right)$is equal to $r \alpha$, where $r$ is the index of $U$ in $K$. Thus $\alpha$ is killed by a power of $p$ and an integer prime to $p$, and must therefore vanish.

LEMMA 4.8. The tensor product $\tilde{\kappa} \otimes \mathcal{P}_{\sigma}$ is a projective envelope of $\kappa \otimes \sigma$ in the category of $W(k)[K]$-modules.

Proof. The restriction of $\kappa$ to $K_{1}$ is irreducible, and the restriction of $\tilde{\kappa} \otimes \mathcal{P}_{\sigma}$ to $K_{1}$ is a direct sum of copies of $\tilde{\kappa}_{1}$. We thus have isomorphisms of $W(k)\left[K / K_{1}\right]$ modules:

$$
\operatorname{Hom}_{K_{1}}\left(\tilde{\kappa}, \tilde{\kappa} \otimes \mathcal{P}_{\sigma}\right) \cong \mathcal{P}_{\sigma} .
$$

(Here $g \in K / K_{1}$ acts on $\operatorname{Hom}_{K_{1}}\left(\tilde{\kappa}, \tilde{\kappa} \otimes \mathcal{P}_{\sigma}\right)$ by $f \mapsto f^{g}$, where $f^{g}(x)=$ $g f\left(g^{-1} x\right)$; note that this action depends on $\tilde{\kappa}$, not just its restriction to $K_{1}$.)

Now suppose we have a surjection:

$$
\theta^{\prime} \rightarrow \theta
$$

of $W(k)[K]$-modules. We need to show that any map $\tilde{\kappa} \otimes \mathcal{P}_{\sigma} \rightarrow \theta$ lifts to a map to $\theta^{\prime}$. As we have identified $\mathcal{P}_{\sigma}$ with $\operatorname{Hom}_{K_{1}}\left(\tilde{\kappa}, \tilde{\kappa} \otimes \mathcal{P}_{\sigma}\right)$, such a map induces a map $\mathcal{P}_{\sigma} \rightarrow \operatorname{Hom}_{K_{1}}(\tilde{\kappa}, \theta)$. This latter map is $K / K_{1}$-equivariant.

As $K_{1}$ is a pro- $p$ group, the surjection of $\theta^{\prime} \rightarrow \theta$ induces a surjection

$$
\operatorname{Hom}_{K_{1}}\left(\tilde{\kappa}, \theta^{\prime}\right) \rightarrow \operatorname{Hom}_{K_{1}}(\tilde{\kappa}, \theta)
$$

of $W(k)\left[K / K_{1}\right]$-modules. As $\mathcal{P}_{\sigma}$ is projective, the map

$$
\mathcal{P}_{\sigma} \rightarrow \operatorname{Hom}_{K_{1}}(\tilde{\kappa}, \theta)
$$

lifts to a map

$$
\mathcal{P}_{\sigma} \rightarrow \operatorname{Hom}_{K_{1}}\left(\tilde{\kappa}, \theta^{\prime}\right) .
$$

Tensoring with $\tilde{\kappa}$, we obtain the desired map $\tilde{\kappa} \otimes \mathcal{P}_{\sigma} \rightarrow \theta^{\prime}$, so $\tilde{\kappa} \otimes \mathcal{P}_{\sigma}$ is projective.

On the other hand, $\tilde{\kappa} \otimes \mathcal{P}_{\sigma}$ is indecomposable over $W(k)[K]$, and is therefore a projective envelope of $\kappa \otimes \sigma$ in the category of $W(k)[K]$-modules.

As the functor c-Ind ${ }_{K}^{G}$ is a left adjoint of an exact functor, it takes projectives to projectives. In particular, the module $\mathcal{P}_{K, \tau}$ defined by $\mathcal{P}_{K, \tau}:=\mathrm{c}-\operatorname{Ind}_{K}^{G} \tilde{\kappa} \otimes \mathcal{P}_{\sigma}$ is a projective object in $\operatorname{Rep}_{W(k)}(G)$.

PROPOSITION 4.9. Let $\pi$ be a simple cuspidal smooth $W(k)[G]$-module in the mod $\ell$ inertial equivalence class determined by $(K, \tau)$. Then there exists a surjection $\mathcal{P}_{K, \tau} \rightarrow \pi$. 
Proof. First suppose that $\ell$ annihilates $\pi$. The surjection of $\mathcal{P}_{\sigma}$ onto $\sigma$ gives rise to a surjection $\mathcal{P}_{K, \tau} \rightarrow \mathrm{c}-\operatorname{Ind}_{K}^{G} \tau$. As the restriction of $\pi$ to $K$ contains $\tau$, we have a nonzero (thus surjective) map c-Ind $\operatorname{In}_{K}^{G} \tau \rightarrow \pi$ as claimed.

On the other hand, if $\ell$ is invertible in $\pi$, then fix an absolutely simple summand $\pi_{0}$ of $\pi \otimes_{\mathcal{K}} \mathcal{K}^{\prime}$ for some finite extension $\mathcal{K}^{\prime}$ of $\mathcal{K}$. As $\pi_{0}$ is in the $\bmod \ell$ inertial equivalence class determined by $(K, \tau)$, there is a maximal distinguished cuspidal $\mathcal{K}^{\prime}$-type $(K, \tilde{\tau})$ contained in $\pi_{0}$; its $\bmod \ell$ reduction is $(K, \tau)$. If we regard $\tilde{\tau}$ as a representation of $K$ over the ring of integers $\mathcal{O}^{\prime}$ of $\mathcal{K}^{\prime}$, we have surjections

$$
\begin{gathered}
\tilde{\tau} \rightarrow \tau \\
\tilde{\kappa} \otimes \mathcal{P}_{\sigma} \rightarrow \tau,
\end{gathered}
$$

and thus obtain a map $\tilde{\kappa} \otimes \mathcal{P}_{\sigma} \rightarrow \tilde{\tau}$ by projectivity of $\tilde{\kappa} \otimes \mathcal{P}_{\sigma}$. This map is necessarily surjective, so by applying the functor $\mathrm{c}-\operatorname{Ind}_{K}^{G}$, we obtain a surjection

$$
\mathcal{P}_{K, \tau} \otimes_{W(k)} \mathcal{O}^{\prime} \rightarrow \mathrm{c}-\operatorname{Ind}_{K}^{G} \tilde{\tau}
$$

Composing this surjection with the nonzero maps

$$
\mathrm{c}-\operatorname{Ind}_{K}^{G} \tilde{\tau} \rightarrow \mathrm{c}-\operatorname{Ind}_{K}^{G}(\tilde{\tau}) \otimes_{W(k)} \mathcal{K} \rightarrow \pi_{0}
$$

yields a nonzero map $\mathcal{P}_{K, \tau} \otimes_{\mathcal{K}} \mathcal{K}^{\prime} \rightarrow \pi_{0}$, and hence a nonzero map

$$
\mathcal{P}_{K, \tau} \otimes_{\mathcal{K}} \mathcal{K}^{\prime} \rightarrow \pi \otimes_{\mathcal{K}} \mathcal{K}^{\prime}
$$

As $\operatorname{Hom}_{\mathcal{K}^{\prime}[G]}\left(\mathcal{P}_{K, \tau} \otimes_{\mathcal{K}} \mathcal{K}^{\prime}, \pi \otimes_{\mathcal{K}} \mathcal{K}^{\prime}\right)$ is isomorphic to $\operatorname{Hom}_{\mathcal{K}[G]}\left(\mathcal{P}_{K, \tau}, \pi\right) \otimes \mathcal{K}^{\prime}$, there exists a nonzero map from $\mathcal{P}_{K, \tau}$ to $\pi$, which must be surjective by simplicity of $\pi$.

It will follow from results in Section 8 that not every simple quotient of $\mathcal{P}_{K, \tau}$ has the above form.

Our next goal is to use the $\mathcal{P}_{K, \tau}$ to construct projectives that admit surjections onto representations with given cuspidal support. We must first introduce some additional language. As a maximal distinguished cuspidal $k$-type determines an inertial equivalence class of cuspidal representations, we will sometimes say that the supercuspidal or cuspidal support of a representation $\Pi$ is given by a collection $\left\{\left(K_{1}, \tau_{1}\right), \ldots,\left(K_{r}, \tau_{r}\right)\right\}$ of maximal distinguished cuspidal $k$-types; this means that $\Pi$ has supercuspidal (or cuspidal) support $(M, \pi)$, where $M$ is a 'block diagonal' subgroup of the form $\mathrm{GL}_{n_{1}} \times \cdots \times \mathrm{GL}_{n_{r}}$, and $\pi$ is a tensor product $\pi_{1} \otimes \cdots \otimes \pi_{r}$ where $\pi_{i}$ is in the inertial equivalence class determined by $\left(K_{i}, \tau_{i}\right)$ for all $i$. 
DEFINITION 4.10. Let $M$ be a Levi subgroup of $G$ and let $\pi$ be an irreducible cuspidal representation of $M$ over $k$. Let $\Pi$ be a simple smooth $W(k)$-module. We say that $(M, \pi)$ belongs to the mod $\ell$ inertial cuspidal support of $\Pi$ if either:

(1) $\Pi$ is killed by $\ell$, and its cuspidal support is the inertial equivalence class of $(M, \pi)$; or

(2) $\ell$ is invertible on $\Pi$, and there exists a finite extension $\mathcal{K}^{\prime}$ of $\mathcal{K}$, with ring of integers $\mathcal{O}^{\prime}$, such that $\Pi \otimes_{\mathcal{K}} \mathcal{K}^{\prime}$ is a direct sum of absolutely simple $\mathcal{K}^{\prime}[G]$ modules, and for some (equivalently every) absolutely simple summand $\Pi_{0}$ of $\Pi \otimes_{\mathcal{K}} \mathcal{K}^{\prime}$, there exists a smooth $\mathcal{O}^{\prime}$-integral representation $\tilde{\pi}$ of $M$ lifting $\pi$, such that the cuspidal support of $\Pi_{0}$ is the inertial equivalence class of $(M, \tilde{\pi})$.

In this language, Proposition 4.9 says that every simple $W(k)[G]$-module with $\bmod \ell$ inertial cuspidal support given by $(K, \tau)$ is a quotient of $\mathcal{P}_{K, \tau}$.

We will also need a notion of $\bmod \ell$ inertial supercuspidal support. We first recall a standard result about the behavior of supercuspidal support under reduction $\bmod \ell$ :

Proposition 4.11 [V3, 1.5]. Let $\tilde{\Pi}$ be an absolutely irreducible smooth integral representation of $G$ over a finite extension $\mathcal{K}^{\prime}$ of $\mathcal{K}$, with supercuspidal support $(M, \tilde{\pi})$. Then $\tilde{\pi}$ is an integral representation of $M$. Moreover, let $\Pi$ and $\pi$ denote the mod $\ell$ reductions of $\tilde{\Pi}$ and $\tilde{\pi}$, respectively. Then $\pi$ is irreducible and cuspidal (but not necessarily supercuspidal). Moreover, the supercuspidal support of any simple subquotient of $\Pi$ is equal to the supercuspidal support of $\pi$.

DEFINITION 4.12. Let $M$ be a Levi subgroup of $G$ and let $\pi$ be an irreducible supercuspidal representation of $M$ over $k$. Let $\Pi$ be a simple smooth $W(k)[G]-$ module. We say that $(M, \pi)$ belongs to the mod $\ell$ inertial supercuspidal support of $\Pi$ if there exists a Levi subgroup $M^{\prime}$ of $G$ containing $M$, and an irreducible cuspidal representation $\pi^{\prime}$ of $M^{\prime}$ over $k$, such that $\Pi$ has $\bmod \ell$ inertial cuspidal support containing $\left(M^{\prime}, \pi^{\prime}\right)$, and $\pi^{\prime}$ has supercuspidal support containing $(M, \pi)$.

Proposition 4.13. Let $\Pi$ be an irreducible smooth integral representation of $G$ over a finite extension $\mathcal{K}^{\prime}$ of $\mathcal{K}$. The following are equivalent:

(1) $\Pi$ has mod $\ell$ inertial supercuspidal support $(M, \pi)$.

(2) Every simple subquotient of the mod $\ell$ reduction of $\Pi$ has supercuspidal support inertially equivalent to $(M, \pi)$. 
Proof. This is immediate from Proposition 4.11.

If $M$ is a Levi subgroup of $G$, and $\pi$ is an irreducible cuspidal representation of $M$ over $k$, define $\mathcal{P}_{(M, \pi)}$ to be the normalized parabolic induction

$$
\mathcal{P}_{(M, \pi)}:=i_{P}^{G}\left[\mathcal{P}_{K_{1}, \tau_{1}} \otimes \cdots \otimes \mathcal{P}_{K_{r}, \tau_{r}}\right],
$$

where $P$ is a parabolic subgroup whose associate Levi subgroup is $M$, and the $\left(K_{i}, \tau_{i}\right)$, are a sequence of maximal distinguished cuspidal $k$-types whose associated $\bmod \ell$ inertial equivalence class is $(M, \pi)$.

REMARK 4.14. Strictly speaking, $\mathcal{P}_{(M, \pi)}$ may depend on the choice of $P$, as well as the particular pairs $\left(K_{i}, \tau_{i}\right)$; we suppress these dependences from the notation. In fact, it seems likely that changing $P$, or replacing some of the $\left(K_{i}, \tau_{i}\right)$ with equivalent types, give rise to isomorphic modules $\mathcal{P}_{(M, \pi)}$, but we will not need this and do not attempt to prove it.

LEMMA 4.15. Let $\Pi$ be an absolutely irreducible representation of $G$ over $k$ or a finite extension $\mathcal{K}^{\prime}$ of $\mathcal{K}$, and let $\pi_{1}, \ldots, \pi_{r}$ be a sequence of absolutely irreducible cuspidal representations such that $\Pi$ is a quotient of $i_{P}^{G}\left[\pi_{1} \otimes \cdots \otimes \pi_{r}\right]$. Let $s_{i}$ be the permutation of $1, \ldots, r$ that interchanges $i$ and $i+1$ and fixes all other integers. Then either $\Pi$ is a quotient of $i_{P}^{G}\left[\pi_{s_{i}(1)} \otimes \cdots \otimes \pi_{s_{i}(r)}\right]$, or $\pi_{i}$ and $\pi_{i+1}$ are inertially equivalent.

Proof. The parabolic induction $i_{P}^{G}\left[\pi_{1} \otimes \cdots \otimes \pi_{r}\right]$ is isomorphic to

$$
i_{P}^{G}\left[\pi_{s_{i}(1)} \otimes \cdots \otimes \pi_{s_{i}(r)}\right]
$$

unless $\pi_{i}=(|| \circ \mathrm{det})^{ \pm 1} \pi_{i+1}$. If this is the case then $\pi_{i}$ is inertially equivalent to $\pi_{i+1}$.

Proposition 4.16. let $\Pi$ be a simple smooth $W(k)[G]$-module with mod $\ell$ cuspidal support given by the inertial equivalence class $(M, \pi)$. Then $\Pi$ is a quotient of $\mathcal{P}_{(M, \pi)}$.

Proof. Either $\Pi$ is defined and absolutely irreducible over $k$, or $\ell$ is invertible on $\Pi$ and there exists a finite extension $\mathcal{K}^{\prime}$ of $\mathcal{K}$ such that $\Pi \otimes_{\mathcal{K}} \mathcal{K}^{\prime}$ is a direct sum of absolutely simple $\mathcal{K}^{\prime}[G]$-modules. Choose an absolutely irreducible cuspidal representation $\tilde{\pi}$ of $M$, defined over the appropriate field $\left(k\right.$ or $\left.\mathcal{K}^{\prime}\right)$ such that, over this field, $\Pi$ admits a nonzero map from $i_{P}^{G} \pi$. Write $\pi=\pi_{1} \otimes \cdots \otimes \pi_{r}$, and choose $\left(K_{i}, \tau_{i}\right)$ maximal distinguished cuspidal types such that $\pi_{i}$ is in the inertial 
equivalence class determined by $\left(K_{i}, \tau_{i}\right)$ for all $i$. Then, for a suitable parabolic subgroup $P$, we have

$$
\mathcal{P}_{M, \pi}=i_{P}^{G}\left[\mathcal{P}_{\left(K_{1}, \tau_{1}\right)} \otimes \cdots \otimes \mathcal{P}_{\left(K_{r}, \tau_{r}\right)}\right] .
$$

Reorder the $\tilde{\pi}_{i}$ such that for all $i \tilde{\pi}_{i}$ is in the $\bmod \ell$ inertial equivalence class determined by $\left(K_{i}, \tau_{i}\right)$. By the previous lemma, we may do this and still assume that there is a nonzero map from $i_{P}^{G} \tilde{\pi}$ to $\Pi$. Now we have a nonzero map of $\mathcal{P}_{K_{i}, \tau_{i}}$ into $\tilde{\pi}_{i}$ for each $i$, that is surjective if $\tilde{\pi}$ is defined over $k$ and that becomes surjective after inverting $\ell$ if $\tilde{\pi}$ is defined over some $\mathcal{K}^{\prime}$. Hence, after induction, we obtain a nonzero map of $\mathcal{P}_{(M, \pi)}$ to $i_{P}^{G} \tilde{\pi}$ that is surjective if $\Pi$ is defined over $k$, and becomes surjective after tensoring with $\mathcal{K}^{\prime}$ if $\ell$ is invertible on $\Pi$. If $\Pi$ is defined over $k$, then composing this surjection with the surjection of $i_{P}^{G} \tilde{\pi}$ onto $\Pi$ yields a nonzero map of $\mathcal{P}_{(M, \pi)}$ to $\Pi$; this map must be surjective as $\Pi$ is simple. On the other hand, if $\ell$ is invertible on $\Pi$, we have a nonzero map

$$
\mathcal{P}_{(M, \pi)} \otimes_{W(k)} \mathcal{K}^{\prime} \rightarrow \Pi \otimes_{\mathcal{K}} \mathcal{K}^{\prime}
$$

obtained by composing the surjection of $\mathcal{P}_{(M, \pi)} \otimes \mathcal{K}^{\prime}$ onto $i_{P}^{G} \tilde{\pi}$ with the map $i_{P}^{G} \tilde{\pi} \rightarrow \Pi \otimes_{\mathcal{K}} \mathcal{K}^{\prime}$. We thus have a nonzero map of $\mathcal{P}_{(M, \pi)}$ onto $\Pi$, and the result follows.

We will see later that in fact the $W(k)[G]$-modules $\mathcal{P}_{(M, \pi)}$ are projective, and once we have established this they will form the basic building blocks of our theory.

\section{Finite group theory}

Our first step in understanding the projectives $\mathcal{P}_{(M, \pi)}$ and $\mathcal{P}_{K, \tau}$ is to understand the projective envelope $\mathcal{P}_{\sigma}$ of a representation $\sigma$ of $\mathrm{GL}_{n}\left(\mathbb{F}_{q}\right)$. This mostly uses standard facts from the representation theory of $\mathrm{GL}_{n}\left(\mathbb{F}_{q}\right)$ that we now recall. For conciseness, we let $\bar{G}$ denote the group $\mathrm{GL}_{n}\left(\mathbb{F}_{q}\right)$, and fix an $\ell$ prime to $q$.

If $\bar{P}=\overline{M U}$ is a parabolic subgroup of $\bar{G}$, with Levi subgroup $\bar{M}$ and unipotent radical $\bar{U}$, we have parabolic induction and restriction functors:

$$
\begin{aligned}
i \frac{\bar{G}}{P}: \operatorname{Rep}_{W(k)}(\bar{M}) \rightarrow \operatorname{Rep}_{W(k)}(\bar{G}) \\
r_{\bar{G}}^{\bar{P}}: \operatorname{Rep}_{W(k)}(\bar{G}) \rightarrow \operatorname{Rep}_{W(k)}(\bar{M}) .
\end{aligned}
$$

Here $i \frac{\bar{G}}{P}$ takes a $W(k)[\bar{M}]$-module, considers it as a $W(k)[\bar{P}]$-module by letting $\bar{U}$ act trivially, and induces to $\bar{G}$. Its adjoint $r_{\bar{G}} \bar{P}$ (which is both a left and right adjoint in the finite group case) takes the $\bar{U}$-invariants of a $W(k)[\bar{G}]$-module and considers the resulting space as a $W(k)[\bar{M}]$-module. 
Just as in the representation theory of $\mathrm{GL}_{n}$ over a local field, we can then define:

DEFinition 5.1. A $W(k)[\bar{G}]$-module $\pi$ is cuspidal if $r_{\bar{G}}^{\bar{P}} \pi=0$ for all proper parabolics $\bar{P}$ of $\bar{G}$. An irreducible $k[\bar{G}]$ or $\overline{\mathcal{K}}[\bar{G}]$-module $\pi$ is supercuspidal if it does not arise as a subquotient of $i \frac{\bar{G}}{P} \pi^{\prime}$ for any proper parabolic subgroup $\bar{P}=\overline{M U}$ of $\bar{G}$ and any representation $\pi^{\prime}$ of $\bar{M}$ (over $k$ or $\overline{\mathcal{K}}$, as appropriate).

Over $\overline{\mathcal{K}}$, an irreducible representation $\pi$ is cuspidal if and only if it is supercuspidal; over $k$ a supercuspidal representation is cuspidal but the converse need not hold. We also define:

Definition 5.2. Let $\bar{P}=\overline{M U}$ be a parabolic subgroup of $\bar{G}$, and let $\pi^{\prime}$ be an irreducible representation of $\bar{M}$.

(1) An irreducible $k[\bar{G}]$ or $\overline{\mathcal{K}}[\bar{G}]$-module $\pi$ has cuspidal support $\left(\bar{M}, \pi^{\prime}\right)$ if $\pi^{\prime}$ is cuspidal and $\pi$ is a quotient of $i \frac{\bar{G}}{P} \pi^{\prime}$.

(2) An irreducible $k[\bar{G}]$ or $\overline{\mathcal{K}}[\bar{G}]$-module $\pi^{\prime}$ has supercuspidal support $\left(\bar{M}, \pi^{\prime}\right)$ if $\pi^{\prime}$ is supercuspidal and $\pi$ is a subquotient of $i \frac{\bar{G}}{P} \pi^{\prime}$.

The cuspidal and supercuspidal support of an irreducible $\pi$ always exist, and are unique up to $\bar{G}$-conjugacy. Over $\overline{\mathcal{K}}$ the two notions coincide, but this is not true over $k$ because of the existence of cuspidal representations that are not supercuspidal. As with representations of $\mathrm{GL}_{n}(F)$, the uniqueness of cuspidal support (up to $\bar{G}$-conjugacy) is an immediate consequence of Frobenius reciprocity, but the uniqueness of supercuspidal support is deep and requires work (in the setting of general linear groups over finite fields, it follows from the classification of irreducible modular representations of $\bar{G}$ due to James [Ja].

As the parabolic induction and restriction functors are defined on the level of $W(k)[\bar{G}]$-modules, it is clear that the reduction $\bmod \ell$ of a cuspidal representation is cuspidal. The notion of supercuspidal support is compatible with reduction mod $\ell$ in the following sense: if $\tilde{\pi}$ is an irreducible representation of $\bar{G}$ over $\overline{\mathcal{K}}$, and $\pi$ is any subquotient of its $\bmod \ell$ reduction, then the supercuspidal support of $\pi$ is equal to the supercuspidal support of the $\bmod \ell$ reduction of the supercuspidal support of $\tilde{\pi}$.

Deligne-Lusztig theory provides a parameterization of the irreducible cuspidal representations of $\bar{G}$ over $\overline{\mathcal{K}}$ in terms of semisimple elements $s$ of $\bar{G}$ whose characteristic polynomials are irreducible, up to conjugacy. To a semisimple element $s$ (up to conjugacy), we associate a subset of the irreducible representations of $\bar{G}$ over $\overline{\mathcal{K}}$ as follows: let $\bar{M}_{s}$ be the minimal split Levi subgroup of $\bar{G}$ containing $s$. Then $\bar{M}_{s}$ is a product of general linear groups $\bar{G}_{n_{i}}$, 
and the factors $s_{i}$ of $s$ under this decomposition all have irreducible characteristic polynomials. We call the $s_{i}$ the 'irreducible factors' of $s$, and refer to an $s$ with only one irreducible factor as 'irreducible.' Thus each $s_{i}$ yields a cuspidal representation of $\bar{G}_{n_{i}}$, and hence $s$ yields a cuspidal representation $\pi^{\prime}$ of $\bar{M}_{s}$. Let $\mathcal{I}(s)$ be the set of irreducible representations of $\bar{G}$ over $\overline{\mathcal{K}}$ with cuspidal support $\left(\bar{M}_{s}, \pi^{\prime}\right)$. Note that if $s$ and $t$ are conjugate then the pair $\left(\bar{M}_{t}, \pi^{\prime \prime}\right)$ attached to $t$ is conjugate to the pair $\left(\bar{M}_{s}, \pi^{\prime}\right)$, so that $\mathcal{I}(s)=\mathcal{I}(t)$. More generally, if $\bar{M}$ is a Levi subgroup of $\bar{G}$, and $s$ is a semisimple conjugacy class in $\bar{M}$, we let $\mathcal{I}_{\bar{M}}(s)$ be the set of irreducible representations of $\bar{M}$ over $\overline{\mathcal{K}}$ whose cuspidal support is $\bar{M}$-conjugate to $\left(\bar{M}_{s}, \pi^{\prime}\right)$. We let $I_{s}$ denote the parabolic induction $i \frac{\bar{G}}{P} \pi^{\prime} ; I_{s}$ depends only on the conjugacy class of $s$, and the irreducible representations that appear with nonzero multiplicity in $I_{s}$ are precisely the elements of $\mathcal{I}(s)$.

We now recall the concept of a generic representation of $\bar{G}$. Let $\bar{U}$ be the unipotent radical of a Borel subgroup of $\bar{G}$, and let $\Psi: \bar{U} \rightarrow W(k)^{\times}$be a generic character. (For instance, if $U$ is the subgroup of upper triangular matrices with 1's on the diagonal, we can fix a nontrivial map $\psi$ of $\mathbb{F}_{q}^{+}$into $W(k)^{\times}$and set $\Psi(u)=\psi\left(u_{12}+\cdots+u_{n-1, n}\right)$.) We say an irreducible representation of $\bar{G}$ over $k$ (respectively $\overline{\mathcal{K}}$ ) is generic if its restriction to $\bar{U}$ contains a copy of $\Psi \otimes_{W(k)} k$ (respectively $\Psi \otimes_{W(k)} \overline{\mathcal{K}}$ ). By Frobenius reciprocity a representation is generic if and only if it admits a nontrivial map from c-Ind $\frac{\bar{G}}{U} \Psi$.

We summarize the relevant facts about generic representations that we will need below:

(1) If $\pi$ is an irreducible generic representation over $k$ (respectively $\overline{\mathcal{K}}$ ) then its restriction to $\bar{U}$ contains exactly one copy of $\Psi \otimes_{W(k)} k$ (respectively $\left.\Psi \otimes_{W(k)} \overline{\mathcal{K}}\right)$. (Uniqueness of Whittaker models.)

(2) Every cuspidal representation is generic.

(3) If $\bar{P}$ is a parabolic subgroup of $\bar{G}$, with Levi subgroup $\bar{M}$, and $\pi$ is an irreducible generic representation of $\bar{M}$, then $i \frac{\bar{G}}{P} \pi$ has a unique irreducible generic subquotient. (In particular, there is, up to isomorphism, a unique generic irreducible representation with given supercuspidal support.) On the other hand, if $\pi$ is irreducible but not generic, then $i \frac{\bar{G}}{P} \pi$ has no generic subquotient.

In light of these facts, for any semisimple element $s$ of $\bar{G}$, we let $\mathrm{St}_{s}$ denote the unique irreducible generic representation of $\bar{G}$ over $\overline{\mathcal{K}}$ that lies in $\mathcal{I}(s)$. (Of course, $\mathrm{St}_{s}$ only depends on $s$ up to conjugacy.) Note that $\mathrm{St}_{s}$ is a direct summand of $I_{s}$. It will be necessary to understand the behavior of $I_{s}$ and $\mathrm{St}_{s}$ under parabolic restriction. 
PROPOSITION 5.3. We have a decomposition:

$$
r_{\bar{G}}^{\bar{P}} \mathrm{St}_{s} \cong \bigoplus_{t} \mathrm{St}_{\bar{M}, t}
$$

where $t$ runs over a set of representatives for $\bar{M}$-conjugacy classes of semisimple elements of $\bar{M}$ that are $\bar{G}$-conjugate to $s$, and $\mathrm{St}_{\bar{M}, t}$ is the unique irreducible generic representation of $\bar{M}$ over $\overline{\mathcal{K}}$ that lies in $\mathcal{I}_{\bar{M}}(t)$.

Proof. By Frobenius reciprocity, for any irreducible representation $\sigma$ of $\bar{M}$, we have an isomorphism:

$$
\operatorname{Hom}_{\bar{M}}\left(\sigma, r_{\bar{G}}^{\bar{P}} \mathrm{St}_{s}\right)=\operatorname{Hom}_{\bar{G}}\left(i \frac{\bar{G}}{P} \sigma, \mathrm{St}_{s}\right) .
$$

As $\mathrm{St}_{s}$ is generic and irreducible, the right-hand side is zero unless $i \frac{\bar{G}}{P} \sigma$ has an irreducible generic summand; if this is the case then $\sigma$ is irreducible and generic, $i \frac{\bar{G}}{P} \sigma$ has a unique irreducible generic summand, so the right-hand side has dimension at most one. In particular, every irreducible summand of $r_{\bar{G}}^{\bar{P}} \mathrm{St}_{s}$ is generic and occurs with multiplicity one. Moreover, if the right-hand side is nonzero, then the cuspidal support of one (hence every) summand of $\sigma$ is given by $s$, so the $\bar{M}$-cuspidal support of $\sigma$ is given by a conjugacy class $t$ of $\bar{M}$ that is $\bar{G}$-conjugate to $s$.

We can rewrite this isomorphism as follows: Let $\bar{M}_{s}$ be the minimal split Levi of $\bar{G}$ containing $s$, so that $\mathrm{St}_{\bar{M}_{s}, s}$ is cuspidal. Fix a maximal torus of $\bar{M}$; then conjugating $s$ appropriately we may assume that it is also a maximal torus of $\bar{M}_{s}$. Consider the set $W\left(\bar{M}_{s}, \bar{M}\right)$ of elements $w$ of $W(\bar{G})$ such that $w \bar{M}_{s} w^{-1}$ lies in $\bar{M}$. Then $W\left(\bar{M}_{s}, \bar{M}\right)$ has a left action by $W(\bar{M})$ and a right action by the subgroup $W_{\bar{M}_{s}}(s)$ of $W(\bar{G})$ consisting of those $w$ in $W(\bar{G})$ such that $w \bar{M}_{s} w^{-1}=\bar{M}_{s}$ and $w s w^{-1}$ is $\bar{M}_{s}$-conjugate to $s$. Moreover, the map $s \mapsto w s w^{-1}$ then yields a bijection between $W(\bar{M}) \backslash W\left(\bar{M}_{s}, \bar{M}\right) / W_{\bar{M}}(s)$ and the set of $\bar{M}$-conjugacy classes $t$ of elements that are $\bar{G}$-conjugate to $s$. We thus obtain a decomposition:

PROPOSITION 5.4. We have a decomposition:

$$
r_{\bar{G}}^{\bar{P}} \mathrm{St}_{s} \cong \bigoplus_{w} \mathrm{St}_{\bar{M}, w s w^{-1}}
$$

where $w$ runs over a set of representatives for $W(M) \backslash W\left(\bar{M}_{s}, \bar{M}\right) / W_{\bar{M}}(s)$.

We will need to understand the compatibility of this decomposition with a decomposition of $r_{\bar{G}}^{\bar{P}} I_{s}$. We have: 
Proposition 5.5. There is a direct sum decomposition:

$$
r_{\bar{G}}^{\bar{P}} I_{s} \cong \bigoplus_{w} I_{\bar{M}, w s w^{-1}}
$$

where $w$ runs over a set of representatives for $W(M) \backslash W\left(\bar{M}_{s}, \bar{M}\right)$, and $I_{\bar{M}, w s w^{-1}}$ is the parabolic induction: $i_{w \bar{P}_{s} w^{-1} \cap \bar{M}} \mathrm{St}_{\bar{M}_{s}, s}^{w}$. Moreover, on a summand $\mathrm{St}_{\bar{M}, w^{\prime} s\left(w^{\prime}\right)^{-1}}$ of $r_{\bar{G}}^{\bar{P}} \mathrm{St}_{s}$, the map $r_{\bar{G}}^{\bar{P}} \mathrm{St}_{s} \rightarrow r_{\bar{G}}^{\bar{P}} I_{s}$ induces an injective map

$$
\mathrm{St}_{\bar{M}, w^{\prime} s\left(w^{\prime}\right)^{-1}} \rightarrow \bigoplus_{w} I_{\bar{M}, w s w^{-1}}
$$

Proof. This is a consequence of Mackey's induction-restriction formula, together with Proposition 5.3.

We now turn to considerations related to reduction modulo $\ell$. Given an irreducible cuspidal representation $\pi$ of $\bar{G}$ over $\overline{\mathcal{K}}$, its mod $\ell$ reduction is irreducible and cuspidal. Every cuspidal representation of $\bar{G}$ over $k$ arises by $\bmod$ $\ell$ reduction from some such $\pi$. If $\pi$ corresponds to a semisimple conjugacy class $s$, then the reduction $\bmod \ell$ of $\pi$ is supercuspidal if, and only if, the characteristic polynomial of the $\ell$-regular part $s^{\text {reg }}$ of $s$ is irreducible. Moreover, if $\pi$ and $\pi^{\prime}$ are irreducible cuspidal representations correspond to semisimple conjugacy classes $s$ and $s^{\prime}$, then the $\bmod \ell$ reductions of $\pi$ and $\pi^{\prime}$ coincide if, and only if, $s^{\text {reg }}=\left(s^{\prime}\right)^{\text {reg }}$. Thus the supercuspidal representations of $\bar{G}$ over $k$ are parameterized by $\ell$-regular semisimple conjugacy classes in $\bar{G}$ with irreducible characteristic polynomial, and the cuspidal representations of $\bar{G}$ over $k$ are parameterized by $\ell$-regular semisimple conjugacy classes $s^{\prime}$ such that there exists a semisimple conjugacy class $s$, with irreducible characteristic polynomial, such that $s^{\prime}=s^{\text {reg }}$.

Let $\pi$ be a cuspidal but not supercuspidal representation of $\bar{G}$ over $k$, and let $s^{\prime}$ be the corresponding semisimple element. Such an $s^{\prime}$, factors into $m$ identical irreducible factors $s_{0}^{\prime}$ for some $m$ dividing $n$. Moreover, the supercuspidal support of the $\pi$ that corresponds to $s^{\prime}$ is the tensor product of $m$ copies of the supercuspidal representation of $\bar{G}_{n / m}$ corresponding to $s_{0}^{\prime}$.

Fix an $\ell$-regular semisimple element $s^{\prime}$ of $\bar{G}$, and let $\mathcal{E}\left(s^{\prime}\right)$ be the union of the sets $\mathcal{I}(s)$ for those semisimple $s$ with $s^{\text {reg }}=s^{\prime}$. Let $e_{s^{\prime}}$ be the idempotent in $\overline{\mathcal{K}}[\bar{G}]$ that is the sum of the primitive idempotents $e_{\pi}$ for all $\pi$ in $\mathcal{E}\left(s^{\prime}\right)$. Then one has:

THEOREM 5.6. The element $e_{s^{\prime}}$ lies in $W(k)[\bar{G}]$.

Proof. This is an immediate consequence of [CE, Theorem 9.12]. 
Fix an irreducible cuspidal representation $\pi$ of $\bar{G}$ over $k$ that is not supercuspidal, corresponding to an $\ell$-regular semisimple element $s^{\prime}$ (up to conjugacy). The representation $\pi$ arises as the $\bmod \ell$ reduction of an irreducible cuspidal representation $\tilde{\pi}$ of $\pi$ over $\overline{\mathcal{K}}$; there is thus a semisimple element $s$ of $\bar{G}$, with irreducible characteristic polynomial, such that $s^{\prime}=s^{\mathrm{reg}}$. It is then easy to see that there exists an $m>1$ dividing $n$ such that, up to conjugacy, $s^{\prime}$ factors as a block matrix consisting of $m$ irreducible factors $s_{0}^{\prime}$. Moreover, $m$ lies in the set $\left\{1, e_{q}, \ell e_{q}, \ell^{2} e_{q}, \ldots\right\}$, where $e_{q}$ is the order of $q$ modulo $\ell$. The supercuspidal support of $\pi$ is the tensor product of $m$ copies of the supercuspidal representation $\pi_{0}$ corresponding to $s^{\prime}$.

PROPOSITION 5.7. Suppose we have an irreducible representation $\tilde{\pi}$ of $\bar{G}$ over $\mathcal{K}$ whose mod $\ell$ reduction contains $\pi$ as a subquotient. Let $s$ be the semisimple conjugacy class corresponding to the supercuspidal support of $\tilde{\pi}$. Then $s^{\mathrm{reg}}=s^{\prime}$.

Proof. The supercuspidal support of $\tilde{\pi}$ is given by the pair $\left(M_{s}, \tilde{\pi}_{M, s}\right)$, where $M_{s}$ is the minimal split Levi containing $s$ and $\tilde{\pi}_{M, s}$ is the corresponding supercuspidal representation of $M_{s}$ over $\overline{\mathcal{K}}$. The $\bmod \ell$ reduction $\tilde{\pi}$ is thus isomorphic to a subquotient of a parabolic induction $i \frac{\bar{G}}{P} \pi_{M, s}$, where $\pi_{M, s}$ is the $\bmod \ell$ reduction of $\tilde{\pi}_{M, s}$. By the above discussion, $\pi_{M, s}$ is the cuspidal representation of $M_{s}$ attached to $s^{\text {reg }}$, which has supercuspidal support $\left(M_{s^{\mathrm{reg}}}, \pi_{s^{\mathrm{reg}}}\right)$. Since $\pi$ is a subquotient of a parabolic induction of $\pi_{M, s}$, the supercuspidal support of $\pi$ is also $\left(M_{s^{\mathrm{reg}}}, \pi_{s^{\mathrm{reg}}}\right)$. In particular, $s^{\text {reg }}$ and $s^{\prime}$ represent the same conjugacy class.

Fix an irreducible cuspidal representation $\pi$ of $\bar{G}$ over $k$, and let $s^{\prime}$ be a representative of the corresponding $\ell$-regular semisimple conjugacy class. Let $\mathcal{P}_{\pi}$ be a projective envelope of $\pi$.

Proposition 5.8. The $W(k)[\bar{G}]$-module $e_{s^{\prime}} \operatorname{Ind}_{\bar{U}}^{\bar{G}} \Psi$ is a projective envelope of $\pi$, and hence is isomorphic to $\mathcal{P}_{\pi}$. In particular, $\mathcal{P}_{\pi} \otimes_{W(k)} \overline{\mathcal{K}}$ is isomorphic to the direct sum

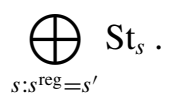

Moreover, the endomorphism ring $\operatorname{End}_{W(k)[\bar{G}]}\left(\mathcal{P}_{\pi}\right)$ is a reduced, commutative, free $W(k)$-module of finite rank.

Proof. The module $e_{s^{\prime}} \operatorname{Ind}_{\bar{U}}^{\bar{G}} \Psi$ is projective, as induction takes projectives to projectives. Suppose $\pi^{\prime}$ is an irreducible representation of $\bar{G}$ over $k$ that admits a nonzero map from $e_{s^{\prime}} \operatorname{Ind} \overline{\bar{G}} \Psi$. Then $\pi^{\prime}$ is generic and has supercuspidal support given by $s^{\prime}$, so $\pi^{\prime}=\pi$. Thus $e_{s^{\prime}} \operatorname{Ind} \frac{\bar{G}}{U} \Psi$ is a projective envelope of $\pi$. 
As $\mathcal{P}_{\pi}$ is free of finite rank over $W(k)$, so is $\operatorname{End}_{W(k)[\bar{G}]}\left(\mathcal{P}_{\pi}\right)$. Thus we have an embedding:

$$
\operatorname{End}_{W(k)[\bar{G}]}\left(\mathcal{P}_{\pi}\right) \rightarrow \operatorname{End}_{W(k)[\bar{G}]}\left(\mathcal{P}_{\pi}\right) \otimes_{W(k)} \overline{\mathcal{K}}
$$

The latter is the endomorphism ring of the module $e_{s^{\prime}} \operatorname{Ind}_{\bar{U}} \bar{G}\left(\Psi \otimes_{W(k)} \overline{\mathcal{K}}\right)$. This module is a direct sum of the generic representations $\tilde{\pi}$ of $\bar{G}$ over $\overline{\mathcal{K}}$ whose $\bmod \ell$ reduction contains $\pi$, each with multiplicity one. In particular, its endomorphism ring is reduced and commutative.

Let $\bar{P}$ be a parabolic subgroup of $\bar{G}$, let $\bar{U}$ be its unipotent radical, and let $\bar{M}$ be the corresponding Levi subgroup. We will need to understand the restriction $r_{\bar{G}} \bar{P} \mathcal{P}_{\pi}$. The following lemma is an immediate consequence of Proposition 5.3:

LEMMA 5.9. We have an isomorphism:

$$
r_{\bar{G}}^{\bar{P}} \mathcal{P}_{\pi} \otimes \overline{\mathcal{K}}=\bigoplus_{s: \mathrm{reg}^{\mathrm{res}}} \bigoplus_{t \sim s} \mathrm{St}_{\bar{M}, t}
$$

In particular, the endomorphism ring $\operatorname{End}_{W(k)[\bar{M}]}\left(r_{G}^{P} \mathcal{P}_{\pi}\right)$ is reduced and commutative.

Suppose that each block of $\bar{M}$ has size divisible by $n / m$. Then $s^{\prime}$ is conjugate to an element of $\bar{M}$, and this element is unique up to $\bar{M}$-conjugacy. Then $\mathrm{St}_{\bar{M}, s^{\prime}}$ is the unique generic representation of $\bar{M}$ determined by this conjugacy class in $\bar{M}$. Let $\mathcal{P}_{\bar{M}, s^{\prime}}$ be a projective envelope of $\mathrm{St}_{\bar{M}, s^{\prime}}$.

PROPOSITION 5.10. The restriction $r_{\bar{G}}^{\bar{P}} \mathcal{P}_{\pi}$ is zero unless each block of $\bar{M}$ has size divisible by $n / m$. When the latter occurs there is an isomorphism:

$$
r_{\bar{G}}^{\bar{P}} \mathcal{P}_{\pi} \cong \mathcal{P}_{\bar{M}, s^{\prime}}
$$

Proof. Every Jordan-Hölder constituent of $\mathcal{P}_{\pi}$ has supercuspidal support corresponding to a tensor product of $m$ copies of the representation with supercuspidal support $s_{0}^{\prime}$, and thus $r_{\bar{G}}^{\bar{P}} \mathcal{P}_{\pi}=0$ unless the condition on the block size of $M$ holds. When this condition does hold, $r_{\bar{G}}^{\bar{P}} \mathcal{P}_{\pi}$ is projective, and hence a direct sum of indecomposable projectives. Moreover, $i \frac{\bar{G}}{P} \mathrm{St}_{\bar{M}, s^{\prime}}$ contains a unique generic Jordan-Hölder constituent; this constituent is necessarily isomorphic to $\pi$, and thus yields a map $\mathcal{P}_{\pi} \rightarrow i \frac{\bar{G}}{P} \mathrm{St}_{\bar{M}, s^{\prime}}$. By Frobenius reciprocity we obtain a map:

$$
r_{\bar{G}}^{\bar{P}} \mathcal{P}_{\pi} \rightarrow \mathrm{St}_{\bar{M}, s^{\prime}}
$$


and therefore $r_{\bar{G}}^{\bar{P}} \mathcal{P}_{\pi}$ has a summand isomorphic to $\mathcal{P}_{\bar{M}, s^{\prime}}$. It is then easy to see that this is the only summand, by tensoring with $\overline{\mathcal{K}}$ and applying Lemma 5.9.

COROLlary 5.11. The map

$$
e_{s^{\prime}} Z(W(k)[\bar{G}]) \rightarrow \operatorname{End}_{W(k)[\bar{G}]}\left(\mathcal{P}_{\pi}\right)
$$

admits a section:

$$
\operatorname{End}_{W(k)[\bar{G}]}\left(\mathcal{P}_{\pi}\right) \rightarrow e_{s^{\prime}} Z(W(k)[\bar{G}])
$$

such that the composition:

$$
\operatorname{End}_{W(k)[\bar{G}]}\left(\mathcal{P}_{\pi}\right) \rightarrow e_{s^{\prime}} Z(W(k)[\bar{G}]) \rightarrow \operatorname{End}_{W(k)[\bar{G}]}\left(\mathcal{P}_{\pi}\right)
$$

is the identity.

Proof. The $W(k)[\bar{G}]$-module $e_{s^{\prime}} W(k)[\bar{G}]$ is a projective module, and is therefore a direct sum (with multiplicities) of projective envelopes of modules in the block containing $\pi$. Any such module $\pi^{\prime}$ has supercuspidal support corresponding to $\left(s_{0}^{\prime}\right)^{m}$, and hence cuspidal support of the form $\left(\bar{M}, \pi_{\bar{M}, s^{\prime}}\right)$ for some Levi $\bar{M}$ of $G$. It is easy to see that $i \frac{\bar{G}}{P} \mathcal{P}_{\bar{M}, s^{\prime}}$ is then a projective envelope of $\pi^{\prime}$. Thus $e_{s^{\prime}} W(k)[\bar{G}]$ is isomorphic to a direct sum of modules of the form $i \frac{\bar{G}}{P} \mathcal{P}_{\bar{M}, s^{\prime}}$ for various $\bar{M}$.

As $\mathcal{P}_{\bar{M}, s^{\prime}}$ is isomorphic to a parabolic restriction of $\mathcal{P}_{\pi}$, the endomorphisms of $\mathcal{P}_{\pi}$ act naturally on $\mathcal{P}_{\bar{M}, s^{\prime}}$, and hence on $i \frac{\bar{G}}{\bar{P}} \mathcal{P}_{\bar{M}, s^{\prime}}$. This gives an action of $\operatorname{End}_{W(k)[\bar{G}]}\left(\mathcal{P}_{\pi}\right)$ on $e_{s^{\prime}} W(k)[\bar{G}]$, via elements of the center of $e_{s^{\prime}} W(k)[\bar{G}]$. The resulting map

$$
\operatorname{End}_{W(k)[\bar{G}]}\left(\mathcal{P}_{\pi}\right) \rightarrow e_{s^{\prime}} Z(W(k)[\bar{G}])
$$

is the desired section.

We now obtain more precise results about $\mathcal{P}_{\pi}$ under the additional hypothesis that $\ell>n$.

Let $\Phi_{e}(x)$ be the eth cyclotomic polynomial.

LEMMA 5.12. Let $r$ be the order of $q \bmod \ell$, and suppose there exists an $e$ not equal to $r$ such that $\ell$ divides $\Phi_{e}(q)$. Then $\ell$ divides $e$.

Proof. As $q$ has exact order $r \bmod \ell$, it is clear that $\ell$ divides $\Phi_{r}(q)$, and also that $r$ divides $e$. The polynomial $x^{e}-1$ is divisible by $\Phi_{r}(x) \Phi_{e}(x)$; as $q$ is a root of both of these polynomials $\bmod \ell$ we see that $x^{e}-1$ has a double root $\bmod \ell$, and so $\ell$ divides $e$ as required.

It follows that for $\ell>n$, there exists at most one $e$ dividing $n$ with $\Phi_{e}(q)$ divisible by $\ell$. 
As a result, one has:

LEMMA 5.13. Let $s^{\prime}$ be an $\ell$-regular semisimple element of $\mathrm{GL}_{n}\left(\mathbb{F}_{q}\right)$, whose characteristic polynomial is reducible, and suppose that there exists a semisimple element $s$ of $\mathrm{GL}_{n}\left(\mathbb{F}_{q}\right)$ with irreducible characteristic polynomial such that $s^{\prime}=s^{\mathrm{reg}}$. Suppose also that $\ell>n$. Then for any $s$ such that $s^{\prime}=s^{\mathrm{reg}}$, either $s=s^{\prime}$ or the characteristic polynomial of $s$ is irreducible.

Proof. Fix a semisimple element $s$ with irreducible characteristic polynomial such that $s^{\prime}=s^{\text {reg }}$. Then $s$ is contained in a subgroup of $G_{n}$ isomorphic to $\mathbb{F}_{q^{n}}^{\times}$, and (when considered as an element of $\mathbb{F}_{q^{n}}^{\times}$), $s$ generates $\mathbb{F}_{q^{n}}$ over $\mathbb{F}_{q}$. We regard $s$ and $s^{\prime}$ as elements of $\mathbb{F}_{q^{n}}$. Write $s=s^{\prime} s_{\ell}$, where $s_{\ell}$ is $\ell$-power torsion. Then there are integers $e_{\ell}$ and $e^{\prime}$ dividing $n$ such that $s^{\prime}$ generates $\mathbb{F}_{q^{e^{\prime}}}$ over $\mathbb{F}_{q}$ and $s_{\ell}$ generates $\mathbb{F}_{q^{e_{\ell}}}$ over $\mathbb{F}_{q}$. The least common multiple of $e^{\prime}$ and $e_{\ell}$ is equal to $n$. Note that $e^{\prime}$ is not equal to $n$, as this would imply that the characteristic polynomial of $s^{\prime}$ was irreducible.

In particular, $e_{\ell}$ cannot be equal to 1 , so $e_{\ell}$ is a nontrivial root of unity. Thus $\ell$ divides $\Phi_{e_{\ell}}(q)$, and hence $e_{\ell}$ is the unique $m<n$ such that $\ell$ divides $\Phi_{m}(q)$.

Now if $s$ is an arbitrary element with $s^{\text {reg }}=s$, we can write $s$ as $s^{\prime} s_{\ell}$ for some $\ell$-power root of unity $s_{\ell}$. Either $s_{\ell}$ is trivial or $s_{\ell}$ lies in $\mathbb{F}_{q^{e_{\ell}}}$; as we know that the least common multiple of $e^{\prime}$ and $e_{\ell}$ is $n$ the latter case implies that $s$ generates $\mathbb{F}_{q^{n}}$ over $\mathbb{F}_{q}$, as required.

In particular, if $\ell>n$, and $\sigma$ is a representation that is cuspidal but not supercuspidal, corresponding to a semisimple element $s^{\prime}$, then every irreducible representation $\tilde{\sigma}$ of $G_{n}$ over $\overline{\mathcal{K}}$ whose mod $\ell$ reduction contains $\sigma$ is either a supercuspidal lift of $\sigma$, or has supercuspidal support given by $s^{\prime}$. We thus have:

COROLlary 5.14. For $\ell>n$, and $\pi$ cuspidal but not supercuspidal, we have:

$$
\mathcal{P}_{\sigma} \otimes \overline{\mathcal{K}} \cong \mathrm{St}_{s^{\prime}} \oplus \bigoplus_{\tilde{\sigma}} \tilde{\sigma}
$$

where $\tilde{\sigma}$ runs over the supercuspidal representations lifting $\sigma$.

\section{Structure of $\mathcal{P}_{K, \tau}$ in depth zero}

The goal of the next four sections will be to apply the finite group theory of Section 5 to understand the structure of $\mathcal{P}_{K, \tau}$. Recall that, by definition, we have

$$
\mathcal{P}_{K, \tau}=\mathrm{c}-\operatorname{Ind}_{K}^{G} \tilde{\kappa} \otimes \mathcal{P}_{\sigma} .
$$


The representation $\sigma$ is inflated from a cuspidal representation of $\mathrm{GL}_{n / e f}\left(\mathbb{F}_{q^{f}}\right)$; such a representation is of the form $\mathrm{St}_{s^{\prime}}$ for some semisimple $\ell$-regular element $s^{\prime}$ of $\mathrm{GL}_{n / e f}\left(\mathbb{F}_{q^{f}}\right)$. For conciseness we abbreviate $\mathrm{GL}_{n / e f}\left(\mathbb{F}_{q^{f}}\right)$ by $\bar{G}$ for this section.

The decomposition

$$
\mathcal{P}_{\sigma} \otimes \overline{\mathcal{K}} \cong \bigoplus_{s} \mathrm{St}_{s}
$$

of Proposition 5.8 gives rise to a decomposition:

$$
\mathcal{P}_{K, \tau} \otimes \overline{\mathcal{K}} \cong \bigoplus_{s} \mathrm{c}-\operatorname{Ind}_{K}^{G} \tilde{\kappa} \otimes \mathrm{St}_{s},
$$

where $s$ runs over a set of representatives for the $\bar{G}$-conjugacy classes of semisimple elements $s$ whose $\ell$-regular part is conjugate to $s^{\prime}$.

DEFINITION 6.1. Let $(K, \kappa \otimes \sigma)$ be a maximal distinguished cuspidal $k$-type. A generic pseudotype attached to $(K, \kappa \otimes \sigma)$ is a $\overline{\mathcal{K}}$-type of the form $\left(K, \tilde{\kappa} \otimes \mathrm{St}_{s}\right)$, where $s$ is a semisimple element of $\bar{G}$ whose $\ell$-regular part is conjugate to $s^{\prime}$.

If $\mathrm{St}_{s}$ is cuspidal, then the generic pseudotype $\left(K, \tilde{\kappa} \otimes \mathrm{St}_{s}\right)$ is a maximal distinguished $\overline{\mathcal{K}}$-type, whose $\bmod \ell$ reduction is the type $(K, \kappa \otimes \sigma)$. In general a generic pseudotype is not a type in the usual sense of the word; we will see that in some sense the Hecke algebras attached to generic pseudotypes are analogues of spherical Hecke algebras. (In particular, they can be identified with centers of Hecke algebras attached to types.)

In this section and Section 8 we will prove several basic results about the structure of representations induced from generic pseudotypes, as well as certain natural $W(k)[G]$-submodules of these representations.

Our approach makes use of the theory of $G$-covers, which can be found in [BK1] for a field of characteristic zero, and [V2] over a general base ring. We largely follow the presentation of [V2, Section II]. Let $P=M U$ be a parabolic subgroup of $G$, with Levi subgroup $M$, unipotent radical $U$, and opposite parabolic subgroup $P^{\circ}=M U^{\circ}$. Let $(K, \tau)$ be an $R$-type of $G$, and let $K_{M}, K^{+}, K^{-}$denote the intersections $K \cap M, K \cap U ; K \cap U^{\circ}$, respectively. Let $\tau_{M}$ be the restriction of $\tau$ to $K_{M}$.

DEFINITION 6.2. The pair $(K, \tau)$ is decomposed with respect to $P$ if $K=K^{-} K_{M} K^{+}$, and $\tau$ is trivial on $K^{+}$and $K^{-}$. If $\tau$ is the trivial representation we will sometimes say that $K$ is decomposed with respect to $P$.

The key example that motivates this definition is as follows: Let $P$ be a standard parabolic subgroup of $G$, with Levi subgroup $M$, and let $\bar{P}$ and $\bar{M}$ be the images 
of $P \cap \mathrm{GL}_{n}\left(\mathcal{O}_{F}\right)$ and $M \cap \mathrm{GL}_{n}\left(\mathcal{O}_{F}\right)$ under the reduction map $\mathrm{GL}_{n}\left(\mathcal{O}_{F}\right) \rightarrow \bar{G}=$ $\mathrm{GL}_{n}\left(\mathbb{F}_{q}\right)$. If we then take $K_{P}$ to be the preimage of $\bar{P}$ in $\mathrm{GL}_{n}\left(\mathcal{O}_{F}\right)$, and let $\tau$ be any representation of $K_{P}$ inflated from $\bar{M}$ via the maps:

$$
K_{P} \rightarrow \bar{P} \rightarrow \bar{M}
$$

then one checks easily that the pair $\left(K_{P}, \tau\right)$ is decomposed with respect to $P$. We will be concerned primarily with this example for the majority of this section and the next.

Returning for a moment to the general situation, suppose that $(K, \tau)$ is decomposed with respect to $P$, and let $\lambda$ be an element of $M$. Following [V2], II.4, we say that $\lambda$ is positive if $\lambda K^{+} \lambda^{-1}$ is contained in $K^{+}$, and $\lambda^{-1} K^{-} \lambda$ is contained in $\lambda$. We say $\lambda$ is negative if $\lambda^{-1}$ is positive.

We say that $\lambda$ is strictly positive if the following two conditions hold:

- For any pair of open compact subgroups $U_{1}, U_{2}$ of $U$, there exists a positive $m$ such that $\lambda^{m} U_{1} \lambda^{-m}$ is contained in $U_{2}$.

- For any pair of open compact subgroups $U_{1}^{\circ}, U_{2}^{\circ}$ of $U^{\circ}$, there exists a positive $m$ such that $\lambda^{-m} U_{1}^{\circ} \lambda^{m}$ is contained in $U_{2}^{\circ}$.

The discussion of [ $\mathbf{V} 2$, II.3] shows that when $(K, \tau)$ is decomposed with respect to $P$, there is a natural $R$-linear injection:

$$
T^{\prime}: H\left(M, K_{M}, \tau_{M}\right) \rightarrow H(G, K, \tau),
$$

that takes every element of $H\left(M, K_{M}, \tau_{M}\right)$ supported on $K_{M} \lambda K_{M}$ to an element supported on $K \lambda K$. Moreover, let $H\left(M, K_{M}, \tau_{M}\right)^{+}$be the subalgebra of $H\left(M, K_{M}, \tau_{M}\right)$ consisting of elements supported on double cosets $K_{M} \lambda K_{M}$ with $\lambda$ positive. Then the restriction $T^{+}$of $T^{\prime}$ to $H\left(M, K_{M}, \tau_{M}\right)^{+}$is an algebra homomorphism.

We now have the following result:

Proposition 6.3 [V2, II.6]. Suppose there exists a central, strictly positive element $\lambda$ of $M$ such that $T^{+}\left(1_{K_{M} \lambda K_{M}}\right)$ is an invertible element of $H(G, K, \tau)$, where $1_{K_{M} \lambda K_{M}}$ is the unique element of $H\left(M, K_{M}, \tau_{M}\right)$ that is supported on $K_{M} \lambda K_{M}$, and whose values at $\lambda$ is the identity endomorphism of $\tau_{M}$. Then $T^{+}$ extends uniquely to an algebra map:

$$
T: H\left(M, K_{M}, \tau_{M}\right) \rightarrow H(G, K, \tau) .
$$

If $(K, \tau)$ is decomposed with respect to $P$, and the hypothesis of Proposition 6.3 is satisfied, we say that $(K, \tau)$ is a $G$-cover of $\left(K_{M}, \tau_{M}\right)$. We then have: 
THEOREM 6.4 [BK3, page 55]. Suppose that $(K, \tau)$ is a $G$-cover of $\left(K_{M}, \tau_{M}\right)$, with $\tau$ and $\tau_{M}$ defined over $\overline{\mathcal{K}}$, and that the functor $\operatorname{Hom}_{K}(\tau,-)$ (respectively $\left.\operatorname{Hom}_{K_{M}}\left(\tau_{M},-\right)\right)$ is an equivalence of categories between a block of $\operatorname{Rep}_{\overline{\mathcal{K}}}(G)$ (respectively $\left.\operatorname{Rep}_{\overline{\mathcal{K}}}(M)\right)$ and the category of right $H(G, K, \tau)$-modules (respectively $H\left(M, K_{M}, \tau_{M}\right)$-modules).

Then for any $\overline{\mathcal{K}}[M]$-module $\Pi$, we have an isomorphism of $H(G, K, \tau)$ modules:

$$
\operatorname{Hom}_{K}\left(\tau, i_{P}^{G} \Pi\right) \cong \operatorname{Hom}_{H\left(M, K_{M}, \tau_{m}\right)}\left(H(G, K, \tau), \operatorname{Hom}_{K_{M}}\left(\tau_{M}, \Pi\right)\right)
$$

The first goal of this section is to understand the $\overline{\mathcal{K}}[G]$-module c-Ind ${ }_{K}^{G} \tilde{\kappa} \otimes \mathrm{St}_{s}$, in the so-called depth zero case where $K$ is the subgroup $\mathrm{GL}_{n}\left(\mathcal{O}_{F}\right)$ of $G$ and $\tilde{\kappa}$ is trivial. Let $\bar{M}$ be the minimal split Levi of $\bar{G}$ containing $s$; conjugating $s$ if necessary we assume that $\bar{M}$ is standard. Then there is a unique standard Levi subgroup $M$ of $G$ such that the image of $M \cap \mathrm{GL}_{n}\left(\mathcal{O}_{F}\right)$ in $\bar{G}$ is $\bar{M}$. We also fix a standard parabolic $P$ with Levi subgroup $M$, and let $\bar{P}$ be the image of $P \cap \mathrm{GL}_{n}\left(\mathcal{O}_{F}\right)$ in $\bar{G}$. We then set $K_{M}=K \cap M$ and let $K_{P}$ be the preimage of $\bar{P}$ in $K$. We regard $\mathrm{St}_{s}$ as a representation of $K$ via inflation, and $\mathrm{St}_{\bar{M}, s}$ as a representation of $K_{M}$ or $K_{P}$ (depending on context) via inflation from $\bar{M}$.

There is then a close relationship between the pair $\left(K_{P}, \mathrm{St}_{\bar{M}, s}\right)$ and the pair $\left(K, \mathrm{St}_{s}\right)$. Indeed, we have an isomorphism:

$$
\mathrm{c}-\operatorname{Ind}_{K_{P}}^{G} \mathrm{St}_{\bar{M}, s} \cong \mathrm{c}-\operatorname{Ind}_{K}^{G} i \frac{\bar{G}}{P} \mathrm{St}_{\bar{M}, s}
$$

and (since $\mathrm{St}_{s}$ is a direct summand of $i \frac{\bar{G}}{P} \mathrm{St}_{\bar{M}, s}$ ) it follows that c-Ind ${ }_{K}^{G} \mathrm{St}_{s}$ is a direct summand of c-Ind $K_{P}^{G} \mathrm{St}_{\bar{M}, s}$.

On the other hand, the pair $\left(K_{P}, \mathrm{St}_{\bar{M}, s}\right)$ is decomposed with respect to $P$, and indeed, one has:

THEOREM 6.5. The pair $\left(K_{P}, \mathrm{St}_{\bar{M}, s}\right)$ is a G-cover of the pair $\left(K_{M}, \mathrm{St}_{\bar{M}, s}\right)$. Moreover, these pairs satisfy the hypotheses of Theorem 6.4.

Proof. That the pair in question is a $G$-over follows from [BK3, Theorem 7.2], or, alternatively, from our description of the Hecke algebra $H\left(G, K_{P}, \mathrm{St}_{\bar{M}, s}\right)$ below.

By construction, $\left(K_{M}, \mathrm{St}_{\bar{M}, s}\right)$ is a maximal distinguished cuspidal $M$-type, and hence $\operatorname{Hom}_{K_{M}}\left(\mathrm{St}_{\bar{M}, s},-\right)$ is an equivalence of categories from the block of $\operatorname{Rep}_{\overline{\mathcal{K}}}(M)$ corresponding to the cuspidal type $\left(K_{M}, \mathrm{St}_{\bar{M}, s}\right)$ to the category of right $H\left(M, K_{M}, \mathrm{St}_{\bar{M}, s}\right)$-modules. It then follows from [BK2, Theorem 8.3] that $\operatorname{Hom}_{K_{P}}\left(\operatorname{St}_{\bar{M}, s},-\right)$ is an equivalence on a block of $\operatorname{Rep}_{\overline{\mathcal{K}}}(G)$. 
Let $\pi$ be an irreducible cuspidal $\overline{\mathcal{K}}$-representation of $M$ containing the type $\left(K_{M}, \mathrm{St}_{\bar{M}, s}\right)$. As an immediate consequence of the above theorem and Theorem 6.4 we deduce:

COROLLARY 6.6. The representations $\mathrm{c}-\mathrm{Ind}_{K_{P}}^{G} \mathrm{St}_{\bar{M}, s}$ and $\mathrm{c}-\mathrm{Ind}_{K}^{G} \mathrm{St}_{s}$ are objects of the block $\operatorname{Rep}_{\overline{\mathcal{K}}}(G)_{(M, \pi)}$.

We now turn to the question of computing the endomorphism ring of the induction $\mathrm{c}-\mathrm{Ind}_{K_{P}}^{G} \mathrm{St}_{\bar{M}, s}$. This ring is the Hecke algebra $H\left(G, K_{P}, \mathrm{St}_{\bar{M}, s}\right)$. The Mackey formula gives an isomorphism:

$$
H\left(G, K_{P}, \mathrm{St}_{\bar{M}, s}\right) \cong \bigoplus_{g} I_{g}\left(\mathrm{St}_{\bar{M}, s}\right)
$$

where $g$ runs over a set of representatives for the double cosets $K_{P} g K_{P}$. Without loss of generality we may restrict our attention to $g$ of the form $w t$, where $w$ is a permutation matrix and $t$ lies in the standard maximal torus $T$ of $G$. We may further assume that every entry of $t$ is a power of a fixed uniformizer $\varpi$ of $F$. Let $Z$ be the subgroup of $T$ consisting of elements $t$ such that every entry of $t$ is a power of $\varpi$.

We then have:

PROPOSITION 6.7. Let $W_{\bar{M}}(s)$ be the group of permutation matrices $w$ that normalize $\bar{M}$, and such that $w s w^{-1}$ is $\bar{M}$-conjugate to $s$. Let $g$ have the form $w t$, with $w$ a permutation matrix and $t \in Z$. Then $I_{g}\left(\mathrm{St}_{\bar{M}, s}\right)$ is one-dimensional if $w$ is in $W_{\bar{M}}(s)$ and $t$ is in the center $Z_{M}$ of $M$, and zero-dimensional otherwise.

Proof. Suppose first that $t$ does not lies in $Z_{M}$, or that $w$ does not normalize $M$. Then there is a unipotent subgroup $\bar{U}$ of $\bar{M}$, contained in the image of the composition:

$$
K_{P} \cap g K_{P} g^{-1} \rightarrow K \rightarrow \bar{G}
$$

that acts trivially on $\mathrm{St}_{\bar{M}, s}^{g}$. An element of $I_{g}\left(\mathrm{St}_{\bar{M}, s}\right)$ is, by definition, a $K_{P} \cap$ $g K_{P} g^{-1}$-equivariant map from $\mathrm{St}_{\bar{M}, s}$ to $\mathrm{St}_{\bar{M}, s}^{g}$; such a map must factor through the $\bar{U}$-coinvariants of $\mathrm{St}_{\bar{M}, s}$. $\mathrm{As}_{\bar{M}, s}$ is a cuspidal representation of $\bar{M}$, this space of coinvariants vanishes.

Now suppose that $t$ lies in $Z_{M}$ and $w$ normalizes $M$. Then the image of $K_{P} \cap g K_{P} g^{-1}$ under reduction from $\mathrm{GL}_{n}\left(\mathcal{O}_{F}\right)$ to $\bar{G}$ contains $\bar{M}$, and so we have an isomorphism:

$$
\operatorname{Hom}_{K_{P} \cap g K_{P} g^{-1}}\left(\mathrm{St}_{\bar{M}, s}, \mathrm{St}_{\bar{M}, s}^{g}\right) \cong \operatorname{Hom}_{\bar{M}}\left(\mathrm{St}_{\bar{M}, s}, \mathrm{St}_{\bar{M}, w s w^{-1}}\right) .
$$

The latter is one-dimensional if, and only if, $w$ lies in $W_{\bar{M}}(s)$ and zero otherwise. 
The characteristic polynomial of $s$ factors as a product of irreducible polynomials $f_{1}^{m_{1}} \ldots f_{r}^{m_{r}}$; this induces a factorization of $W_{\bar{M}}(s) / W(M)$ as a product of symmetric groups $S_{m_{i}}$, and a parallel factorization of $Z$ as a product of (free abelian) groups $Z_{i}$, such that the action of $W_{\bar{M}}(s) / W(M)$ on $Z_{i}$ factors through $S_{m_{i}}$ for all $i$. We may regard elements of $Z_{i}$ in two ways: first, they are elements of $Z$, and can thus be considered as $n$ by $n$ diagonal matrices whose elements are powers of $\varpi$. On the other hand, if we fix an identification of the quotient $S_{m_{i}}$ of $W_{\bar{M}}(s)$ with the Weyl group of $\mathrm{GL}_{m_{i}}\left(F_{i}\right)$, where $F_{i}$ is the unramified extension of $F$ of degree $d_{i}$, and let $Z_{i}^{\prime}$ denote the group of diagonal matrices in $\mathrm{GL}_{m_{i}}\left(F_{i}\right)$ whose entries are powers of $\varpi$, then there is a unique identification of $Z_{i}$ with $Z_{i}^{\prime}$ that is compatible with the actions of $S_{m_{i}}$ on $Z_{i}$ and the Weyl group of $\mathrm{GL}_{m_{i}}\left(F_{i}\right)$ on $Z_{i}^{\prime}$.

If we make these identifications, then the subspace $H_{i}$ of $H\left(G, K_{P}, \mathrm{St}_{\bar{M}, s}\right)$ supported on double cosets of the form $K_{P} w z K_{P}$, with $z \in Z_{i}$ and $w \in S_{m_{i}}$ is a subalgebra, and in fact a familiar one. Indeed, the construction of [BK1, 5.6], gives an isomorphism from the affine Hecke algebra $H\left(q, m_{i}\right)$ (regarded as the Hecke algebra $H\left(\mathrm{GL}_{m_{i}}\left(F_{i}\right), I\right)$, where $I$ is the Iwahori subgroup of $\mathrm{GL}_{m_{i}}\left(F_{i}\right)$ ) to $H_{i}$. This isomorphism is support-preserving, in the sense that it identifies the one-dimensional subspace of $H\left(\mathrm{GL}_{m_{i}}\left(F_{i}\right), I\right)$ supported on $I w z I$, for $z \in Z_{i}$ and $w$ in the Weyl group of $\mathrm{GL}_{m_{i}}\left(F_{i}\right)$ with the subspace of $H_{i}$ supported on $K_{P} w z K_{P}$. (This isomorphism depends on certain choices, but these will be irrelevant for our purposes.)

In this way we find that $H\left(G, K_{P}, \mathrm{St}_{\bar{M}, s}\right)$ is a tensor product of Iwahori Hecke algebras $H_{i}$. We will now relate this to $H\left(G, K, \mathrm{St}_{s}\right)$. We have seen that c-Ind ${ }_{K}^{G} \mathrm{St}_{s}$ is a summand of c-Ind $K_{P}^{G} \mathrm{St}_{\bar{M}, s}$; in particular any central endomorphism of the latter commutes with projection onto c-Ind ${ }_{K}^{G} \mathrm{St}_{s}$, and hence induces an endomorphism of c-Ind ${ }_{K}^{G} \mathrm{St}_{s}$. We thus have a natural map:

$$
Z\left(H\left(G, K_{P}, \mathrm{St}_{\bar{M}, s}\right)\right) \rightarrow H\left(G, K, \mathrm{St}_{s}\right) .
$$

Moreover, since we can identify $H\left(G, K_{P}, \mathrm{St}_{\bar{M}, s}\right)$ with $H\left(G, K, i \frac{\bar{G}}{P} \mathrm{St}_{\bar{M}, s}\right)$, this natural map is support-preserving, in the sense that an element of the domain supported on a union of double cosets $K_{P} g_{i} K_{P}$ maps to an element of $H\left(G, K, \mathrm{St}_{s}\right)$ supported on the union of the double cosets $K g_{i} K$.

Moreover, we have:

Proposition 6.8. The map $Z\left(H\left(G, K_{P}, \mathrm{St}_{\bar{M}, s}\right)\right) \rightarrow H\left(G, K, \mathrm{St}_{s}\right)$ is injective.

Proof. As c- $\operatorname{Ind}_{K}^{G} \mathrm{St}_{s}$ is a direct summand of c-Ind ${ }_{K}^{G} i \frac{\bar{G}}{P} \mathrm{St}_{\bar{M}, s}$, any endomorphism of the former extends by zero to an endomorphism of the latter. This allows us to view $H\left(G, K, \mathrm{St}_{s}\right)$ as a $Z\left(H\left(G, K, i \frac{\bar{G}}{P} \mathrm{St}_{\bar{M}, s}\right)\right.$-submodule of $H\left(G, K, i \frac{\bar{G}}{P} \mathrm{St}_{\bar{M}, s}\right)$; 
from this point of view the claim is that this submodule is not annihilated by any nonzero element of $Z\left(H\left(G, K, i \frac{\bar{G}}{P} \mathrm{St}_{\bar{M}, s}\right)\right)$. But $H\left(G, K, \tilde{\kappa} \otimes i \frac{\bar{G}}{P} \mathrm{St}_{\bar{M}, s}\right)$ is a tensor product of affine Hecke algebras; in particular (for instance, by Bernstein's presentation of $H(q, n)[\mathrm{Lu}])$, it is free over its center and its center is a domain. Thus no element of $H\left(G, K, i \frac{\bar{G}}{P} \mathrm{St}_{\bar{M}, s}\right)$ is annihilated by any element of the center of $H\left(G, K, i \frac{\bar{G}}{P} \mathrm{St}_{\bar{M}, s}\right)$.

In fact, we will show that this map is an isomorphism. In light of the injectivity shown above, and the fact that this map is support-preserving, this amounts to a dimension count.

Let $z$ be an element of $Z$, and let $\tilde{P}_{z}$ be the intersection: $\operatorname{GL}_{n}\left(\mathcal{O}_{F}\right) \cap$ $z \mathrm{GL}_{n}\left(\mathcal{O}_{F}\right) z^{-1}$. Then the image of $\tilde{P}_{z}$ under the projection $\mathrm{GL}_{n}\left(\mathcal{O}_{F}\right) \rightarrow \bar{G}$ is a parabolic subgroup $\bar{P}_{z}$ of $\bar{G}$; this parabolic subgroup contains a standard Levi $\bar{M}_{z}$.

Since $G$ is the union of the double cosets $K z K$, for $z \in Z$, it suffices to compute $H\left(G, K, \mathrm{St}_{s}\right)_{K z K} \cong I_{z}\left(\mathrm{St}_{s}\right)$ for all such $z$. By definition, we have

$$
I_{z}\left(\mathrm{St}_{s}\right)=\operatorname{Hom}_{\tilde{P}_{z}}\left(\mathrm{St}_{s}, \mathrm{St}_{s}^{z}\right)=\operatorname{Hom}_{\bar{P}_{z}}\left(\mathrm{St}_{s}, \mathrm{St}_{s}^{z}\right),
$$

where the last equality follows by noting that the kernel of the map $\tilde{P}_{z} \rightarrow \bar{P}_{z}$ acts trivially on both $\mathrm{St}_{s}$ and $\mathrm{St}_{s}^{z}$. Let $\bar{U}_{z}$ be the unipotent radical of $\bar{P}_{z}$. Then for any $u \in \tilde{P}_{z}$ that maps to $\bar{U}_{z}$, the element $z^{-1} u z$ acts trivially on $\mathrm{St}_{s}$, and so $u$ acts trivially on $\mathrm{St}_{s}^{z}$. In particular, any $\bar{P}_{z}$-equivariant map from $\mathrm{St}_{s}$ to $\mathrm{St}_{s}^{z}$ factors through the $\bar{U}_{z}$-coinvariants of $\mathrm{St}_{s}$; this space of coinvariants is precisely $r_{\bar{G}}^{\bar{P}_{z}} \mathrm{St}_{s}$. A parallel argument shows that the image of this map lies in a submodule of $\mathrm{St}_{s}^{z}$ that is isomorphic to $r_{\bar{G}}^{\bar{P}_{z}} \mathrm{St}_{s}$. In this way an element of $I_{z}\left(\mathrm{St}_{s}\right)$ gives rise to an element of $\operatorname{End}_{\bar{M}_{z}}\left(r_{\bar{G}}^{\bar{P}_{z}} \mathrm{St}_{s}\right)$, and the resulting map

$$
I_{z}\left(\mathrm{St}_{s}\right) \rightarrow \operatorname{End}_{\bar{M}_{z}}\left(r_{\bar{G}}^{\bar{P}_{z}} \mathrm{St}_{s}\right)
$$

is an isomorphism.

By Proposition 5.3 we have a direct sum decomposition:

$$
r_{\bar{G}}^{\bar{P}_{z}}\left(\mathrm{St}_{s}\right) \cong \bigoplus_{w} \mathrm{St}_{\bar{M}_{z}, w s w^{-1}}
$$

where $w$ runs over a set of representatives for $W\left(\bar{M}_{z}\right) \backslash W\left(\bar{M}, \bar{M}_{z}\right) / W_{\bar{M}}(s)$, and $\mathrm{St}_{\bar{M}_{z}, w s w^{-1}}$ is isomorphic to $\mathrm{St}_{\bar{M}_{z}, w^{\prime} s\left(w^{\prime}\right)^{-1}}$ if, and only if $w$ and $w^{\prime}$ lie in the same double coset. We thus find:

Proposition 6.9. For $z \in Z$, the dimension of $H\left(G, K, \mathrm{St}_{s}\right)_{K z K}$ is equal to the cardinality of the quotient $W\left(\bar{M}_{z}\right) \backslash W\left(\bar{M}, \bar{M}_{z}\right) / W_{\bar{M}}(s)$. 
It remains to compute the dimension of $Z\left(H\left(G, K_{P}, \mathrm{St}_{\bar{M}, s}\right)\right)$ supported on $K z K$. As $H\left(G, K_{P}, \mathrm{St}_{\bar{M}, s}\right)$ is a tensor produce of Iwahori Hecke algebras, it is first useful to observe:

LEMMA 6.10. For any element $z$ of $Z_{i}^{\prime}$, the subspace elements of $H\left(\mathrm{GL}_{m_{i}}\left(F_{i}\right), I\right)$ that are central and supported on the union of the double cosets I wzw'I, for $w$, $w^{\prime}$ in the Weyl group of $\mathrm{GL}_{m_{i}}\left(F_{i}\right)$, is one-dimensional. Moreover, the sum of these spaces as $z$ varies is the entire center of this Hecke algebra.

Proof. We are grateful to an anonymous referee for pointing out that this is essentially the content of [D1, Corollary 3.1]. We include an alternative argument for completeness.

Let $J$ be the subgroup $\mathrm{GL}_{m_{i}}\left(\mathcal{O}_{F_{i}}\right)$ of $\mathrm{GL}_{m_{i}}\left(F_{i}\right)$. Then the induction c-Ind ${ }_{I}^{J} 1$ contains the trivial character of $J$, and so c- $\operatorname{Ind}_{J}^{\mathrm{GL}_{m_{i}}\left(F_{i}\right)} 1$ is a direct summand of c-Ind $\mathrm{GL}_{I}^{\mathrm{GL}_{i}\left(F_{i}\right)} 1=\mathrm{c}-\operatorname{Ind}_{J}^{\mathrm{GL}_{m_{i}}(F)} \mathrm{c}-\operatorname{Ind}_{I}^{J} 1$. In Particular, the center of $H\left(\mathrm{GL}_{m_{i}}\left(F_{i}\right)\right.$, I) preserves the summand c-Ind $\mathrm{GL}_{J}\left(F_{i}\right) 1$; this gives a support-preserving map from the center of $H\left(\mathrm{GL}_{m_{i}}\left(F_{i}\right), I\right)$ to the spherical Hecke algebra $H\left(\mathrm{GL}_{m_{i}}\left(F_{i}\right)\right.$, $J)$. (This map simply gives the action of the center of the unipotent block on the induction $\mathrm{c}-\operatorname{Ind}_{J} \mathrm{GL}_{i}\left(F_{i}\right)$ 1.) One verifies easily that the action of the center of

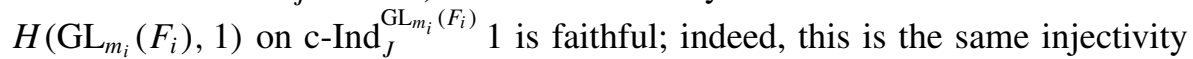
argument as in the proof of the injectivity of the map:

$$
Z\left(H\left(G, K_{P}, \mathrm{St}_{\bar{M}, s}\right)\right) \rightarrow H\left(G, K, \mathrm{St}_{s}\right) .
$$

On the other hand, the standard description of the action of the spherical Hecke algebra on the irreducible quotients of $\mathrm{c}-\mathrm{Ind}_{J} \mathrm{GL}_{m_{i}}\left(F_{i}\right) 1$, together with the Bernstein-Deligne description of the action of the center of $\operatorname{Rep}_{\overline{\mathcal{K}}}\left(\mathrm{GL}_{m_{i}}\left(F_{i}\right)\right)$ on these irreducibles, shows that for any element $x$ of the spherical Hecke algebra, there is an element of the center of $\operatorname{Rep}_{\overline{\mathcal{K}}}\left(\mathrm{GL}_{m_{i}}\left(F_{i}\right)\right)$ whose action on c-Ind $\mathrm{GL}_{m_{i}}\left(F_{i}\right) 1$ coincides with that of $x$. Since this element also gives an element of $Z\left(H\left(\mathrm{GL}_{m_{i}}\left(F_{i}\right), I\right)\right)$, we find that the map $Z\left(H\left(\mathrm{GL}_{m_{i}}\left(F_{i}\right), I\right)\right) \rightarrow H\left(\mathrm{GL}_{m_{i}}\left(F_{i}\right)\right.$, $J)$ is surjective, and hence an isomorphism.

Now the dimension of $H\left(\mathrm{GL}_{m_{i}}\left(F_{i}\right), J\right)_{J z J}$ is one for all $z$, so there is a onedimensional subspace of the center of $H\left(\mathrm{GL}_{m_{i}}\left(F_{i}\right), I\right)$ supported on $J z J$. Since $J z J$ is the union of $I w z w^{\prime} I$, as $w$ and $w^{\prime}$ range over elements of the Weyl group of $\mathrm{GL}_{m_{i}}\left(F_{i}\right)$, the remaining claims follow.

Our support-preserving isomorphism of $H\left(G, K_{P}, \mathrm{St}_{\bar{M}, s}\right)$ with the tensor product of the spaces $H\left(\mathrm{GL}_{m_{i}}\left(F_{i}\right), I\right)$ shows that for any $z$, there is a onedimensional subspace of $Z\left(H\left(G, K_{P}, \mathrm{St}_{\bar{M}, s}\right)\right.$ supported on the union of the double cosets of the form $K_{P} w z w^{\prime} K_{P}$, for $w$ and $w^{\prime}$ in $W_{\bar{M}}(s)$, and that the 
full center is the sum of these subspaces. Fix $z$, and let $S_{z}$ be the set of $z^{\prime} \in Z$ such that $K z^{\prime} K=K z K$. For any such $z^{\prime}$ we have $z^{\prime}=w^{-1} z w$ for some $w$ in the Weyl group of $G$. On the other hand, if $w z w^{-1}$ is an element $z^{\prime}$ of $Z$, then $w^{-1} M_{z} w=M_{z^{\prime}} \subseteq M$, so $w$ lies in $W\left(\bar{M}, \bar{M}_{z}\right)$. We thus have a surjection $W\left(\bar{M}, \bar{M}_{z}\right) \rightarrow S_{z}$, defined by $w \mapsto w^{-1} z w$. This surjection descends to a bijection of $W\left(\bar{M}_{z}\right) \backslash W\left(\bar{M}, \bar{M}_{z}\right)$ with $S_{z}$. Define an equivalence relation on $S_{z}$ by setting $z^{\prime} \sim z^{\prime \prime}$ if the collection of cosets $K_{P} w z^{\prime} w^{\prime} K_{P}\left(w, w^{\prime} \in W_{\bar{M}}(s)\right)$ coincides with the collection $K_{P} w z^{\prime \prime} w^{\prime} K_{P}\left(w, w^{\prime} \in W_{\bar{M}}(s)\right)$. Then $z^{\prime} \sim z^{\prime \prime}$ if, and only if, $K_{P} z^{\prime} K_{P}=K_{P} w z^{\prime \prime} w^{\prime} K_{P}$, for some $w$ and $w^{\prime}$ in $W_{\bar{M}}(s)$. This happens if, and only if, we have $z^{\prime}=w z^{\prime \prime} w^{-1}$ for some $w$ in $W_{\bar{M}}(s)$.

We thus obtain:

Proposition 6.11. The map $w \mapsto w^{-1} z w$ induces a bijection between: $i$

$$
W\left(\bar{M}_{z}\right) \backslash W\left(\bar{M}, \bar{M}_{z}\right) / W_{\bar{M}}(s) \rightarrow S_{z} / \sim \text {. }
$$

COROllary 6.12. The map:

$$
Z\left(H\left(G, K_{P}, \mathrm{St}_{\bar{M}, s}\right)\right) \rightarrow H\left(G, K, \mathrm{St}_{s}\right)
$$

is an isomorphism.

Proof. We have shown that this map is an injection, and that the dimension of the subspace of the target supported on $K z K$ is equal to the cardinality of $W\left(\bar{M}_{z}\right) \backslash W\left(\bar{M}, \bar{M}_{z}\right) / W_{\bar{M}}(s)$. On the other hand, for each equivalence class $\left[z^{\prime}\right]$ in $S_{z}$, we have a one-dimensional subspace of $Z\left(H\left(G, K_{P}, \mathrm{St}_{\bar{M}, s}\right)\right)$ supported on the union of cosets $K_{P} w z^{\prime} w^{\prime} K_{P}$; for distinct equivalence classes these spaces have disjoint supports contained in $K z K$. The dimension of $Z\left(H\left(G, K_{P}, \mathrm{St}_{\bar{M}, s}\right)\right)_{K z K}$ is therefore at least the cardinality of $S_{z}$, and the result is immediate from Proposition 6.11.

On the other hand, we have shown that the map $\operatorname{Hom}_{K_{P}}\left(\operatorname{St}_{\bar{M}_{, s}},-\right)$ is an equivalence of categories between $\operatorname{Rep}_{\overline{\mathcal{K}}}(G)_{M, \pi}$ and the category of $H\left(G, K_{P}, \mathrm{St}_{\bar{M}, s}\right)$-modules. It follows that the map $A_{M, \pi} \rightarrow H\left(G, K_{P}, \mathrm{St}_{\bar{M}, s}\right)$ giving the action of $A_{M, \pi}$ on $\mathrm{c}-\mathrm{Ind}_{K_{P}}^{G} \mathrm{St}_{\bar{M}, s}$ identifies $A_{M, \pi}$ with the center of $H\left(G, K_{P}, \mathrm{St}_{\bar{M}, s}\right)$. From this point of view, the corollary above asserts that the map $A_{M, \pi} \rightarrow H\left(G, K, \mathrm{St}_{s}\right)$ giving the action of $A_{M, \pi}$ on $\mathrm{c}-\mathrm{Ind}_{K}^{G} \mathrm{St}_{s}$ is an isomorphism.

\section{Endomorphisms of $\mathcal{P}_{K, \tau}$ in depth zero}

Our next objective is to refine the results of the previous section, in order to obtain results that hold over $W(k)$ rather than $\overline{\mathcal{K}}$. We retain the assumption that 
we are in depth zero, so that $K$ is a maximal compact and $\tau$ is inflated from a cuspidal representation of the $k$-points of a general linear group.

For our purposes it will be necessary to work inductively, with a sequence of cuspidal types. Fix an integer $n_{1}$ and let $\bar{G}_{1}$ be the group $\mathrm{GL}_{n_{1}}(k)$. If we fix a supercuspidal representation $\sigma_{1}$ of $\bar{G}_{1}$, corresponding to an irreducible $\ell$-regular conjugacy class $s_{1}^{\prime}$ in $\bar{G}_{1}$ then, for each $m$ in the set $\left\{1, e_{q}, \ell e_{q}, \ell^{2} e_{q}, \ldots\right\}$ (where $e_{q}$ is the order of $q \bmod \ell$ ) we have a cuspidal representation $\sigma_{m}$ of $\bar{G}_{m}:=\mathrm{GL}_{n_{1} m}(k)$ corresponding to the conjugacy class $\left(s_{1}^{\prime}\right)^{m}$ in $\bar{G}_{m}$.

For each $m$, we obtain a cuspidal type $\left(K_{m}, \tau_{m}\right)$ in $G_{m}:=\mathrm{GL}_{n_{1} m}(F)$ by setting $K_{m}=\mathrm{GL}_{n_{1} m}\left(\mathcal{O}_{F}\right)$ and taking $\tau_{m}$ to be the inflation of $\sigma_{m}$ to $K_{m}$. Then if we write $\mathcal{P}_{m}$ for the projective envelope of $\sigma_{m}$, we have $\mathcal{P}_{K_{m}, \tau_{m}}=\mathrm{c}-\operatorname{Ind}_{K_{m}}^{G_{m}} \mathcal{P}_{m}$.

Let $E_{m}$ denote the endomorphism ring of $\mathcal{P}_{K_{m}, \tau_{m}}$. The isomorphism:

$$
\mathcal{P}_{m} \otimes \overline{\mathcal{K}} \cong \bigoplus_{s} \mathrm{St}_{s}
$$

where $s$ runs over the conjugacy classes in $\bar{G}_{m}$ with $\ell$-regular part $\left(s_{1}^{\prime}\right)^{m}$, induces an isomorphism:

$$
\mathcal{P}_{K_{m}, \tau_{m}} \otimes \overline{\mathcal{K}} \cong \bigoplus_{s} \mathrm{c}-\operatorname{Ind}_{K_{m}}^{G_{m}} \mathrm{St}_{s}
$$

By the previous section, the summand c- $\operatorname{Ind}_{K_{m}}^{G_{m}} \operatorname{St}_{s}$ lies in $\operatorname{Rep}_{\overline{\mathcal{K}}}\left(G_{m}\right)_{M_{s}, \pi_{s}}$, and the pairs $\left(M_{s}, \pi_{s}\right)$ are not inertially equivalent for distinct $s$. Thus any endomorphism of $\mathcal{P}_{K_{m}, \tau_{m}}$ preserves each of the summands c-Ind ${ }_{K_{m}}^{G_{m}} \mathrm{St}_{s}$, and we have an isomorphism:

$$
E_{m} \otimes \overline{\mathcal{K}} \cong \prod_{s} A_{M_{s}, \pi_{s}}
$$

where for each $s, \pi_{s}$ is a supercuspidal representation of $M_{s}$ containing the type $\left(K_{M_{s}}, \mathrm{St}_{\bar{M}_{s}, s}\right)$.

We will inductively construct a family of endomorphisms $\Theta_{1, m}, \ldots, \Theta_{m, m}$ of $\mathcal{P}_{K_{m}, \tau_{m}}$. These endomorphisms will be characterized by their images in $A_{M_{s}, \pi_{s}}$ for each $s$. We describe the rings $A_{M_{s}, \pi_{s}}$ more concretely as follows: fix a uniformizer $\varpi$ of $F$, and let $Z_{s}$ denote the subgroup of $M_{s}$ consisting of elements of the center of $M_{s}$ whose characteristic polynomial has the form $(t-1)^{a}(t-\varpi)^{b}$ for integers $a$ and $b$. We then have a map $\overline{\mathcal{K}}\left[Z_{s}\right] \rightarrow \overline{\mathcal{K}}\left[M_{s} /\left(M_{s}\right)^{0}\right]$ that takes an element of $Z_{s}$ to its class modulo $\left(M_{s}\right)^{0}$, and its image is the subalgebra $\overline{\mathcal{K}}\left[M_{s} /\left(M_{s}\right)^{0}\right]^{H_{s}}$, where $H_{s}$ is the group of unramified characters $\chi$ of $M_{s}$ such that $\pi_{s} \otimes \chi$ is isomorphic to $\pi_{s}$.

A particular choice of $\pi_{s}$ gives rise to an isomorphism:

$$
A_{M_{s}, \pi_{s}} \cong\left(\overline{\mathcal{K}}\left[M_{s} /\left(M_{s}\right)^{0}\right]^{H_{s}}\right)^{W_{M_{s}}(s)}
$$


as in 3.5. It will therefore be important to have a systematic way of choosing the $\pi_{s}$. In depth zero this is rather straightforward: fix a uniformizer $\varpi$ of $F$. Then for a given $m$, and any irreducible conjugacy class $s$ in $\bar{G}_{m}$ with $\ell$-regular part $\left(s_{0}\right)^{m}$, we extend $\mathrm{St}_{s}$ to a representation $\Lambda_{s}$ of $F^{\times} K_{m}$ by letting $\varpi$ act via the identity. Then c-Ind ${ }_{F^{\times} K_{m}}^{G_{m}} \Lambda_{s}$ is an irreducible supercuspidal representation of $G_{m}$, which we denote by $\pi_{m, s}$. We call the collection $\left\{\pi_{m, s}\right\}$ the compatible family of cuspidals attached to $s_{1}^{\prime}$ and our choice of $\varpi$.

For an arbitrary $s$ with $\ell$-regular part $\left(s_{1}^{\prime}\right)^{m}$, we let $s_{1}, \ldots, s_{r}$ be the irreducible factors of $s$, of block sizes $n_{1} m_{1}, \ldots, n_{1} m_{r}$, and let $M_{s}$ be standard Levi of $\mathrm{GL}_{n_{0} m}(F)$ with block sizes $n_{1} m_{1}, \ldots, n_{1} m_{r}$, and take $\pi_{m, s}$ to be the tensor product of the $\pi_{m_{i}, s_{i}}$. This fixes, for all $s$, a corresponding isomorphism:

$$
A_{M_{s}, \pi_{s}} \cong\left(\overline{\mathcal{K}}\left[M_{s} /\left(M_{s}\right)^{0}\right]^{H_{s}}\right)^{W_{M_{s}}(s)} .
$$

We next define distinguished elements of $A_{M_{s}, \pi_{s}}$ :

DEFINITION 7.1. For $1 \leqslant i \leqslant m$, and $s$ a semisimple conjugacy class in $\bar{G}_{m}$ with $\ell$-regular part $\left(s_{1}^{\prime}\right)^{m}$, let $\theta_{i, s}$ be the image, in $\overline{\mathcal{K}}\left[M_{s} /\left(M_{s}\right)^{0}\right]$ of the sum of the elements $z$ in $Z_{s}$ with characteristic polynomial $(t-1)^{n_{1}(m-i)}(t-\varpi)^{n_{1} i}$. (Note that this sum may be empty, in which case $\theta_{i, s}=0$.)

The elements $\theta_{i, s}$ are clearly invariant under the actions of both $H_{s}$ and $W_{M_{s}}(s)$, and we may thus regard them as elements of $A_{M_{s}, \pi_{s}}$ via our chosen isomorphism. This allows us to give a characterization of the endomorphisms $\Theta_{i, m}$.

THEOREM 7.2. For $i \leqslant i \leqslant m$, there exists a unique endomorphism $\Theta_{i, m}$ in $E_{m}$ such that for all $s$ with $\ell$-regular part $\left(s_{1}^{\prime}\right)^{m}$, the composed map

$$
E_{m} \rightarrow E_{m} \otimes \overline{\mathcal{K}} \rightarrow A_{M_{s}, \pi_{s}}
$$

takes $\Theta_{i, m}$ to $\theta_{i, s}$. Moreover, $\Theta_{m, m}$ is invertible.

It is clear that such a $\Theta_{i, m}$ is unique if it exists; the construction of the $\Theta_{i, m}$ will occupy the remainder of the section.

The construction of $\Theta_{m, m}$ for any $m$ is straightforward: it is simply the endomorphism of c-Ind $\bar{K}_{m}^{G_{m}} \mathcal{P}_{m}$ given by the action of the central element $\varpi$ of $G_{m}$. This is clearly invertible, and its image in $A_{M_{s}, \pi_{s}}$ is equal to $\theta_{m, s}$.

The $\Theta_{i, m}$ for $i<m$ will be constructed by an inductive argument. Let $m^{\prime}<m$ be two consecutive elements of the set $\left\{1, e_{q}, \ell e_{q}, \ell^{2} e_{q}, \ldots\right\}$, and set $j=m / m^{\prime}$, so that $j=\ell$ or $j=e_{q}$. Let $\bar{M}$ be the standard Levi of $\bar{G}_{m}$ given by $j$ blocks of size $m^{\prime}$, and let $\bar{P}$ be the standard (upper triangular) parabolic with Levi $\bar{M}$. 
Further let $\mathcal{P}_{\bar{M}}$ be the representation of $\mathcal{P}_{m^{\prime}}^{\otimes j}$ of $\bar{M}$, and let $K^{\prime}$ be the preimage of $\bar{P}$ in $K_{m}$. Also let $M$ denote the standard Levi of $G_{m}$ given by $j$ blocks of size $m^{\prime}$ and let $K_{M}$ be the maximal compact subgroup $K^{\prime} \cap M$ of $M$. We may regard $\mathcal{P}_{\bar{M}}$ as a representation of $K^{\prime}$ or of $K_{M}$. The key point in our inductive argument is then:

THEOREM 7.3. The pair $\left(K^{\prime}, \mathcal{P}_{\bar{M}}\right)$ is a $G$-cover of $\left(K_{M}, \mathcal{P}_{\bar{M}}\right)$.

The proof of this is somewhat complicated and will be postponed to the end of the section. An immediate consequence is that we have a sequence of maps:

$$
E_{m^{\prime}}^{\otimes j} \cong H\left(M, K_{M}, \mathcal{P}_{\bar{M}}\right) \rightarrow H\left(G_{m}, K^{\prime}, \mathcal{P}_{\bar{M}}\right) \cong H\left(G_{m}, K_{m}, i \bar{G}_{\bar{P}} \mathcal{P}_{\bar{M}}\right)
$$

where $\bar{P}$ is the standard (block upper triangular) parabolic with Levi $\bar{M}$. Our first step is to compare the latter with $E_{m}$.

By Proposition 5.10, we have an isomorphism: $r_{\bar{G}_{m}}^{\bar{P}_{M}} \mathcal{P}_{m} \cong \mathcal{P}_{\bar{M}}$. Frobenius reciprocity then gives us a map:

$$
i \frac{\bar{G}_{m}}{P} \mathcal{P}_{\bar{M}} \rightarrow \mathcal{P}_{m} .
$$

Let $\mathcal{P}_{m}^{\prime}$ be the image of this map.

Lemma 7.4. The quotient $\mathcal{P}_{m} / \mathcal{P}_{m}^{\prime}$ is cuspidal. Moreover, every endomorphism of $\mathcal{P}_{m}$ preserves $\mathcal{P}_{m}^{\prime}$.

Proof. Note that the composition:

$$
\mathcal{P}_{\bar{M}} \rightarrow r \bar{G}_{m}^{\bar{P}} i \bar{G}_{m} \mathcal{P}_{\bar{M}} \rightarrow r_{\bar{G}_{m}}^{\bar{P}} \mathcal{P}_{m}
$$

is an isomorphism by construction. In particular, the map

$$
r_{\bar{G}_{m}}^{\bar{P}} i \bar{G}_{m} \mathcal{P}_{\bar{M}} \rightarrow r_{\bar{G}_{m}}^{\bar{P}} \mathcal{P}_{m}
$$

is surjective, and factors through $r_{\bar{G}_{m}}^{\bar{P}} \mathcal{P}_{m}^{\prime}$. The inclusion of $\mathcal{P}_{m}^{\prime}$ in $\mathcal{P}_{m}$ thus inducts an isomorphism:

$$
r_{\bar{G}_{m}}^{\bar{P}} \mathcal{P}_{m}^{\prime} \cong r_{\bar{G}_{m}}^{\bar{P}} \mathcal{P}_{m}
$$

Now let $\pi$ be a Jordan-Hölder constituent of $\mathcal{P}_{m} / \mathcal{P}_{m}^{\prime}$. Then $r_{\bar{G}_{m}}^{\bar{P}} \pi=0$ by the above calculation. Suppose $\pi$ were not cuspidal. Then it would have cuspidal support $\left(\bar{M}^{\prime}, \pi^{\prime}\right)$ for some proper Levi subgroup $\bar{M}^{\prime}$ of $\bar{G}_{m}$, with $\pi^{\prime}$ a product of cuspidal representations $\sigma_{m^{\prime \prime}}$, with $m^{\prime \prime}<m$. In particular, $\bar{M}^{\prime}$ would then be conjugate to a subgroup of $\bar{M}$, and $r_{\bar{G}_{m}}^{\bar{P}} \pi$ could not be trivial. 
Let $f$ be an endomorphism of $\mathcal{P}_{m}$, and suppose $f$ does not preserve $\mathcal{P}_{m}^{\prime}$. Then the image of $f\left(\mathcal{P}_{m}^{\prime}\right)$ in $\mathcal{P}_{m} / \mathcal{P}_{m}^{\prime}$ is nonzero, so we have a nonzero map from $\mathcal{P}_{m}^{\prime}$ to a cuspidal representation of $\overline{G_{m}}$. This induces a nonzero map of $i \overline{\bar{G}_{m}} \mathcal{P}_{\bar{M}}$ to a cuspidal representation of $\overline{G_{m}}$, which is impossible.

Our next step is to compare $H\left(G_{m}, K_{m}, \mathcal{P}_{m}\right)$ with $H\left(G_{m}, K_{m}, \mathcal{P}_{m}^{\prime}\right)$. Let $Z_{m}$ be the subgroup of $G_{m}$ consisting of diagonal matrices whose entries are powers of $\varpi$. Then $G_{m}$ is the union of double cosets $K_{m} z K_{m}$ for $z \in Z_{m}$. Moreover, for any $z$, we have isomorphisms:

$$
\begin{aligned}
& H\left(G_{m}, K_{m}, \mathcal{P}_{m}\right)_{K_{m} z K_{m}} \cong I_{z}\left(\mathcal{P}_{m}\right) \cong \operatorname{End}_{\bar{M}_{z}}\left(r_{\bar{G}_{m}}^{\bar{P}_{z}} \mathcal{P}_{m}\right) \\
& H\left(G_{m}, K_{m}, \mathcal{P}_{m}^{\prime}\right)_{K_{m} z K_{m}} \cong I_{z}\left(\mathcal{P}_{m}^{\prime}\right) \cong \operatorname{End}_{\bar{M}_{z}}\left(r_{\bar{G}_{m}} \overline{\mathcal{P}}_{m}^{\prime}\right)
\end{aligned}
$$

where $\bar{P}_{z}$ is the image in $\bar{G}_{m}$ of $K_{m} \cap z K_{m} z^{-1}$, and $\bar{M}_{z}$ is the associated Levi.

If $z$ is not central in $G_{m}$, then $\bar{M}_{z}$ is a proper subgroup of $\bar{G}_{m}$. Hence parabolic restriction to $\bar{M}_{z}$ annihilates the cokernel of the map $\mathcal{P}_{m}^{\prime} \rightarrow \mathcal{P}_{m}$, and so this inclusion induces an isomorphism of $r_{\bar{G}_{m}}^{\bar{P}_{z}} \mathcal{P}_{m}^{\prime}$ with $r_{\bar{G}_{m}}^{\bar{P}_{z}} \mathcal{P}_{m}$. By contrast, if $z$ is central, then $H\left(G_{m}, K_{m}, \mathcal{P}_{m}\right)_{K_{m} z K_{m}}$ is given by the endomorphism ring of $\mathcal{P}_{m}$, and hence, by Lemma 7.4 maps to the endomorphism ring of $\mathcal{P}_{m}^{\prime}$ (and thus to $\left.H\left(G_{m}, K_{m}, \mathcal{P}_{m}^{\prime}\right)\right)$. Combining these maps for all $z$ (central or otherwise) we find:

PROPOSITION 7.5. There is a surjection:

$$
H\left(G_{m}, K_{m}, \mathcal{P}_{m}\right) \rightarrow H\left(G_{m}, K_{m}, \mathcal{P}_{m}^{\prime}\right)
$$

compatible with the inclusion of $\mathrm{c}-\operatorname{Ind}_{K_{m}}^{G_{m}} \mathcal{P}_{m}^{\prime}$ in $\mathrm{c}-\operatorname{Ind}_{K_{m}}^{G_{m}} \mathcal{P}_{m}$ and the actions of the respective Hecke algebras on these spaces. Moreover, this map is an isomorphism away from double cosets of the form $K_{m} z K_{m}$ for z central in $G_{m}$.

Our next objective will be to compare the Hecke algebras $H\left(G_{m}, K_{m}, \mathcal{P}_{m}^{\prime}\right)$ and $H\left(G_{m}, K_{m}, i \bar{G}_{\bar{P}} \mathcal{P}_{\bar{M}}\right)$. By construction there is a surjection:

$$
i_{\bar{P}}^{\bar{G}_{m}} \mathcal{P}_{\bar{M}} \rightarrow \mathcal{P}_{m}^{\prime}
$$

and this surjection induces a surjection of c-Ind $K_{m}^{G_{m}} i \frac{\bar{G}_{m}}{P}$ onto c-Ind $K_{m}^{G_{m}} \mathcal{P}_{m}^{\prime}$. We then have:

LEMMA 7.6. Let $c$ be a central element of $H\left(G_{m}, K_{m}, i \frac{\bar{G}_{m}}{\bar{P}} \mathcal{P}_{\bar{M}}\right)$. Then c preserves the kernel of the map:

$$
\mathrm{c}-\operatorname{Ind}_{K_{m}}^{G_{m}} i \bar{G}_{\bar{P}} \rightarrow \mathrm{c}-\operatorname{Ind}_{K_{m}}^{G_{m}} \mathcal{P}_{m}^{\prime}
$$

and therefore descends to an element of $H\left(G_{m}, K_{m}, \mathcal{P}_{m}^{\prime}\right)$ with the same support as c. 
Proof. After inverting $\ell, \mathcal{P}_{m}^{\prime}$ becomes a direct summand of $i \bar{G}_{\bar{P}} \mathcal{P}_{\bar{M}}$, and so the module c-Ind ${ }_{K_{m}}^{G_{m}} \mathcal{P}_{m}^{\prime} \otimes \mathcal{K}$ is a direct summand of c-Ind ${ }_{K_{m}}^{G_{m}} i \bar{G}_{\bar{P}} \mathcal{P}_{\bar{M}} \otimes \mathcal{K}$. The element $c$ commutes with projection onto this summand, and so preserves the kernel of this projection. On the other hand, c-Ind $K_{m}^{G_{m}} \mathcal{P}_{m}^{\prime}$ is $\ell$-torsion free, and so the kernel of the map from c-Ind $K_{m}^{G_{m}} i \bar{G}_{\bar{P}} \mathcal{P}_{\bar{M}}$ onto c-Ind ${ }_{K_{m}}^{G_{m}} \mathcal{P}_{m}^{\prime}$ consists of those elements of the kernel of the projection that lie in c-Ind $K_{m}^{G_{m}} i \bar{G}_{\bar{P}} \mathcal{P}_{\bar{M}}$. If $x$ is such an element, it is clear that $c x$ is as well.

We can now sketch an inductive construction of the $\Theta_{i, m}$. Assume that for each $1 \leqslant i \leqslant m^{\prime}$, we have constructed elements $\Theta_{i, m^{\prime}}$ as in Theorem 7.2. Assume further that for $i<m^{\prime}$ the element $\Theta_{i, m^{\prime}}$ of $H\left(G_{m^{\prime}}, K_{m^{\prime}}, \mathcal{P}_{m^{\prime}}\right)$ is supported away from double cosets of the form $K_{m^{\prime}} z K_{m^{\prime}}$ for $z \in Z_{m^{\prime}}$ central. Then we can construct the $\Theta_{i, m}$ for $i<m^{\prime}$, as follows:

First, let $\tilde{\Theta}_{i, m}$ be the element of $E_{m^{\prime}}^{\otimes j}$ by the formula:

$$
\tilde{\Theta}_{i, m}=\sum_{r_{1}+\cdots+r_{j}=i} \Theta_{r_{1}, m^{\prime}} \otimes \cdots \otimes \Theta_{r_{j}, m^{\prime}}
$$

where $0 \leqslant r_{k} \leqslant i$ for all $k$ and by convention $\Theta_{0, m^{\prime}}=1$. For any $s_{1}, \ldots, s_{j}$, each with $\ell$-regular part $\left(s_{1}^{\prime}\right)^{m^{\prime}}$, the maps $E_{m^{\prime}} \rightarrow \overline{\mathcal{K}}\left[Z_{s_{k}}\right]$ takes $\Theta_{i, m^{\prime}}$ to $\theta_{i, s_{k}}$. Taking the tensor product of each of these maps gives, for $s$ the conjugacy class in $\bar{G}_{m}$ with 'blocks' $s_{k}$, a map:

$$
E_{m^{\prime}}^{\otimes j} \rightarrow \overline{\mathcal{K}}\left[Z_{s}\right]
$$

that takes $\tilde{\Theta}_{i, m}$ to $\theta_{i, s}$.

Let $\bar{\Theta}_{i, m}$ be the image of $\tilde{\Theta}_{i, m}$ under the map $E_{m^{\prime}}^{\otimes j} \rightarrow H\left(G_{m}, K_{m}^{\prime}, \mathcal{P}_{\bar{M}}\right)$ coming from Theorem 7.3. Note that, for $i<m$, the element $\bar{\Theta}_{i, m}$ is supported away from double cosets of the form $K_{m} z K_{m}$ with $z$ central in $G_{m}$. (This is because $\bar{\Theta}_{i, m}=u T^{+}\left(\tilde{\Theta}_{i, m}\right) u^{-1}$ for an invertible element $u$ of $H\left(G_{m}, K_{m}, i \overline{\bar{G}_{m}} \mathcal{P}_{\bar{M}}\right)$. Since for $z$ central, $z$ normalizes $K_{m}$, so conjugation by $u$ preserves $H\left(G_{m}, K_{m}, i \bar{G}_{\bar{P}}\right)_{K_{m} z K_{m}}$. Since $T^{+}\left(\tilde{\Theta}_{i, m}\right)$ has no support on $K_{m} z K_{m}$, neither can $\bar{\Theta}_{i, m}$.)

It now suffices to show that $\bar{\Theta}_{i, m}$ lies in the center of $H\left(G_{m}, K_{m}^{\prime}, \mathcal{P}_{\bar{M}}\right)$. Suppose this is the case. Then $\bar{\Theta}_{i, m}$ gives rise to an element of $H\left(G_{m}, K_{m}, \mathcal{P}_{m}^{\prime}\right)$ supported away from $K_{m} z K_{m}$ for $z$ central. There is thus a unique lift of the image of $\bar{\Theta}_{i, m}$ to an element $\Theta_{i, m}$ of $H\left(G_{m}, K_{m}, \mathcal{P}_{m}\right)$ supported away from $K_{m} z K_{m}$ for $z$ central.

Now let $s$ be a semisimple conjugacy class in $\bar{G}_{m}$ with $\ell$-regular part $\left(s^{\prime}\right)^{m}$. If $s$ is irreducible, then $\theta_{i, s}=0$ for $i<m$, and any element of $H\left(G_{m}, K_{m}, \mathcal{P}_{m}\right)$ supported away from the cosets $K_{m} z K_{m}$ for $z$ central maps to zero in $H\left(G_{m}, K_{m}, \mathrm{St}_{s}\right)$. Thus $\Theta_{i, m}$ maps to $\theta_{i, s}$ for such $s$. 
On the other hand, if $s$ is not irreducible, we may choose semisimple conjugacy classes $s_{1}, \ldots, s_{j}$ in $G_{m^{\prime}}$ such that $s$ is conjugate to the element of $G_{m}$ with 'blocks' $s_{1}, \ldots, s_{j}$, and let $\mathrm{St}_{\bar{M}, \vec{s}}$ denote the tensor product of the representations $\mathrm{St}_{s_{i}}$, considered as a representation of $\mathrm{St}_{\bar{M}}$. We have a map $i \bar{G}_{\bar{P}} \mathrm{St}_{\bar{M}, \vec{s}} \rightarrow \mathrm{St}_{s}$, and the action of $\Theta_{i, m}$ on c-Ind $K_{K_{m}}^{G_{m}} \mathrm{St}_{s}$ is compatible, via this map, with the action of $\bar{\Theta}_{i, m}$ on the summand c-Ind ${ }_{K_{m}}^{G_{m}} i \bar{G}_{\bar{P}} \mathrm{St}_{\bar{M}, \bar{s}}$ of c-Ind $K_{m}^{G_{m}} i \bar{G}_{\bar{P}} \mathcal{P}_{M} \otimes \overline{\mathcal{K}}$.

On the other hand, because the maps of Hecke algebras corresponding to $G$-covers are compatible with parabolic induction, the action of $\bar{\Theta}_{i, m}$ on the compact induction c-Ind $K_{m}^{G_{m}} i \bar{G}_{\bar{P}} \mathrm{St}_{\bar{M}, \vec{s}}$ is compatible with the action of $\tilde{\Theta}_{i, m}$ on c-Ind $K_{K_{M}}^{M} \mathrm{St}_{\bar{M}, \vec{s}}$, in the following sense: We have commutative diagram:

$$
\begin{gathered}
E_{m^{\prime}}^{\otimes j} \quad \rightarrow \quad \bigotimes_{k} H\left(G_{m^{\prime}}, K_{m^{\prime}}, \mathrm{St}_{s_{k}}\right) \\
\downarrow \\
\downarrow\left(G_{m}, K_{m}, i_{\bar{P}}^{\bar{G}_{m}} \mathcal{P}_{M^{\prime}}\right)
\end{gathered}
$$

Recall that we have a fixed identifications of $A_{M_{s}, \pi_{s}}$ with a subalgebra of $\overline{\mathcal{K}}\left[Z_{s}\right]$. Similarly we may identify the center of $\operatorname{Rep}_{\overline{\mathcal{K}}}\left(G_{m^{\prime}}\right)_{M_{s_{k}}, \pi_{s_{k}}}$ with a subalgebra of $\overline{\mathcal{K}}\left[Z_{s_{k}}\right]$, and $Z_{s}$ is isomorphic to the product of the $Z_{s_{k}}$. We then have a commutative diagram:

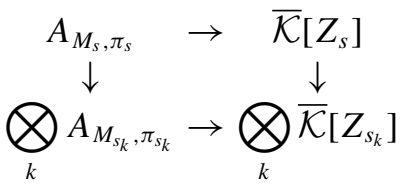

where the left-hand vertical map is given by Proposition 3.6.

The element $\theta_{i, s}$ of $\overline{\mathcal{K}}\left[Z_{s}\right]$ corresponds to an element of $A_{M_{s}, \pi_{s}}$ and this acts on c-Ind $K_{m}^{G_{m}} i \bar{G}_{\bar{P}} \mathrm{St}_{\bar{M}, \vec{s}}$; the above commutative diagram shows that this action is induced, via the map $T$, from the action of $\theta_{i, s}$ (considered as an element of $\bigotimes_{k} A_{M_{s_{k}}, \pi_{s_{k}}}$ ) on c-Ind $K_{M}^{M} \bigotimes_{k} \mathrm{St}_{s_{k}}$. Since the action of $\theta_{i, s}$ coincides with that of $\tilde{\Theta}_{i, m}$, and $T\left(\tilde{\Theta}_{i, m}\right)$ is equal to $\bar{\Theta}_{i, m}$ by definition, we conclude that the action of $\bar{\Theta}_{i, m}$ on c-Ind $K_{m}^{G_{m}} i \bar{G}_{\bar{P}} \operatorname{St}_{\bar{M}, \vec{s}}$ coincides with $\theta_{i, s}$. Together with the compatibility of $\bar{\Theta}_{i, m}$ and $\Theta_{i, m}$, this shows that $\Theta_{i, m}$ maps to $\theta_{i, s}$ for all $s$.

It thus remains to show that the element $\bar{\Theta}_{i, m}$ is central in $H\left(G_{m}, K_{m}^{\prime}, \mathcal{P}_{\bar{M}}\right)$. Since c-Ind ${ }_{K_{m}^{\prime}}^{G_{m}} \mathcal{P}_{\bar{M}}$ is $\ell$-torsion free, it suffices to prove this after tensoring with $\overline{\mathcal{K}}$. We will show that the action of $\bar{\Theta}_{i, m}$ coincides with the action of an element of the center of $\operatorname{Rep}_{\overline{\mathcal{K}}}\left(G_{m}\right)$, proving the claim. 
Indeed, let $x_{i}$ be an element of this center that acts via $\theta_{i, s}$ on $\operatorname{Rep}_{\overline{\mathcal{K}}}\left(G_{m}\right)_{M_{s}, \pi_{s}}$ for each semisimple conjugacy class $s$ with $\ell$-regular part $\left(s_{1}^{\prime}\right)^{m}$. Since the pairs $\left(M_{s}, \pi_{s}\right)$ give distinct blocks for distinct conjugacy classes $s$, such elements $x_{i}$ exist.

On the other hand, we have a direct sum decomposition:

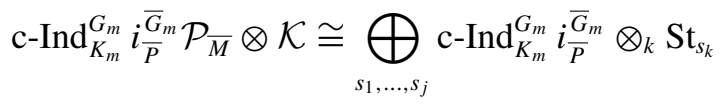

and (for $s$ the semisimple conjugacy class given by a block diagonal matrix whose blocks are the $s_{k}$ ) the action of $A_{M_{s}, \pi_{s}}$ on the summand corresponding to the $s_{k}$ is given by $\theta_{i, s}$. Thus the action of $\bar{\Theta}_{i, m}$ on c-Ind $\bar{K}_{M}^{G_{m}} i \bar{G}_{\bar{P}} \mathcal{P}_{\bar{M}}$ coincides with that of $x_{i}$, and is in particular central. Our construction of the $\Theta_{i, m}$ is thus complete, modulo the proof of Theorem 7.3, which will occupy the remainder of this section.

LEMMA 7.7. Let $P=M U$ be a parabolic subgroup of $G_{m}$ with Levi $M$, and let $(K, \tau)$ be a pair consisting of a compact open subgroup $K$ of $G_{m}$ and a $W(k)[K]$-module $\tau$ that is decomposed with respect to $P$. Set $K_{M}=K \cap M$, and let $\tau_{M}$ be the restriction of $\tau$ to $K_{M}$. Suppose that there are positive central elements $\lambda_{1}, \ldots, \lambda_{r}$ of $M$, whose product is strictly positive, and elements $x_{1}, \ldots, x_{r}$ of $H\left(M, K_{M}, \tau_{M}\right)$ such that for each $i, x_{i}$ is supported on $\lambda_{i} K_{M}$, and $T^{+} x_{i}$ is invertible in $H\left(G_{m}, K, \tau\right)$. Then $(K, \tau)$ is a $G_{m}$-cover of $\left(K_{M}, \tau_{M}\right)$.

Proof. Let $\lambda$ be the product of the $\lambda_{i}$, and $x$ the product of the $x_{i}$. Then $x$ is supported on $K_{m} \lambda K_{m}$, and $T^{+} x$ is the product of the $T^{+} x_{i}$, hence invertible. We must show that there is a strictly positive central element $\lambda^{\prime}$ of $M$ such that $1_{K_{m} \lambda^{\prime} K_{m}}$ maps, via $T^{+}$, to an invertible element of $H\left(G_{m}, K, \tau\right)$. Fix any strictly positive central $\lambda^{\prime}$ in $M$. Then for some sufficiently large $r, \lambda^{r}\left(\lambda^{\prime}\right)^{-1}$ is strictly positive. We have:

$$
T^{+}\left[\left(x^{r}\right) 1_{K_{m}\left(\lambda^{\prime}\right)^{-1} K_{m}}\right] T^{+}\left[1_{K_{m} \lambda^{\prime} K_{m}}\right]=T^{+} x^{r},
$$

and since $T^{+} x^{r}$ is invertible one must have $T^{+}\left[1_{K_{m} \lambda^{\prime} K_{m}}\right]$ invertible as well.

For $1 \leqslant i \leqslant j$, let $V_{i}$ be the span of the basis vectors $e_{m^{\prime}(i-1)+1}, \ldots, e_{m^{\prime} i}$ in $F^{n}$, so that $M$ is the Levi of $G_{m}$ preserving the $V_{i}$. Let $z$ be the central element of $M$ that acts by multiplication by $\varpi$ on $V_{1}$ and the identity on all other $V_{i}$, and let $w$ be the permutation matrix that maps $e_{i}$ to $e_{(j-1) m^{\prime}+i}$ for $1 \leqslant i \leqslant m^{\prime}$ and $e_{i}$ to $e_{i-m^{\prime}}$ for $m^{\prime}+1 \leqslant i \leqslant j m^{\prime}$. Set $\Pi=w z$. Then $\Pi$ normalizes $K^{\prime}$. Thus, if we let $\beta$ denote the automorphism of $\mathcal{P}_{\bar{M}}=\mathcal{P}_{m^{\prime}}^{\otimes j}$ that cyclically permutes the tensor factors, we have a unique element $y$ of $H\left(G_{m}, K^{\prime}, \mathcal{P}_{\bar{M}}\right)$ supported on $K^{\prime} \Pi K^{\prime}$ that takes the 
value $\beta$ at $\Pi$. Moreover, left multiplication by $y$ induces an isomorphism:

$$
H\left(G_{m}, K^{\prime}, \mathcal{P}_{M}\right)_{K^{\prime} x K^{\prime}} \rightarrow H\left(G_{m}, K^{\prime}, \mathcal{P}_{M}\right)_{K^{\prime} \Pi x K^{\prime}}
$$

for any $x$.

For $1 \leqslant i \leqslant j$, let $\lambda_{i}=w^{-i} \Pi^{i}$. Then $\lambda_{i}$ is a central, positive element of $M$ (but is not strictly positive). Let $x_{i}$ be the element of $H\left(M, K_{M}, \mathcal{P}_{\bar{M}}\right)$ giving the action of $\lambda_{i}$ on c-Ind $K_{M}^{M} \mathcal{P}_{\bar{M}}$. Then $x_{i}$ is supported on $K_{M} \lambda_{i} K_{M}$. It thus suffices to show that for each $i, T^{+} x_{i}$ invertible in $H\left(G_{m}, K^{\prime}, \mathcal{P}_{\bar{M}}\right)$.

Equivalently, it suffices to show that the element $v_{i}$ defined by $v_{i}=T^{+}\left(x_{i}\right) y^{-i}$ is invertible. Note that $v_{i}$ is supported on $K^{\prime} w^{-i} K^{\prime} \subset K$. It follows that the endomorphism $v_{i}$ of $\mathrm{c}-\operatorname{Ind}_{K^{\prime}}^{G_{m}} \mathcal{P}_{\bar{M}} \cong \mathrm{c}-\operatorname{Ind}_{K}^{G_{m}} i \bar{G}_{\bar{P}} \mathcal{P}_{\bar{M}}$ is induced by an endomorphism $\bar{v}_{i}$ of $i_{\bar{P}}^{\bar{G}_{m}} \mathcal{P}_{\bar{M}}$. We will show that $\bar{v}_{i}$ is an isomorphism.

Indeed, unwinding the definition of $v_{i}$, we see that when considered as an element of the Hecke algebra $H\left(\bar{G}_{m}, \bar{P}, \mathcal{P}_{\bar{M}}\right)$, the support of $\bar{v}_{i}$ is on the double coset $\bar{P} w^{-i} \bar{P}$, and $\bar{v}_{i}$ is the unique element supported on this double coset that is given at $w^{-i}$ by the isomorphism of $\mathcal{P}_{\bar{M}}$ with $\mathcal{P}_{\bar{M}}^{w^{-i}}$ that permutes the tensor factors.

Note that the map $\bar{U} \rightarrow\left(\bar{P} \cap w^{i} \bar{P} w^{-i}\right) \backslash \bar{P}$ is bijective. Thus the double coset $\bar{P} w^{-i} \bar{P}$ decomposes as the disjoint union of cosets $\bar{P} w^{-i} u$ as $u$ runs over the elements of $\bar{U}$. It follows that, if we regard elements of $i \frac{\bar{G}}{P} \mathcal{P}_{\bar{M}}$ as functions from $G$ to $\mathcal{P}_{\bar{M}}$, then the action of $\bar{v}_{i}$ on a function $f$ this space is given (up to a power of $p$ ) by first applying the 'permute the tensor factors' automorphism of $\mathcal{P}_{\bar{M}}$ to the values of $f$, and then summing over all the translates of this new function by elements of the form $w^{-i} u$, for $u \in \bar{U}$. This is precisely the map described in [HL, Theorem 2.4], where it is shown to be an isomorphism. Thus $v_{i}$ is an isomorphism for all $i$, completing the proof.

\section{Generic pseudotypes}

In this section and the next we will extend the techniques and arguments of the previous two sections to arbitrary generic pseudotypes. In every case the final results we obtain will be direct analogues of those from the depth zero case, but there are many more technicalities that need to be addressed.

Let $(K, \tau)$ be a maximal distinguished cuspidal $k$-type, with $\tau$ of the form $\kappa \otimes \sigma$. For suitable choice of $s$ we then obtain a generic pseudotype $\left(K, \tilde{\kappa} \otimes \mathrm{St}_{s}\right)$. Attached to the type $(K, \tau)$ is a finite extension $E$ of $F$, of ramification index $e$ and residue class degree $f$ over $F$, and an embedding $\operatorname{GL}_{n / e f}(E)$ into $G$ such $K$ contains $\mathrm{GL}_{n / e f}\left(\mathcal{O}_{E}\right)$ and the chain of maps:

$$
\mathrm{GL}_{n / e f}\left(\mathcal{O}_{E}\right) \rightarrow K \rightarrow K / K_{1}
$$

identifies $K / K_{1}$ with the quotient $\bar{G}=\mathrm{GL}_{n / e f}\left(\mathbb{F}_{q^{f}}\right)$ of $\mathrm{GL}_{n / e f}\left(\mathcal{O}_{E}\right)$. 
We will use these identifications to construct analogues of the Levi subgroups $M$ and $\bar{M}$ of Section 6. First, let $\bar{M}$ be the minimal split Levi of $\bar{G}$ containing $s$. We will make a choice of Levi subgroup $M$ of $G$ depending on $\bar{M}$, as follows: let $V$ be an $n$-dimensional $F$-vector space on which $G$ acts via the standard representation, so that $G \cong \mathrm{GL}(V)$. The distinguished subgroup $\mathrm{GL}_{n / e f}(E)$ of $G$ coming from the type $(K, \tau)$ gives $V$ the structure of an $E$-vector space. Let $L$ be an $\mathcal{O}_{E}$-lattice in $V$ stable under $\operatorname{GL}_{n / e f}\left(\mathcal{O}_{E}\right)$; then we have a map: $\mathrm{GL}_{n / e f}\left(\mathcal{O}_{E}\right) \rightarrow \bar{G}$ coming from the isomorphism: $\bar{G} \cong \mathrm{GL}_{\mathbb{F}_{q} f}\left(L / \varpi_{E} L\right)$. The Levi subgroup $\bar{M}$ of $\bar{G}$ then gives a direct sum decomposition:

$$
L / \varpi_{E} L=\oplus_{i} \bar{L}_{i},
$$

where the $\bar{L}_{i}$ are the minimal subspaces of $L / \varpi_{E} L$ stable under $\bar{M}$. Choose a lift of this to a direct sum decomposition:

$$
L=\oplus_{i} L_{i}
$$

of $\mathcal{O}_{E}$-modules, and hence a decomposition:

$$
V=\oplus_{i} V_{i}
$$

of $E$-vector spaces. Let $M$ be the corresponding Levi subgroup of $G$ (consisting of matrices that preserve each of the $V_{i}$ ). Then $M$ is a product of linear groups $M_{i}=\operatorname{Aut}_{F}\left(V_{i}\right)$. Note that the image of $M \cap \mathrm{GL}_{n / e f}\left(\mathcal{O}_{E}\right)$ in $\bar{G}$ is precisely $\bar{M}$.

Let $P$ be the parabolic subgroup of $G$ that preserves the subspaces $V_{1}, V_{1}+V_{2}$, and so forth, and let $U$ be its unipotent radical. Let $\bar{P}$ and $\bar{U}$ be the images of $P \cap \mathrm{GL}_{n / e f}\left(\mathcal{O}_{E}\right)$ and $U \cap \mathrm{GL}_{n / e f}\left(\mathcal{O}_{E}\right)$ in $\bar{G}$. Then $\bar{P}$ is a parabolic subgroup of $\bar{G}$ with Levi $\bar{M}$ and unipotent radical $\bar{U}$.

As in Section 6, the next step is to relate the pair $\left(K, \tilde{\kappa} \otimes \mathrm{St}_{s}\right)$ to a $G$-cover of a certain cuspidal $M$-type $\left(K_{M}, \tau_{M}\right)$. Our construction of the pair $\left(K_{M}, \tau_{M}\right)$ closely parallels [BK1, Sections 7.1 and 7.2], and is more or less the 'reverse' of the construction of [BK3, Section 7].

Recall that the maximal distinguished cuspidal type $(K, \tau)$ arises from a simple stratum $[\mathfrak{A}, n, 0, \beta]$, together with a character $\theta$ in $\mathcal{C}(\mathfrak{A}, 0, \beta)$. Given this data, the group $K$ is the group $J(\beta, \mathfrak{A})$ in [BK1], and the representation $\tilde{\kappa}$ of $K$ is a $\beta$-extension of the unique irreducible representation of $J^{1}(\beta, \mathfrak{A})$ whose restriction to the subgroup $H^{1}(\beta, \mathfrak{A})$ contains the character $\theta$.

Let $\mathfrak{A}_{i}$ be order in $\operatorname{End}_{F}\left(V_{i}\right)$ induced by $\mathfrak{A}$; that is, the image of the subring of $\mathfrak{A}$ that preserves $V_{i}$ in $\operatorname{End}_{F}\left(V_{i}\right)$. Then conjugation by $E^{\times}$stabilizes $\mathfrak{A}_{i}$ and $\mathfrak{A}_{i} \cap \operatorname{End}_{E}\left(V_{i}\right)=\operatorname{End}_{\mathcal{O}_{E}}\left(L_{i}\right)$. Given these orders, the procedure at the beginning of [BK3, 7.2] constructs an order $\mathfrak{A}^{\prime}$ in $\operatorname{End}_{F}(V)$ (this is the order denoted by $\mathfrak{A}$ 
in $\left[\mathbf{B K} 3\right.$, Section 7]). The order $\mathfrak{A}^{\prime}$ is contained in the maximal order $\mathfrak{A}$. Set $K^{\prime}=J\left(\beta, \mathfrak{A}^{\prime}\right)$.

Let $K_{P}$ be the preimage of $\bar{P}$ in $K$, via our identification of $K / K_{1}$ with $\bar{G}$. Then by [BK1, Theorem 5.2.3(ii)], there is a unique $W(k)\left[K^{\prime}\right]$-module $\tilde{\kappa}^{\prime}$ of $K^{\prime}$ such that we have:

$$
\left.\operatorname{Ind}_{K_{P}}^{\left(\mathfrak{A}^{\prime}\right)^{\times}} \tilde{\kappa}\right|_{K_{P}} \cong \operatorname{Ind}_{K^{\prime}}^{\left(\mathfrak{A}^{\prime}\right)^{\times}} \tilde{\kappa}^{\prime} .
$$

(Strictly speaking, this is proved in [BK1] over an algebraically closed field of characteristic zero, rather than over $W(k)$; in the proof of [V1, III.4.21] Vigneras observes that the same result holds for the $W(k)$-representations we use here.) Over $\mathcal{K}$, the representation $\tilde{\kappa}^{\prime}$ can alternatively be described, up to twist, in the following way: the character $\theta$ gives rise to an endo-class of ps-characters $(\Theta, 0$, $\beta)$ in the sense of [BK3, Section 4]. Then $(\Theta, 0, \beta)$ in particular give rise to a character $\theta^{\prime}$ in $\mathcal{C}\left(\mathfrak{A}^{\prime}, 0, \beta\right)$. From this perspective $\tilde{\kappa}^{\prime}$ is a $\beta$-extension of the unique irreducible representation of $J^{1}\left(\beta, \mathfrak{A}^{\prime}\right)$ whose restriction to $H^{1}\left(\beta, \mathfrak{A}^{\prime}\right)$ contains the character $\theta^{\prime}$.

We now apply the construction of $[\mathbf{B K} 1,7.2]$ to the representation $\tilde{\kappa}^{\prime}$. That is, let $K^{\prime \prime}$ be the subset $\left(J\left(\beta, \mathfrak{A}^{\prime}\right) \cap P\right) H^{1}\left(\beta, \mathfrak{A}^{\prime}\right)$; it is shown in [BK1, 7.1 and 7.2] that $K^{\prime \prime}$ is a group, and that the $K^{\prime} \cap U$-fixed vectors in $\tilde{\kappa}^{\prime}$ are stable under $K^{\prime \prime}$. Thus these fixed vectors give a representation $\tilde{\kappa}^{\prime \prime}$ of $K^{\prime \prime}$. Let $K_{M}$ be the intersection $K^{\prime \prime} \cap M$, and let $\tilde{\kappa}_{M}$ be the restriction of $\tilde{\kappa}^{\prime \prime}$ to $K_{M}$. We then have the following:

LEMMA 8.1. The pair $\left(K^{\prime \prime}, \tilde{\kappa}^{\prime \prime}\right)$ satisfies the conditions [BK3, (7.2.1)]. Explicitly:

(1) The restriction of $\tilde{\kappa}^{\prime \prime}$ to $H^{1}\left(\beta, \mathfrak{A}^{\prime}\right)$ is a multiple of $\theta^{\prime}$.

(2) The representation $\tilde{\kappa}^{\prime \prime}$ is trivial on $K^{\prime \prime} \cap U$ and $K^{\prime \prime} \cap U^{\circ}$.

(3) The group $K_{M}$ is the product of the groups $K_{i}=K \cap M_{i}$, and the representation $\tilde{\kappa}_{M}$ is a tensor product of irreducible representations $\tilde{\kappa}_{i}$ of $K_{i}$ for each $i$. Moreover, the subgroup $K_{i}$ of $M_{i}$ is equal to $J\left(\beta, \mathfrak{A}_{i}\right)$, and each $\tilde{\kappa}_{i}$ is a $\beta$-extension of the unique representation of $J^{1}\left(\beta, \mathfrak{A}_{i}\right)$ whose restriction to $H^{1}\left(\beta, \mathfrak{A}_{i}\right)$ contains the element $\theta_{i}$ of $\mathcal{C}\left(\mathfrak{A}_{i}, 0, \beta\right)$ determined by the ps-character $(\Theta, 0, \beta)$.

Moreover, the map:

$$
\operatorname{Ind}_{K^{\prime \prime}}^{K^{\prime}} \tilde{\kappa}^{\prime \prime} \rightarrow \tilde{\kappa}^{\prime}
$$

(obtained by Frobenius reciprocity from the realization of $\tilde{\kappa}^{\prime \prime}$ as the $U \cap K^{\prime \prime}$ invariants of $\left.\tilde{\kappa}^{\prime}\right)$ is an isomorphism.

Proof. The first claim follows from [BK1, 5.1.1] and our description of $\tilde{\kappa}^{\prime \prime}$ as a $\beta$-extension. The second is clear from the construction of $\tilde{\kappa}^{\prime \prime}$. The decomposition 
of $\tilde{\kappa}$ as a tensor product of $\tilde{\kappa}_{i}$ is [BK1, 7.2.14], as is the fact that each $\tilde{\kappa}_{i}$ is a $\beta$-extension (the necessary intertwining property on the $\tilde{\kappa}_{i}$ is verified as part of [BK1, 7.2.15]). The fact that the characters $\theta_{i}$ are the ones claimed in the theorem follows from [BK1, 7.1.19]. The isomorphism is [BK1, 7.2.15].

Let $s_{i}$ be the projection of $s$ to $\bar{M}_{i}$; then $s_{i}$ is a semisimple element of $\bar{M}_{i}$ with irreducible characteristic polynomial. As $K_{i}$ contains $J^{1}\left(\beta, \mathfrak{A}_{i}\right)$ as a normal subgroup, and the quotient is naturally isomorphic to $\bar{M}_{i}$, we may regard the cuspidal representation $\mathrm{St}_{s_{i}}$ of $\bar{M}_{i}$ as a representation of $K_{i}$. We set $\tau_{i}=\tilde{\kappa}_{i} \otimes \mathrm{St}_{s_{i}}$; the pair $\left(K_{i}, \tau_{i}\right)$ is then a maximal distinguished cuspidal type in $M_{i}$. Let $\tau_{M}$ be the tensor product of the $\tau_{i}$; it is then a representation of $K_{M}$, and we have $\tau_{M}=\tilde{\kappa}_{M} \otimes \mathrm{St}_{\bar{M}, s}$, where $\mathrm{St}_{\bar{M}, s}$ is the tensor product of the cuspidal representations $\mathrm{St}_{s_{i}}$. The pair $\left(K_{M}, \tau_{M}\right)$ is a maximal distinguished cuspidal $M$-type.

On the other hand, by construction, the quotient of $K^{\prime \prime}$ by $J^{1}\left(\beta, \mathfrak{A}^{\prime}\right)$ is naturally isomorphic to $\bar{M}$. We can thus regard $\mathrm{St}_{\bar{M}, s}$ as a representation of $K^{\prime \prime}$, and form the representation $\tau^{\prime \prime}=\tilde{\kappa}^{\prime \prime} \otimes \mathrm{St}_{\bar{M}, s}$. We will show that $\left(K^{\prime \prime}, \tau^{\prime \prime}\right)$ is a $G$-cover of $\left(K_{M}, \tau_{M}\right)$.

Note, however, that unlike in the level zero case, there is further work to be done: the pair $\left(K^{\prime \prime}, \tau^{\prime \prime}\right)$ must be related to the original pair $(K, \tau)$. To this end, consider the representation $\tau_{P}=\left.\tilde{\kappa}\right|_{K_{P}} \otimes \mathrm{St}_{\bar{M}, s}$, where we regard $\mathrm{St}_{\bar{M}, s}$ as a representation of $K_{P}$ via the surjection

$$
K_{P} \rightarrow \bar{P} \rightarrow \bar{M}
$$

We then have:

THEOREM 8.2. The pair $\left(K^{\prime \prime}, \tau^{\prime \prime}\right)$ is a $G$-cover of $\left(K_{M}, \tau_{M}\right)$. Moreover, there are natural isomorphisms:

$$
\begin{gathered}
\mathrm{c}-\operatorname{Ind}_{K^{\prime \prime}}^{\left(\mathfrak{A}^{\prime}\right)^{\times}} \tau^{\prime \prime} \cong \mathrm{c}-\operatorname{Ind}_{K_{P}}^{\left(\mathfrak{A}^{\prime}\right)^{\times}} \tau_{P} \\
\mathrm{c}-\operatorname{Ind}_{K^{\prime \prime}}^{\mathfrak{A} \times} \tau^{\prime \prime} \cong \mathrm{c}-\operatorname{Ind}_{K}^{\mathfrak{\mathfrak { A } ^ { \times }}} \tilde{\kappa} \otimes I_{s}
\end{gathered}
$$

where we regard $I_{s}$ as a representation of $K$ via the surjection of $K$ onto $\bar{G}$.

Proof. We have verified that $\left(K^{\prime \prime}, \tilde{\kappa}^{\prime \prime}\right)$ satisfies the list of properties in [BK3, 7.2.1]. In particular, if one applies the procedure of [BK3, Section 7.2] to the type $\left(K_{M}, \tau_{M}\right)$, one arrives at the representation $\left(K^{\prime \prime}, \tau^{\prime \prime}\right)$. Thus $\left(K^{\prime \prime}, \tau^{\prime \prime}\right)$ is a $G$-cover of $\left(K_{M}, \tau_{M}\right)$ by [BK3, Theorem 7.2]. (Notice that the procedure given there in particular applies to the type $\left(K_{M}, \tau_{M}\right)$ because we have verified that each of the types $\left(K_{i}, \tau_{i}\right)$ arises from the same endo-class $(\Theta, 0, \beta)$ of ps-character.) 
Whenever we have $H^{\prime}$ a subgroup of $H$, and representations $A$ of $H$ and $A^{\prime}$ of $H^{\prime}$, we have a general identity:

$$
\left.\operatorname{Ind}_{H^{\prime}}^{H} A\right|_{H^{\prime}} \otimes B \cong A \otimes \operatorname{Ind}_{H}^{H^{\prime}} B .
$$

It follows from this and the isomorphism:

$$
\tilde{\kappa}^{\prime} \cong \operatorname{Ind}_{K^{\prime \prime}}^{K^{\prime}} \tilde{\kappa}^{\prime}
$$

that we have an isomorphism:

$$
\mathrm{c}-\operatorname{Ind}_{K^{\prime \prime}}^{\mathfrak{A} \times} \tau^{\prime \prime} \cong \mathrm{c}-\operatorname{Ind}_{K^{\prime}}^{\mathfrak{A}^{\times}} \tau^{\prime},
$$

where $\tau^{\prime}=\tilde{\kappa}^{\prime} \otimes \mathrm{St}_{\bar{M}, s}$. (Recall that the surjections of $K^{\prime}$ and $K^{\prime \prime}$ onto $\bar{M}$ are compatible with the inclusion of $K^{\prime \prime}$ in $K^{\prime}$, so we can regard $\mathrm{St}_{\bar{M}, s}$ as a representation of $K^{\prime}$ here.)

Next, the isomorphism:

$$
\left.\operatorname{Ind}_{K^{\prime}}^{\left(\mathfrak{A}^{\prime}\right)^{\times}} \kappa^{\prime} \cong \operatorname{Ind}_{K_{P}}^{\left(\mathfrak{A}^{\prime}\right)^{\times}} \kappa\right|_{K_{P}}
$$

induces an isomorphism:

$$
\operatorname{Ind}_{K^{\prime}}^{\left(\mathfrak{A}^{\prime}\right)^{\times}} \tau^{\prime} \cong \operatorname{Ind}_{K_{P}}^{\left(\mathfrak{A}^{\prime}\right)^{\times}} \tau_{P} .
$$

Finally, we have an isomorphism:

$$
\left.\operatorname{Ind}_{K_{P}}^{\mathfrak{A} \times} \kappa\right|_{K_{P}} \otimes \mathrm{St}_{\bar{M}, s} \cong \operatorname{Ind}_{K}^{\mathfrak{A} \times} \kappa \otimes I_{s}
$$

obtained by inducing the previous isomorphism from $\left(\mathfrak{A}^{\prime}\right)^{\times}$to $\mathfrak{A}^{\times}$and applying the tensor product identity.

As in Corollary 6.6, the maximal distinguished cuspidal type $\left(K_{M}, \tau_{M}\right)$ gives rise to a unique inertial equivalence class of cuspidal representations of $M$; let $\pi$ be an irreducible representation of $M$ over $\overline{\mathcal{K}}$ that lies in this inertial equivalence class (or equivalently, that contains the type $\left(K_{M}, \tau_{M}\right)$ ). We then have:

COROLLARY 8.3. The $\overline{\mathcal{K}}[G]$-modules $\mathrm{c}-\operatorname{Ind}_{K}^{G} \tilde{\kappa} \otimes I_{s}$ and $\mathrm{c}-\operatorname{Ind}_{K}^{G} \tilde{\kappa} \otimes \mathrm{St}_{s}$ are objects of $\operatorname{Rep}_{\overline{\mathcal{K}}}(G)_{(M, \pi)}$.

Proof. Theorem 8.2 implies that we have an isomorphism:

$$
\mathrm{c}-\operatorname{Ind}_{K}^{G} \tilde{\kappa} \otimes I_{s} \cong \mathrm{c}-\operatorname{Ind}_{K^{\prime \prime}}^{G} \tau^{\prime \prime} .
$$

As $\left(K^{\prime \prime}, \tau^{\prime \prime}\right)$ is a $G$-cover of the maximal distinguished cuspidal type $\left(K_{M}, \tau_{M}\right)$, it follows immediately from [BK2, Theorem 8.3] that c-Ind $K^{\prime \prime} \tau^{\prime \prime}$ lies in the block 
$\operatorname{Rep}_{\bar{K}}(G)_{(M, \pi)}$. As for c-Ind ${ }_{K}^{G} \tilde{\kappa} \otimes \mathrm{St}_{s}$, note that, by definition, $\mathrm{St}_{s}$ is the (unique) generic summand of $I_{s}$, and so this $\overline{\mathcal{K}}[G]$-module is a direct summand of the first.

We now turn to the question of understanding the Hecke algebra $H(G, K$, $\left.\tilde{\kappa} \otimes \mathrm{St}_{s}\right)$, or equivalently the endomorphism ring $\operatorname{End}_{\overline{\mathcal{K}}[G]}\left(\mathrm{c}-\operatorname{Ind}_{K}^{G} \tilde{\kappa} \otimes \mathrm{St}_{s}\right)$. The isomorphisms of Theorem 8.2 induce isomorphisms:

$$
\begin{aligned}
H\left(G, K^{\prime \prime}, \tau^{\prime \prime}\right) \cong H\left(G,\left(\mathfrak{A}^{\prime}\right)^{\times}, \operatorname{Ind}_{K^{\prime \prime}}^{\left(\mathfrak{A}^{\prime} \times\right.} \tau^{\prime \prime}\right) \\
H\left(G, K_{P}, \tau_{P}\right) \cong H\left(G,\left(\mathfrak{A}^{\prime}\right)^{\times}, \operatorname{Ind}_{K^{\prime \prime}}^{\left(\mathfrak{A}^{\prime}\right)^{\times}} \tau^{\prime \prime}\right) .
\end{aligned}
$$

These isomorphisms are compatible with support in the sense that an element of one of the left-hand Hecke algebras supported on a double coset $K^{\prime \prime} g K^{\prime \prime}$ or $K_{P} g K_{P}$ gets sent to an element of the right-hand Hecke algebra supported on $\left(\mathfrak{A}^{\prime}\right)^{\times} g\left(\mathfrak{A}^{\prime}\right)^{\times}$.

This allows us to compare $H\left(G, K^{\prime \prime}, \tau^{\prime \prime}\right)_{K^{\prime \prime} g K^{\prime \prime}}$ and $H\left(G, K_{P}, \tau_{P}\right)_{K_{P} g K_{P}}$ for various $g$ in $G$.

On the one hand, the space $H\left(G, K^{\prime \prime}, \tau^{\prime \prime}\right)$ is well understood; just as in the depth zero case, the discussion in [BK3, Section 1] shows that it is a tensor product of affine Hecke algebras. As in the depth zero case, there is a sense in which the isomorphism to a tensor product of affine Hecke algebras is compatible with support, but (as we are no longer able to work with standard Levi and parabolic subgroups) it will take somewhat more work to make this precise. To begin with, recall that $M$ is the subgroup of $G$ consisting of endomorphisms of $V$ that preserve each summand $V_{i}$ of $V$, and let $Z$ be the subgroup of $M$ consisting of elements that act by a power of $\varpi_{E}$ on each $V_{i}$.

Choose a maximal torus $T_{E}$ of $\operatorname{GL}_{n / e f}(E)$, and let $\bar{T}$ be its reduction $\bmod \varpi_{E}$. Then $\bar{T}$ is a maximal torus of $\bar{G}$; we assume it is contained in $\bar{M}$. Let $W(\bar{G})$ be the Weyl group of $\bar{G}$ with respect to $\bar{T}$. The choices of $T_{E}$ and $\bar{T}$ give an isomorphism of $W(\bar{G})$ with the Weyl group of $\mathrm{GL}_{n / e f}(E)$.

Now choose a maximal torus $T_{F}$ of $G$. We will say that $T_{F}$ is compatible with $T_{E}$ if every $T_{E}$-stable $E$-line in $F^{n}$ is a sum of $T_{F}$-stable $F$-lines. A choice of $T_{F}$ compatible with $T_{E}$ identifies the Weyl group of $\mathrm{GL}_{n / e f}(E)$ with a subgroup of $W(G)$, and thus lets us consider $W(\bar{G})$ as a subgroup of $W(G)$. In what follows, whenever we have a trio of groups $G, \operatorname{GL}_{n / e f}(E), \bar{G}$, we will choose maximal tori of these groups related in the sense described above, and implicitly make the corresponding identifications on Weyl groups.

Let $W_{\bar{M}}$ be the subgroup of $W(\bar{G})$ normalizing $\bar{M}$, and let $W_{\bar{M}}(s)$ be the subgroup of $W_{\bar{M}}$ consisting of $w$ such that $w s w^{-1}$ is $M$-conjugate to $s$. If $W_{M}$ is the subgroup of $W(G)$ normalizing $M$, we can identify $W_{\bar{M}}$ with a subgroup of $W_{M}$. Then $H\left(G, K^{\prime \prime}, \tau^{\prime \prime}\right)$ is supported on the double cosets $K^{\prime \prime} g K^{\prime \prime}$ for $g$ 
in $W_{\bar{M}}(s) Z$, and each $H\left(G, K^{\prime \prime}, \tau^{\prime \prime}\right)_{K^{\prime \prime} g K^{\prime \prime}}$ is a one-dimensional $\overline{\mathcal{K}}$-vector space. (Observe that if $w, w^{\prime}$ are in $W_{\bar{M}}(s)$, and $z, z^{\prime}$ lie in $Z$, then $K^{\prime \prime} w z K^{\prime \prime}=K^{\prime \prime} w^{\prime} z^{\prime} K^{\prime \prime}$ if, and only if, $z=z^{\prime}$ and $w^{-1} w^{\prime}$ lies in $W(M)$.)

We now decompose $Z$ into a product of factors $Z_{j}$, just as we did in the depth zero setting. Specifically, if we write the characteristic polynomial of $s$ as a product of irreducible polynomials $f_{1}^{m_{1}} \ldots f_{r}^{m_{r}}$, with $\operatorname{deg} f_{j}=d_{j}$, then the quotient of $W_{\bar{M}}(s)$ by the subgroup $W(M)$ of $W_{\bar{M}}(s)$ is a product of permutation groups $W_{j} \cong S_{m_{j}}$, where $W_{j}$ permutes the 'blocks' of $s$ with characteristic polynomial $f_{j}$. If we let $Z_{j}$ be the subgroup of $Z$ consisting of elements that are the identity away from those blocks, then $Z_{j}$ is a subgroup of $Z$ invariant under the conjugation action of $W_{\bar{M}}(s)$, and the conjugation action of $W_{\bar{M}}(s)$ on $Z_{j}$ factors through $S_{m_{j}}$. Moreover, $W_{j} Z_{j}$ is a subgroup of $\mathrm{GL}_{n}$, and the subspace $H_{j}$ of $H(G$, $\left.K^{\prime \prime}, \tau^{\prime \prime}\right)$ supported on cosets of the form $K g K$ for $g$ in $W_{j} Z_{j}$ is a subalgebra of $H\left(G, K^{\prime \prime}, \tau^{\prime \prime}\right)$ isomorphic to the affine Hecke algebra $H\left(\mathrm{GL}_{n}\left(E_{j}\right), I\right)$, where $E_{j}$ is the unramified extension of $E$ of degree $d_{j}$, and $I$ is the Iwahori subgroup. (This isomorphism depends on certain choices and is therefore not canonical; we refer the reader to [BK1, 5.6], for its construction.)

The algebra $H\left(G, K^{\prime \prime}, \tau^{\prime \prime}\right)$ is then the tensor product of the $H_{j}$. Moreover, the map from $H\left(\mathrm{GL}_{n}\left(E_{j}\right), I\right)$ to $H_{j}$ is compatible with supports in a manner exactly analogous to the depth zero case. We may embed $\mathrm{GL}_{m_{j}}\left(E_{j}^{\prime}\right)$ in $M_{j}$ in such a way that the image of $\mathrm{GL}_{m_{j}}\left(\mathcal{O}_{E_{j}}\right)$ is equal to the intersection of $\mathfrak{A}$ with the image of $\mathrm{GL}_{m_{j}}\left(E_{j}\right)$, and so that the maximal tori of $M_{j}$ and $M_{j} \cap \mathrm{GL}_{n / e f}\left(E_{j}\right)$ arising from $T_{F}$ and $T_{E}$ are compatible with the standard maximal torus of $\mathrm{GL}_{m_{j}}\left(E_{j}\right)$. Then the reduction $\bmod \varpi_{E}$ of $\mathrm{GL}_{m_{j}}\left(\mathcal{O}_{E_{j}}\right)$ is a subgroup of $\bar{M}_{j}$ isomorphic to $\mathrm{GL}_{m_{j}}\left(\mathbb{F}_{q}{ }_{q d_{j}}\right)$; we assume we have chosen our embedding so that the standard maximal torus of $\mathrm{GL}_{m_{j}}\left(\mathbb{F}_{q^{f d_{j}}}\right)$ is contained in the Levi $\bar{M}_{s_{j}}$.

This embedding allows us to identify $W_{j}$ with the (standard) Weyl group $W_{j}^{\prime}$ of $\mathrm{GL}_{m_{j}}\left(E_{j}\right)$. Our choices identify $Z_{j}$ with a subgroup $Z_{j}^{\prime}$ of the diagonal matrices in $\mathrm{GL}_{m_{j}}\left(E_{j}\right)$, and then $\mathrm{GL}_{m_{j}}\left(E_{j}\right)$ is a union of double cosets $I_{j} w^{\prime} z^{\prime} I_{j}$, with $w^{\prime}$ in $W_{j}^{\prime}$ and $z^{\prime}$ in $Z_{j}^{\prime}$. The identification of $H\left(\mathrm{GL}_{m_{j}}\left(E_{j}\right), I\right)$ with $H_{j}$ then takes the subspace $H\left(\mathrm{GL}_{m_{j}}\left(E_{j}\right), I\right)_{I w^{\prime} z^{\prime} I}$ to $H\left(G, K^{\prime \prime}, \tau^{\prime \prime}\right)_{K^{\prime \prime} w z K^{\prime \prime}}$, where $w$ and $z$ are the elements of $W_{j}$ and $Z_{j}$ corresponding to $w^{\prime}$ and $z^{\prime}$.

Our identification of $H\left(G, K^{\prime \prime}, \tau^{\prime \prime}\right)$ with a tensor product of Iwahori Hecke algebras allows us to establish an 'integral' version of the fact that $\left(K^{\prime \prime}, \tau^{\prime \prime}\right)$ is a $G$-cover of $\left(K_{M}, \tau_{M}\right)$. More precisely, we have:

LEMmA 8.4. Let $L$ be a $W(k)$-lattice in $\mathrm{St}_{\bar{M}, s}$, and let $L_{M}$ be the lattice $\tilde{\kappa}_{M} \otimes L$ in $\tau_{M}$. Similarly, let $L^{\prime \prime}$ be the lattice $\tilde{\kappa}^{\prime \prime} \otimes L$ in $\tau^{\prime \prime}$. Then $\left(K^{\prime \prime}, L^{\prime \prime}\right)$ is a $G$-cover of $\left(K_{M}, L_{M}\right)$. Moreover, if $H_{j}^{\circ}$ denotes the intersection of $H_{j}$ with $H\left(K^{\prime \prime}, L^{\prime \prime}\right)$, 
and $H^{\circ}\left(\mathrm{GL}_{m_{j}}\left(E_{j}\right), I\right)$ is the Iwahori Hecke algebra over $W(k)$, then there is an isomorphism of $H_{j}$ with $H\left(\mathrm{GL}_{m_{j}}\left(E_{j}\right), I\right)$ that restricts to an isomorphism of $H_{j}^{\circ}$ with $H^{\circ}\left(\mathrm{GL}_{m_{j}}\left(E_{j}\right), I\right)$.

Proof. This is closely related to the arguments of [V2, IV.2.5 and IV.2.6].

It is clear that the only condition that needs to be verified is that there is an element of $H\left(K_{M}, L_{M}\right)$ with totally positive support whose image in $H\left(K^{\prime \prime}\right.$, $\left.L^{\prime \prime}\right)$ is invertible. This follows immediately if there is an isomorphism of $H_{j}^{\circ}$ with $H^{\circ}\left(\mathrm{GL}_{m_{j}}\left(E_{j}\right), I\right)$ compatible with supports, as in the latter algebra the characteristic function of any totally positive double coset is invertible. It thus suffices to establish this second claim.

Let $\bar{H}\left(\mathrm{GL}_{m_{j}}\left(E_{j}\right), I\right)$ be the subalgebra of $H\left(\mathrm{GL}_{m_{j}}\left(E_{j}\right), I\right)$ consisting of elements supported on double cosets of the form $I w^{\prime} I$ with $w^{\prime} \in W_{j}$, and $\bar{H}_{j}$ the subalgebra of $H_{j}$ supported on double cosets of the form $K^{\prime \prime} w K^{\prime \prime}$ with $w \in W_{j}$. Define $\bar{H}^{\circ}\left(\mathrm{GL}_{m_{j}}\left(E_{j}\right), I\right)$ and $\bar{H}_{j}^{\circ}$ analogously. The construction of [BK1, 5.6.1-5.6.4], yields a canonical isomorphism of $\bar{H}\left(\mathrm{GL}_{m_{j}}\left(E_{j}\right), I\right)$ with $\bar{H}_{j}$ compatible with supports, and their argument shows that this isomorphism sends $\bar{H}^{\circ}\left(\mathrm{GL}_{m_{j}}\left(E_{j}\right), I\right)$ to $\bar{H}_{j}^{\circ}$.

The extension of this isomorphism to an isomorphism of $H\left(\mathrm{GL}_{m_{j}}\left(E_{j}\right), I\right)$ with $H_{j}$ depends on a choice of element $x$ of $H_{j}$ supported on $K^{\prime \prime} \Pi_{j} K^{\prime \prime}$, where $\Pi_{j}$ is the element of the extended affine Weyl group denoted by $\Pi(\mathcal{B})$ in [BK1, Section 5.5] (here we consider $\Pi_{j}$ to be an element of $M_{j}$ ). Indeed, the main result of [BK1, Section 5.6] shows that given any such $x$ there is a unique isomorphism of $H\left(\mathrm{GL}_{m_{j}}\left(E_{j}\right), I\right)$ with $H_{j}$ sending the characteristic function of $I \Pi_{j} I$ to $x$ and extending the canonical isomorphism of $\bar{H}\left(\mathrm{GL}_{m_{j}}\left(E_{j}\right)\right)$ with $\overline{H_{j}}$.

Choose $x$ to lie in $H_{j}^{\circ}$, and to generate the $W(k)$-submodule of $H_{j}^{\circ}$ supported on $K^{\prime \prime} \Pi_{j} K^{\prime \prime}$ (note that the latter is free of rank one over $W(k)$; see the proof of [BK1, 5.6.7]). By the argument of [BK1, 5.6.8], such an $x$ is invertible in $H_{j}^{\circ}$.

As $H^{\circ}\left(\mathrm{GL}_{m_{j}}\left(E_{j}\right), I\right)$ is generated by $\bar{H}^{\circ}\left(\mathrm{GL}_{m_{j}}\left(E_{j}\right)\right)$, together with the characteristic function of $I \Pi_{j} I$ and its inverse, the isomorphism $H\left(\mathrm{GL}_{m_{j}}\left(E_{j}\right)\right.$, $I) \rightarrow H_{j}$ takes $H^{\circ}\left(\mathrm{GL}_{m_{j}}\left(E_{j}\right), I\right)$ to $H_{j}^{\circ}$. It thus remains to show that the image of $H^{\circ}\left(\mathrm{GL}_{m_{j}}\left(E_{j}\right), I\right)$ is all of $H_{j}^{\circ}$.

Note that for any element $w$ of $W_{j}$, and any integer $a$, left multiplication by the characteristic function of $I \Pi_{j} I$ is an isomorphism:

$$
H\left(\mathrm{GL}_{m_{j}}\left(E_{j}\right), I\right)_{I \Pi_{j}^{a} w I} \rightarrow H\left(\mathrm{GL}_{m_{j}}\left(E_{j}\right), I\right)_{I \Pi_{j}^{a+1} w I} .
$$

Thus left multiplication by $x$ maps $\left(H_{j}^{\circ}\right)_{K^{\prime \prime} \Pi_{j}^{a} w^{\prime} K^{\prime \prime}}$ to $\left(H_{j}^{\circ}\right)_{K^{\prime \prime} \Pi_{j}^{a+1} w K^{\prime \prime}}$, and this map is bijective since $x$ is invertible. On the other hand, every double coset on which 
$H_{j}^{\circ}$ is nonzero has the form $\Pi_{j}^{a} w$ for some $a$ and some $w$. Thus $H_{j}^{\circ}$ is generated by $x$ and $\bar{H}_{k}^{\circ}$ and the result follows.

Our next step is to translate this detailed structure theory for $H\left(G, K^{\prime \prime}, \tau^{\prime \prime}\right)$ into a corresponding theory for $H\left(G, K_{P}, \tau_{P}\right)$, via the isomorphisms of Theorem 8.2. (This is not necessary in the depth zero case, as in that case we have $K_{P}=K^{\prime \prime}$ and $\tau_{P}=\tau^{\prime \prime}$.) To do so we must study the intertwining of $\tau_{P}$ and of $\tau$.

LEMMA 8.5. Let $\tilde{P}$ be the preimage of $\bar{P}$ under the map

$$
\mathrm{GL}_{n / e f}\left(\mathcal{O}_{E}\right) \rightarrow \mathrm{GL}_{n / e f}\left(\mathbb{F}_{q^{f}}\right) \text {. }
$$

(1) Let $\xi$ be a $W(k)$-representation of $K$ trivial on $K_{1}$, and let $g$ be an element of $G$ that intertwines $\tilde{\kappa} \otimes \xi$. Then $g$ lies in $K \mathrm{GL}_{n / e f}(E) K$. Moreover, if $g$ lies in $\mathrm{GL}_{n / e f}(E)$, then $g$ intertwines $\xi$ when $\xi$ is considered as a representation of $\mathrm{GL}_{n / e f}\left(\mathcal{O}_{E}\right)$ inflated from $\mathrm{GL}_{n / e f}\left(\mathbb{F}_{q^{f}}\right)$, and there is a natural isomorphism:

$$
I_{g}(\tilde{\kappa} \otimes \xi) \cong I_{g}(\tilde{\kappa}) \otimes I_{g}(\xi)
$$

(2) Let $\xi$ be a $W(k)$-representation of $K_{P}$ trivial on $K_{1}$, and let $g$ be an element of $G$ that intertwines $\left.\tilde{\kappa}\right|_{K_{P}} \otimes \xi$. Then $g$ lies in $K_{P} \mathrm{GL}_{n / e f}(E) K_{P}$. Moreover, if $g$ lies in $\mathrm{GL}_{n / e f}(E)$, and then $g$ intertwines $\xi$ when $\xi$ is considered as a representation of $\tilde{P}$ inflated from $\bar{P}$, and there is a natural isomorphism:

$$
I_{g}\left(\left.\tilde{\kappa}\right|_{K_{P}} \otimes \xi\right) \cong I_{g}(\tilde{\kappa}) \otimes I_{g}(\xi) .
$$

Proof. Case (1) is almost precisely [BK1, Proposition 5.3.2], except that the coefficient space here is $W(k)$ rather than $\mathbb{C}$. In spite of this the argument of [BK1] adapts without difficulty, and the argument in case (2) is identical.

COROLlaRY 8.6. Let $g$ be an element of $G$ that intertwines $\tau_{P}$. Then $g$ lies in the double coset $K_{P} \mathrm{GL}_{n / e f}(E) K_{P}$. Moreover, if $g$ lies in $K_{P} W_{\bar{M}}(s) Z K_{P}$, then $I_{g}\left(\tau_{P}\right)$ is one-dimensional.

Proof. The previous lemma shows that $g$ lies in $K_{P} \mathrm{GL}_{n / e f}(E) K_{P}$, so the first statement is clear. For the second statement, it suffices to consider $g$ in $W_{\bar{M}}(s) Z$. For such $g, I_{g}\left(\tau_{P}\right)$ is equal to $I_{g}\left(\mathrm{St}_{\bar{M}, s}\right)$, where $\mathrm{St}_{\bar{M}, s}$ is considered as a representation of the parahoric subgroup $\tilde{P}$ of $\mathrm{GL}_{n / e f}(E)$ inflated from $\bar{M}$. We thus have

$$
I_{g}\left(\mathrm{St}_{\bar{M}, s}\right)=\operatorname{Hom}_{\tilde{P} \cap g \tilde{P} g^{-1}}\left(\mathrm{St}_{\bar{M}, s}, \mathrm{St}_{\bar{M}, s}^{g}\right) .
$$


It is easy to see that for $g$ in $W_{\bar{M}}(s) Z$, we have a surjection

$$
\tilde{P} \cap g \tilde{P} g^{-1} \rightarrow \bar{M}
$$

induced by the surjection of $\tilde{P}$ onto $\bar{M}$, and that the representations $\mathrm{St}_{\bar{M}, s}$ and $\mathrm{St}_{\bar{M}}^{g}, s$, when considered as representations of $\tilde{P} \cap g \tilde{P} g^{-1}$, are both inflated from $\mathrm{St}_{\bar{M}}$ via this surjection. The result follows.

As a result we obtain:

PROPOSITION 8.7. The isomorphism:

$$
H\left(G, K^{\prime \prime}, \tau^{\prime \prime}\right) \cong H\left(G, K_{P}, \tau_{P}\right)
$$

of Theorem 8.2 is support-preserving, in the sense that for all $g$ in $\mathrm{GL}_{n / e f}(E)$, it induces an isomorphism:

$$
H\left(G, K^{\prime \prime}, \tau^{\prime \prime}\right)_{K^{\prime \prime} g K^{\prime \prime}} \cong H\left(G, K_{P}, \tau_{P}\right)_{K_{P} g K_{P}} .
$$

Proof. For any $g$ in $G$, we have an isomorphism:

$$
\bigoplus_{g^{\prime}} H\left(G, K^{\prime \prime}, \tau^{\prime \prime}\right)_{K^{\prime \prime} g^{\prime} K^{\prime \prime}} \cong H\left(G,\left(\mathfrak{A}^{\prime}\right)^{\times}, \mathrm{c}-\operatorname{Ind}_{K^{\prime \prime}}^{\left(\mathfrak{A}^{\prime}\right)^{\times}} \tau^{\prime \prime}\right)_{\left(\mathfrak{A}^{\prime}\right)^{\times} g\left(\mathfrak{A}^{\prime}\right)^{\times}}
$$

where $g^{\prime}$ lies in a set of representatives for the double cosets $K^{\prime \prime} g^{\prime} K^{\prime \prime}$ in $\left(\mathfrak{A}^{\prime}\right)^{\times} g\left(\mathfrak{A}^{\prime}\right)^{\times}$. An easy calculation shows that if $g$ and $g^{\prime}$ lie in $W_{\bar{M}}(s) Z$, and

$$
\left(\mathfrak{A}^{\prime}\right)^{\times} g\left(\mathfrak{A}^{\prime}\right)^{\times}=\left(\mathfrak{A}^{\prime}\right)^{\times} g^{\prime}\left(\mathfrak{A}^{\prime}\right)^{\times},
$$

then $K^{\prime \prime} g K^{\prime \prime}=K^{\prime \prime} g^{\prime} K^{\prime \prime}$ and $K_{P} g K_{P}=K_{P} g^{\prime} K_{P}$. As $H\left(G, K^{\prime \prime}, \tau^{\prime \prime}\right)$ is supported on $K^{\prime \prime} W_{\bar{M}}(s) Z K^{\prime \prime}$, it follows that $H\left(G,\left(\mathfrak{A}^{\prime}\right)^{\times}, \mathrm{c}-\operatorname{Ind}_{K^{\prime \prime}}^{\left(\mathfrak{A}^{\prime}\right)^{\times}} \tau^{\prime \prime}\right)$ is supported on the double cosets $\left(\mathfrak{A}^{\prime}\right)^{\times} W_{\bar{M}}(s) Z\left(\mathfrak{A}^{\prime}\right)^{\times}$. Moreover, each $H\left(G, K^{\prime \prime}, \tau^{\prime \prime}\right)_{K^{\prime \prime} g K^{\prime \prime}}$ is onedimensional for $g$ in $W_{\bar{M}}(s) Z$. It follows that $H\left(G,\left(\mathfrak{A}^{\prime}\right)^{\times}, \mathrm{c}-\operatorname{Ind}_{K^{\prime \prime}}^{\left(\mathfrak{A}^{\prime}\right)^{\times}} \tau^{\prime \prime}\right)_{\left(\mathfrak{A}^{\prime}\right)^{\times} g\left(\mathfrak{A}^{\prime}\right)^{\times}}$ is as well.

On the other hand, for $g$ in $G$ we also have an isomorphism

$$
\bigoplus_{g^{\prime}} H\left(G, K_{P}, \tau_{P}\right)_{K_{P} g^{\prime} K_{P}} \cong H\left(G,\left(\mathfrak{A}^{\prime}\right)^{\times}, \mathrm{c}-\operatorname{Ind}_{K_{P}}^{\left(\mathfrak{A}^{\prime}\right)^{\times}} \tau_{P}\right)_{\left(\mathfrak{A}^{\prime}\right)^{\times} g\left(\mathfrak{A}^{\prime}\right)^{\times}},
$$

where $g^{\prime}$ runs over a set of representatives for the cosets $K_{P} g^{\prime} K_{P}$ in $\left(\mathfrak{A}^{\prime}\right)^{\times} g\left(\mathfrak{A}^{\prime}\right)^{\times}$. For $g$ in $W_{\bar{M}}(s) Z$, the right-hand side is one-dimensional, and the summand on the left corresponding to $g^{\prime}=g$ is also one-dimensional. On the other hand, if $g$ doesn't lie in $\left(\mathfrak{A}^{\prime}\right)^{\times} W_{\bar{M}}(s) Z\left(\mathfrak{A}^{\prime}\right)^{\times}$then the right-hand side is zero, so all of the summands on the left vanish as well. It follows that $H\left(G, K_{P}, \tau_{P}\right)$ is supported on $K_{P} W_{\bar{M}}(s) Z K_{P}$, and that for $g$ in $W_{\bar{M}}(s) Z$, the isomorphisms above identify $H\left(G, K_{P}, \tau_{P}\right)_{K_{P} g K_{P}}$ with $H\left(G, K^{\prime \prime}, \tau^{\prime \prime}\right)_{K^{\prime \prime} g K^{\prime \prime}}$ as required. 
With Proposition 8.7 established, the remainder of the argument goes more or less exactly as in Section 6. The next step is to understand the relationship between $H\left(G, K_{P}, \tau_{P}\right)$ and $H\left(G, K, \tilde{\kappa} \otimes \mathrm{St}_{s}\right)$. As $\mathrm{St}_{s}$ is a direct summand of $I_{s}$, $\mathrm{c}-\operatorname{Ind}_{K}^{G} \tilde{\kappa} \otimes \mathrm{St}_{s}$ is a direct summand of $\mathrm{c}-\operatorname{Ind}_{K}^{G} \tilde{\kappa} \otimes I_{s}$. We therefore obtain a map:

$$
Z\left(H\left(G, K_{P}, \tau_{P}\right)\right) \rightarrow H\left(G, K, \tilde{\kappa} \otimes \mathrm{St}_{s}\right)
$$

that is support-preserving in the sense that elements supported on a double coset $K_{P} g K_{P}$ for some $g \in \mathrm{GL}_{n / e f}(E)$ get sent to elements of $H\left(G, K, \tilde{\kappa} \otimes \mathrm{St}_{s}\right)$ supported on $K g K$. As in depth zero, we have:

Proposition 8.8. The map $Z\left(H\left(G, K, \tilde{\kappa} \otimes I_{s}\right)\right) \rightarrow H\left(G, K, \tilde{\kappa} \otimes \mathrm{St}_{s}\right)$ is injective.

Proof. The proof of this is identical to the proof of Proposition 6.8.

To show that this map is an isomorphism, it remains to compare the dimensions of $H\left(G, K, \tilde{\kappa} \otimes \mathrm{St}_{s}\right)_{K g K}$ and $Z\left(H\left(G, K, \tilde{\kappa} \otimes I_{s}\right)\right)_{K g K}$ for a suitable set of $g$. This is essentially an intertwining calculation, analogous to the one done in Section 6, except that we must now respect the distinction between $\mathrm{GL}_{n / e f}(E)$ and $\mathrm{GL}_{n}(F)$, which introduces a few subtleties:

Fix an element $g$ of $M \cap \mathrm{GL}_{n / e f}(E)$. For such a $g$, let $\bar{P}_{g}$ be the image of $g \mathrm{GL}_{n / e f}\left(\mathcal{O}_{E}\right) g^{-1} \cap \mathrm{GL}_{n / e f}\left(\mathcal{O}_{E}\right)$ in $\bar{G}$. Then $\bar{P}_{g}$ is a parabolic subgroup with unipotent radical $\bar{U}_{g}$, and Levi $\bar{M}_{g}$.

Let $\tilde{P}_{g}$ be the subgroup $g \mathrm{GL}_{n / e f}\left(\mathcal{O}_{E}\right) g^{-1} \cap \mathrm{GL}_{n / e f}\left(\mathcal{O}_{E}\right)$ of $\mathrm{GL}_{n / e f}\left(\mathcal{O}_{E}\right)$. Then $g^{-1} \tilde{P}_{g} g$ is also a subgroup of $\mathrm{GL}_{n / e f}\left(\mathcal{O}_{E}\right)$, and its image under the reduction map to $\bar{G}$ is opposite parabolic $\bar{P}_{g}^{\circ}$ of $\bar{P}_{g}$. Let $\tilde{U}_{g}$ be the preimage of $\bar{U}_{g}$ in $\tilde{P}_{g}$; then for any $u$ in $\tilde{U}_{g}$, the conjugate $g^{-1} u g$ maps to the identity under the composition $g^{-1} \tilde{P}_{g} g \rightarrow \bar{P}_{g} \rightarrow \bar{M}_{g}$. The conjugation map $\tilde{P}_{g} \rightarrow g^{-1} \tilde{P}_{g} g$ thus descends to a map $\bar{M}_{g} \rightarrow \bar{M}_{g}$; we say $g$ is $\bar{M}_{g}$-central if the resulting automorphism of $\bar{M}_{g}$ is trivial. It is clear that for any $g$, there exists a $k$ in $\mathrm{GL}_{n / e f}\left(\mathcal{O}_{E}\right)$ such that $g k$ is $\bar{M}_{g}$-central. We then have:

LEMMA 8.9. Let $g$ be an element of $M \cap \mathrm{GL}_{n / e f}(E)$, and let $\xi$ be a $W(k)[\bar{G}]$ module considered as a module over $W(k)\left[\mathrm{GL}_{n / \text { ef }}\left(\mathcal{O}_{E}\right)\right]$ by inflation. Then $I_{g}(\xi)$ is free of rank one over $\operatorname{End}_{W(k)\left[\bar{M}_{g}\right]}\left(r_{\bar{G}}^{\bar{P}_{g}} \xi\right)$. Moreover, if $g$ is $\bar{M}_{g}$-central, we have a natural isomorphism:

$$
I_{g}(\xi) \cong \operatorname{End}_{W(k)\left[\bar{M}_{g}\right]}\left(r_{\bar{G}}^{\bar{P}_{g}} \xi\right) .
$$


Proof. By definition, $I_{g}(\xi)=\operatorname{Hom}_{W(k)\left[\tilde{P}_{g}\right]}\left(\xi, \xi^{g}\right)$. Note that for $u$ in $\tilde{U}_{g}$, the element $g^{-1} u g$ acts trivially on $\xi$. Thus $u$ acts trivially on $\xi^{g}$. In particular, any element of $I_{g}(\xi)$ gives rise to a map:

$$
\operatorname{Hom}_{W(k)\left[\tilde{P}_{g}\right]}\left(r_{\bar{G}}^{\bar{P}_{g}} \xi, r_{\bar{G}}^{\bar{P}_{g}} \xi^{g}\right) .
$$

Conversely, any such map gives rise to an element of $I_{g}(\xi)$. (Here we are identifying the $\bar{U}_{g}$-invariants of $\xi$ with the $\bar{U}_{g}$-coinvariants via the natural map from invariants to coinvariants. Moreover, the representation $r_{\bar{G}} \bar{P}_{g}$ is a representation of $\bar{M}_{g}$ considered as a representation of $\tilde{P}_{g}$ by inflation.) As conjugation by $g$ descends to an inner automorphism of $\bar{M}_{g}, r_{\bar{G}} \bar{P}_{g} \xi^{g}$ is isomorphic to $r_{\bar{G}}^{\bar{P}_{g}} \xi$, and we can take this isomorphism to be the identity when $g$ is $\bar{M}_{g}$-central. The result is then clear.

Let $z$ be an element of $Z$. Then our fixed maximal torus of $\bar{G}$ is a maximal torus of $\bar{M}_{g}$. As in Section 6, we define $W\left(\bar{M}, \bar{M}_{z}\right)$ as the set of $w$ in $W(\bar{G})$ such that $w \bar{M} w^{-1}$ is contained in $\bar{M}_{z}$. We then have:

Proposition 8.10. The spaces $H\left(G, K, \tilde{\kappa} \otimes \mathrm{St}_{s}\right)$ and $H\left(G, K, \tilde{\kappa} \otimes i \frac{\bar{G}}{P} \mathrm{St}_{\bar{M}, s}\right)$ are supported on $K Z K$. Moreover, for $z$ in $Z$, we have:

$$
\begin{gathered}
H\left(G, K, \tilde{\kappa} \otimes I_{s}\right)_{K z K} \cong I_{z}(\tilde{\kappa}) \otimes \operatorname{End}_{\overline{\mathcal{K}}_{\left[\bar{M}_{z}\right]}}\left(\oplus_{w} I_{\bar{M}_{z}, w s w^{-1}}\right) \\
H\left(G, K, \tilde{\kappa} \otimes \operatorname{St}_{s}\right)_{K z K} \cong I_{z}(\tilde{\kappa}) \otimes \operatorname{End}_{\overline{\mathcal{K}}\left[\bar{M}_{z}\right]}\left(\oplus_{w^{\prime}} \operatorname{St}_{\bar{M}_{z}, w^{\prime} s\left(w^{\prime}\right)^{-1}}\right)
\end{gathered}
$$

where $w$ runs over a set of representatives for $W\left(\bar{M}_{z}\right) \backslash W\left(\bar{M}, \bar{M}_{z}\right)$, and $w^{\prime}$ runs over a set of representatives for $W\left(\bar{M}_{z}\right) \backslash W\left(\bar{M}, \bar{M}_{z}\right) / W_{\bar{M}}(s)$.

Proof. By Lemma 8.5, it suffices to compute the spaces $I_{z}\left(I_{s}\right)$ and $I_{z}\left(\mathrm{St}_{s}\right)$. Note that for any $z$ in $Z$, it is clear that $z$ is $\bar{M}_{z}$-central. The result is thus immediate from Lemma 8.9, together with Propositions 5.3 and 5.5.

As a result, exactly as in Section 6, we have:

Corollary 8.11. For $z \in Z$, the dimension of $H\left(G, K, \tilde{\kappa} \otimes S t_{s}\right)_{K z K}$ is equal to the cardinality of $W\left(\bar{M}_{z}\right) \backslash W\left(\bar{M}, \bar{M}_{z}\right) / W_{\bar{M}}(s)$.

Proof. The modules $\mathrm{St}_{\bar{M}_{z}, w s w^{-1}}$ and $\mathrm{St}_{\bar{M}_{z},\left(w^{\prime}\right) s\left(w^{\prime}\right)^{-1}}$ are isomorphic if, and only if, $w$ and $w^{\prime}$ represent the same class in $W\left(\bar{M}_{z}\right) \backslash W\left(\bar{M}, \bar{M}_{z}\right) / W_{\bar{M}}(s)$. It follows that

$$
\operatorname{dim}_{\overline{\mathcal{K}}} \operatorname{End}_{\overline{\mathcal{K}}_{\left[\bar{M}_{z}\right]}}\left(\oplus_{w} \operatorname{St}_{\bar{M}_{z}, w s w^{-1}}\right)=\# W\left(\bar{M}_{z}\right) \backslash W\left(\bar{M}, \bar{M}_{z}\right) / W_{\bar{M}}(s)
$$

and the result follows by Proposition 8.10. 
It remains to compute the dimension of the subspace of $H\left(G, K, \tilde{\kappa} \otimes I_{s}\right)$ that is central and supported on $K g K$. The argument here is more or less identical to that of Section 6, via the identification of $H\left(G, K, \tilde{\kappa} \otimes I_{s}\right)$ with the tensor product of the $H_{j}$. This identification, together with Lemma 6.10, allows us to immediately deduce:

Proposition 8.12. For any $z$ in $Z$, the space of central elements of $H\left(G, K_{P}\right.$, $\left.\tau_{P}\right)$ supported on the union of double cosets of the form $K_{P} w z w^{\prime} K_{P}$ with $w, w^{\prime}$ in $W_{\bar{M}}(s)$ is one-dimensional. Moreover, the sum of these spaces as $z$ varies is the entire center of $H\left(G, K_{P}, \tau_{P}\right)$.

We can thus conclude:

THEOREM 8.13. The map:

$$
Z\left(H\left(G, K_{P}, \tau_{P}\right)\right) \rightarrow H\left(G, K, \tilde{\kappa} \otimes \mathrm{St}_{s}\right)
$$

is an isomorphism.

Proof. By the injectivity of this map, and Corollary 8.11, it suffices to show that for each $z$ in $Z$, the subspace of $Z\left(H\left(G, K_{P}, \tau_{P}\right)\right)$ supported on $K z K$ has dimension equal to the cardinality of $W\left(\bar{M}_{z} \backslash W\left(\bar{M}, \bar{M}_{z}\right) / W_{\bar{M}}(s)\right.$. This is immediate from Propositions 8.12 and 6.11.

As in 6, we may deduce from this that the map $A_{M, \pi} \rightarrow H\left(G, K, \tilde{\kappa} \otimes \mathrm{St}_{s}\right)$ giving the action of $A_{M, \pi}$ on c-Ind ${ }_{K}^{G} \tilde{\kappa} \otimes \mathrm{St}_{s}$ is an isomorphism.

\section{Structure of $\operatorname{End}_{G}\left(\mathcal{P}_{K, \tau}\right)$}

Our next step will be to understand the endomorphism ring $\operatorname{End}_{W(k)[G]}\left(\mathcal{P}_{K, \tau}\right)=$ $H\left(G, K, \mathcal{P}_{\kappa \otimes \sigma}\right)$ for an arbitrary $\kappa$. Mostly, we will follow the arguments of Section 7 in the depth zero case, with some necessary modifications. However, it will be useful to make some additional observations, which we omitted from Section 7 because their proof does not appreciably simplify in the depth zero setting.

To simplify notation, let $E_{\sigma}$ be the $\operatorname{ring} \operatorname{End}_{W(k)[K]}\left(\mathcal{P}_{\sigma}\right)$, and let $E_{K, \tau}$ be the ring $\operatorname{End}_{W(k)[G]}\left(\mathcal{P}_{K, \tau}\right)$. By Proposition 5.8, $E_{\sigma}$ is a reduced commutative $W(k)$-algebra that is free of finite rank over $W(k)$.

We have a direct sum decomposition:

$$
\mathcal{P}_{\sigma} \otimes \overline{\mathcal{K}} \cong \bigoplus_{s: s^{\mathrm{reg}^{\prime}}=s^{\prime}} \mathrm{St}_{s}
$$


where $s^{\prime}$ is the $\ell$-regular semisimple element of $\bar{G}$ corresponding to the representation $\sigma$. This yields a decomposition:

$$
\mathcal{P}_{K, \tau} \otimes \overline{\mathcal{K}}=\bigoplus_{s: \mathrm{s}^{\mathrm{reg}}=s^{\prime}} \mathrm{c}-\operatorname{Ind}_{K}^{G} \tilde{\kappa} \otimes \mathrm{St}_{s} .
$$

Proposition 9.1. Let $g$ be an element of $G$ that intertwines $\mathcal{P}_{K, \tau}$. Then $g$ lies in $K \mathrm{GL}_{n / e f}(E) K$, and we have a direct sum decomposition:

$$
I_{g}\left(\mathcal{P}_{K, \tau}\right) \otimes \overline{\mathcal{K}}=\bigoplus_{s: s^{\mathrm{reg}}=s^{\prime}} I_{g}\left(\tilde{\kappa} \otimes \mathrm{St}_{s}\right)
$$

Moreover, if $g$ lies in $\mathrm{GL}_{n / e f}$ and is $\bar{M}_{g}$-central, then there is a natural isomorphism:

$$
I_{g}\left(\mathcal{P}_{K, \tau}\right) \cong I_{g}(\tilde{\kappa}) \otimes \operatorname{End}_{W(k)\left[\bar{M}_{g}\right]}\left(r_{\bar{G}}^{\bar{P}_{g}} \mathcal{P}_{\sigma}\right)
$$

Proof. Every statement other than the decomposition is a direct consequence of Lemma 8.9. As for the direct sum decomposition, the decomposition:

$$
P_{\sigma} \otimes \overline{\mathcal{K}}=\bigoplus_{s: s^{\mathrm{reg}}=s^{\prime}} \mathrm{St}_{s}
$$

yields a decomposition

$$
r_{\bar{G}}^{\bar{P}} \mathcal{P}_{\sigma} \otimes \overline{\mathcal{K}}=\bigoplus_{s: s^{\mathrm{reg}}=s^{\prime}} r_{\bar{G}}^{\bar{P}} \mathrm{St}_{s}
$$

From Proposition 5.3 we see that no two irreducible summands of the right-hand side are isomorphic to each other, so any endomorphism of the right-hand side preserves each of the summands. We thus have a decomposition:

$$
\operatorname{End}_{W(k)\left[\bar{M}_{g}\right]}\left(r_{\bar{G}}^{\bar{P}_{g}} \mathcal{P}_{\sigma}\right)=\bigoplus_{s: s^{\text {reg }}=s^{\prime}} \operatorname{End}_{W(k)\left[\bar{M}_{g}\right]}\left(r_{\bar{G}}^{\bar{P}_{g}} \mathrm{St}_{s}\right) .
$$

Tensoring both sides with $I_{g}(\tilde{\kappa})$ yields the desired result.

COROLlaRY 9.2. The action of $E_{K, \tau} \otimes \overline{\mathcal{K}}$ on the direct sum decomposition

$$
\mathcal{P}_{K, \tau} \otimes \overline{\mathcal{K}}=\bigoplus_{s: s^{\mathrm{reg}}=s^{\prime}} \mathrm{c}-\operatorname{Ind}_{K}^{G} \tilde{\kappa} \otimes \mathrm{St}_{s}
$$

preserves each summand, and thus yields an isomorphism:

$$
E_{K, \tau} \otimes \overline{\mathcal{K}} \cong \prod_{s: s^{\text {reg }}=s^{\prime}} H\left(G, K, \tilde{\kappa} \otimes \mathrm{St}_{s}\right) .
$$


In particular, $E_{K, \tau}$ is reduced, commutative, and $\ell$-torsion free. Moreover, Spec $E_{K, \tau}$ is connected.

Proof. The direct sum decomposition follows immediately from Proposition 9.1 by summing over the elements supported on $K g K$ for each $g$. The embedding follows from the absence of $\ell$-torsion in $E_{K, \tau}$ (which is immediate from the fact that $\mathcal{P}_{K, \tau}$ is projective). Reducedness and commutativity of $E_{K, \tau}$ then follow from the corresponding results for $H\left(G, K, \tilde{\kappa} \otimes \mathrm{St}_{s}\right)$.

For the last claim, suppose that we had a nontrivial idempotent in $E_{K, \tau}$. Then, since the spectrum of $H\left(G, K, \tilde{\kappa} \otimes \mathrm{St}_{s}\right)$ is connected for all $s$, such an idempotent maps to either zero or one in each $H\left(G, K, \tilde{\kappa} \otimes \mathrm{St}_{s}\right)$, and hence lies in $E_{\sigma} \otimes \mathcal{K}$. But $E_{\sigma}$ is saturated in $E_{K, \tau}$; that is, if $\ell x$ lies in $E_{\sigma}$, then so does $x$. Thus we would obtain a nontrivial idempotent in $E_{\sigma}$, which is impossible since $E_{\sigma}$ is local.

We now turn to questions that have already been addressed in the depth zero case in Section 7. As in that setting, it will be necessary to understand families of cuspidal representations of $\mathrm{GL}_{i}(F)$ for various $i$, all of which have supercuspidal support inertially equivalent to some power of a fixed supercuspidal representation over $k$. Fix an integer $n_{1}$, and let $\left(K_{1}, \tau_{1}\right)$ be a maximal distinguished supercuspidal $k$-type for $\mathrm{GL}_{n_{1}}(F)$. We have $\tau_{1}=\kappa_{1} \otimes \sigma_{1}$, where $\sigma_{1}$ is the supercuspidal representation of $\mathrm{GL}_{n_{1} / e f}\left(\mathbb{F}_{q^{f}}\right)$ attached to an $\ell-$ regular element $s_{1}^{\prime}$ of $\mathrm{GL}_{n_{1} / e f}\left(\mathbb{F}_{q^{f}}\right)$ with irreducible characteristic polynomial.

Let $f^{\prime}$ be the product $f \operatorname{deg}\left(s_{1}^{\prime}\right)$, and recall that $e_{q f^{\prime}}$ is the order of $q^{f^{\prime}}$ modulo $\ell$. Let $m$ be an element of the set $\left\{1, e_{q^{f^{\prime}}}, \ell e_{q^{f^{\prime}}}, \ell^{2} e_{q^{f^{\prime}}}, \ldots\right\}$. For precisely such $m$, the generic representation $\sigma_{m}$ of $\mathrm{GL}_{n_{1} m / e f}\left(\mathbb{F}_{q^{f}}\right)$ corresponding to a block matrix consisting of $m$ copies of $s_{1}^{\prime}$ is cuspidal.

In Section 7 the choice of such a $\sigma_{m}$ sufficed to define a depth zero type in $\mathrm{GL}_{n_{1} m}(F)$. Here we must work harder: for any suitable $m$ we must also define a representation $\kappa_{m}$ of a suitable compact open subgroup of $\operatorname{GL}_{n_{1} m}(F)$. We do so as follows: the data giving rise to the type $\left(K_{1}, \tau_{1}\right)$ consist of an extension $E$ of $F$ of ramification index $e$ and residue class degree $f$, a stratum $\left[\mathfrak{A}_{1}, n\right.$, $0, \beta]$, with $E=F[\beta]$, and a character $\theta$ in $\mathcal{C}\left(\mathfrak{A}_{1}, 0, \beta\right)$. Then $\theta$ gives rise to an endo-equivalence class $(\Theta, 0, \beta)$. Let $\mathfrak{A}_{m}$ be the maximal order $M_{m}\left(\mathfrak{A}_{1}\right)$ of $M_{n_{1} m}(F)$; then the endo-class $(\Theta, 0, \beta)$ gives rise to a compact open subgroup $K_{m}$ of $\mathrm{GL}_{n_{1} m}(F)$ and a representation $\kappa_{m}$ of $K_{m}$. (This is slightly misleading: the representation $\kappa_{m}$ is in fact only well defined up to certain twists. For the moment we will let $\kappa_{m}$ be any representation of $K_{m}$ arising from the choice of $\mathfrak{A}_{m}$ and the endo-class $(\Theta, 0, \beta)$. Later we will make additional assumptions on $\kappa_{m}$ that pin it down precisely.

Let $\tau_{m}=\kappa_{m} \otimes \sigma_{m}$; then $\left(K_{m}, \tau_{m}\right)$ is a maximal distinguished cuspidal $k$-type in $\mathrm{GL}_{n_{1} m}(F)$. 
For each positive integer $m$, let $G_{m}$ be the group $\operatorname{GL}_{n_{1} m}(F)$ and let $\bar{G}_{m}$ be the group $\mathrm{GL}_{n_{1} m / e f}\left(\mathbb{F}_{q^{f}}\right)$. For each semisimple conjugacy class $s$ in $\bar{G}_{m}$ with $\ell$-regular part $\left(s_{1}^{\prime}\right)^{m}$, let $\bar{M}_{s}$ be the minimal split Levi of $\bar{G}_{m}$ containing $s$, let $M_{s}$ be the corresponding Levi of $G_{m}$, and let $Z_{s}$ be the subgroup of the center of $M_{s} \cap$ $\mathrm{GL}_{n_{1} m / e f}(E)$ consisting of diagonal matrices whose entries are powers of $\varpi_{E}$.

As in Section 7, we may define elements $\theta_{i, s}$ of $W(k)\left[Z_{s}\right]^{W_{\bar{M}_{s}}(s)}$ as follows:

DEFINITION 9.3. Let $\theta_{i, s}$ be the element of $Z_{s}$ given by the sum of the elements of $Z_{s}$ whose characteristic polynomial (as an element of $\operatorname{GL}_{n_{1} m / e f}(E)$ ) has the form $\left(t-\varpi_{E}\right)^{n_{1} i / e f}(t-1)^{n_{1}(m-i) / e f}$.

As in Section 7, it is possible that $\theta_{i, s}=0$.

Of particular interest to us will be the subgroups corresponding to $s=\left(s_{1}^{\prime}\right)^{m}$; we denote these by $\bar{M}_{0, m}, M_{0, m}$, and $Z_{0, m}$. For $i$ between 1 and $m$, let $z_{i, m}$ be an element of $Z_{0, m}$ with characteristic polynomial $\left(t-\varpi_{E}\right)^{n_{1} i / e f}(t-1)^{n_{1}(m-i) / e f}$.

For $m \in\left\{1, e_{q^{f^{\prime}}}, \ell e_{q^{f^{\prime}}}, \ldots\right\}$, and any conjugacy class $s$ in $\bar{G}_{m}$ with $\ell$-regular part $\left(s_{1}^{\prime}\right)^{m}$, set $E_{m}=E_{K_{m}, \tau_{m}}$. We have a chain of maps:

$$
E_{m} \rightarrow H\left(G_{m}, K_{m}, \tilde{\kappa}_{m} \otimes \mathrm{St}_{s}\right) \cong A_{M_{s}, \pi_{s}} \cong \overline{\mathcal{K}}\left[Z_{s}\right]^{W_{\bar{M}_{s}}(s)},
$$

where the first map arises from Corollary 9.2, the pair $\left(M_{s}, \pi_{s}\right)$ is in the inertial equivalence class determined by $\tilde{\kappa}_{m}$ and $s$, and the last isomorphism depends on a particular choice of $\left(M_{s}, \pi_{s}\right)$. As in Section 7 we will construct elements of $E_{m}$ whose action we can describe explicitly in terms of the $\theta_{i, s}$; this will require choosing a 'compatible family of cuspidals' analogous to the family constructed in Section 7 , in order to suitably normalize the maps to $\overline{\mathcal{K}}\left[Z_{s}\right]$ for all $s$.

Fix, once and for all, an absolutely irreducible integral supercuspidal representation $\pi_{s_{1}^{\prime}}$ of $\mathrm{GL}_{n_{1}}(F)$ containing $\left(K_{1}, \tilde{\kappa}_{1} \otimes \mathrm{St}_{s_{1}^{\prime}}\right)$. We will construct, for each $m \in\left\{1, e_{q f^{\prime}}, \ell e_{q^{f^{\prime}}}, \ldots\right\}$, and each irreducible conjugacy class $s$ in $\bar{G}_{m}$ such that $s^{\text {reg }}=\left(s_{1}^{\prime}\right)^{m}$, an absolutely irreducible cuspidal representation $\pi_{s}$ of $\mathrm{GL}_{n_{1} m}(F)$ containing $\left(K_{m}, \tilde{\kappa}_{m} \otimes \mathrm{St}_{s}\right)$. Our construction will mostly parallel that of Section 7, but as usual the nontriviality of $\tilde{\kappa}_{m}$ introduces complications.

We proceed as follows: note that $\left(K_{m}, \tilde{\kappa}_{m} \otimes \mathrm{St}_{s}\right)$ is a maximal distinguished cuspidal $\overline{\mathcal{K}}$-type. In particular, $H\left(G_{m}, K_{m}, \tilde{\kappa}_{m} \otimes \mathrm{St}_{s}\right)$ is isomorphic to $\overline{\mathcal{K}}\left[\Theta^{ \pm 1}\right]$, where $\Theta$ is an element of $H\left(G_{m}, K_{m}, \tilde{\kappa}_{m} \otimes \mathrm{St}_{s}\right)$ supported on $K_{m} z_{m, m} K_{m}$. We have an isomorphism:

$$
H\left(G_{m}, K_{m}, \tilde{\kappa}_{m} \otimes \mathrm{St}_{s}\right)_{K_{m} z_{m, m} K_{m}} \cong I_{z_{m, m}}\left(\tilde{\kappa}_{m}\right) \otimes \operatorname{End}_{\overline{\mathcal{K}}\left[\bar{G}_{m}\right]}\left(\mathrm{St}_{s}\right) .
$$

We also have an isomorphism:

$$
H\left(G_{m}, K_{m}, \tilde{\kappa}_{m} \otimes \operatorname{St}_{\left(s_{1}^{\prime}\right)^{m}}\right)_{K_{m} z_{m, m} K_{m}} \cong I_{z m, m}\left(\tilde{\kappa}_{m}\right) \otimes \operatorname{End}_{\overline{\mathcal{K}}_{\left[\bar{G}_{m}\right]}}\left(\mathrm{St}_{\left(s_{1}^{\prime}\right)^{m}}\right) .
$$


Let $\pi_{\left(s_{1}^{\prime}\right)^{m}}$ be the cuspidal representation $\pi_{s_{1}^{\prime}}^{\otimes m}$ of $M_{\left(s_{1}^{\prime}\right)^{m}}$. Such a choice gives an isomorphism of $H\left(G_{m}, K_{m}, \tilde{\kappa}_{m} \otimes \operatorname{St}_{\left(s_{1}^{\prime}\right)^{m}}\right)$ with $\overline{\mathcal{K}}\left[Z_{0, m}\right]^{W_{\bar{M}_{0, m}}\left(\left(s_{1}^{\prime}\right)^{m}\right)}$; the element $z_{m, m}$ of $\overline{\mathcal{K}}\left[Z_{0, m}\right]$ maps to an element of $H\left(G_{m}, K_{m}, \tilde{\kappa}_{m} \otimes \mathrm{St}_{\left(s_{1}^{\prime}\right)^{m}}\right)_{K_{m} z_{m, m} K_{m}}$. This element has the form $\phi \otimes 1$ for some $\phi \in I_{z m, m}\left(\tilde{\kappa}_{m}\right)$, where we regard 1 as the identity endomorphism of $\mathrm{St}_{\left(s_{1}^{\prime}\right)^{m}}$.

We may then consider the element $\phi \otimes 1$ of $I_{z_{m, m}}(\tilde{\kappa}) \otimes \operatorname{End}_{\left.\overline{\mathcal{K}}_{[} \bar{G}_{m}\right]}\left(\mathrm{St}_{s}\right)$. This gives us an element $\Theta$ of $H\left(G_{m}, K_{m}, \tilde{\kappa} \otimes \mathrm{St}_{s}\right)_{K_{m} z_{m, m} K_{m}}$. There is then a unique irreducible cuspidal representation $\pi_{s}$ of $G_{m}$ over $\overline{\mathcal{K}}$ that contains $\left(K_{m}, \tilde{\kappa} \otimes \mathrm{St}_{s}\right)$, on which $\Theta$ acts via the identity. Note that $\pi_{s}$ is integral.

We will refer to the family of cuspidal representations $\left\{\pi_{s}\right\}$ as the compatible family of cuspidals attached to our choice of $\pi_{s_{1}^{\prime}}$. The dependence on the choice of $\pi_{\left(s_{1}^{\prime}\right)}$ is mild; indeed, replacing $\pi_{\left(s_{1}^{\prime}\right)}$ by an unramified twist $\pi_{\left(s_{1}^{\prime}\right)} \otimes(\chi \circ$ det $)$ simply twists each $\pi_{s}$ by $\chi \circ$ det as well. Note that (for the moment) there is also a dependence on the $\kappa_{m}$ we have chosen; this will be resolved when we pin down the $\kappa_{m}$ precisely.

For each $m$, and each reducible conjugacy class $s$ in $\bar{G}_{m}$, we define an irreducible cuspidal representation $\pi_{s}$ of $M_{s}$, by taking $\pi_{s}$ to be the tensor product of the cuspidal representations $\pi_{s_{i}}$ defined above, where the $s_{i}$ are the irreducible factors of $s$.

We can now state a key result of this section, analogous to Theorem 7.2:

THEOREM 9.4. For each $m$, there exists a subalgebra $C_{K_{m}, \tau_{m}}$ of $E_{K_{m}}, \tau_{m}$, generated over $W(k)$ by elements $\Theta_{1, m}, \ldots, \Theta_{m, m}$ and $\left(\Theta_{m, m}\right)^{-1}$, such that for any $s$ with $s^{\mathrm{reg}}=\left(s_{1}^{\prime}\right)^{m}$, the composed map:

$$
C_{K_{m}, \tau_{m}} \rightarrow A_{M_{s}, \pi_{s}} \cong \overline{\mathcal{K}}\left[Z_{s}\right]^{W_{\bar{M}_{s}}(s)}
$$

takes $\Theta_{i, m}$ to $\theta_{i, s}$. (Here the left-hand map is the map giving the action of $E_{K_{m}, \tau_{m}}$ on the summand $\left(\mathcal{P}_{K_{m}, \tau_{m}} \otimes \overline{\mathcal{K}}\right)_{M_{s}, \pi_{s}}$ of $\mathcal{P}_{K_{m}, \tau_{m}} \otimes \overline{\mathcal{K}}$.)

Note that this property characterizes the $\Theta_{i, m}$ uniquely. The basic strategy, as in the depth zero case, is to construct the $\Theta_{i, m}$ inductively via $G$-covers. The case $m=1$ is easy: we have an isomorphism

$$
H\left(G_{1}, K_{1}, \tilde{\kappa}_{1} \otimes \mathcal{P}_{\sigma_{1}}\right)_{K_{1} z_{1,1} K_{1}} \cong I_{z_{1,1}}\left(\tilde{\kappa}_{1}\right) \otimes \operatorname{End}_{W(k)\left[\bar{G}_{1}\right]}\left(\mathcal{P}_{\sigma_{1}}\right) .
$$

We define $\Theta_{1,1}$ to be the unique element of the form $\phi \otimes 1$, for some $\phi$ in $I_{z_{1,1}}\left(\tilde{\kappa}_{1}\right)$, that acts on the quotient $\pi_{\left(s_{1}^{\prime}\right)}$ of $\mathcal{P}_{K_{1}, \tau_{1}} \otimes \overline{\mathcal{K}}$ via the identity. It is clear from our construction of the $\pi_{s}$ that this $\Theta_{1,1}$ has the desired property.

We now turn to the inductive part of the argument. This will proceed via a $G$-cover argument, along the lines of the construction in Section 7. However, 
the argument is considerably more delicate because of the presence of the $\tilde{\kappa}_{m}$. In particular, the same complications that arose in Section 8 (as compared to Section 6) will arise here.

Let $m^{\prime}$ be the largest element of $\left\{1, e_{q^{f^{\prime}}}, \ell e_{q^{f^{\prime}}}, \ldots\right\}$ strictly less than $m$, and set $j=m / m^{\prime}$. Let $V_{m^{\prime}}$ be the $F$-vector space on which $G_{m^{\prime}}$ acts, and identify $V_{m}$ with the direct sum of $j$ copies $V_{m^{\prime}, 1}, \ldots, V_{m^{\prime}, j}$ of $V_{m^{\prime}}$. Let $M$ be the Levi subgroup of $G_{m}$ preserving this direct sum decomposition, and let $P$ be the parabolic preserving the flag

$$
V_{m^{\prime}, 1} \subset V_{m^{\prime}, 1}+V_{m^{\prime}, 2} \subset \cdots \subset V_{m^{\prime}, 1}+\cdots+V_{m^{\prime}, j} .
$$

The groups $P=M U$ give rise to subgroups $\bar{P}=\overline{M U}$ of $\bar{G}_{m}$ in the usual way.

We have maximal orders $\mathfrak{A}_{m}$ of $G_{m}$ and $\mathfrak{A}_{m^{\prime}}$ of $G_{m^{\prime}}$ attached to the types $\left(K_{m}, \tau_{m}\right)$ and $\left(K_{m^{\prime}}, \tau_{m^{\prime}}\right)$. The procedure of [BK3, 7.2] also yields an order $\mathfrak{A}_{m}^{\prime}$ contained in $\mathfrak{A}_{m}$ attached to the flag defined above. Set $K_{m}^{\prime}=J\left(\beta, \mathfrak{A}_{m}^{\prime}\right)$, let $K_{m}^{\prime \prime}$ be the subgroup $\left(J\left(\beta, \mathfrak{A}_{m}^{\prime}\right) \cap P\right) H^{1}\left(\beta, \mathfrak{A}_{m}^{\prime}\right)$ of $K_{m}^{\prime}$, and let $K_{m, P}$ be the preimage of $\bar{P}$ in $K_{m}$ under the map from $K_{m}$ to $\bar{G}_{m}$. Then, just as in Section 8 we have representations $\tilde{\kappa}_{m}^{\prime}$ of $K_{m}^{\prime}, \tilde{\kappa}_{m}^{\prime \prime}$ of $K_{m}^{\prime \prime}$, and $\tilde{\kappa}_{m, P}$ of $K_{m, P}$, satisfying:

$$
\begin{gathered}
\tilde{\kappa}_{m, P}^{\prime}=\left.\left(\tilde{\kappa}_{m}^{\prime}\right)\right|_{K_{m, P}} \\
\operatorname{Ind}_{K_{m}^{\prime \prime}}^{K_{m}^{\prime}} \tilde{\kappa}_{m}^{\prime \prime} \cong \tilde{\kappa}_{m}^{\prime} \\
\operatorname{Ind}_{K_{m, P}^{(\mathfrak{A})^{\times}}}^{\left(\mathfrak{\kappa}_{m, P}^{\prime}\right.} \cong \operatorname{Ind}_{K_{m}^{\prime}}^{\left(\mathfrak{A}^{\prime}\right)^{\times}} \tilde{\kappa}_{m}^{\prime} .
\end{gathered}
$$

Moreover, the intersection $K_{M}$ of $K_{m}^{\prime \prime}$ with $M$ is (under the identification of $M$ with a product of $j$ copies of $G_{m^{\prime}}$ ) equal to the product of $j$ copies of $K_{m^{\prime}}$. The restriction $\tilde{\kappa}_{M}$ of $\tilde{\kappa}_{m}^{\prime \prime}$ to $K_{M}$ factors as a product of $j$ copies of a representation $\tilde{\kappa}_{m^{\prime}}^{\prime}$ containing a character attached to $\mathfrak{A}_{m}$ via the endo-class $(\Theta, 0, \beta)$. Thus $\tilde{\kappa}_{m^{\prime}}^{\prime}$ differs from $\tilde{\kappa}_{m^{\prime}}$ by a twist.

We henceforth assume that, for each $m$, we have chosen $\tilde{\kappa}_{m}$ so that $\tilde{\kappa}_{m^{\prime}}^{\prime}$ is equal to $\tilde{\kappa}_{m^{\prime}}$. It is clear this can be done; the choice of $\kappa_{1}$ is arbitrary, and for each pair $m^{\prime}, m$ of successive elements of $\left\{1, e_{q f^{\prime}}, \ell e_{q^{f^{\prime}}}, \ldots\right\}$, changing $\tilde{\kappa}_{m}$ by twist changes $\tilde{\kappa}_{m^{\prime}}^{\prime}$ by the 'same' twist. With this assumption the representations $\tilde{\kappa}_{m}$, depend only on our choice of $\tilde{\kappa}_{1}$.

Finally, let $\mathcal{P}_{\bar{M}}$ denote the inflation of $\mathcal{P}_{\sigma_{m^{\prime}}}^{\otimes j}$ from $\bar{M}$ to a representation of $K_{M}$, and also (somewhat abusively) the inflation of $\mathcal{P}_{\sigma_{m^{\prime}}}^{\otimes j}$ to a representation of $K_{m}^{\prime \prime}$ (via the surjection of $K_{m}^{\prime \prime}$ onto $\bar{P}$ ).

Exactly as in Section 8, we obtain isomorphisms:

$$
\begin{gathered}
\mathrm{c}-\operatorname{Ind}_{K_{m}^{\prime \prime}}^{G_{m}} \tilde{\kappa}_{m}^{\prime \prime} \otimes \mathcal{P}_{\bar{M}} \cong \mathrm{c}-\operatorname{Ind}_{K_{m, P}}^{G_{m}} \tilde{\kappa}_{m, P} \otimes \mathcal{P}_{\bar{M}} \cong \mathrm{c}-\operatorname{Ind}_{K_{m}}^{G_{m}} \tilde{\kappa}_{m} \otimes i \bar{G}_{\bar{P}} \mathcal{P}_{\bar{M}} \\
H\left(G, K_{m}^{\prime \prime}, \tilde{\kappa}_{m}^{\prime \prime} \otimes \mathcal{P}_{\bar{M}}\right) \cong H\left(G_{m}, K_{P}, \tilde{\kappa}_{m, P} \otimes \mathcal{P}_{\bar{M}}\right) \cong H\left(G_{m}, K_{m}, \tilde{\kappa}_{m} \otimes i \bar{G}_{\bar{P}} \mathcal{P}_{\bar{M}}\right)
\end{gathered}
$$


Moreover, the discussion in Section 8 following Theorem 8.2 shows that these maps are compatible with supports.

We are now in a position to prove an analogue of Theorem 7.3:

THEOREM 9.5. The pair $\left(K_{m}^{\prime \prime}, \tilde{\kappa}_{m}^{\prime \prime} \otimes \mathcal{P}_{\bar{M}}\right)$ is a $G$-cover of $\left(K_{M}, \tilde{\kappa}_{M} \otimes \mathcal{P}_{\bar{M}}\right)$.

Proof. By Lemma 7.7, it suffices to construct a sequence of central elements $\lambda_{1}, \ldots, \lambda_{r}$ of $M$, whose product is strictly positive, and for each $i$ an invertible element $x_{i}$ of $H\left(G_{m}, K_{m}^{\prime \prime}, \tilde{\kappa}^{\prime \prime} \otimes \mathcal{P}_{\bar{M}}\right)$ supported on $K_{m}^{\prime \prime} \lambda_{i}^{\prime} K_{m}^{\prime \prime}$, such that $T^{+} x_{i}$ is invertible.

Let $w$ be an element of $W\left(G_{m}\right)$ that maps $V_{m^{\prime}, 1}$ to $V_{m^{\prime}, j}$, and $V_{m^{\prime}, i}$ to $V_{m^{\prime}, i-1}$ for $1<i \leqslant j$, and let $z$ be the central element of $M$ that acts by multiplication by $\varpi_{E}$ on $V_{m^{\prime}, 1}$ and by the identity on all other $V_{m^{\prime}, i}$. Let $\Pi=w z$. Then $\Pi$ normalizes $K_{m}^{\prime \prime}$, and intertwines $\tilde{\kappa}_{m}^{\prime \prime}$. Let $\alpha$ be any isomorphism $\tilde{\kappa}_{m}^{\prime \prime} \cong\left(\tilde{\kappa}_{m}^{\prime \prime}\right)^{\Pi}$, and let $\beta$ be the automorphism of $\mathcal{P}_{\bar{M}}=\mathcal{P}_{m^{\prime}}^{\otimes j}$ that cyclically permutes the tensor factors. Then $\alpha \otimes \beta$ is an isomorphism of $\tilde{\kappa}_{m}^{\prime \prime} \otimes \mathcal{P}_{\bar{M}}$ with its $\Pi$-conjugate. Let $y$ be the unique element of $H\left(G_{m}, K_{m}^{\prime \prime}, \tilde{\kappa}_{m}^{\prime \prime} \otimes \mathcal{P}_{\bar{M}}\right)$ supported on $K_{m}^{\prime \prime} \Pi K_{m}^{\prime \prime}$ that takes the value $\alpha \otimes \beta$ at $\Pi$. Since $\Pi$ normalizes $K_{m}^{\prime \prime}$, and $\alpha \otimes \beta$ is an isomorphism, left multiplication by $y$ induces an isomorphism:

$$
H\left(G_{m}, K_{m}^{\prime \prime}, \tilde{\kappa}_{m}^{\prime \prime} \otimes \mathcal{P}_{\bar{M}}\right)_{K_{m}^{\prime \prime} x K_{m}^{\prime \prime}} \rightarrow H\left(G_{m}, K_{m}^{\prime \prime}, \tilde{\kappa}_{m}^{\prime \prime} \otimes \mathcal{P}_{\bar{M}}\right)_{K_{m}^{\prime \prime} \Pi x K_{m}^{\prime \prime}}
$$

for all $x$, and an analogous statement holds for right multiplication.

Now for $1 \leqslant i \leqslant j$, let $\lambda_{i}=w^{-i} \Pi^{i}$. Then $\lambda_{i}$ is a central and positive (but not strictly positive) element of $M$, and the product of the $\lambda_{i}$ is strictly positive. Let $x_{i}$ be the element of $H\left(M, K_{M}, \tilde{\kappa}_{M} \otimes \mathcal{P}_{\bar{M}}\right)$ giving the action of $\lambda_{i}$ on c-Ind $K_{M}^{M} \tilde{\kappa}_{M} \otimes$ $\mathcal{P}_{\bar{M}}$. It suffices to show $T^{+} x_{i}$ is invertible for each $i$; equivalently, it suffices to show that $\left(T^{+} x_{i}\right) y^{-i}$ is an invertible element $v_{i}$ of $H\left(G_{m}, K_{m}^{\prime \prime}, \tilde{\kappa}_{m}^{\prime \prime} \otimes \mathcal{P}_{\bar{M}}\right)$.

Since $v_{i}$ is supported on $K_{m}^{\prime \prime} w^{-i} K_{m}^{\prime \prime}$, and the subalgebra of

$$
H\left(G_{m}, K_{m}^{\prime \prime}, \tilde{\kappa}_{m}^{\prime \prime} \otimes \mathcal{P}_{\bar{M}}\right)
$$

supported on $K_{m}$ is isomorphic to the endomorphism ring of $i \bar{P}_{m} \mathcal{P}_{\bar{M}}$, we may interpret $v_{i}$ as being induced from an endomorphism $\bar{v}_{i}$ of $i_{\bar{P}_{m}} \mathcal{P}_{\bar{M}}$. Exactly as in Section 7, one verifies that $\bar{v}_{i}$ is the automorphism of this space described in [HL, Theorem 2.4], completing the argument.

Our construction of the $\Theta_{i, m}$ now proceeds in a manner similar to that of Section 7. In particular, we obtain a map:

$$
E_{m^{\prime}}^{\otimes j} \rightarrow H\left(G_{m}, K_{m}^{\prime \prime}, \tilde{\kappa}_{m}^{\prime \prime} \otimes \mathcal{P}_{\bar{M}}\right) \cong H\left(G_{m}, K_{m}, \tilde{\kappa}_{m} \otimes i \bar{G}_{\bar{P}} \mathcal{P}_{\bar{M}}\right)
$$


Recall that $\mathcal{P}_{m}^{\prime}$ is the image of $i \frac{\bar{G}_{m}}{P}$ in $\mathcal{P}_{m}$ under the natural map from the former to the latter. Exactly as in Section 7, we have:

PROPOSITION 9.6. There is a surjection:

$$
H\left(G_{m}, K_{m}, \tilde{\kappa}_{m} \otimes \mathcal{P}_{m}\right) \rightarrow H\left(G_{m}, K_{m}, \tilde{\kappa}_{m} \otimes \mathcal{P}_{m}^{\prime}\right)
$$

compatible with the inclusion of $\mathrm{c}-\operatorname{Ind}_{K_{m}}^{G_{m}} \tilde{\kappa}_{m} \otimes \mathcal{P}_{m}$ in $\mathrm{c}-\operatorname{Ind}_{K_{m}}^{G_{m}} \tilde{\kappa}_{m} \otimes \mathcal{P}_{m}^{\prime}$ and the actions of the respective Hecke operators on these spaces. Moreover, this surjection is an isomorphism away from double cosets of the form $K_{m} z_{m, m}^{r} K_{m}$.

Proof. The proof is identical to that of Proposition 7.5, except that elements of the form $z_{m, m}^{r}$ are no longer central, so the statement must be rephrased slightly.

Similarly, in a manner identical to the proof of Lemma 7.6, we have:

LEMMA 9.7. Let $c$ be a central element of $H\left(G_{m}, K_{m}, \tilde{\kappa}_{m} \otimes i{\overline{G_{m}}}_{\bar{P}} \mathcal{P}_{\bar{M}}\right)$. Then $c$ preserves the kernel of the surjection:

$$
\mathrm{c}-\operatorname{Ind}_{K_{m}}^{G_{m}} \tilde{\kappa}_{m} \otimes i \overline{\bar{P}}_{\bar{P}}^{\bar{G}_{m}} \mathcal{P}_{\bar{M}} \rightarrow \mathrm{c}-\operatorname{Ind}_{K_{m}}^{G_{m}} \tilde{\kappa}_{m} \otimes \mathcal{P}_{\sigma_{m}}^{\prime},
$$

and thus descends to an element of $H\left(G_{m}, K_{m}, \tilde{\kappa}_{m} \otimes \mathcal{P}_{\sigma_{m}}^{\prime}\right)$.

With these results in hand we return to the inductive construction. Fix $m^{\prime}$ immediately preceding $m$, and suppose that we have constructed elements $\Theta_{i, m^{\prime}}$ as in Theorem 9.4. Suppose further that for $1 \leqslant i<m^{\prime}$, the element $\Theta_{i, m^{\prime}}$ is supported away from double cosets of the form $K_{m^{\prime}} z_{m^{\prime}, m^{\prime}}^{r} K_{m^{\prime}}$, and that $\Theta_{m^{\prime}, m^{\prime}}$ is an element of $E_{K_{m^{\prime}}, \tau_{m^{\prime}}}$ supported on $K_{m^{\prime}} z_{m, m} K_{m^{\prime}}$, and is of the form $\phi \otimes 1$, where $\phi$ lies in $I_{z_{m^{\prime}, m^{\prime}}}\left(\tilde{\kappa}_{m^{\prime}}\right)$. (These stipulations hold when $m^{\prime}=1$ by construction, and we will show that the inductive construction of the $\Theta_{i, m^{\prime}}$ implies these conditions for each larger $m^{\prime}$ as well.) We now turn to constructing the elements $\Theta_{i, m}$.

As in Section 7, we first define $\tilde{\Theta}_{i, m}$ be the element of $E_{m^{\prime}}^{\otimes j}$ defined by the formula:

$$
\tilde{\Theta}_{i, m}=\sum_{r_{1}+\cdots+r_{j}=i} \Theta_{r_{1}, m^{\prime}} \otimes \cdots \otimes \Theta_{r_{j}, m^{\prime}},
$$

and let $\bar{\Theta}_{i, m}$ be the image of $\tilde{\Theta}_{i, m}$ in $H\left(G_{m}, K_{m}, \tilde{\kappa}_{m} \otimes i \bar{G}_{m} \mathcal{P}_{\bar{M}}\right)$. For $i<m$, $\bar{\Theta}_{i, m}$ is supported away from cosets of the form $K_{m} z_{m, m}^{r} K_{m}$. By contrast, $\bar{\Theta}_{m, m}$ is supported on $K_{m} z_{m, m} K_{m}$, and has the form $\phi \otimes 1$ for an element $\phi$ of $I_{z_{m, m}}(\tilde{\kappa})$.

The arguments of Section 7 show that for any $s$ with $\ell$-regular part $\left(s_{1}^{\prime}\right)^{m}$, and any $s_{1}, \ldots, s_{j}$ in $\bar{G}_{m^{\prime}}$ such that the 'block' matrix with blocks $s_{1}, \ldots, s_{j}$ is 
conjugate to $s$, the action of $\bar{\Theta}_{i, m}$ on the summand c-Ind $K_{m}^{G_{m}} \tilde{\kappa}_{m} \otimes i \overline{\bar{G}}_{\bar{P}} \otimes_{r} \mathrm{St}_{s_{r}}$ of c-Ind $K_{m}^{G_{m}} \tilde{\kappa}_{m} \otimes i \frac{\bar{G}_{m}}{P} \mathcal{P}_{\bar{M}} \otimes \overline{\mathcal{K}}$ is via the element $\theta_{i, s}$ of $A_{M_{s}, \pi_{s}}$. In particular, $\bar{\Theta}_{i, m}$ is central in $H\left(G_{m}, K_{m}, \tilde{\kappa}_{m} \otimes i \bar{G}_{\bar{P}} \mathcal{P}_{\bar{M}}\right)$.

The construction of the elements $\Theta_{i, m}$ as in Theorem 9.4 is now straightforward. Consider the image of $\bar{\Theta}_{i, m}$ in $H\left(G_{m}, K_{m}, \tilde{\kappa}_{m} \otimes \mathcal{P}_{m}^{\prime}\right)$. For $i<m$, this image is supported away from cosets of the form $K_{m} z_{m, m}^{r} K_{m}$ and hence lifts uniquely to an element of $E_{m}$ supported away from $K_{m} z_{m, m}^{r} K_{m}$. Denote this element by $\Theta_{i, m}$; it is clear from our calculations that this has the claimed properties. For $i=m$, the element $\bar{\Theta}_{m, m}$ is supported on $K_{m} z_{m, m} K_{m}$ and has the form $\phi \otimes 1$ for some $\phi$ in $I_{z_{m, m}}(\tilde{\kappa})$; we let $\Theta_{m, m}$ denote the element $\phi \otimes 1$ of $H\left(G_{m}, K_{m}, \tilde{\kappa}_{m} \otimes \mathcal{P}_{m}\right)_{K_{m} z_{m, m} K_{m}}$. Again, it is clear that this element maps to $\theta_{m, s}$ for all $s$.

The proof of Theorem 9.4 is thus complete.

We now turn to considering the implications of Theorem 9.4. Our construction of the $\Theta_{i, m}$ gives us control over the mod $\ell$ cuspidal supports of the pairs $\left(M_{s}, \pi_{s}\right)$.

\section{THEOREM 9.8.}

(1) Let $V$ be an irreducible cuspidal representation of $G_{m}$ over $k$ containing $\left(K_{m}, \tau_{m}\right)$. Then the supercuspidal support of $V$ is inertially equivalent to $\left(L_{m}, \pi_{1}^{\otimes m}\right)$.

(2) For each semisimple conjugacy class $s$ in $\bar{G}_{m}$ with $\ell$-regular part $\left(s_{1}^{\prime}\right)^{m}$, the supercuspidal $\overline{\mathcal{K}}$-representation $\pi_{s}$ of $M_{s}$ has mod $\ell$ inertial supercuspidal support equivalent to $\left(L_{m}, \pi_{1}^{\otimes m}\right)$.

(3) Let $V$ be an irreducible cuspidal representation of $G_{r}$ over $k$ whose mod $\ell$ inertial supercuspidal support is equivalent to $\left(L_{r}, \pi_{1}^{\otimes r}\right)$. Then $r$ lies in $\left\{1, e_{q^{f^{\prime}}}, \ell e_{q^{f^{\prime}}}, \ldots\right\}$, and $V$ contains $\left(K_{r}, \tau_{r}\right)$.

(4) Let $V$ be an irreducible supercuspidal representation of $G_{r}$ over $\overline{\mathcal{K}}$ whose mod $\ell$ inertial supercuspidal support is equivalent to $\left(L_{r}, \pi_{1}^{\otimes r}\right)$. Then $r$ lies in $\left\{1, e_{q^{f^{\prime}}}, \ell e_{q^{f^{\prime}}}, \ldots\right\}$, and $V$ is an unramified twist of $\pi_{s}$ for some irreducible semisimple conjugacy class $s$ in $\bar{G}_{r}$ with $\ell$-regular part $\left(s_{1}^{\prime}\right)^{r}$.

Proof. These claims all follow easily from results of Vigneras in [V2]. However, they also follow directly from what we have proven above. For completeness, and with an eye towards potential generalizations to situations not covered by [V2], we give this alternative argument:

For the first claim, note that we have a surjection $\mathcal{P}_{K_{m}, \tau_{m}} \rightarrow V$. Let $\tilde{V}$ be a cuspidal lift of $V$ to a representation over $\bar{K}$; since $\tilde{V}$ admits a nonzero map from 
$\mathcal{P}_{K_{m}, \tau_{m}}, \tilde{V}$ must lie in $\operatorname{Rep}_{\bar{K}}(G)_{M_{s}, \pi_{s}}$ as above. Moreover, since $\tilde{V}$ is cuspidal we must have $M_{s}=G$ and $\pi_{s}$ cuspidal; in this case $\tilde{V}$ is an unramified twist of $\pi_{s}$. We thus have an action of $E_{K_{m}, \tau_{m}}$ on $\tilde{V}$ via the maps:

$$
E_{K_{m}, \tau_{m}} \rightarrow A_{M_{s}, \pi_{s}} \rightarrow \operatorname{End}_{\bar{K}[G]}(\tilde{V}) \cong \bar{K}
$$

This action preserves the image of $\mathcal{P}_{K_{m}, \tau_{m}}$ in $\tilde{V}$ and thus reduces modulo $\ell$ to a map $\alpha: E_{K_{m}, \tau_{m}} \rightarrow k$. If we let $E_{K_{m}, \tau_{m}}$ act on $V$ via $\alpha$, then this action is compatible with the surjection of $\mathcal{P}_{K_{m}, \tau_{m}}$ onto $V$.

Let $\mathfrak{m}$ be the kernel of $\alpha$, and let $V^{\prime}$ be the quotient $\mathcal{P}_{K_{m}, \tau_{m}} / \mathfrak{m} \mathcal{P}_{K_{m}, \tau_{m}}$. Every endomorphism of a quotient of $V^{\prime}$ lifts to an element of $E_{K_{m}, \tau_{m}}$, and is thus a scalar; this implies that the cosocle of $V^{\prime}$ is absolutely irreducible. Since we have a surjection $V^{\prime} \rightarrow V$, the cosocle of $V^{\prime}$ is isomorphic to $V$.

On the other hand, we have a map $E_{K_{m}, \tau_{m}} \rightarrow W(k)\left[Z_{0, m}\right]^{W_{M_{0, m}}\left(\left(s_{1}^{\prime}\right)^{m}\right)}$ giving the action of $E_{K_{m}, \tau_{m}}$ on c- $\operatorname{Ind}_{K_{m}}^{G_{m}} \tilde{\kappa}_{m} \otimes \mathrm{St}_{\left(s_{1}^{\prime}\right)^{m}}$, and the target of this map is generated by the images of the $\Theta_{i, m}$. It is then straightforward to verify that the map $\alpha$ coincides with the composition:

$$
E_{K_{m}, \tau_{m}} \rightarrow W(k)\left[Z_{0, m}\right]^{W_{M_{0, m}}\left(\left(s_{1}^{\prime}\right)^{m}\right)} \rightarrow k
$$

where the second map takes the images of $\Theta_{1, m}, \ldots, \Theta_{m-1, m}$ to zero and takes the image of $\Theta_{m, m}$ to $\alpha\left(\Theta_{m, m}\right)$. (This amounts to verifying that $\Theta_{i, m}$ acts by zero on $V$ for $1 \leqslant i \leqslant m-1$, but this is clear since $\tilde{V}$ is cuspidal: the action on $V$ thus factors through the action of $E_{K_{m}, \tau_{m}}$ on $\tilde{V}$ and for $1 \leqslant i \leqslant m-1$, the elements $\Theta_{i, m}$ annihilate the cuspidals.)

Let $W$ be the image of $\mathcal{P}_{K_{m}, \tau_{m}}$ in c-Ind ${ }_{K_{m}}^{G_{m}} \tilde{\kappa}_{m} \otimes \mathrm{St}_{\left(s_{1}^{\prime}\right)^{m}}$. We have a surjection $V^{\prime} \rightarrow W / \mathfrak{m} W$, and hence in particular $V$ is isomorphic to a subquotient of $W$. On the other hand, since $W \otimes \overline{\mathcal{K}}$ is isomorphic to c- $\operatorname{Ind}_{K_{m}}^{G_{m}} \tilde{\kappa}_{m} \otimes \operatorname{St}_{\left(s_{1}^{\prime}\right)^{m}}$, it follows that $W \otimes \overline{\mathcal{K}}$ lies in $\operatorname{Rep}_{\overline{\mathcal{K}}}(G)_{M_{\left(s_{1}^{\prime}\right)}, \pi_{\left(s_{1}^{\prime}\right)^{m}}}$, and thus that every subquotient of $W$ has $\bmod \ell$ inertial supercuspidal support $\left(L_{m}, \pi_{1}^{\otimes m}\right)$.

For the second claim, note that it suffices to prove this when $s$ is irreducible, and in this case $\pi_{s}$ contains $\left(K_{m}, \tilde{\kappa} \otimes \mathrm{St}_{s}\right)$. Twisting so that $\pi_{s}$ is integral, we find that the $\bmod \ell$ reduction of $\pi_{s}$ contains $\left(K_{m}, \tau_{m}\right)$ and so we are done by claim (1).

Now let $V$ be an irreducible cuspidal representation of $G_{r}$ over $k$ for some $r$. Then $V$ contains a maximal distinguished cuspidal $k$-type $\left(K^{\prime}, \tau^{\prime}\right)$. Write $\tau^{\prime}=\kappa^{\prime} \otimes \sigma^{\prime}$. Then $\sigma^{\prime}$ is attached to an $\ell$-regular semisimple conjugacy class $t$ in $\bar{G}_{r}$; moreover, as $\sigma^{\prime}$ is cuspidal, $t$ is the $\ell$-regular part of an irreducible conjugacy class and thus has the form $\left(t^{\prime}\right)^{r^{\prime}}$ for some irreducible $\ell$-regular conjugacy class $t^{\prime}$ and some positive integer $r^{\prime}$ dividing $n_{1} r$. Let $n_{1}^{\prime}=n_{1} r / r^{\prime}$ and fix a maximal order in $\mathrm{GL}_{n_{1}^{\prime}}(F)$. Then the endo-class $\left(\Theta^{\prime}, 0, \beta^{\prime}\right)$ attached to $\left(K^{\prime}, \tau^{\prime}\right)$ associates to this maximal order a compact open subgroup $K_{1}^{\prime}$ of $\mathrm{GL}_{n_{1}^{\prime}}(F)$ and 
a representation $\kappa_{1}$ of $K_{1}^{\prime}$ (defined up to twist). Set $\tau_{1}^{\prime}=\kappa_{1}^{\prime} \otimes \sigma_{t^{\prime}}$, where $\sigma_{t^{\prime}}$ is the supercuspidal representation associated to $t^{\prime}$; then $\left(K_{1}^{\prime}, \tau_{1}^{\prime}\right)$ is a maximal distinguished supercuspidal type, and the construction of this section associated to this type a family $\left(K_{m}^{\prime}, \tau_{m}^{\prime}\right)$ for suitable $m$. Moreover, we may choose the twist of $\kappa_{1}$ so that $\left(K^{\prime}, \tau^{\prime}\right)$ is equivalent to the type $\left(K_{r}^{\prime}, \tau_{r}^{\prime}\right)$. Then claim (1) above shows that $V$ has supercuspidal support inertially equivalent to $\left(\pi^{\prime}\right)^{\otimes r^{\prime}}$, where $\pi^{\prime}$ is some supercuspidal $k$-representation of $\mathrm{GL}_{n_{1}^{\prime}}(F)$ containing $\left(K_{1}^{\prime}, \tau_{1}^{\prime}\right)$. But then $r=r^{\prime}$ and $\pi^{\prime}$ and $\pi$ are unramified twists of each other, so $\left(K_{1}^{\prime}, \tau_{1}^{\prime}\right)$ is equivalent to $\left(K_{1}, \tau_{1}\right)$. It now follows that $\sigma_{t^{\prime}}$ is a unramified twist of $\sigma_{1}$ by a character, so that $s^{\prime}$ and $t^{\prime}$ differ by an element of the center of $\bar{G}_{1}$. Thus $r$ must lie in $\left\{1, e_{q^{f^{\prime}}}, \ell e_{q^{f^{\prime}}}, \ldots\right\}$ so the type $\left(K_{r}, \tau_{r}\right)$ makes sense and is equivalent to $\left(K^{\prime}, \tau^{\prime}\right)$. Thus $V$ contains $\left(K^{\prime}, \tau^{\prime}\right)$ as claimed.

Claim (4) follows from (3) easily by reducing an unramified twist of $V$ modulo $\ell$.

We conclude this section by giving a complete description of $E_{K_{m}, \tau_{m}}$ for small $m$; that is, for $m<\ell$. There are two cases to consider; in the first, $m=1$, and in the second $m=e_{q^{f^{\prime}}}>1$.

When $m=1$, the element $\Theta_{1,1}^{r}$ of $E_{K_{1}, \tau_{1}}$ generates $\left(E_{K_{1}, \tau_{1}}\right)_{K_{1} z_{1,1}^{r} K_{1}}$ as an $E_{\sigma_{1}}$ module for all $r$, and hence we have

$$
E_{K_{1}, \tau_{1}}=E_{\sigma_{1}}\left[\Theta_{1,1}^{ \pm 1}\right] .
$$

This case was already studied by Dat in [D4]; in particular Dat shows that $E_{\sigma_{1}}$ is a universal deformation ring of $\sigma_{1}$, and that, after completing at any maximal ideal of characteristic $\ell, E_{K_{1}, \tau_{1}}$ becomes the universal deformation ring of the corresponding supercuspidal representation.

When $m>1$ but $m<\ell$, then any $s \neq\left(s_{1}^{\prime}\right)^{m}$ with $s^{\text {reg }}=\left(s_{1}^{\prime}\right)^{m}$ is irreducible. We have an ideal $I^{\text {cusp }}$ of $E_{\sigma_{m}}$ that is the kernel of the action of $E_{\sigma_{m}}$ on $\operatorname{St}_{\left(s_{1}^{\prime}\right)^{m}}$.

Proposition 9.9. When $1<m<\ell$, we have an isomorphism:

$$
E_{K_{m}, \tau_{m}} \cong E_{\sigma_{m}}\left[\Theta_{1, m}, \ldots, \Theta_{m, m}^{ \pm 1}\right] /\left\langle\Theta_{1, m}, \ldots, \Theta_{m-1, m}\right\rangle \cdot I^{\text {cusp }} .
$$

Proof. As $E_{\sigma_{m}}$ and the $\Theta_{i, m}$ are contained in $E_{K_{m}, \tau_{m}}$, we have a map:

$$
E_{\sigma_{m}}\left[\Theta_{1, m}, \ldots, \Theta_{m, m}^{ \pm 1}\right] \rightarrow E_{K_{m}, \tau_{m}} .
$$

It is easy to see that $\left\langle\Theta_{1, m}, \ldots, \Theta_{m-1, m}\right\rangle$ map to zero in $H\left(G_{m}, K_{m}, \tilde{\kappa}_{m} \otimes \mathrm{St}_{s}\right)$ for $s \neq\left(s_{1}^{\prime}\right)^{m}$, so that $\left\langle\Theta_{1, m} \ldots, \Theta_{m-1, m}\right\rangle \cdot I^{\text {cusp }}$ is in the kernel of the map to $E_{K_{m}, \tau_{m}}$. Denote this ideal by $J$.

To see that this ideal is precisely the kernel, first note that the restriction of this map to $E_{\sigma_{m}}\left[\Theta_{m, m}^{ \pm 1}\right]$ identifies $E_{\sigma_{m}}\left[\Theta_{m, m}^{ \pm 1}\right]$ with the subring of $E_{K_{m}, \tau_{m}}$ supported on 
elements of the form $K_{m} z_{m, m}^{r} K_{m}$. Moreover, the ideal of $E_{\sigma_{m}}\left[\Theta_{1, m}, \ldots, \Theta_{m, m}^{ \pm 1}\right] / J$ generated by the $\Theta_{i, m}$ for $1 \leqslant i \leqslant m-1$ is a complementary $W(k)$-submodule of the subring $E_{\sigma_{m}}\left[\Theta_{m, m}^{ \pm 1}\right]$. When restricted to the ideal generated by the elements $\Theta_{1, m}, \ldots, \Theta_{m-1, m}$, the composed map:

$$
E_{\sigma_{m}}\left[\Theta_{1, m}, \ldots, \Theta_{m, m}^{ \pm 1}\right] / J \rightarrow E_{K_{m}, \tau_{m}} \rightarrow H\left(G_{m}, K_{m}, \tilde{\kappa}_{m} \otimes \mathrm{St}_{\left(s_{1}^{\prime}\right)^{m}}\right)
$$

is injective, and its image is precisely the subring of $H\left(G, K, \tilde{\kappa}_{m} \otimes \mathrm{St}_{\left(s_{1}^{\prime}\right)^{m}}\right)$ supported away from elements of the form $K_{m} z_{m, m}^{r} K_{m}$. From this we see that the map

$$
E_{\sigma_{m}}\left[\Theta_{1, m}, \ldots, \Theta_{m, m}^{ \pm 1}\right] / J \rightarrow E_{K_{m}, \tau_{m}}
$$

is injective.

To see surjectivity, note that the map $E_{K_{m}, \tau_{m}} \rightarrow H\left(G_{m}, K_{m}, \tilde{\kappa}_{m} \otimes \mathrm{St}_{\left(s_{1}^{\prime}\right)^{m}}\right)$ is an isomorphism away from double cosets of the form $K_{m} z_{m, m}^{r} K_{m}$, so any element of $E_{K_{m}, \tau_{m}}$ supported away from the latter is in the image of the map from $E_{\sigma_{m}}\left[\Theta_{1, m}, \ldots, \Theta_{m, m}^{ \pm 1}\right]$. On the other hand we have already seen that every element supported on a double coset $K_{m} z_{m, m}^{r} K_{m}$ is in the image, so the result is proven.

REMARK 9.10. When $\ell>n$, and $\sigma$ is not supercuspidal, Paige gives an explicit description of $E_{\sigma}$ as a $W(k)$-algebra in [Pg, Theorem 4.11]. The above proposition thus gives a complete description of $E_{K, \tau}$ in this case.

\section{Finiteness results}

Our next goal is to establish fundamental finiteness results for $\mathcal{P}_{K, \tau}$. In order to do so it will be necessary to work integrally with lattices inside a generic pseudotype $\left(K, \tilde{\kappa} \otimes \mathrm{St}_{s}\right)$. Choose a finite extension $\mathcal{K}^{\prime}$ of $\mathcal{K}$ such that $\mathrm{St}_{s}$ is defined over $\mathcal{K}^{\prime}$, and let $\mathcal{O}^{\prime}$ be the ring of integers in $\mathcal{K}^{\prime}$. We can then consider $\mathcal{O}^{\prime}$-lattices $L_{s}$ inside $\mathrm{St}_{s}$, and consider the 'integral generic pseudotype' $\left(K, \tilde{\kappa} \otimes L_{s}\right)$, and try to determine the structure of $\mathrm{c}-\operatorname{Ind}_{K}^{G} \tilde{\kappa} \otimes L$ as a module over $H\left(G, K, \tilde{\kappa} \otimes L_{s}\right)$.

There will be two lattices in $\mathrm{St}_{s}$ of particular interest to us. We construct the first of these as follows: denote by $\bar{M}$ the Levi subgroup $\bar{M}_{s}$ of $\bar{G}$. The representation $\mathrm{St}_{\bar{M}, s}$ is irreducible, cuspidal and defined over $\mathcal{K}^{\prime}$, and remains irreducible when reduced $\bmod \ell$. There is thus a $\bar{M}$-stable $\mathcal{O}^{\prime}$-lattice $L_{\bar{M}}$ in $\mathrm{St}_{\bar{M}, s}$, and such an $L_{\bar{M}}$ is unique up to homothety. Then $i \frac{\bar{G}}{P} L_{\bar{M}, s}$ is an $\mathcal{O}^{\prime}$-lattice in $I_{s}$. Let $L_{s}$ be the image of this lattice in $\mathrm{St}_{s}$.

The second lattice we will make use of will be denoted $L_{s}^{\prime}$, and is defined as follows: the representation $\mathrm{St}_{s}$ is a direct summand of $\mathcal{P}_{\sigma} \otimes_{W(k)} \mathcal{K}^{\prime}$. Let $L_{s}^{\prime}$ be the image of $\mathcal{P}_{\sigma} \otimes_{W(k)} \mathcal{O}^{\prime}$ under the projection to $\mathrm{St}_{s}$; this defines $L_{s}^{\prime}$ up to homothety. The lattice $L_{s}^{\prime}$ is the one that is of interest to us in applications, but 
is more complicated; we will study it via its relationship with $L_{s}$. Note that there exist $a, b$ such that $\ell^{a} L_{s} \subset L_{s}^{\prime} \subset \ell^{b} L_{s}$. Let $\tau_{L_{s}}$ and $\tau_{L_{s}^{\prime}}$ denote the representations $\tilde{\kappa} \otimes L_{s}$, and $\tilde{\kappa} \otimes L_{s}^{\prime}$.

The pair $\left(K, \tau_{L_{s}}\right)$ is not difficult to understand; indeed, the arguments of Section 8 apply. In particular, consider the pair $\left(K_{M}, \tilde{\kappa}_{M} \otimes L_{\bar{M}}\right)$, where $M, K_{M}$, and $\tilde{\kappa}_{M}$ are as in Section 8. It follows from Lemma 8.4 that $\left(K^{\prime \prime}, \tilde{\kappa}^{\prime \prime} \otimes L_{\bar{M}}\right)$ is a $G-$ cover of $\left(K_{M}, \tilde{\kappa}_{M} \otimes L_{\bar{M}}\right)$, and that the center of $H\left(G, K^{\prime \prime}, \tilde{\kappa}^{\prime \prime} \otimes L_{\bar{M}}\right)$ is isomorphic to $\mathcal{O}^{\prime}\left[Z_{S}\right]^{W_{\bar{M}}(s)}$. (Indeed, a choice of a compatible family of cuspidals for the tower of types that contains $(K, \tau)$ gives rise to explicit isomorphisms:

$$
\begin{gathered}
H\left(M, K_{M}, \tilde{\kappa}_{M} \otimes L_{\bar{M}}\right) \cong \mathcal{O}^{\prime}\left[Z_{s}\right] \\
Z\left(H\left(G, K^{\prime \prime}, \tilde{\kappa}^{\prime \prime} \otimes L_{\bar{M}}\right)\right) \cong \mathcal{O}^{\prime}\left[Z_{s}\right]^{W_{\bar{M}}(s)} .
\end{gathered}
$$

Henceforth we fix such a choice.) The intertwining calculations of Section 8 give rise to a support-preserving isomorphism $H\left(G, K^{\prime \prime}, \tilde{\kappa}^{\prime \prime} \otimes L_{\bar{M}}\right)$ with $H\left(G, K_{\bar{P}}, \tilde{\kappa}_{\bar{P}} \otimes L_{\bar{M}}\right)$, and an isomorphism of the latter with $H\left(G, K, \tilde{\kappa} \otimes i_{\bar{P}}^{\bar{G}} L_{\bar{M}}\right)$.

We now observe:

LEMma 10.1. Let $x$ be a central element of $H\left(G, K, \tilde{\kappa} \otimes i \overline{\bar{G}} L_{\bar{M}}\right)$. Then $x$ descends to an endomorphism of $\mathrm{c}-\operatorname{Ind}_{K}^{G} \tilde{\kappa} \otimes L_{s}$ via the surjection of $i \frac{\bar{G}}{P} L_{\bar{M}}$ onto $L_{s}$.

Proof. The results of Section 8, particularly the discussion after the proof of Proposition 8.7, show that this holds after inverting $\ell$. We have a surjection:

$$
\mathrm{c}-\operatorname{Ind}_{K}^{G} \tilde{\kappa} \otimes\left(i \overline{\bar{G}} L_{\bar{M}}\right) \rightarrow \mathrm{c}-\operatorname{Ind}_{K}^{G} \tilde{\kappa} \otimes L_{s},
$$

and it suffices to show that $x$ preserves the kernel of this surjection. But as this holds after inverting $\ell$, and both the left-hand and right-hand sides are $\ell$-torsion free, the result follows.

We next turn to questions of admissibility:

LEMMA 10.2. The module c-Ind $K_{M}^{M} \tilde{\kappa} \otimes L_{M}$ is an admissible $H\left(M, K_{M}, \tilde{\kappa}_{M} \otimes L_{M}\right)$ module.

Proof. Let $\pi$ be an $\mathcal{O}^{\prime}[M]$-module such that $\pi \otimes_{\mathcal{O}^{\prime}} \mathcal{K}^{\prime}$ is absolutely irreducible and such that the restriction of $\pi$ to $K_{M}$ admits a nonzero map from $\tilde{\kappa} \otimes L_{M}$. Then $\operatorname{Hom}_{\mathcal{O}^{\prime}\left[K_{M}\right]}\left(\tilde{\kappa} \otimes L_{M}, \pi\right)$ is a free $\mathcal{O}^{\prime}$-module of rank one. Consider the $\mathcal{O}^{\prime}[M]$-module $\pi \otimes_{\mathcal{O}^{\prime}} \mathcal{O}^{\prime}\left[M / M_{0}\right]$, on which $M$ acts on $\mathcal{O}^{\prime}\left[M / M_{0}\right]$ via the natural character $M \rightarrow \mathcal{O}^{\prime}\left[M / M_{0}\right]$. We have

$$
\operatorname{Hom}_{\mathcal{O}^{\prime}\left[K_{M}\right]}\left(\tilde{\kappa} \otimes L_{M}, \pi\right) \cong \mathcal{O}^{\prime}\left[M / M_{0}\right]
$$


and $H\left(M, K_{M}, \tau\right)=\mathcal{O}^{\prime}[Z]$ acts on the right-hand side via the inclusion of $\mathcal{O}^{\prime}[Z]$ in $\mathcal{O}^{\prime}\left[M / M_{0}\right]$. This yields an isomorphism:

$$
\left(\mathrm{c}-\operatorname{Ind}_{K_{M}}^{M} \tilde{\kappa} \otimes L_{M}\right) \otimes_{\mathcal{O}^{\prime}\left[Z_{s}\right]} \mathcal{O}^{\prime}\left[M / M_{0}\right] \cong \pi \otimes_{\mathcal{O}^{\prime}} \mathcal{O}^{\prime}\left[M / M_{0}\right] .
$$

In particular, the left-hand side is admissible over $\mathcal{O}^{\prime}\left[M / M_{0}\right]$, and so c-Ind ${ }_{K_{M}}^{M} \tilde{\kappa} \otimes$ $L_{M}$ is admissible over $\mathcal{O}^{\prime}\left[Z_{s}\right]$.

LEMMA 10.3. Let $R$ be commutative $W(k)$-algebra, let $P=M U$ be a parabolic subgroup of $G$, and let $\pi$ be an admissible $R[M]$-module such that for any parabolic subgroup $P^{\prime}=M^{\prime} U^{\prime}$ of $M, r_{M}^{P^{\prime}} \pi$ is admissible as an $R\left[M^{\prime}\right]$-module. Then $i_{P}^{G} \pi$ is an admissible $R[G]-m o d u l e$, and, for any parabolic subgroup $P^{\prime \prime}=M^{\prime \prime} U^{\prime \prime}$ of $G, r_{G}^{P^{\prime \prime}} i_{P}^{G} \pi$ is an admissible $R\left[M^{\prime \prime}\right]$-module.

Proof. This is an immediate consequence of Bernstein-Zelevinsky's filtration of of the composite functor $r_{G}^{P^{\prime \prime}} i_{P}^{G}[\mathrm{BZ}, 2.12]$ together with the fact that parabolic induction takes admissible representations to admissible representations.

Let $R$ be the center of $H\left(G, K, \tilde{\kappa} \otimes i \frac{\bar{G}}{P} L_{\bar{M}}\right)$. (We may also regard $R$ as the center of $H\left(G, K^{\prime \prime}, \tilde{\kappa}^{\prime \prime} \otimes L_{\bar{M}}\right)$.) By Lemma 10.1, the ring $R$ acts on c-Ind $K_{K}^{G} \tau_{L_{s}}$.

PROPOSITION 10.4. The module c-Ind ${ }_{K}^{G} \tau_{L_{s}}$ is admissible over R. More generally, for any parabolic $P^{\prime}=M^{\prime} U^{\prime}$ in $G$, the module $r_{G}^{P^{\prime}} \mathrm{c}-\operatorname{Ind}_{K}^{G} \tau_{L_{s}}$ is admissible over $R$.

Proof. We have shown that we may identify the center of $H\left(G, K^{\prime \prime}, \tilde{\kappa}^{\prime \prime} \otimes L_{\bar{M}}\right)$ with the ring $\mathcal{O}^{\prime}\left[Z_{s}\right]^{W_{\bar{M}}(s)}$. This ring acts on c-Ind ${ }_{K}^{G} \tau_{L_{s}}$ via its identification with $R$.

Consider the module $\tilde{\kappa}_{M} \otimes L_{\bar{M}}$; this is a lattice in a maximal distinguished cuspidal $M$-type. Thus the $W(k)[M]$-module c-Ind $K_{K_{M}}^{M} \tilde{\kappa}_{M} \otimes L_{\bar{M}}$ is admissible and cuspidal over the Hecke algebra $H\left(M, K_{M}, \tilde{\kappa}_{M} \otimes L_{\bar{M}}\right)$, and the latter is isomorphic to $\mathcal{O}^{\prime}\left[Z_{s}\right]$. It follows that $i_{P}^{G} \mathrm{c}-\operatorname{Ind}_{K_{M}}^{M} \tilde{\kappa}_{M} \otimes L_{\bar{M}}$ is an admissible $\mathcal{O}^{\prime}\left[Z_{s}\right]$-module, as is $r_{G}^{P^{\prime}} i_{P}^{G} \mathrm{c}-\operatorname{Ind}_{K_{M}}^{M} \tilde{\kappa}_{M} \otimes L_{\bar{M}}$ for any $P^{\prime}$.

The subalgebra $\mathcal{O}^{\prime}\left[Z_{s}\right]^{W_{\bar{M}}^{(s)}}$ of $\mathcal{O}^{\prime}\left[Z_{s}\right]$ acts on both $i_{P}^{G} \mathrm{c}-\operatorname{Ind}_{K_{M}}^{M} \tilde{\kappa}_{M} \otimes L_{\bar{M}}$ and on c-Ind $K_{K^{\prime \prime}}^{G} \tilde{\kappa}^{\prime \prime} \otimes L_{\bar{M}}$. In fact, any map between these two modules is $\mathcal{O}^{\prime}\left[Z_{s}\right]^{W_{\bar{M}}(s)}-$ equivariant. To see this, note that we can identify $\mathcal{O}^{\prime}\left[Z_{s}\right]^{W_{\bar{M}}(s)} \otimes_{\mathcal{O}^{\prime}} \overline{\mathcal{K}}$ with the center $A_{M_{s}, \pi_{s}}$ of $\operatorname{Rep}_{\overline{\mathcal{K}}}(G)_{M_{s}, \pi_{s}}$, and thus the action of $\mathcal{O}^{\prime}\left[Z_{s}\right]^{W_{\bar{M}}(s)}$ is compatible with every map between the two modules after inverting $\ell$. Since both modules are $\ell$-torsion free the claim follows.

As $\mathcal{O}^{\prime}\left[Z_{s}\right]$ is a finitely generated $\mathcal{O}^{\prime}\left[Z_{s}\right]^{W_{\bar{M}}(s)}$-module, it now suffices to produce an embedding of c-Ind $K^{\prime \prime} \tilde{\kappa}^{\prime \prime} \otimes L_{\bar{M}}$ in $i_{P}^{G} \mathrm{c}-\operatorname{Ind}_{K_{M}}^{M} \tilde{\kappa}_{M} \otimes L_{\bar{M}}$. 
We first consider this question over $\overline{\mathcal{K}}$. In this situation the two modules are actually isomorphic. To see this, note that after tensoring with $\overline{\mathcal{K}}, \tilde{\kappa}^{\prime \prime} \otimes L_{\bar{M}}$ becomes $\tilde{\kappa}^{\prime \prime} \otimes \mathrm{St}_{\bar{M}, s}$, which is, in the language of [BK3], a semisimple type. In particular, $\operatorname{Hom}_{K^{\prime \prime}}\left(\tilde{\kappa}^{\prime \prime} \otimes \mathrm{St}_{\bar{M}, s},-\right)$ yields an equivalence between the block of $\operatorname{Rep}_{\overline{\mathcal{K}}}(G)$ corresponding to the type $\tilde{\kappa}^{\prime \prime} \otimes \mathrm{St}_{\bar{M}, s}$ and the category of $H\left(G, K^{\prime \prime}\right.$, $\left.\tilde{\kappa}^{\prime \prime} \otimes \mathrm{St}_{\bar{M}, s}\right)$-modules. On the other hand, it is an easy consequence of Theorem 6.4 that the space

$$
\operatorname{Hom}_{K^{\prime \prime}}\left(\tilde{\kappa}^{\prime \prime} \otimes \mathrm{St}_{\bar{M}, s}, i_{P}^{G} \mathrm{c}-\operatorname{Ind}_{K_{M}}^{M} \tilde{\kappa}_{M} \otimes \mathrm{St}_{\bar{M}, s}\right)
$$

is a free $H\left(G, K^{\prime \prime}, \tilde{\kappa}^{\prime \prime} \otimes \mathrm{St}_{\bar{M}, s}\right)$-module of rank one. We may thus identify $\mathrm{c}-\operatorname{Ind}_{K^{\prime \prime}}^{G} \tilde{\kappa}^{\prime \prime} \otimes \mathrm{St}_{\bar{M}, s}$ with $i_{P}^{G} \mathrm{c}-\mathrm{Ind}_{K_{M}}^{M} \tilde{\kappa}_{M} \otimes L_{\bar{M}}$.

Descending from $\overline{\mathcal{K}}$ to $\mathcal{K}^{\prime}$, we find an isomorphism of c-Ind ${ }_{K^{\prime \prime}}^{G} \tilde{\kappa}^{\prime \prime} \otimes L_{\bar{M}} \otimes \mathcal{K}^{\prime}$ into $i_{P}^{G} \mathrm{c}-\operatorname{Ind}_{K_{M}}^{M} \tilde{\kappa}_{M} \otimes L_{\bar{M}} \otimes \mathcal{K}^{\prime}$. It suffices to show that, after multiplying by a sufficiently large power of $\ell$, such a map takes c-Ind $K_{K^{\prime \prime}}^{G} \tilde{\kappa}^{\prime \prime} \otimes L_{\bar{M}}$ into $i_{P}^{G} \mathrm{c}-\operatorname{Ind}_{K_{M}}^{M} \tilde{\kappa}_{M} \otimes L_{\bar{M}}$. But this is clear since $G$-maps from the former to the latter are, by Frobenius reciprocity, the same as $K^{\prime \prime}$-maps from $\tilde{\kappa}^{\prime \prime} \otimes L_{\bar{M}}$ into the latter, and $\tilde{\kappa}^{\prime \prime} \otimes L_{\bar{M}}$ is finitely generated as an $\mathcal{O}^{\prime}$-module.

We now compare c-Ind ${ }_{K}^{G} \tau_{L_{s}}$ and c-Ind ${ }_{K}^{G} \tau_{L_{s}^{\prime}}$. The inclusions $\ell^{a} L_{s} \subset L_{s}^{\prime} \subset \ell^{b} L$ give rise to inclusions: $\ell^{a} \mathrm{c}-\operatorname{Ind}_{K}^{G} \tau_{L_{s}} \subset \mathrm{c}-\operatorname{Ind}_{K}^{G} \tau_{L_{s}^{\prime}} \subset \ell^{b} \mathrm{c}-\operatorname{Ind}_{K}^{G} \tau_{L_{s}}$.

The endomorphism ring $E_{K, \tau}$ of $\mathcal{P}_{K, \tau}$ preserves the factor c-Ind ${ }_{K}^{G} \tilde{\kappa} \otimes \mathrm{St}_{s}$ of $\mathcal{P}_{K, \tau} \otimes \overline{\mathcal{K}}$, and hence preserves the image of $\mathcal{P}_{K, \tau} \otimes \mathcal{O}^{\prime}$ in $\mathrm{c}-\operatorname{Ind}_{K}^{G} \tilde{\kappa} \otimes \mathrm{St}_{s}$. This image is equal to c-Ind ${ }_{K}^{G} \tau_{L_{s}^{\prime}}$. In particular, we obtain a map of $E_{K, \tau} \otimes \mathcal{O}^{\prime}$ into $H\left(G, K, \tau_{L_{s}^{\prime}}\right)$.

For some $m$, we have $(K, \tau)=\left(K_{m}, \tau_{m}\right)$, where $K_{m}$ and $\tau_{m}$ are as in Section 9. Our choice of compatible family of cuspidals in Section 9 gives an isomorphism:

$$
H\left(G, K, \tilde{\kappa} \otimes \mathrm{St}_{s}\right) \cong \overline{\mathcal{K}}\left[Z_{s}\right]^{W_{\bar{M}_{s}}(s)} .
$$

The isomorphism of $R \otimes \overline{\mathcal{K}}$ with $H\left(G, K, \tilde{\kappa} \otimes \mathrm{St}_{s}\right)$ identifies the ring $R$ with the subalgebra $\mathcal{O}^{\prime}\left[Z_{s}\right]^{W_{\bar{M}_{s}}(s)}$. We also identify $H\left(G, K, \tau_{L_{s}^{\prime}}\right)$ with another, yet-to-bedetermined $\mathcal{O}^{\prime}$-subalgebra that contains $E_{K, \tau} \otimes \mathcal{O}^{\prime}$.

We observe that as a subalgebra of $\overline{\mathcal{K}}\left[Z_{s}\right]^{W_{\bar{M}_{s}}(s)}, H\left(G, K, \tau_{L_{s}^{\prime}}\right)$ contains the images of the elements $\Theta_{i, m}$ of $C_{K, \tau}$, as well as the image of $\Theta_{m, m}^{-1}$. We may identify these with the elements $\theta_{i, s}$ of $\overline{\mathcal{K}}\left[Z_{s}\right]^{W_{\bar{M}}(s)}$, and hence with elements of $R$. We then observe:

PROPOSITION 10.5. The algebra $W(k)\left[Z_{s}\right]^{W_{\bar{M}}(s)}$ is a finitely generated module over the subalgebra $W(k)\left[\theta_{1, s}, \ldots, \theta_{m, s}, \theta_{m, s}^{-1}\right]$ In particular, $R$ is a finitely generated module over this subalgebra. 
Proof. Let $s_{1}, \ldots, s_{r}$ be the irreducible constituents of $s$, and let $z_{1}, \ldots, z_{r}$ be the elements of $Z_{s}$ such that, when considered as an element of $\operatorname{GL}_{n / e f}(E), z_{i}$ is scalar with entries $\varpi_{E}$ on the block of $Z_{s}$ corresponding to $s_{i}$, and the identity on all other blocks. Let $d_{i}$ be the degree of $s_{i}$ over $\mathbb{F}_{q^{f}}$, and let $d$ be the degree of $s^{\prime}$ over $\mathbb{F}_{q^{f}}$, where $s^{\text {reg }}=\left(s^{\prime}\right)^{m}$. Then, by definition, we have:

$$
\theta_{i, s}=\sum_{S} \prod_{j \in S} z_{j}
$$

where $S$ runs over those subsets of $1, \ldots, r$ such that

$$
\sum_{j \in S} d_{j}=d i
$$

Now consider the polynomial

$$
P(t)=\prod_{i=1}^{r}\left(t^{d_{i} / d}+z_{i}\right) .
$$

For $1 \leqslant i \leqslant r$, the coefficient of $t^{r-i}$ in $P(t)$ is $\theta_{i, s}$. It follows that the elements $\left(-z_{i}\right)^{d / d_{i}}$ are integral over $W(k)\left[\theta_{1, s}, \ldots, \theta_{m, s}\right]$, and so the elements $z_{i}$ themselves are. As $W(k)\left[Z_{s}\right]$ is generated by the $z_{i}$, together with $\theta_{m, s}^{-1}$, it follows that $W(k)\left[Z_{s}\right]$ is integral, and hence finitely generated as a module, over $W(k)\left[\theta_{1, s}, \ldots, \theta_{m, s}, \theta_{m, s}^{-1}\right]$, and the result is immediate.

We now show:

Proposition 10.6. The module c-Ind ${ }_{K}^{G} \tau_{L_{s}^{\prime}}$ is an admissible $E_{K, \tau}[G]$-module. Moreover, for any $P^{\prime}=M^{\prime} U^{\prime}$ in $G, r_{G}^{P^{\prime}} \mathrm{c}-\operatorname{Ind}_{K}^{G} \tau_{L^{\prime}}$ is admissible as a $E_{K, \tau}\left[M^{\prime}\right]$ module.

Proof. The module $r_{G}^{P^{\prime}} \mathrm{c}-\operatorname{Ind}_{K}^{G} \tau_{L_{s}}$ is admissible over $R$, which we have identified with $\mathcal{O}^{\prime}\left[Z_{s}\right]^{W_{\bar{M}}^{(s)}}$. It is thus also admissible over $\mathcal{O}^{\prime}\left[\theta_{1, s}, \ldots, \theta_{m, s}, \theta_{m, s}^{-1}\right]$, by Proposition 10.5. The $\theta_{i, s}$ preserve both $\mathrm{c}-\operatorname{Ind}_{K}^{G} \tau_{L_{s}}$ and $\mathrm{c}-\operatorname{Ind}_{K}^{G} \tau_{L_{s}^{\prime}}$, and any embedding of the latter in the former is equivariant for the $\theta_{i, s}$ (as the action of these elements is via the Bernstein center). Fix such an embedding; this yields an embedding of $r_{G}^{P^{\prime}} \mathrm{c}-\operatorname{Ind}_{K}^{G} \tau_{L_{s}^{\prime}}$ in $r_{G}^{P^{\prime}} \mathrm{c}-\operatorname{Ind}_{K}^{G} \tau_{L_{s}}$ that is compatible with the action of the elements $\theta_{i, s}$. As the former is admissible over $\mathcal{O}^{\prime}\left[\theta_{1, s}, \ldots, \theta_{m, s}, \theta_{m, s}^{-1}\right]$, the latter must be as well, and the result follows.

We now return to the study of $\mathcal{P}_{K, \tau}$. We choose $\mathcal{K}^{\prime}$ (and by extension $\mathcal{O}^{\prime}$ ) sufficiently large that every map from $E_{\sigma}$ to $\overline{\mathcal{K}}$ has image contained in $\mathcal{O}^{\prime}$. Then 
$\mathrm{St}_{s}$ is defined over $\mathcal{K}^{\prime}$ for every $s$. Moreover, the embeddings:

$$
\begin{gathered}
\mathcal{P}_{K, \tau} \otimes \overline{\mathcal{K}} \hookrightarrow \bigoplus_{s} \mathrm{c}-\operatorname{Ind}_{K}^{G} \tilde{\kappa} \otimes \mathrm{St}_{s} \\
E_{K, \tau} \otimes \overline{\mathcal{K}} \hookrightarrow \prod_{s} H\left(G, K, \tilde{\kappa} \otimes \mathrm{St}_{s}\right)
\end{gathered}
$$

factor through embeddings:

$$
\begin{gathered}
\mathcal{P}_{K, \tau} \otimes \mathcal{O}^{\prime} \hookrightarrow \bigoplus_{s} \mathrm{c}-\operatorname{Ind}_{K}^{G} \tau_{L_{s}^{\prime}} \\
E_{K, \tau} \otimes \mathcal{O}^{\prime} \hookrightarrow \prod_{s} H\left(G, K, \tau_{L_{s}^{\prime}}\right)
\end{gathered}
$$

We thus have:

THEOREM 10.7. For any parabolic subgroup $P^{\prime}=M^{\prime} U^{\prime}$ of $G, r_{G}^{P^{\prime}} \mathcal{P}_{K, \tau}$ is an admissible $C_{K, \tau}\left[M^{\prime}\right]$-module (and hence also an admissible $E_{K, \tau}\left[M^{\prime}\right]$-module).

Proof. For each $s, r_{G}^{P^{\prime}} \mathrm{c}-\operatorname{Ind}_{K}^{G} \tau_{L_{s}^{\prime}}$ is admissible over $C_{K, \tau} \otimes \mathcal{O}^{\prime}$, and hence also over $C_{K, \tau}$. The result is thus immediate from the above decomposition.

COROLLARY 10.8. The $W(k)$-algebra $E_{K, \tau}$ is a finitely generated $C_{K, \tau}$-module.

We now turn to the study of the $W(k)[G]$-module $\mathcal{P}_{(M, \pi)}$ for an inertial equivalence class $(M, \pi)$ of irreducible cuspidal representations of $M$ over $k$. Write $\pi$ as a tensor product of irreducible cuspidal representations $\pi_{i}$ of general linear groups $\mathrm{GL}_{n_{i}}(F)$, and for each $i$, let $\left(K_{i}, \tau_{i}\right)$ be a maximal distinguished cuspidal $k$-type contained in $\pi_{i}$.

THEOREM 10.9. Let $R$ be the tensor product, over $W(k)$, of the rings $E_{K_{i}, \tau_{i}}$ for all $i$. Then $\mathcal{P}_{(M, \pi)}$ is an admissible $R[G]$-module, and for any parabolic subgroup $P^{\prime}=M^{\prime} U^{\prime}$ of $G, r_{G}^{P^{\prime}} \mathcal{P}_{(M, \pi)}$ is an admissible $R\left[M^{\prime}\right]$-module.

Proof. This is an immediate consequence of Theorem 10.7 and Lemma 10.3. $\square$

\section{The Bernstein decomposition and Bernstein's second adjointness}

Our next goal is to apply our results on the structure of $\mathcal{P}_{K, \tau}$ to establish a Bernstein decomposition for the category of smooth $W(k)[G]$-modules. With the results of the previous section in hand, this is an easy consequence of Bernstein's 
second adjointness for the category of smooth $W(k)[G]$-modules, which is due, in this generality, to Dat in [D3]. However, at this point it is not too much work to give an alternative proof of Bernstein's second adjointness which is quite different in spirit from Dat's approach. We thus detour for a moment to show how Bernstein's second adjointness follows from the results so far.

For technical reasons (namely, the fact that parabolic induction is naturally a right adjoint, and therefore takes injectives to injectives), it will be useful for us to work with injective objects rather than the projectives $\mathcal{P}_{K, \tau}$. To obtain a suitable supply of injectives, we define:

Definition 11.1. Let $\Pi$ be a smooth $W(k)[G]$-module. We denote by $\Pi^{\vee}$ the $W(k)[G]$-submodule of smooth vectors in $\operatorname{Hom}_{W(k)}(\Pi, \mathcal{K} / W(k))$.

As $\mathcal{K} / W(k)$ is an injective $W(k)$-module, and the functor that takes a $W(k)[G]$ module to the submodule consisting of its smooth vectors is exact, the functor $\Pi \mapsto \Pi^{\vee}$ is exact as well. Moreover, we have:

Lemma 11.2. Let $\Pi$ and $\Pi^{\prime}$ be smooth $W(k)[G]$-modules. Then there is a natural isomorphism:

$$
\operatorname{Hom}_{W(k)[G]}\left(\Pi,\left(\Pi^{\prime}\right)^{\vee}\right) \rightarrow \operatorname{Hom}_{W(k)[G]}\left(\Pi^{\prime}, \Pi^{\vee}\right) .
$$

Proof. Both $\operatorname{Hom}_{W(k)[G]}\left(\Pi,\left(\Pi^{\prime}\right)^{\vee}\right)$ and $\operatorname{Hom}_{W(k)[G]}\left(\Pi^{\prime}, \Pi^{\vee}\right)$ are in bijection with the set of $G$-equivariant pairings $\Pi \times \Pi^{\prime} \rightarrow \mathcal{K} / W(k)$ that are smooth with respect to the action of $G$.

COROLLARY 11.3. If $\Pi$ is a projective $W(k)[G]$-module, then $\Pi^{\vee}$ is injective.

Proof. The functors $\operatorname{Hom}_{W(k)[G]}\left(-, \Pi^{\vee}\right)$ and $\operatorname{Hom}_{W(k)[G]}\left(\Pi,(-)^{\vee}\right)$ are naturally equivalent, and the latter is exact.

This duality is well behaved with respect to normalized parabolic induction:

LEMMA 11.4. Let $P=M U$ be a Levi subgroup of $G$, and let $\pi$ be a smooth $W(k)[M]$-module. Then there is a natural isomorphism:

$$
\left(i_{P}^{G} \pi\right)^{\vee} \rightarrow i_{P}^{G} \pi^{\vee} .
$$

In particular, if $\Pi$ is a simple $W(k)[G]$-module with cuspidal (respectively supercuspidal) support $(M, \pi)$, then $\Pi^{\vee}$ has cuspidal (respectively supercuspidal) support $\left(M, \pi^{\vee}\right)$. 
Proof. Integration over $G / P$ defines a $G$-equivariant bilinear map:

$$
\left[i_{P}^{G} \pi\right] \times\left[i_{P}^{G} \pi^{\vee}\right] \rightarrow \mathcal{K} / W(k),
$$

and hence a $G$-equivariant map:

$$
i_{P}^{G} \pi^{\vee} \rightarrow\left[i_{p}^{G} \pi\right]^{\vee} .
$$

This map is easily seen to be an isomorphism by passing to $U$-invariants for a cofinal family of sufficiently small compact open subgroups $U$ of $G$.

Note that if $\Pi$ is an admissible $W(k)[G]$-module, (or, alternatively, an admissible $\mathcal{K}[G]$-module), then $\left(\Pi^{\vee}\right)^{\vee}$ is naturally isomorphic to $\Pi$. In particular, this is true if $\Pi$ is simple.

If $(K, \tau)$ is a maximal distinguished cuspidal $k$-type, we define $I_{K, \tau}$ to be the injective $W(k)[G]$-module $\mathcal{P}_{K, \tau^{\vee}}^{\vee}$. Similarly, if $(M, \pi)$ is a pair consisting of a Levi subgroup of $G$ and an irreducible cuspidal representation $\pi$ of $M$ over $k$, we set $I_{(M, \pi)}$ to be the $W(k)[G]$-module $\mathcal{P}_{\left(M, \pi^{\vee}\right)}^{\vee}$.

Proposition 11.5. The $W(k)[G]$-module $I_{(M, \pi)}$ is injective. Moreover, every simple $W(k)[G]$-module $\Pi$ with mod $\ell$ inertial cuspidal support $(M, \pi)$ embeds in $I_{(M, \pi)}$.

Proof. For a suitable parabolic subgroup $P$, and suitable maximal distinguished cuspidal types $\left(K_{i}, \tau_{i}\right)$, we have:

$$
\begin{aligned}
\mathcal{P}_{\left(M, \pi^{\vee}\right)}^{\vee} & =\left[i_{P}^{G} \mathcal{P}_{K_{1}, \tau_{1}^{\vee}} \otimes \cdots \otimes \mathcal{P}_{K_{r}, \tau_{r}^{\vee}}\right]^{\vee} \\
& =i_{P}^{G} I_{K_{1}, \tau_{1}} \otimes \cdots \otimes I_{K_{r}, \tau_{r}} .
\end{aligned}
$$

As $i_{P}^{G}$ is a right adjoint of an exact functor (by Frobenius reciprocity), the latter module is clearly injective.

Now given $\Pi, \Pi^{\vee}$ has mod $\ell$ inertial cuspidal support $\left(M, \pi^{\vee}\right)$; by Proposition 4.16 we have a surjection $\mathcal{P}_{\left(M, \pi^{\vee}\right)} \rightarrow \Pi^{\vee}$. Dualizing, and using the fact that $\left(\Pi^{\vee}\right)^{\vee}=\Pi$, we obtain our desired result.

Proposition 11.6. Let $(M, \pi)$ and $\left(M^{\prime}, \pi^{\prime}\right)$ be two pairs consisting of a Levi subgroup of $G$ and an irreducible cuspidal representation of that Levi subgroup over $k$. Let $I=I_{(M, \pi)} ; I^{\prime}=I_{\left(M^{\prime}, \pi^{\prime}\right)}$. If $\operatorname{Hom}_{W(k)[G]}\left(I, I^{\prime}\right)$ is nonzero, then the mod $\ell$ inertial supercuspidal supports of $(M, \pi)$ and $\left(M^{\prime}, \pi^{\prime}\right)$ coincide.

Proof. Set $\mathcal{P}=\mathcal{P}_{\left(M, \pi^{\vee}\right)} ; \mathcal{P}^{\prime}=\mathcal{P}_{\left(M^{\prime},\left(\pi^{\prime}\right)^{\vee}\right)}$, so that $I=\mathcal{P}^{\vee}$ and $I^{\prime}=\left(\mathcal{P}^{\prime}\right)^{\vee}$. We have an injection of $\operatorname{Hom}_{W(k)[G]}\left(I, I^{\prime}\right)$ into $\operatorname{Hom}_{W(k)[G]}\left(\left(I^{\prime}\right)^{\vee}, I^{\vee}\right)$; the latter is equal to 
$\operatorname{Hom}_{W(k)[G]}\left(\mathcal{P}^{\vee \vee},\left(\mathcal{P}^{\prime}\right)^{\vee \vee}\right)$. We also have an embedding:

$$
\operatorname{Hom}_{W(k)[G]}\left(\mathcal{P}^{\vee \vee},\left(\mathcal{P}^{\prime}\right)^{\vee \vee}\right) \rightarrow \operatorname{Hom}_{W(k)[G]}\left(\mathcal{P}^{\vee \vee} \otimes \overline{\mathcal{K}},\left(\mathcal{P}^{\prime}\right)^{\vee \vee} \otimes \overline{\mathcal{K}}\right),
$$

so it suffices to show that the latter is zero unless the $\bmod \ell$ inertial supercuspidal supports of $(M, \pi)$ and $\left(M^{\prime}, \pi^{\prime}\right)$ coincide.

We have an embedding of $\mathcal{P}^{\vee \vee} \otimes \overline{\mathcal{K}}$ into $(\mathcal{P} \otimes \overline{\mathcal{K}})^{\vee \vee}$.

If $\left(L, \pi^{\prime}\right)$ is a pair consisting of a Levi subgroup of $G$ and an irreducible supercuspidal representation of $L$ over $\overline{\mathcal{K}}$, and $\pi^{\prime}$ has mod $\ell$ inertial supercuspidal support different from $(M, \pi)$, then the projection $(\mathcal{P} \otimes \overline{\mathcal{K}})_{L, \pi}$ of $\mathcal{P} \otimes \overline{\mathcal{K}}$ to $\operatorname{Rep}_{\overline{\mathcal{K}}}(G)_{L, \pi}$ vanishes by part (2) of Theorem 9.8, and therefore so does $(\mathcal{P} \otimes \overline{\mathcal{K}})_{L, \pi}^{\vee}$. It follows that $(\mathcal{P} \otimes \overline{\mathcal{K}})^{\vee \vee}$ has a direct sum decomposition in which each summand lies in some block of $\operatorname{Rep}_{\overline{\mathcal{K}}}(G)$ corresponding to an inertial supercuspidal support whose $\bmod \ell$ reduction is $(M, \pi)$. Similarly, $\left(\mathcal{P}^{\prime} \otimes \overline{\mathcal{K}}\right)^{\vee \vee}$ has a direct summand decomposition in which each summand lies in some block of $\operatorname{Rep}_{\overline{\mathcal{K}}}(G)$ corresponding to an inertial supercuspidal support whose $\bmod \ell$ reduction is $\left(M^{\prime}, \pi^{\prime}\right)$.

Thus, if the $\bmod \ell$ inertial supercuspidal supports of $(M, \pi)$ and $\left.\left(M^{\prime}, \pi^{\prime}\right)\right\}$ differ, then no summand of $(\mathcal{P} \otimes \overline{\mathcal{K}})^{\vee \vee}$ lies in the same block as any summand of $\left(\mathcal{P}^{\prime} \otimes \overline{\mathcal{K}}\right)^{\vee \vee}$, and the result follows.

COROLlaRY 11.7. Every simple subquotient of $I_{(M, \pi)}$ has mod $\ell$ inertial supercuspidal support equal to that of $(M, \pi)$.

Proof. Let $\Pi$ be a simple subquotient of $I_{(M, \pi)}$, with $\bmod \ell$ inertial supercuspidal support $\left(M^{\prime}, \pi^{\prime}\right)$. Then $\Pi$ embeds in $I_{\left(M^{\prime}, \pi^{\prime}\right)}$; as the latter is injective we obtain a nonzero map $I_{(M, \pi)} \rightarrow I_{\left(M^{\prime}, \pi^{\prime}\right)}$. The preceding proposition now implies that $\left(M^{\prime}, \pi^{\prime}\right)$ has the same $\bmod \ell$ inertial supercuspidal support as $(M, \pi)$.

An immediate corollary is the 'Bernstein decomposition' for $\operatorname{Rep}_{W(k)}(G)$. Let $M$ be a Levi subgroup of $G$, and let $\pi$ be an irreducible supercuspidal representation of $M$ over $k$. If $\Pi$ is a simple smooth $W(k)[G]$-module with mod $\ell$ inertial supercuspidal support given by $(M, \pi)$, then the $\bmod \ell$ cuspidal support of $\Pi$ falls into one of finitely many possible $\bmod \ell$ inertial equivalence classes. Choose representatives $\left(M_{j}, \pi_{j}\right)$ for these inertial equivalence classes, and let $I_{[M, \pi]}=I_{\left(M_{1}, \pi_{1}\right)} \oplus \cdots \oplus I_{\left(M_{r}, \pi_{r}\right)}$. Then every simple subquotient of $I_{[M, \pi]}$ has mod $\ell$ inertial supercuspidal support $(M, \pi)$. On the other hand, any simple smooth $W(k)[G]$-module $\pi$ with $\bmod \ell$ inertial supercuspidal support $(M, \pi)$ has $\bmod \ell$ inertial cuspidal support $\left(M_{j}, \pi_{j}\right)$ for some $j$, and hence embeds in $I_{\left(M_{j}, \pi_{j}\right)}$ (and thus also in $\left.I_{[M, \pi]}\right)$. 
On the other hand, if $\Pi$ is a simple smooth $W(k)[G]$-module whose $\bmod$ $\ell$ inertial supercuspidal support is not in the inertial equivalence class $(M, \pi)$, then there is an infinite collection of possible inertial equivalence classes into which the $\bmod \ell$ inertial cuspidal support of $\Pi$ could fall. If we let $I_{\overline{[M, \pi]}}$ denote the direct sum of $I_{\left(M^{\prime}, \pi^{\prime}\right)}$ as $\left(M^{\prime}, \pi^{\prime}\right)$ runs over a set of representatives for the inertial equivalence classes of pairs $\left(M^{\prime}, \pi^{\prime}\right)$ over $k$ whose supercuspidal support is not in the inertial equivalence class $(M, \pi)$, then no subquotient of $I_{\overline{[M, \pi]}}$ has $\bmod \ell$ inertial supercuspidal support equal to $(M, \pi)$, and every simple object of $\operatorname{Rep}_{W(k)}(G)$ whose mod $\ell$ inertial supercuspidal support is not equivalent to $(M, \pi)$ is a subobject of $I_{\overline{[M, \pi]}}$.

THEOREM 11.8. The full subcategory $\operatorname{Rep}_{W(k)}(G)_{[M, \pi]}$ of $\operatorname{Rep}_{W(k)}(G)$ consisting of smooth $W(k)[G]$-modules $\Pi$ such that every simple subquotient of $\Pi$ has mod $\ell$ inertial supercuspidal support given by $(M, \pi)$ is a block of $\operatorname{Rep}_{W(k)}(G)$. Moreover, every element of $\operatorname{Rep}_{W(k)}(G)_{[M, \pi]}$ has a resolution by direct sums of copies of $I_{[M, \pi]}$.

Proof. This is immediate from the above discussion and Proposition 2.4.

Our first application of this Bernstein decomposition will be to establish Bernstein's second adjointness for smooth $W(k)[G]$-modules. This will allow us, at last, to conclude that the modules $\mathcal{P}_{(M, \pi)}$ are projective. We follow the argument in the lecture notes by Bernstein-Rumelhart [BR], adapting it as necessary so that it will work in $\operatorname{Rep}_{W(k)}(G)$.

Definition 11.9. Let $P=M U$ be a parabolic subgroup of $G$, let $K$ be a compact open subgroup of $G$ that is decomposed with respect to $P$, and let $\lambda$ be a totally positive central element of $M$. Let $T_{\lambda}$ be the element of $H(G, K, 1)$ given by $T^{+}\left(1_{K_{M} \lambda K_{M}}\right)$. A smooth $W(k)[G]$-module $\Pi$ is $K, P$-stable, with constant $c_{K, P, \lambda}$, if there exists a positive integer $c_{K, P, \lambda}$ such that $\Pi^{K}$ splits as a direct sum:

$$
\Pi^{K}=\Pi^{K}\left[T_{\lambda}^{c K, P, \lambda}\right] \oplus \Pi_{T_{\lambda} \text {-invert }}^{K},
$$

where $\Pi^{K}\left[T_{\lambda}^{c_{K, P, \lambda}}\right]$ is the $W(k)$-submodule of $\Pi^{K}$ consisting of elements killed by $T_{\lambda}^{c K, P, \lambda}$, and $\Pi_{T_{\lambda} \text {-invert }}^{K}$ is the maximal $W(k)$-submodule of $\Pi^{K}$ on which $[\lambda]$ is invertible.

The key to establishing Bernstein's second adjointness will be proving that for every pair $K, P$, and every supercuspidal inertial equivalence class $(L, \pi)$, all objects of $\operatorname{Rep}_{W(k)}(G)_{[L, \pi]}$ are $K, P$-stable. We first make a few observations: 
Lemma 11.10. Let $\Pi$ be a smooth $K, P$-stable $W(k)[G]$-module. Then $\Pi^{\vee}$ is also $K, P$-stable.

Proof. We have

$$
\left(\Pi^{\vee}\right)^{K} \cong\left(\Pi^{K}\right)^{\vee} \cong \Pi^{K}\left[T_{\lambda}^{c_{K, P, \lambda}}\right]^{\vee} \oplus\left[\Pi_{T_{\lambda}-\text { invert }}^{K}\right]^{\vee} ;
$$

the result follows immediately.

LEMMA 11.11. Finite direct sums of $K, P$-stable modules are $K, P$-stable. Infinite direct sums of modules which are $K, P$-stable with a uniform constant $c_{K, P, \lambda}$ are $K$-stable. Kernels and cokernels of maps of $K, P$-stable modules are $K, P$-stable.

LEMMA 11.12. Let $\Pi$ be a smooth $W(k)[G]$-module. Then the natural projection:

$$
\Pi^{K} \rightarrow\left(\Pi_{U}\right)^{K_{M}}
$$

identifies $\left(\Pi_{U}\right)^{K_{M}}$ with $\Pi^{K} \otimes_{W(k)\left[T_{\lambda}\right]} W(k)\left[T_{\lambda}, T_{\lambda}^{-1}\right]$. In particular, if $\Pi$ is $K, P$ stable, then the map $\Pi^{K} \rightarrow\left(\Pi_{U}\right)^{K_{M}}$ is surjective, and one has a direct sum decomposition:

$$
\Pi^{K}=\Pi^{K}\left[T_{\lambda}^{c_{K, P, \lambda}}\right] \oplus\left(\Pi_{U}\right)^{K_{M}} .
$$

This decomposition is independent of $\lambda$.

Proof. We make $\left(\Pi_{U}\right)^{K_{M}}$ into a $W(k)\left[T_{\lambda}\right]$-module by letting $T_{\lambda}$ act on $\left(\Pi_{U}\right)^{K_{M}}$ via $\lambda$. It is then clear that the map

$$
\Pi^{K} \rightarrow\left(\Pi_{U}\right)^{K_{M}}
$$

is $T_{\lambda}$-equivariant. It thus suffices to show that every element of the kernel of this map is killed by a power of $T_{\lambda}$, and that, for every element $x$ of $\left(\Pi_{U}\right)^{K_{M}}, \lambda^{m} x$ is in the image of this map for some sufficiently large $m$.

For the first claim, let $e_{K^{+}}$be the idempotent projector onto the $K^{+}$invariants of a $K$-module. As $K=K^{-} K_{M} K^{+}$, we have $e_{K}=e_{K^{+}} e_{K_{M}} e_{K^{-}}$. For each $m$, we have

$$
\begin{aligned}
e_{K} \lambda^{m} e_{K} & =e_{K^{+}} e_{K_{M}} e_{K^{-}} \lambda^{m} e_{K} \\
& =e_{K^{+}} \lambda^{m} e_{K_{M}} e_{\lambda^{-m} K^{-} \lambda^{m}} e_{K}=\lambda^{m} e_{\lambda^{-m} K^{+} \lambda^{m}} e_{K} .
\end{aligned}
$$

(Here we have used that $\lambda$ is positive.) Now if $\tilde{x}$ is an element of $\Pi^{K}$ that maps to zero in $\Pi_{U}$, then $e_{K} \tilde{x}=\tilde{x}$, and there exists a compact open subgroup $U_{1}$ of $U$ such that $e_{U_{1}} \tilde{x}=0$. But as $\lambda$ is strictly positive, there exists an $m$ such that 
$\lambda^{-m} K^{+} \lambda^{m}$ contains $U_{1}$. Thus $\tilde{x}$ is killed by $e_{\lambda^{-m} K^{+} \lambda^{m}}$ and fixed by $e_{K}$. Then $\tilde{x}$ is killed by $e_{K} \lambda^{m} e_{K}$, as required.

As for the second claim, let $\tilde{x}$ be a lift of $x$ to $\Pi^{K_{M}}$. There is a compact open subgroup $K^{\prime}$ of $G$ that fixes $\tilde{x}$; then $\tilde{x}$ is in particular invariant under $K^{\prime} \cap U^{\circ}$. As $\lambda$ is strictly positive, there exists an $m$ such that $\lambda^{m} \tilde{x}$ is invariant under $K^{-}$. Thus

$$
e_{K^{+}} \lambda^{m} \tilde{x}=e_{K^{+}} e_{K_{M}} e_{K^{-}} \lambda^{m} \tilde{x}=e_{K} \lambda^{m} \tilde{x},
$$

so $e_{K^{+}} \lambda^{m} \tilde{x}$ lies in $\Pi^{K}$. As $e_{K^{+}}$acts trivially on $\Pi_{U}$ (because $K^{+}$is contained in $U), e_{K^{+}} \lambda^{m} \tilde{x}$ maps to $\lambda^{m} \tilde{x}$ under the map

$$
\Pi^{K} \rightarrow\left(\Pi_{U}\right)^{K_{M}},
$$

as required.

Finally, if $\Pi$ is $K, P$-stable, then $\Pi^{K}$ surjects onto $\Pi^{K} \otimes_{W(k)\left[T_{\lambda}\right.} W(k)\left[T_{\lambda}, T_{\lambda}^{-1}\right]$; this surjection identifies $\left(\Pi_{U}\right)^{K_{M}}$ with the maximal $T_{\lambda}$-divisible submodule of $\Pi^{K}$ and thus yields the asserted direct sum decomposition. To see that this decomposition is independent of $\lambda$, choose another strictly positive element $\lambda^{\prime}$. Then the maximal $T_{\lambda \lambda^{\prime}}$-divisible submodule of $\Pi^{K}$ is contained in both the maximal $T_{\lambda}$-divisible submodule and the maximal $T_{\lambda^{\prime}}$-divisible submodule; since projection onto $\left(\Pi_{U}\right)^{K_{M}}$ is an isomorphism on each of these submodules they must all coincide.

LEMMA 11.13. Let $R$ be a commutative Noetherian $W(k)$-algebra, and let $\Pi$ be an admissible $R[G]$-module. Suppose that $r_{G}^{P} \Pi$ is also admissible. Then $\Pi$ is K, P-stable.

Proof. As $r_{G}^{P} \Pi$ is a twist of $\Pi_{U}$, the hypotheses imply that $\Pi^{K}$ and $\left(\Pi_{U}\right)^{K_{M}}$ are finitely generated $R$-modules. On the other hand, we have an isomorphism

$$
\left(\Pi_{U}\right)^{K_{M}} \cong \Pi^{K} \otimes_{R\left[T_{\lambda}\right]} R\left[T_{\lambda}, T_{\lambda}^{-1}\right]
$$

The result is now an immediate consequence of [BR, Lemma 33].

COROLLARY 11.14. For any Levi subgroup $L$ of $G$, and any irreducible cuspidal representation $\pi$ of $L$ over $k$, the modules $\mathcal{P}_{(M, \pi)}$ and $I_{(M, \pi)}$ are $K, P$-stable.

Proof. For $\mathcal{P}_{(M, \pi)}$, the result follows from the previous lemma, together with Theorem 10.9. We also have the result for $\mathcal{P}_{[M, \pi]}$, as this is a finite direct sum of modules $\mathcal{P}_{\left[M^{\prime}, \pi^{\prime}\right]}$. As $I_{(M, \pi)}=\mathcal{P}_{\left(M, \pi^{\vee}\right)}^{\vee}$, we also have the result for $I_{(M, \pi)}$, and hence also for $I_{[M, \pi]}$. 
Proposition 11.15. For any Levi subgroup $L$ of $G$, and any irreducible supercuspidal k-representation $\pi$ of $L$, every object of $\operatorname{Rep}_{W(k)}(G)_{[L, \pi]}$ is $K, P$ stable.

Proof. Any object of $\operatorname{Rep}_{W(k)}(G)_{[L, \pi]}$ has a resolution by direct sums of $I_{[L, \pi]}$. These are $K, P$-stable, so the result follows from the fact that kernels of maps of $K, P$-stable modules are $K, P$-stable.

It follows that for any object $\Pi$ of $\operatorname{Rep}_{W(k)}(G)_{[L, \pi]}$, the map $\Pi^{K} \rightarrow\left(\Pi_{U}\right)^{K_{M}}$ is surjective.

PROPOSITION 11.16. Let $\Pi$ be an object of $\operatorname{Rep}_{W(k)}(G)_{[L, \pi]}$. There is a canonical isomorphism:

$$
r_{G}^{P} \Pi^{\vee} \cong\left(r_{G}^{P^{\circ}} \Pi\right)^{\vee}
$$

Proof. We follow the proof of [B, Theorem 2]. First note that $r_{G}^{P} \Pi^{\vee}$ is a twist of $\left(\Pi^{\vee}\right)_{U}$ by our (fixed) square root of the modulus character of $P$, and $r_{G}^{P^{\circ}} \Pi$ is a twist of $\Pi_{U^{\circ}}$ by a square root of the modulus character of $P^{\circ}$. As these two modulus characters are inverses of each other, it suffices to construct an isomorphism $\left(\Pi_{U^{\circ}}\right)^{\vee} \cong\left(\Pi^{\vee}\right)_{U}$ of $W(k)[M]$-modules.

For each $K$ that is decomposed with respect to $P$, we have an isomorphism:

$$
\left(\Pi^{K}\right)^{\vee} \rightarrow\left(\Pi^{\vee}\right)^{K}
$$

coming from a perfect pairing $\Pi^{K} \times\left(\Pi^{\vee}\right)^{K} \rightarrow \mathcal{K} / W(k)$. Under this pairing, the adjoint of $T_{\lambda}$ is $T_{\lambda^{-1}}$. If we take $\lambda$ to be strictly positive with respect to $P$, then $\lambda^{-1}$ is strictly positive with respect to $P^{\circ}$. Moreover, $\Pi$ is both $(K, P)$ stable and $\left(K, P^{\circ}\right)$-stable. In particular, $\left(\Pi_{U^{\circ}}\right)^{K_{M}}$ is isomorphic to the maximal $T_{\lambda^{-1}}$-divisible submodule of $\Pi^{K}$, and $\left(\left(\Pi^{\vee}\right)_{U}\right)^{K_{M}}$ is isomorphic to the maximal $T_{\lambda}$-divisible submodule of $\left(\Pi^{\vee}\right)^{K}$. Moreover, under these identifications $\left(\Pi_{U^{\circ}}\right)^{K_{M}}$ and $\left(\left(\Pi^{\vee}\right)_{U}\right)^{K_{M}}$ are direct summands of $\Pi^{K}$ and $\left(\Pi^{\vee}\right)^{K}$, respectively. The pairing on the latter thus descends to a perfect pairing:

$$
\left(\Pi_{U^{\circ}}\right)^{K_{M}} \times\left(\left(\Pi^{\vee}\right)_{U}\right)^{K_{M}} \rightarrow \mathcal{K} / W(k) .
$$

By [B], Lemma 1, we can find a $K$ decomposed with respect to $P$ inside any compact open subgroup of $G$. Taking the limit over a cofinal system of such $K$ gives the desired perfect pairing

$$
\Pi_{U^{\circ}} \times\left(\Pi^{\vee}\right)_{U} \rightarrow \mathcal{K} / W(k)
$$

and hence the desired identification of $\left(\Pi^{\vee}\right)_{U}$ with $\left(\Pi_{U^{\circ}}\right)^{\vee}$. 
THEOREM 11.17 (Bernstein's second adjointness). Let $\Pi_{1}$ and $\Pi_{2}$ be objects of the categories $\operatorname{Rep}_{W(k)}(M)$ and $\operatorname{Rep}_{W(k)}(G)_{[L, \pi]}$, respectively. Then there is a canonical isomorphism:

$$
\operatorname{Hom}_{W(k)[G]}\left(i_{P}^{G} \Pi_{1}, \Pi_{2}\right) \cong \operatorname{Hom}_{W(k)[M]}\left(\Pi_{1}, r_{G}^{P^{\circ}} \Pi_{2}\right) .
$$

Proof. We follow the argument of the 'claim' after [BR, Theorem 20]. We first establish the case in which $\Pi_{2}=\left(\Pi_{2}^{\prime}\right)^{\vee}$ for some $\Pi_{2}^{\prime}$ in $\operatorname{Rep}_{W(k)}(G)_{\left[L, \pi^{\vee}\right]}$. We then have a sequence of functorial isomorphisms:

$$
\begin{aligned}
\operatorname{Hom}_{W(k)[M]}\left(\Pi_{1}, r_{G}^{P^{\circ}} \Pi_{2}\right) & \cong \operatorname{Hom}_{W(k)[M]}\left(\Pi_{1},\left(r_{G}^{P} \Pi_{2}^{\prime}\right)^{\vee}\right) \\
& \cong \operatorname{Hom}_{W(k)[M]}\left(r_{G}^{P} \Pi_{2}^{\prime}, \Pi_{1}^{\vee}\right) \\
& \cong \operatorname{Hom}_{W(k)[G]}\left(\Pi_{2}^{\prime}, i_{P}^{G} \Pi_{1}^{\vee}\right) \\
& \cong \operatorname{Hom}_{W(k)[G]}\left(\Pi_{2}^{\prime},\left(i_{P}^{G} \Pi_{1}\right)^{\vee}\right) \\
& \cong \operatorname{Hom}_{W(k)[G]}\left(i_{P}^{G} \Pi_{1}, \Pi_{2}\right)
\end{aligned}
$$

In particular, the result holds for $\Pi_{2}=I_{[L, \pi]}$. If $\Pi_{1}$ is finitely generated, then the functors $\operatorname{Hom}_{W(k)[G]}\left(i_{P}^{G} \Pi_{1},-\right)$ and $\operatorname{Hom}_{W(k)[M]}\left(\Pi_{1}, r_{G}^{P^{\circ}-}\right)$ commute with arbitrary direct sums, so the result holds for $\Pi_{1}$ finitely generated and $\Pi_{2}$ an arbitrary direct sum of copies of $I_{[L, \pi]}$. As any $\Pi_{1}$ is the limit of its finitely generated submodules, the result holds for an arbitrary $\Pi_{1}$, when $\Pi_{2}$ is an arbitrary direct sum of copies of $I_{[L, \pi]}$. Finally, we can resolve an arbitrary $\Pi_{2}$ by direct sums of copies of $I_{[L, \pi]}$, and the result then follows for all $\Pi_{1}, \Pi_{2}$.

COROLLARY 11.18. The representations $\mathcal{P}_{[L, \pi]}$ are projective and small.

Proof. It suffices to show that each $\mathcal{P}_{\left(M, \pi^{\prime}\right)}$, with $\pi^{\prime}$ irreducible and cuspidal over $K$ is projective and small, as $\mathcal{P}_{[L, \pi]}$ is a finite direct sum of representations of this form. For a suitable sequence of types $\left(K_{i}, \tau_{i}\right)$, we have

$$
\mathcal{P}_{\left(M, \pi^{\prime}\right)}=i_{P}^{G} \mathcal{P}_{K_{1}, \tau_{1}} \otimes \cdots \otimes \mathcal{P}_{K_{r}, \tau_{r}} .
$$

For each $i$ the representation $\mathcal{P}_{K_{i}, \tau_{i}}$ is projective and small (as $\mathcal{P}_{K_{i}, \tau_{i}}$ is the compact induction of a finite-length $W(k)$-module). Thus the tensor product $\Pi$ of the $\mathcal{P}_{K_{i}, \tau_{i}}$ is projective and small when considered as a $W(k)[M]$-module. We have shown that $\mathcal{P}_{\left(M, \pi^{\prime}\right)}$ lies in $\operatorname{Rep}_{W(k)}(G)_{[L, \pi]}$.

Fix a smooth representation $\Pi^{\prime}$ of $G$, and let $\Pi_{[L, \pi]}^{\prime}$ be the direct summand of $\Pi^{\prime}$ that lies in $\operatorname{Rep}_{W(k)}(G)_{[L, \pi]}$. Then

$$
\begin{aligned}
\operatorname{Hom}_{W(k)[G]}\left(\mathcal{P}_{\left(M, \pi^{\prime}\right)}, \Pi^{\prime}\right) & \cong \operatorname{Hom}_{W(k)[G]}\left(\mathcal{P}_{\left(M, \pi^{\prime}\right)}, \Pi_{[L, \pi]}^{\prime}\right) \\
& \cong \operatorname{Hom}_{W(k)[M]}\left(\Pi, r_{G}^{P^{\circ}} \Pi_{[L, \pi]}^{\prime}\right)
\end{aligned}
$$


As $r_{G}^{P^{\circ}}$ commutes with direct sums it is easy to see this implies $\mathcal{P}_{\left(M, \pi^{\prime}\right)}$ is small; projectivity of $\mathcal{P}_{\left(M, \pi^{\prime}\right)}$ follows from exactness of $r_{G}^{P^{\circ}}$.

COROLlaRy 11.19. The Bernstein center $A_{[L, \pi]}$ of $\operatorname{Rep}_{W(k)}(G)_{[L, \pi]}$ is isomorphic to the center of $\operatorname{End}_{W(k)[G]}\left(\mathcal{P}_{[L, \pi]}\right)$.

Proof. Every simple object of $\operatorname{Rep}_{W(k)}(G)_{[L, \pi]}$ is a quotient of $\mathcal{P}_{\left(M, \pi^{\prime}\right)}$ for some pair $\left(M, \pi^{\prime}\right)$ with inertial supercuspidal support $(L, \pi)$, and hence such an object is a quotient of $\mathcal{P}_{[L, \pi]}$. It follows that $\mathcal{P}_{[L, \pi]}$ is faithfully projective in $\operatorname{Rep}_{W(k)}(G)_{[L, \pi]}$, and the result follows immediately.

\section{Structure of the Bernstein center}

In this section we prove basic facts about the center $A_{[L, \pi]}$ of $\operatorname{End}_{W(k)[G]}\left(\mathcal{P}_{[L, \pi]}\right)$.

PROPOSITION 12.1. There is a natural isomorphism:

$$
A_{[L, \pi]} \otimes \overline{\mathcal{K}} \cong \prod_{(\tilde{M}, \tilde{\pi})} A_{\tilde{M}, \tilde{\pi}},
$$

where $(\tilde{M}, \tilde{\pi})$ runs over inertial equivalence classes of pairs in which $\tilde{M}$ is a Levi subgroup of $G$ and $\tilde{\pi}$ is a cuspidal representation of $M$ over $\overline{\mathcal{K}}$ whose mod $\ell$ inertial supercuspidal support equals $(L, \pi)$. This isomorphism is uniquely characterized by the property that for any $\Pi$ in $\operatorname{Rep}_{\overline{\mathcal{K}}}(G)$, and any $x$ in $A_{[L, \pi]}$, the action of $x$ on $\Pi$ coincides with that of its image in $\prod_{(\tilde{M}, \tilde{\pi})} A_{\tilde{M}, \tilde{\pi}}$.

Proof. The module $\mathcal{P}$ defined by $\mathcal{P}=\mathrm{c}-\operatorname{Ind}_{\{e\}}^{G} W(k)$, where $\{e\}$ is the trivial subgroup of $G$, is a faithfully projective module in $\operatorname{Rep}_{W(k)}(G)$. We have

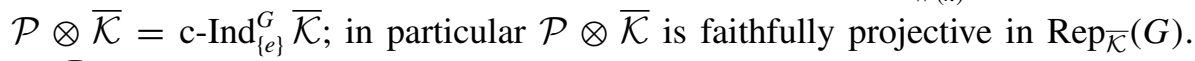
As $\mathcal{P}$ is $\ell$-torsion free, we have an injection:

$$
\operatorname{End}_{W(k)[G]}(\mathcal{P}) \rightarrow \operatorname{End}_{W(k)[G]}(\mathcal{P}) \otimes \overline{\mathcal{K}} \cong \operatorname{End}_{\overline{\mathcal{K}}[G]}(\mathcal{P} \otimes \overline{\mathcal{K}})
$$

In particular, we have an isomorphism:

$$
Z\left(\operatorname{End}_{W(k)[G]}(\mathcal{P})\right) \otimes \overline{\mathcal{K}} \cong Z\left(\operatorname{End}_{\overline{\mathcal{K}}[G]}(\mathcal{P} \otimes \overline{\mathcal{K}})\right)
$$

Multiplying both sides by the central idempotent $e_{[L, \pi]}$ gives us the desired isomorphism. This isomorphism is equivariant for the actions of both sides on $\mathcal{P} \otimes \overline{\mathcal{K}}$, and hence for all objects of $\operatorname{Rep}_{\overline{\mathcal{K}}}(G)$. 
As $\mathcal{P}_{[L, \pi]}$ is, by definition, a faithful $A_{[L, \pi]}$-module, we have injections:

$$
\begin{gathered}
A_{[L, \pi]} \otimes \overline{\mathcal{K}} \hookrightarrow \operatorname{End}_{\overline{\mathcal{K}}[G]}\left(\mathcal{P}_{[L, \pi]} \otimes \overline{\mathcal{K}}\right) \\
\operatorname{End}_{W(k)[G]}\left(\mathcal{P}_{[L, \pi]}\right) \hookrightarrow \operatorname{End}_{\overline{\mathcal{K}}[G]}\left(\mathcal{P}_{[L, \pi]} \otimes \overline{\mathcal{K}}\right),
\end{gathered}
$$

and it is clear that $A_{[L, \pi]}$ is the intersection, inside $\operatorname{End}_{\overline{\mathcal{K}}[G]}\left(\mathcal{P}_{[L, \pi]} \otimes \overline{\mathcal{K}}\right)$, of these two subalgebras. Thus we can identify $A_{[L, \pi]}$ with the set of elements $x$ in $\prod_{(\tilde{M}, \tilde{\pi})} A_{\tilde{M}, \tilde{\pi}}$ such that the action of $x$ on $\mathcal{P}_{[L, \pi]} \otimes \overline{\mathcal{K}}$ preserves $\mathcal{P}_{[L, \pi]}$.

We now study the interaction between $A_{[L, \pi]}$ and parabolic induction. We first show:

LEMMA 12.2. Let $P$ be a parabolic subgroup of $G$, with Levi subgroup $M$, and let $\Pi$ be a smooth $W(k)[M]$-module that is $\ell$-torsion free. Then the natural map:

$$
\operatorname{End}_{W(k)[M]}(\Pi) \rightarrow \operatorname{End}_{W(k)[M]}\left(i_{P}^{G} \Pi\right)
$$

is injective. Moreover, if $f$ is an element of $\operatorname{End}_{W(k)[G]}\left(i_{P}^{G} \Pi\right)$ such that $\ell^{a} f$ is in the image of this map for some a, then $f$ is also in the image of this map.

Proof. Let $g$ be an element of $\operatorname{End}_{W(k)[M]}(\Pi)$. Then $g$ fits in a commutative diagram:

$$
\begin{array}{cc}
r_{G}^{P} i_{P}^{G} \Pi & \rightarrow \Pi \\
\downarrow & \downarrow \\
r_{G}^{P} i_{P}^{G} \Pi & \rightarrow \Pi
\end{array}
$$

in which the horizontal arrows are the natural maps (and are therefore surjective), and the vertical arrows are $r_{G}^{P} i_{P}^{G} g$ and $g$, respectively. The surjectivity of the horizontal maps shows we can recover $g$ from $r_{G}^{P} i_{P}^{G} g$, proving the first claim. As for the second, suppose that $\ell^{a} f=i_{P}^{G} g$ for some $g$. Then the image of $r_{G}^{P} i_{P}^{G} g$ is contained in $\ell^{a} r_{G}^{P} i_{P}^{G} \Pi$ and the above diagram shows that the image of $g$ is contained in $\ell^{a} \Pi$. As $\Pi$ is $\ell$-torsion free it follows that $g$ is (uniquely) divisible by $\ell^{a}$ in $\operatorname{End}_{W(k)[M]}(\Pi)$; that is, $g=\ell^{a} g^{\prime}$. Then we have $f=i_{P}^{G} g^{\prime}$.

Now let $L^{\prime}$ be a Levi subgroup of $G$ containing $L$, such that $L^{\prime}$ is a product of factors $L_{i}^{\prime}$ isomorphic to $\mathrm{GL}_{n_{i}}(F)$. Then if $L_{i}$ is the Levi subgroup $L \cap L_{i}^{\prime}$ of $L_{i}^{\prime}$, we have $L=\prod_{i} L_{i}$ and $\pi$ factors as a tensor product of supercuspidal representations $\pi_{i}$ of $L_{i}$. In this situation we have:

THEOREM 12.3. Suppose $V$ lies in $\operatorname{Rep}_{W(k)}(M)_{[L, \pi]}$, and let $P$ be a parabolic subgroup of $G$ with Levi component $M$. Then $i_{P}^{G} V$ lies in $\operatorname{Rep}_{W(k)}(G)_{[L, \pi]}$. Moreover, there is a unique map: $f: A_{[L, \pi]} \rightarrow \bigotimes_{i} A_{\left[L_{i}, \pi_{i}\right]}$ such that, for all $V$ 
in $\operatorname{Rep}_{W(k)}(M)_{[L, \pi]}$, the diagram:

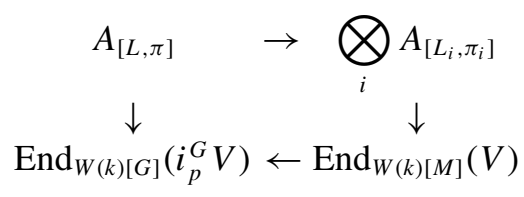

commutes. Moreover, $f$ is independent of $P$.

Proof. Let $V$ bet the faithfully projective $W(k)[M]$-module $\bigotimes_{i} \mathcal{P}_{\left[L_{i}, \pi_{i}\right]}$. Then $V$ is a direct sum of representations of the form $\bigotimes_{i} \mathcal{P}_{M_{i}^{\prime}, \pi_{i}^{\prime}}$, where for each $i, M_{i}^{\prime}$ is a Levi subgroup of $M_{i}$ and $\pi_{i}^{\prime}$ is an irreducible cuspidal representation of $M_{i}^{\prime}$ over $k$ with inertial supercuspidal support $\left(L_{i}, \pi_{i}\right)$.

Fix a collection $\left(M_{i}^{\prime}, \pi_{i}^{\prime}\right)$, let $M^{\prime}$ be the product of the $M_{i}^{\prime}$ and let $\pi^{\prime}$ be the tensor product of the $\pi_{i}^{\prime}$. Then $i_{P}^{G} \bigotimes_{i} \mathcal{P}_{M_{i}^{\prime}, \pi_{i}^{\prime}}$ is isomorphic to projective representation $\mathcal{P}_{M^{\prime}, \pi^{\prime}}$, and $\left(M^{\prime}, \pi^{\prime}\right)$ has inertial supercuspidal support $(L, \pi)$. In particular, $i_{P}^{G} \bigotimes_{i} \mathcal{P}_{M_{i}^{\prime}, \pi_{i}^{\prime}}$ lies in $\operatorname{Rep}_{W(k)}(G)_{[L, \pi]}$. Since $i_{P}^{G} V$ is a direct sum of these projectives, it too lies in $\operatorname{Rep}_{W(k)}(G)_{[L, \pi]}$.

On the other hand, any object of $\operatorname{Rep}_{W(k)}(M)_{[L, \pi]}$ has a resolution by direct sums of copies of $V$, so its parabolic induction has a resolution by direct sums of copies of $i_{P}^{G} V$ and hence lies in $\operatorname{Rep}_{W(k)}(G)_{[L, \pi]}$.

We now turn to the second claim. We first construct the map $f$ over $\overline{\mathcal{K}}$. In this case we have product decompositions:

$$
\begin{aligned}
& A_{[L, \pi]} \otimes \overline{\mathcal{K}} \cong \prod A_{M, \tilde{\pi}} \\
& (\tilde{M}, \tilde{\pi}) \\
& \bigotimes_{i} A_{\left[L_{i}, \pi_{i}\right]} \otimes \overline{\mathcal{K}} \cong \bigotimes_{i} \prod_{\left(\tilde{M}_{i}, \tilde{\pi}_{i}\right)} A_{\tilde{M}_{i}, \tilde{\pi}_{i}},
\end{aligned}
$$

where $\tilde{M}, \tilde{\pi}$ runs over pairs with $\bmod \ell$ inertial supercuspidal support $[L, \pi]$ and $\tilde{M}_{i}, \tilde{\pi}_{i}$ runs over pairs with $\bmod \ell$ inertial supercuspidal support $\left[L_{i}, \pi_{i}\right]$.

Define $f \otimes \overline{\mathcal{K}}$ on $A_{\tilde{M}, \tilde{\pi}} \rightarrow \bigotimes_{i} A_{\tilde{M}_{i}, \tilde{\pi}_{i}}$ to be the map $\Phi$ described immediately before Proposition 3.6 if $\left(\prod_{i} \tilde{M}_{i}, \otimes_{i} \tilde{\pi}_{i}\right)$ is inertially equivalent to $(M, \tilde{\pi})$, and the zero map otherwise. It is clear from Proposition 3.6 that the resulting $f \otimes \overline{\mathcal{K}}$ has the desired property with respect to parabolic induction.

Since $W(k)$ contains a square root of $q$, parabolic induction is compatible with twisting by elements of $\operatorname{Gal}(\overline{\mathcal{K}} / K)$, and from this we see that $f \otimes \overline{\mathcal{K}}$ must be $\operatorname{Gal}(\overline{\mathcal{K}} / K)$-equivariant. In particular, $f \otimes \overline{\mathcal{K}}$ descends to a map $f \otimes \mathcal{K}$ from $A_{[L, \pi]} \otimes \mathcal{K}$ to $\bigotimes_{i} A_{\left[L_{i}, \pi_{i}\right]} \otimes \mathcal{K}$. This latter map also has the desired property with respect to parabolic induction. 
Now let $x$ be an element of $A_{[L, \pi]}$, and let $y$ be the image of $x$ under $f \otimes \mathcal{K}$. Then $y$ lies in $\bigotimes_{i} A_{\left[L_{i}, \pi_{i}\right]} \otimes \mathcal{K}$, and so there exists a power $\ell^{a}$ of $\ell$ such that $\ell^{a} y$ lies in $\bigotimes_{i} A_{\left[L_{i}, \pi_{i}\right]}$. Now $x$ acts on $i_{P}^{G} V$, and the action of $\ell^{a} x$ on $i_{P}^{G} V$ coincides with that of $\ell^{a} y$. It follows by Lemma 12.2 that there is an element $y^{\prime}$ of $\operatorname{End}_{W(k)[M]}(V)$ such that the action of $x$ on $i_{P}^{G}$ coincides with that of $y^{\prime}$. Since $\ell^{a} y$ and $\ell^{a} y^{\prime}$ agree on $i_{P}^{G} V$, and $V$ is $\ell$-torsion free, we must have $y^{\prime}=y$. Thus $y$ lies in the intersection of $\bigotimes_{i} A_{\left[L_{i}, \pi_{i}\right]} \otimes \mathcal{K}$ with $\operatorname{End}_{W(k)[M]}(V)$, and we have seen that this intersection is precisely $\bigotimes_{i} A_{\left[L_{i}, \pi_{i}\right]}$.

We have thus constructed the desired $f$, and shown that the given diagram commutes for our particular choice of $V$. But every object of $\operatorname{Rep}_{W(k)}(M)_{[L, \pi]}$ has a resolution by direct sums of copies of $V$, so the diagram commutes for an arbitrary object of this category.

Finally, since $f \otimes \overline{\mathcal{K}}$ does not depend on $P$, neither does $f$.

In one particularly important case the map arising from parabolic restriction is an isomorphism. Call a pair $(L, \pi)$ simple if $L$ can be written as a product of copies of $\mathrm{GL}_{m}(F)$ for some fixed $m$, and $\pi$ is inertially equivalent to a tensor product $\left(\pi^{\prime}\right)^{n / m}$ for some fixed supercuspidal representation $\pi^{\prime}$ of $\operatorname{GL}_{m}(F)$. Say two simple types $\left(L_{1}, \pi_{1}\right)$ and $\left(L_{2}, \pi_{2}\right)$ overlap if there exist positive integers $m, j_{1}, j_{2}$, and a supercuspidal representation $\pi^{\prime}$ of $\mathrm{GL}_{m}(F)$, such that $L_{1}$ and $L_{2}$ are isomorphic to $\mathrm{GL}_{m}(F)^{j_{1}}$ and $\mathrm{GL}_{m}(F)^{j_{2}}$, respectively, $\pi_{1}$ and $\pi_{2}$ are inertially equivalent to $\left(\pi^{\prime}\right)^{\otimes j_{1}}$ and $\left(\pi^{\prime}\right)^{\otimes j_{2}}$, respectively. If $\left(L_{1}, \pi_{2}\right)$ and $\left(L_{2}, \pi_{2}\right)$ do not overlap, we say they are disjoint.

Given an arbitrary pair $(L, \pi)$, there is, up to inertial equivalence, a unique collection $\left\{\left(L_{i}, \pi_{i}\right)\right\}$ of disjoint simple pairs such that $L$ is the product of the $L_{i}$, and $\pi$ is the tensor product of the $\pi_{i}$. We call this collection the canonical factorization of $(L, \pi)$ into simple types.

Given such a factorization, there is a unique Levi subgroup $M$ of $G$ containing $L$ such that $M$ is a product of general linear groups $M_{i}$ with $M_{i} \cap L=L_{i}$ for all $i$. We denote by $\operatorname{Rep}_{W(k)}(M)_{\left\{\left[L_{i}, \pi_{i}\right]\right\}}$ the block of $\operatorname{Rep}_{W(k)}(M)$ whose simple objects $V$ have the form $\otimes_{i} V_{i}$ with $V_{i}$ a simple object of $\operatorname{Rep}_{W(k)}\left(M_{i}\right)_{\left[L_{i}, \pi_{i}\right]}$ for all $i$. The center $A_{\left\{\left[L_{i}, \pi_{i}\right]\right\}}$ of $\operatorname{Rep}_{W(k)}(M)_{\left\{\left[L_{i}, \pi_{i}\right]\right\}}$ is isomorphic to $\bigotimes_{i} A_{\left[L_{i}, \pi_{i}\right]}$. Let $P$ be a parabolic subgroup of $G$ with Levi $M$. Then parabolic induction $i_{P}^{G}$ takes $\operatorname{Rep}_{W(k)}(M)_{\left\{\left[L_{i}, \pi_{i}\right]\right\}}$ to $\operatorname{Rep}_{W(k)}(G)_{[L, \pi]}$.

THEOREM 12.4. Let $\left\{\left(L_{i}, \pi_{i}\right)\right\}$ be the factorization of $(L, \pi)$ into disjoint simple pairs. Then the functor

$$
i_{P}^{G}: \operatorname{Rep}_{W(k)}(M)_{\left\{\left[L_{i}, \pi_{i}\right]\right\}} \rightarrow \operatorname{Rep}_{W(k)}(G)_{[L, \pi]}
$$


is an equivalence of categories. In particular, the map map:

$$
f: A_{[L, \pi]} \rightarrow \bigotimes_{i} A_{\left[L_{i}, \pi_{i}\right]}
$$

arising from Theorem 12.3 is an isomorphism.

Proof. Let $\left(M^{\prime}, \pi^{\prime}\right)$ be a pair, with $M^{\prime}$ a Levi containing $L$ and $\pi^{\prime}$ a cuspidal representation of $M^{\prime}$ with inertial supercuspidal support $(L, \pi)$. Then there is a unique collection $\left\{\left(M_{i}^{\prime}, \pi_{i}^{\prime}\right)\right\}$ such that $M^{\prime}$ is the product of the $M_{i}^{\prime}, \pi^{\prime}$ is the tensor product of the $\pi_{i}^{\prime}$, and each $\pi_{i}^{\prime}$ has inertial supercuspidal support $\left(L_{i}, \pi_{i}\right)$. From this it follows that $\mathcal{P}_{M^{\prime}, \pi^{\prime}}$ is isomorphic to $i_{P}^{G} \bigotimes_{i} \mathcal{P}_{M_{i}^{\prime}, \pi_{i}^{\prime}}$. Taking direct sums, we find that $\mathcal{P}_{[L, \pi]}$ is isomorphic to $i_{P}^{G} \bigotimes_{i} \mathcal{P}_{\left[L_{i}, \pi_{i}\right]}$.

Let $e_{\left\{\left[L_{i}, \pi_{i}\right]\right\}}$ be the idempotent corresponding to the block $\operatorname{Rep}_{W(k)}(M)_{\left\{\left[L_{i}, \pi_{i}\right]\right\}}$ of $\operatorname{Rep}_{W(k)}(M)$. From Bernstein-Zelevinsky's filtration of the composite functor $r_{G}^{P} i{ }_{P}^{G}$, we see that the natural map

$$
r_{G}^{P} i_{P}^{G} \bigotimes_{i} \mathcal{P}_{\left[L_{i}, \pi_{i}\right]} \rightarrow \bigotimes_{i} \mathcal{P}_{\left[L_{i}, \pi_{i}\right]}
$$

is a surjection, whose kernel is annihilated by $e_{\left\{\left[L_{i}, \pi_{i}\right]\right\}}$. We thus have natural isomorphisms:

$$
\begin{aligned}
\bigotimes_{i} \mathcal{P}_{\left[L_{i}, \pi_{i}\right]} & \cong e_{\left\{\left[L_{i}, \pi_{i}\right]\right\}} r_{G}^{P} i_{P}^{G} \bigotimes_{i} \mathcal{P}_{\left[L_{i}, \pi_{i}\right]} \\
\mathcal{P}_{[L, \pi]} & \cong i_{P}^{G} e_{\left\{\left[L_{i}, \pi_{i}\right]\right\}} r_{G}^{P} \mathcal{P}_{[L, \pi]} .
\end{aligned}
$$

From this one deduces that the pair of functors $i_{P}^{G}$ and $e_{\left\{\left[L_{i}, \pi_{i}\right]\right\}} r_{G}^{P}$ are inverses. For instance, any object $V$ of $\operatorname{Rep}_{W(k)}(M)_{\left\{\left[L_{i}, \pi_{i}\right]\right\}}$ admits a resolution by direct sums of copies of $\bigotimes_{i} \mathcal{P}_{\left[L_{i}, \pi_{i}\right]}$. Applying the functor $e_{\left\{\left[L_{i}, \pi_{i}\right]\right\}} r_{G}^{P} i_{P}^{G}$ to this resolution yields a complex isomorphic to the original one via the natural isomorphism described above. The remainder of the argument is similar.

The functor $i_{P}^{G}$ thus identifies $A_{[L, \pi]}$ with $\bigotimes_{i} A_{\left[L_{i}, \pi_{i}\right]}$; since this isomorphism is induced by parabolic induction it agrees with the map $f$ of Theorem 12.3.

We now turn to the question of constructing natural elements of $A_{[L, \pi]}$. We will do this first in the case where the pair $(L, \pi)$ is simple. In this case, up to inertial equivalence, $L$ is isomorphic to $\mathrm{GL}_{n_{1}}(F)^{r}$ for some $n_{1}$ and $r$, and $\pi$ is a tensor power $\pi_{1}^{\otimes r}$ of an irreducible, supercuspidal $k$-representation $\pi_{1}$ of $\mathrm{GL}_{n_{1}}(F)$. Then, as in Section 9, we may choose a maximal distinguished supercuspidal $k$ type $\left(K_{1}, \tau_{1}\right)$ contained in $\pi_{1}$. Attached to this type are integers $f, f^{\prime}$ and, for each $m$ in $\left\{1, e_{q f^{\prime}}, \ell e_{q f^{\prime}}, \ell^{2} e_{q^{f^{\prime}}}, \ldots\right\}$, a maximal distinguished cuspidal type $\left(K_{m}, \tau_{m}\right)$; the representation $\tau_{m}$ factors as usual as $\kappa_{m} \otimes \sigma_{m}$. 
In this case, by definition, the projective $\mathcal{P}_{[L, \pi]}$ is given by:

$$
\mathcal{P}_{[L, \pi]} \cong \bigoplus_{\nu} i_{P_{v}}^{G} \bigotimes_{j} \mathcal{P}_{K_{v_{j}}, \tau_{v_{j}}}
$$

where $v$ runs over all increasing partitions $v_{1} \leqslant v_{2} \leqslant v_{3} \ldots$ of $r$ such that $v_{j}$ lies in $\left\{1, e_{q f^{\prime}}, \ell e_{q^{f^{\prime}}}, \ldots\right\}$ for all $j$, and $P_{v}$ is the standard (upper triangular) parabolic with block sizes corresponding to $v$.

Fix a $v$ as above, and let $t$ be its length. For $1 \leqslant i \leqslant r$, we can define an endomorphism $\Theta_{i, v}$ of $i_{P_{v}}^{G} \otimes_{j} \mathcal{P}_{L_{v_{j}}, \tau_{v_{j}}}$ by setting

$$
\Theta_{i, v}=\Sigma_{\mu} \bigotimes_{j} \Theta_{\mu_{j}, v_{j}},
$$

where $\mu$ runs over tuples $\left(\mu_{1}, \ldots, \mu_{s}\right)$ of nonnegative integers such that $\mu_{j} \leqslant v_{j}$ for all $j$, and the sum of the $\mu_{j}$ is $i$. (Here $\Theta_{\mu_{j}, v_{j}}$ is the endomorphism of $\mathcal{P}_{K_{v_{j}}, \tau_{v_{j}}}$ constructed in Theorem 9.4; the tensor product of these endomorphisms acts by functoriality of parabolic induction on $i_{P_{v}}^{G} \bigotimes_{j} \mathcal{P}_{K_{v_{j}}, \tau_{v_{j}}}$.)

Let $\Theta_{i}$ be the endomorphism of $\mathcal{P}_{[L, \pi]}$ that, for each $\nu$, acts via $\Theta_{i, v}$ on the summand $i_{P_{v}}^{G} \bigotimes_{j} \mathcal{P}_{K_{v_{j}}, \tau_{v_{j}}}$ of $\mathcal{P}_{[L, \pi]}$.

To understand the action of $\Theta_{i}$ on $\mathcal{P}_{[L, \pi]}$ we invoke results of Sections 9. In particular, we fix a lift $\tilde{\pi}_{1}$ of $\pi_{1}$ containing the type $\tilde{\kappa}_{1} \otimes \mathrm{St}_{s_{1}}$, where $s_{1}$ is the $\ell$-regular conjugacy class of $\mathrm{GL}_{n_{1} / e f}\left(\mathbb{F}_{q}\right)$ corresponding to $\sigma_{1}$. This choice gives rise to a compatible system of cuspidals $\left(M_{s}, \pi_{s}\right)$ as in Section 9; here $s$ varies over conjugacy classes in $\mathrm{GL}_{n_{1} i / e f}\left(\mathbb{F}_{q^{f}}\right)$, with $\ell$-regular part $\left(s_{1}\right)^{i}$, for $i \in\left\{1, e_{q f^{\prime}}, \ell e_{q^{f^{\prime}}}, \ldots\right\}$. As in the discussion before Theorem 9.4, we have an isomorphism:

$$
A_{M_{s}, \pi_{s}} \cong \overline{\mathcal{K}}\left[Z_{s}\right]^{W_{M_{s}}(s)} .
$$

For any $m$, the endomorphism ring $E_{K_{m}, \tau_{m}} \otimes \overline{\mathcal{K}}$ of $\mathcal{P}_{K_{m}, \tau_{m}} \otimes \overline{\mathcal{K}}$ factors as the product:

$$
\prod_{s} \overline{\mathcal{K}}\left[Z_{s}\right]^{W_{M_{s}}(s)}
$$

By Theorem 9.4, the endomorphism $\Theta_{i, m}$ of $\mathcal{P}_{K_{m}, \tau_{m}}$ maps to the element $\prod_{s} \theta_{i, s}$ under this identification, where $\theta_{i, s}$ is the element of $\overline{\mathcal{K}}\left[Z_{s}\right]$ constructed in Section 9.

Fix a $v$ of length $t$. We then have an isomorphism:

$$
\bigotimes_{j} E_{K_{v_{j}}, \tau_{v_{j}}} \otimes \overline{\mathcal{K}} \cong \prod_{\vec{s}} \bigotimes_{j} \overline{\mathcal{K}}\left[Z_{s_{j}}\right]^{W_{M_{s_{j}}}\left(s_{j}\right)}
$$


where $\vec{s}=s_{1}, \ldots, s_{t}$ is a sequence such that $s_{j}$ is a conjugacy class in $\mathrm{GL}_{n_{1} v_{j} / e f}\left(\mathbb{F}_{q^{f}}\right)$ with $\ell$-regular part $\left(s_{1}\right)^{v_{j}}$. If $s$ is the semisimple conjugacy class in $\mathrm{GL}_{n_{1} r / e f}\left(\mathbb{F}_{q^{f}}\right)$ corresponding to 'block diagonal' matrix with blocks $s_{1}, \ldots, s_{t}$, then $\overline{\mathcal{K}}\left[Z_{s}\right]$ is the tensor product of the $\overline{\mathcal{K}}\left[S_{j}\right]$. Moreover, under the composition

$$
\bigotimes_{j} E_{K_{v_{j}}, \tau_{v_{j}}} \rightarrow \bigotimes_{j} \overline{\mathcal{K}}\left[Z_{s_{j}}\right]^{W_{M_{s_{j}}}\left(s_{j}\right)} \hookrightarrow \overline{\mathcal{K}}\left[Z_{s}\right]
$$

the element $\Theta_{i, v}$ of $\bigotimes_{j} E_{K_{v_{j}}, \tau_{v_{j}}}$ maps to the element $\theta_{i, s}$.

In particular, action of $\Theta_{i, v}$ on the part of $i_{P_{\nu}}^{G} \bigotimes_{j} \mathcal{P}_{K_{v_{j}}, \tau_{n u_{j}}} \otimes \overline{\mathcal{K}}$ lying in the block $\left(M_{s}, \pi_{s}\right)$ is via the element of $A_{M_{s}, \pi_{s}}$ corresponding to $\theta_{i, s}$ under the isomorphism:

$$
A_{M_{s}, \pi_{s}} \cong \overline{\mathcal{K}}\left[Z_{s}\right]^{W_{M_{s}}(s)} .
$$

We immediately deduce:

THEOREM 12.5. The elements $\Theta_{1}, \ldots, \Theta_{r}$ lie in $A_{[L, \pi]}$.

Proof. The action of $\Theta_{i}$ on $\mathcal{P}_{[L, \pi]}$ coincides with the element $\prod_{M_{s}, \pi_{s}} \theta_{i, s}$ of the product $\prod_{M_{s}, \pi_{s}} A_{M_{s}, \pi_{s}}$; in particular it lies in the center of the endomorphism ring of $\mathcal{P}_{[L, \pi]}$.

We let $C_{[L, \pi]}$ be the $W(k)$-subalgebra of $A_{[L, \pi]}$ generated by the elements $\Theta_{i}, i \in 1, \ldots, r$, together with $\Theta_{r}^{-1}$. More generally, if $[L, \pi]$ is not simple but factors into the simple types $\left[L_{i}, \pi_{i}\right]$, we let $C_{[L, \pi]}$ be the tensor product of the algebras $C_{\left[L_{i}, \pi_{i}\right]}$, regarded as a subalgebra of $A_{[L, \pi]}$ via the isomorphism $A_{[L, \pi]} \cong \bigotimes_{i} A_{\left[L_{i}, \pi_{i}\right]}$ constructed above.

When $(L, \pi)$ is simple, it is not hard to show that the elements $\Theta_{1}, \ldots, \Theta_{r}$ are algebraically independent. Indeed, let $s$ be the semisimple conjugacy class in $\mathrm{GL}_{n / e f}\left(\mathbb{F}_{q^{f}}\right)$ given by $\left(s_{1}\right)^{r}$. Then $Z_{s}$ is free abelian group on $r$ generators, and $W_{M_{s}}(s)$ is the symmetric group that permutes them. The elements $\theta_{1, s}, \ldots$, $\theta_{r, s}$ of $W(k)\left[Z_{s}\right]$ are the elementary symmetric functions in these generators. In particular, they are algebraically independent (and generate $W(k)\left[Z_{s}\right]^{W_{M_{s}}(s)}$ over $W(k))$.

Since $\Theta_{i}$ maps to $\theta_{i, s}$ under the composition:

$$
C_{[L, \pi]} \hookrightarrow A_{[L, \pi]} \rightarrow A_{M_{s}, \pi_{s}} \cong \overline{\mathcal{K}}\left[Z_{s}\right]^{W_{M_{s}}(s)},
$$

we have:

Proposition 12.6. Let $s$ be the semisimple conjugacy class in $\mathrm{GL}_{n_{1} r / e f}\left(\mathbb{F}_{q^{f}}\right)$ given by $\left(s_{1}\right)^{r}$. Then the map $C_{[L, \pi]} \rightarrow W(k)\left[Z_{s}\right]^{W_{M_{s}}(s)}$ is an isomorphism. 
Proposition 12.7. The module $\mathcal{P}_{[L, \pi]}$ is an admissible $C_{[L, \pi]}[G]$-module (and hence is also admissible as an $A_{[L, \pi]}[G]$-module).

Proof. It suffices to show this in the case where $[L, \pi]$ is simple, and in this case it suffices to show that each $i_{P_{v}}^{G} \bigotimes_{j} \mathcal{P}_{K_{v_{j}}, \tau_{v_{j}}}$ is admissible over $C_{[L, \pi]}$. The module $\bigotimes_{j} \mathcal{P}_{K_{v_{j}}, \tau_{v_{j}}}$ is admissible over $\bigotimes_{j} C_{K_{v_{j}}, \tau_{v_{j}}}$.

It thus suffices to show that, for each $v$, the map $C_{[L, \pi]} \rightarrow \bigotimes_{j} C_{K_{v_{j}}, \tau_{v_{j}}}$ makes the latter into a finitely generated $C_{[L, \pi]}$-module. We have a commutative diagram:

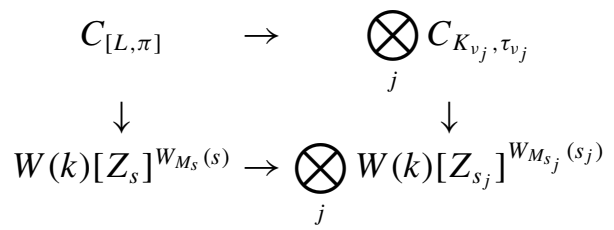

where $s$ is the semisimple conjugacy class in $\mathrm{GL}_{n_{1} r / e f}\left(\mathbb{F}_{q^{f}}\right)$ given by $\left(s_{1}\right)^{r}$ and $s_{j}$ is the semisimple conjugacy class in $\mathrm{GL}_{n_{1} v_{j} / e f}\left(\mathbb{F}_{q^{f}}\right)$ given by $\left(s_{1}\right)^{v_{j}}$. The vertical maps are isomorphisms. In particular, we have inclusions

$$
C_{[L, \pi]} \subseteq \bigotimes_{j} C_{K_{\mu_{j}}, \tau_{v_{j}}} \subseteq W(k)\left[Z_{s}\right]
$$

But $W(k)\left[Z_{s}\right]$ is finitely generated as a $C_{[L, \pi]}$-module, so the result follows.

We thus have:

THEOREM 12.8. The ring $A_{[L, \pi]}$ is a finitely generated, reduced, $\ell$-torsion free $W(k)$-algebra.

Proof. As $\mathcal{P}_{[L, \pi]}$ is admissible over $C_{[L, \pi]}$ its endomorphism ring is finitely generated as a $C_{[L, \pi]}$-module. Thus $A_{[L, \pi]}$ is a finitely generated $C_{[L, \pi]}$-module. Since $C_{[L, \pi]}$ is a polynomial ring over $W(k)$, it is immediate that $A_{[L, \pi]}$ is a finitely generated $W(k)$-algebra. The fact that $A_{[L, \pi]}$ is $\ell$-torsion free follows from $A_{[L, \pi]} \subset \operatorname{End}_{W(k)[G]}\left(\mathcal{P}_{[L, \pi]}\right)$ and the fact that $\mathcal{P}_{[L, \pi]}$ is $\ell$-torsion free. Reducedness follows from the fact that $A_{[L, \pi]}$ embeds in $A_{[L, \pi]} \otimes_{W(k)} \overline{\mathcal{K}}$, and the latter is reduced by Proposition 12.1 .

THEOREM 12.9. Let $\Pi$ be an object of $\operatorname{Rep}_{W(k)}(G)_{[L, \pi]}$ that is finitely generated as a $W(k)[G]$-module. Then $\Pi$ is an admissible $A_{L, \pi}[G]$-module.

Proof. As $\mathcal{P}_{[L, \pi]}$ is faithfully projective, there is a surjection of a direct sum of (possibly infinitely many) copies of $\mathcal{P}_{[L, \pi]}$ onto $\Pi$. Any element $x$ of $\Pi$ is in the 
image of this surjection, and any element $\tilde{x}$ that maps to $x$ is nonzero only in finitely many copies of $\mathcal{P}_{[L, \pi]}$. Thus if $\Pi$ is generated by finitely many elements, then there is a finite direct sum of copies of $\mathcal{P}_{[L, \pi]}$ whose image in $\Pi$ contains all the generators, and this direct sum surjects onto $\Pi$.

We next turn to the question of understanding the $k$-points of $\operatorname{Spec} A_{[L, \pi]}$. If $\Pi$ is an irreducible object in $\operatorname{Rep}_{k}(G)_{[L, \pi]}$, then it admits an action of $A_{[L, \pi]}$; by Schur's lemma this action is via a map $f_{\Pi}: A_{[L, \pi]} \rightarrow k$. Conversely, if $f:$ $A_{[L, \pi]} \rightarrow k$ is any map, then $\mathcal{P}_{[L, \pi]} \otimes_{A_{[L, \pi]}, f} k$ is an admissible smooth $k[G]$-module on which $A_{[L, \pi]}$ acts via $f$, so there exists an irreducible $\Pi$ such that $f=f_{\Pi}$.

There is thus a surjection $\Pi \mapsto f_{\Pi}$ from isomorphism classes of irreducible objects in $\operatorname{Rep}_{k}(G)_{[L, \pi]}$ to $k$-points of $\operatorname{Spec} A_{[L, \pi]}$.

LEMMA 12.10. Suppose $\Pi$ and $\Pi^{\prime}$ are irreducible $k$-representations in $\operatorname{Rep}_{k}(G)_{[L, \pi]}$ with the same supercuspidal support. Then $f_{\Pi}=f_{\Pi^{\prime}}$.

Proof. Suppose that $\Pi$ and $\Pi^{\prime}$ both have supercuspidal support $\left(L, \pi^{\prime}\right)$ for some $\pi^{\prime}$ inertially equivalent to $\pi$. Let $\tilde{\pi}^{\prime}$ be a lift of $\pi^{\prime}$ to $\overline{\mathcal{K}}$. Then both $\Pi$ and $\Pi^{\prime}$ are subquotients of $i_{P}^{G} \tilde{\pi}$. On the other hand the action of $A_{[L, \pi]}$ on $i_{P}^{G} \tilde{\pi}$ factors through $A_{[L, \pi]} \otimes \overline{\mathcal{K}}$, and the latter acts on $i_{P}^{G} \tilde{\pi}$ by scalars. It follows that any element of $A_{[L, \pi]}$ acts on $\Pi$ and $\Pi^{\prime}$ by the same scalar; that is, $f_{\Pi}=f_{\Pi^{\prime}}$.

Conversely, we have:

Proposition 12.11. Let $\Pi$ and $\Pi^{\prime}$ be irreducible $k$-representations in $\operatorname{Rep}_{k}(G)_{[L, \pi]}$ and suppose that $f_{\Pi}$ and $f_{\Pi^{\prime}}$ agree on $C_{[L, \pi]}$. Then $\Pi$ and $\Pi^{\prime}$ have the same supercuspidal support.

Proof. It suffices to prove this in the case where $(L, \pi)$ is simple. The supercuspidal supports of $\Pi$ and $\Pi^{\prime}$ have the form $(L, \pi \otimes \chi)$ and $\left(L, \pi \otimes \chi^{\prime}\right)$, respectively, where $\chi$ and $\chi^{\prime}$ are unramified characters of $L$.

Choose a lift $\tilde{\chi}$ of $\chi$ to a character $L / L_{0} \rightarrow W(k)^{\times}$, and consider the pair $(L, \tilde{\pi} \otimes \tilde{\chi})$, where $\tilde{\pi}$ is the representation $\tilde{\pi}_{1}^{\otimes r}$ of $L$.

If $P$ is a parabolic with Levi $L$, then $A_{[L, \pi]}$ acts on $i_{P}^{G} \tilde{\pi} \otimes \tilde{\chi}$ via the map

$$
A_{[L, \pi]} \rightarrow A_{M_{s}, \pi_{s}} \cong\left(\overline{\mathcal{K}}\left[\left(L / L_{0}\right)\right]^{H_{s}}\right)^{W_{M_{s}}(s)} \cong \overline{\mathcal{K}}\left[Z_{s}\right]^{W_{M_{s}}(s)},
$$

where $s$ is the semisimple conjugacy class $\left(s_{1}\right)^{r}$ in $\mathrm{GL}_{n_{1} r / e f}\left(\mathbb{F}_{q^{f}}\right)$. The action of an element of $A_{[L, \pi]}$ on $i_{P}^{G} \tilde{\pi} \otimes \tilde{\chi}$ may be described as follows: the character $\tilde{\chi}$ can be viewed as a $W(k)$-point of $\operatorname{Spec} \overline{\mathcal{K}}\left[L / L_{0}\right]$; then an element $x$ of $\left(\overline{\mathcal{K}}\left[\left(L / L_{0}\right)\right]^{H_{s}}\right)^{W_{M_{s}}(s)}$ acts on $i_{P}^{G} \tilde{\pi} \otimes \tilde{\chi}$ by the scalar $x(\tilde{\chi})$, where we regard $x$ as a function on $\operatorname{Spec} \overline{\mathcal{K}}\left[L / L_{0}\right]$. 
Reducing $\bmod \ell$, we find that an element of $A_{[L, \pi]}$ that maps to a given element $x$ of $\left(W(k)\left[\left(L / L_{0}\right)\right]^{H_{s}}\right)^{W_{M_{s}}(s)}$ acts on $i_{P}^{G} \pi \otimes \chi$ (and hence on $\Pi$ ) by the scalar $x(\chi)$. Similarly, such an element acts by $x\left(\chi^{\prime}\right)$ on $\Pi^{\prime}$.

Since $f_{\Pi}$ and $f_{\Pi^{\prime}}$ agree on $C_{[L, \pi]}$, we have $\theta_{i, s}(\chi)=\theta_{i, s}\left(\chi^{\prime}\right)$ for all $i$. Thus, when considered as $k$-points of $\operatorname{Spec} W(k)\left[L / L_{0}\right]$, the characters $\chi$ and $\chi^{\prime}$ map to the same $k$-point of $\left(\operatorname{Spec} W(k)\left[\left(L / L_{0}\right)\right]^{H_{s}}\right)^{W_{M_{s}}(s)}$. In particular, the pairs $(L, \pi \otimes \chi)$ and $\left(L, \pi \otimes \chi^{\prime}\right)$ are conjugate, so $\Pi$ and $\Pi^{\prime}$ have the same supercuspidal support as claimed.

Combining these, we have:

COROLlARY 12.12. If $\Pi$ and $\Pi^{\prime}$ are irreducible representations of $G$ over $k$ that lie in $\operatorname{Rep}_{W(k)}(G)_{[L, \pi]}$, and $f_{\Pi}, f_{\Pi^{\prime}}$ are the maps $A_{[L, \pi]} \rightarrow k$ giving the action of $A_{L, \pi}$ on $\Pi$ and $\Pi^{\prime}$ respectively, then $f_{\Pi}=f_{\Pi^{\prime}}$ if, and only if, $\Pi$ and $\Pi^{\prime}$ have the same supercuspidal supports. Moreover, the map $\operatorname{Spec} A_{[L, \pi]} \otimes k \rightarrow$ $\operatorname{Spec} C_{[L, \pi]} \otimes k$ is a bijection on k-points.

COROLlaRY 12.13. The composition:

$$
C_{[L, \pi]} \otimes k \rightarrow A_{[L, \pi]} \otimes k \rightarrow\left(A_{[L, \pi]} \otimes k\right)^{\mathrm{red}}
$$

identifies $C_{[L, \pi]} \otimes k$ with the reduced quotient of $A_{[L, \pi]} \otimes k$.

Proof. The map $A_{[L, \pi]} \rightarrow \overline{\mathcal{K}}\left[Z_{s}\right]^{W_{M_{s}}(s)}$ factors through $W(k)\left[Z_{s}\right]^{W_{M_{s}}(s)}$, where $s$ is the semisimple conjugacy class in $\mathrm{GL}_{n_{1} r / e f}\left(\mathbb{F}_{q^{f}}\right)$ given by $\left(s_{1}\right)^{r}$, and the composition:

$$
C_{[L, \pi]} \otimes k \rightarrow A_{[L, \pi]} \otimes k \rightarrow k\left[Z_{s}\right]^{W_{M_{s}}(s)}
$$

is an isomorphism. We thus obtain a map $\left(A_{[L, \pi]} \otimes k\right)^{\mathrm{red}} \rightarrow C_{[L, \pi]} \otimes k$ that is left inverse to the map $C_{[L, \pi]} \otimes k \rightarrow\left(A_{[L, \pi]} \otimes k\right)^{\text {red }}$. We must show that it is also a right inverse. For this it suffices to show that the map $\left(A_{[L, \pi]} \otimes k\right)^{\mathrm{red}} \rightarrow C_{[L, \pi]} \otimes k$ is injective. But both maps are bijections on $k$-points. Since Spec $C_{[L, \pi]}$ is integral, so is $\operatorname{Spec}\left(A_{[L, \pi]} \otimes k\right)^{\text {red }}$; the map $\operatorname{Spec} C_{[L, \pi]} \otimes k \rightarrow \operatorname{Spec}\left(A_{[L, \pi]} \otimes k\right)^{\text {red }}$ is dominant and so the induced map on rings of functions must be injective.

\section{Further results}

In this section we establish more precise results on the structure of $A_{[L, \pi]}$; we focus on the case in which the pair $(L, \pi)$ is simple. Fix an irreducible supercuspidal representation $\pi_{1}$ of $\mathrm{GL}_{n}(F)$, and for each positive integer $m$, let $G_{m}$ be the group $\mathrm{GL}_{n m}(F)$, let $L_{m}$ be a Levi of $G_{m}$ isomorphic to a product of $m$ 
copies of $\mathrm{GL}_{n}(F)$, and let $\pi_{m}$ be the representation $\left(\pi_{1}\right)^{\otimes m}$ of $L_{m}$, so that $\left(L_{m}, \pi_{m}\right)$ is a simple pair.

All of our results in this section rely on the following result, which is proved in [H1]:

THEOREM 13.1 [H1, Proposition 4.9]. Let $(K, \tau)$ be a maximal distinguished cuspidal $k$-type of $G_{m}$, and let $\mathfrak{p}$ be a prime ideal of $E_{K, \tau}$ with residue field $\kappa(\mathfrak{p})$. Then the cosocle of the tensor product $\mathcal{P}_{K, \tau} \otimes_{E_{K, \tau}} \kappa(\mathfrak{p})$ is absolutely irreducible and generic.

Suppose the inertial supercuspidal support of $(K, \tau)$ is $\left(L_{m}, \pi_{m}\right)$, so that there is an action of $A_{L_{m}, \pi_{m}}$ on $\mathcal{P}_{K, \tau}$ and a corresponding injection $A_{L_{m}, \pi_{m}} \rightarrow E_{K, \tau}$. Let $\mathfrak{m}$ be a maximal ideal of $A_{L_{m}, \pi_{m}}$, with residue field $k$. We then have:

LEMMA 13.2. Every irreducible quotient of $\mathcal{P}_{K, \tau} / \mathfrak{m} \mathcal{P}_{K, \tau}$ is generic.

Proof. The quotient $E_{K, \tau} / \mathfrak{m} E_{K, \tau}$ is a finite-dimensional $k$-algebra, and is thus a product of finite-dimensional local $k$-algebras $E_{i}$, with maximal ideals $\mathfrak{m}_{i}$. We then have an isomorphism:

$$
\mathcal{P}_{K, \tau} / \mathfrak{m} \mathcal{P}_{K, \tau} \cong \bigoplus_{i} \mathcal{P}_{K, \tau} \otimes_{E_{K, \tau}} E_{i}
$$

and it suffices to show that any irreducible quotient of the right-hand side is generic.

Fix $i$. Then $E_{i}$ admits a filtration by ideals

$$
0=I_{0} \subseteq I_{1} \subseteq \cdots \subseteq I_{r}=E_{i}
$$

such that the quotients $I_{j+1} / I_{j}$ are one-dimensional $k$-vector spaces. We can thus choose, for each $j$, an element $x_{j}$ of $I_{j}$ such that $x_{j}$ generates $I_{j}$ modulo $I_{j-1}$. Then multiplication by $x_{j}$ induces an surjection of $\mathcal{P}_{K, \tau} \otimes_{E_{K, \tau}} \kappa\left(\mathfrak{m}_{i}\right)$ onto $I_{j} \mathcal{P}_{K, \tau} / I_{j-1} \mathcal{P}_{K, \tau}$. It follows that $\mathcal{P}_{K, \tau} \otimes_{E_{K, \tau}} E_{i}$ admits a filtration such that the cosocle of each successive quotient is irreducible and generic. Thus every irreducible quotient of $\mathcal{P}_{K, \tau} \otimes_{E_{K, \tau}} E_{i}$ is generic as claimed.

We will say a simple object $\Pi$ of $\operatorname{Rep}_{W(k)}\left(G_{m}\right)_{\left[L_{m}, \pi_{m}\right]}$ is generic if either:

- $\ell \Pi=0$, and $\Pi$ is an irreducible generic $k$-representation of $G_{m}$; or

- $\ell \Pi=\Pi$, and $\Pi \otimes_{\mathcal{K}} \overline{\mathcal{K}}$ is a direct sum of irreducible $\overline{\mathcal{K}}$-representations of $G_{m}$, each of which is generic. 
We can then rephrase Theorem 13.1 as follows:

THEOREM 13.3. Let $(K, \tau)$ be a maximal distinguished cuspidal $k$-type of $G_{m}$, with supercuspidal support $\left(L_{m}, \pi_{m}\right)$. Then every simple quotient of $\mathcal{P}_{K, \tau}$ is generic. Moreover, every simple generic object of $\operatorname{Rep}_{W(k)}(G)_{\left[L_{m}, \pi_{m}\right]}$ is a quotient of $\mathcal{P}_{K, \tau}$.

Proof. Let $\Pi$ be a simple quotient of $\mathcal{P}_{K, \tau}$. Then $\operatorname{End}_{W(k)[G]}(\Pi)$ is either isomorphic to $k$, if $\ell \Pi=0$, or to an extension $\mathcal{K}^{\prime}$ of $\mathcal{K}$ (note that every endomorphism of $\Pi$ lifts to an endomorphism of $\mathcal{P}_{K, \tau}$, so that $\Pi$ has a commutative endomorphism ring).

Suppose first that $\operatorname{End}_{W(k)[G]}(\Pi)$ is an extension $\mathcal{K}^{\prime}$ of $\mathcal{K}$. Then, since $A_{L_{m}, \pi_{m}}$ acts on $\Pi$, and the natural map $A_{L_{m}, \pi_{m}} \rightarrow E_{K, \tau}$ is an isomorphism after inverting $\ell$, we have a map $E_{K_{m}, \tau_{m}} \rightarrow \operatorname{End}_{W(k)[G]}(\Pi)$. This map is surjective because every endomorphism of $\Pi$ lifts to an element of $E_{K_{m}, \tau_{m}}$, and hence its kernel is a maximal ideal $\mathfrak{m}$. Then $\Pi$ then a quotient of $\mathcal{P}_{K, \tau} \otimes_{E_{K, \tau}} \kappa(\mathfrak{m})$. Since $\Pi$ is simple, the cosocle of $\mathcal{P}_{K, \tau} \otimes_{E_{K, \tau}} \kappa(\mathfrak{m})$ surjects onto $\Pi$. It follows that, when regarded as a representation over $\kappa(\mathfrak{m}), \Pi$ is absolutely irreducible and generic, so that $\Pi$ is a generic $W(k)[G]$-module as claimed.

On the other hand, if $\ell \Pi=0$, then the annihilator of $\Pi$ in $A_{L_{m}, \pi_{m}}$ is a maximal ideal $\mathfrak{m}$ with residue field $k$, and $\Pi$ is an irreducible quotient of $\mathcal{P}_{K, \tau} / \mathfrak{m} \mathcal{P}_{K, \tau}$, and is therefore generic by Lemma 13.2.

Conversely, let $\Pi$ be a simple generic object in $\operatorname{Rep}_{W(k)}(G)_{\left[L_{m}, \pi_{m}\right]}$, and set $\kappa=k$ if $\ell \Pi=0, \kappa=\overline{\mathcal{K}}$ otherwise. Then $\Pi \otimes \kappa$ is a direct sum of irreducible representations; let $\Pi^{\prime}$ be an irreducible summand. The action of $A_{\left[L_{m}, \pi_{m}\right]}$ on $\Pi^{\prime}$ is via a map $A_{\left[L_{m}, \pi_{m}\right]} \rightarrow \kappa$. This map depends on the choice of $\Pi^{\prime}$, but its kernel $\mathfrak{m}$ does not.

Let $\tilde{\mathfrak{m}}$ be a maximal ideal of $E_{K, \tau}$ lying over $\mathfrak{m}$. Since $\kappa$ is algebraically closed, and $E_{K, \tau}$ is finite over $A_{\left[L_{m}, \pi_{m}\right]}$, the map $A_{\left[L_{m}, \pi_{m}\right]} \rightarrow \kappa$ giving the action of $A_{\left[L_{m}, \pi_{m}\right]}$ on $\Pi^{\prime}$ extends to a map $f: E_{K, \tau} / \tilde{\mathfrak{m}} \rightarrow \kappa$.

The cosocle of $\Pi^{\prime \prime}$ of $\mathcal{P}_{K, \tau} \otimes_{E_{K, \tau}, f} \kappa$ is an irreducible generic representation on which $A_{\left[L_{m}, \pi_{m}\right]}$ acts via $f$; in particular the supercuspidal supports of $\Pi^{\prime \prime}$ and $\Pi^{\prime}$ coincide. Since there is a unique generic representation with given supercuspidal support, $\Pi^{\prime \prime}$ is isomorphic to $\Pi^{\prime}$; we thus obtain a surjection $\mathcal{P}_{K, \tau} \otimes_{E_{K, \tau}, f} \kappa \rightarrow \Pi^{\prime}$. In particular, $\operatorname{Hom}_{W(k)[G]}\left(\mathcal{P}_{K, \tau} \otimes_{E_{K, \tau}, f} \kappa, \Pi \otimes \kappa\right)$ is nonzero and so $\operatorname{Hom}_{W(k)[G]}\left(\mathcal{P}_{K, \tau}, \Pi\right)$ is nonzero as well.

We will apply this result in the setting of Section 9. Fix a maximal distinguished supercuspidal $k$-type $\left(K_{1}, \tau_{1}\right)$ containing $\pi_{1}$; then the construction of Section 9 gives rise to maximal distinguished cuspidal $k$-types $\left(K_{m}, \tau_{m}\right)$ for $m$ in 
$\left\{1, e_{q^{f^{\prime}}}, \ell e_{q^{f^{\prime}}}, \ldots\right\}$. Fix an element $m>1$ in this set, and let $m^{\prime}$ be the largest element of this set strictly smaller than $m$. Set $j=m / m^{\prime}$. We then have projectives $\mathcal{P}_{m}=\mathcal{P}_{K_{m}, \tau_{m}}$ and $\mathcal{P}_{m^{\prime}}=\mathcal{P}_{K_{m^{\prime}}, \tau_{m^{\prime}}}$; we denote their endomorphism rings by $E_{m}$ and $E_{m^{\prime}}$, respectively.

Let $M$ be a Levi of $G_{m}$ containing $L_{m}$ and isomorphic to a product of $j$ copies of $\operatorname{GL}_{n m^{\prime}}(F)$, and let $P$ be a parabolic with Levi $M$. Theorem 13.3 gives us the following relationship between the $W(k)[M]$-module $\mathcal{P}_{m^{\prime}}^{\otimes j}$ and the parabolic restriction $r_{G}^{P} \mathcal{P}_{m}$ :

PROPOSITION 13.4. The map:

$$
r_{G}^{P} \mathcal{P}_{m} \otimes \operatorname{Hom}_{W(k)[M]}\left(r_{G}^{P} \mathcal{P}_{m}, \mathcal{P}_{m^{\prime}}^{\otimes j}\right) \rightarrow \mathcal{P}_{m^{\prime}}^{\otimes j}
$$

is surjective.

Proof. Note that $r_{P}^{G} \mathcal{P}_{m}$ is a projective $W(k)[M]$-module. It thus suffices to show that every simple quotient of $\mathcal{P}_{m^{\prime}}^{\otimes j}$ admits a map from $r_{P}^{G} \mathcal{P}_{m}$. By Theorem 13.3, any simple quotient $\Pi \mathcal{P}_{m^{\prime}}^{\otimes j}$ is generic and lies in $\operatorname{Rep}_{W(k)}(M)_{\left[L_{m}, \pi_{m}\right]}$. Thus $i_{P}^{G} \Pi$ lies in $\operatorname{Rep}_{W(k)}(G)_{\left[L_{m}, \pi_{m}\right]}$ and has a simple generic subquotient. Hence $\operatorname{Hom}_{W(k)[G]}\left(\mathcal{P}_{m}\right.$, $\left.i_{P}^{G} \Pi\right)$ is nonzero, and so $\operatorname{Hom}_{W(k)[M]}\left(r_{P}^{G} \mathcal{P}_{m}, \Pi\right)$ is nonzero as well.

As a consequence, we deduce:

THEOREM 13.5. There exists a unique map: $f_{m}: E_{m} \rightarrow E_{m^{\prime}}^{\otimes j}$ making the diagram:

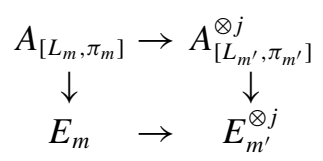

commute.

Proof. Our descriptions of $A_{\left[L_{m}, \pi_{m}\right]} \otimes \overline{\mathcal{K}}$ and $E_{m} \otimes \overline{\mathcal{K}}$ show that the natural map $A_{\left[L_{m}, \pi_{m}\right]} \rightarrow E_{m}$ giving the action of $A_{\left[L_{m}, \pi_{m}\right]}$ on $\mathcal{P}_{m}$ becomes an isomorphism after tensoring with $\overline{\mathcal{K}}$, and hence after inverting $\ell$. Uniqueness of the map is clear thus if such a map exists.

To construct the map, note that $E_{m}$ acts on $\mathcal{P}_{m}$ and hence on $r_{G}^{P} \mathcal{P}_{m}$. Moreover, if $x$ is an element of $E_{m}$ in the image of $A_{\left[L_{m}, \pi_{m}\right]}$, then $x$ acts on $r_{G}^{P} \mathcal{P}_{m}$ via the natural map $A_{\left[L_{m}, \pi_{m}\right]} \rightarrow A_{\left[L_{m^{\prime}}, \pi_{m^{\prime}}\right]}^{\otimes j}$, by the adjointness of parabolic induction and restriction. It thus suffices to show that the action of $E_{m}$ preserves the kernel of the surjection:

$$
r_{G}^{P} \mathcal{P}_{m} \otimes \operatorname{Hom}_{W(k)[M]}\left(r_{G}^{P} \mathcal{P}_{m}, \mathcal{P}_{m^{\prime}}^{\otimes j}\right) \rightarrow \mathcal{P}_{m^{\prime}}^{\otimes j}
$$


On the other hand, if $x$ lies in $E_{m}$, then for some $a, \ell^{a} x$ lies in $A_{\left[L_{m}, \pi_{m}\right]}$, and hence acts via an element of $A_{\left[L_{m^{\prime}}, \pi_{m^{\prime}}\right]}^{\otimes j}$ on $r_{G}^{P} \mathcal{P}_{m}$. In particular, $\ell^{a} x$ preserves the kernel of the map:

$$
\left[r_{G}^{P} \mathcal{P}_{m} \otimes \operatorname{Hom}_{W(k)[M]}\left(r_{G}^{P} \mathcal{P}_{m}, \mathcal{P}_{m^{\prime}}^{\otimes j}\right)\right] \rightarrow \mathcal{P}_{m^{\prime}}^{\otimes j}
$$

and so $x$ preserves this kernel as well (as the kernel contains all $\ell$-torsion). Thus $x$ gives an endomorphism of $\mathcal{P}_{m^{\prime}}^{\otimes j}$ and we are done.

We have the following control over the image of $E_{m}$ in $E_{m^{\prime}}^{\otimes j}$ :

THEOREM 13.6. Let $y$ be an element of $E_{m^{\prime}}^{\otimes j}$ such that, for some a, $\ell^{a} y$ lies in the image of $E_{m}$. Then there exist elements $x_{1}, x_{2}$ of $E_{m}$, and a positive integer $b$, such that:

(1) $x_{2}$ is supported on double cosets of the form $K_{m} z_{m, m}^{i} K_{m}$; and

(2) $f_{m}\left(x_{2}\right)=\ell^{b}\left(y-f_{m}\left(x_{1}\right)\right)$.

Proof. We invoke results from Section 9. In particular, by Proposition 9.6 we have a support-preserving map

$$
E_{m} / I^{\text {cusp }} \hookrightarrow H\left(G_{m}, K_{m}, \tilde{\kappa}_{m} \otimes \mathcal{P}_{\sigma_{m}}^{\prime}\right)
$$

that is an isomorphism after inverting $\ell$ and whose cokernel is supported on double cosets of the form $K_{m} z_{m, m}^{i} K_{m}$.

The $G$-cover construction of Section 9 yields a map:

$$
T: E_{m^{\prime}}^{\otimes j} \rightarrow H\left(G_{m}, K_{m}, \tilde{\kappa}_{m} \otimes i_{\bar{P}}^{\bar{G}_{m}} \mathcal{P}_{\bar{M}}\right)
$$

and Lemma 9.7 shows that central elements of $H\left(G_{m}, K_{m}, \tilde{\kappa}_{m} \otimes i{\overline{\bar{G}_{m}}}_{\bar{P}} \mathcal{P}_{\bar{M}}\right)$ descend to elements of $H\left(G_{m}, K_{m}, \tilde{\kappa}_{m} \otimes \mathcal{P}_{\sigma_{m}}^{\prime}\right)$.

If $\ell^{a} y$ is in the image of $E_{m}$, then for some $c \geqslant a, \ell^{c} y$ lies in the image of $A_{\left[L_{m}, \pi_{m}\right]}$. In particular, $T\left(\ell^{c} y\right)$ coincides with an element of $A_{\left[L_{m}, \pi_{m}\right]}$ as an endomorphism of c-Ind $K_{K_{m}}^{G_{m}} \tilde{\kappa}_{m} \otimes i \bar{G}_{\bar{P}} \mathcal{P}_{\bar{M}}$ and is thus central. In particular, $T y$ is central as well.

We thus obtain an element $y^{\prime}$ of $H\left(G_{m}, K_{m}, \tilde{\kappa}_{m} \otimes \mathcal{P}_{\sigma_{m}}^{\prime}\right)$ corresponding to $T y$. Let $y_{2}^{\prime}$ be the part of $y^{\prime}$ supported on double cosets of the form $K_{m} z_{m, m}^{i} K_{m}$, and set $y_{1}^{\prime}=y-y_{2}^{\prime}$. Then $y_{1}^{\prime}$ is the image of an element $x_{1}$ of $E_{m}$. Moreover, for some $b$, $\ell^{b} y_{2}^{\prime}$ is the image of an element $x_{2}$ of $E_{m}$ supported on double cosets of the form $K_{m} z_{m, m}^{i} K_{m}$.

The action of $T f_{m}\left(x_{2}\right)$ on c- $\operatorname{Ind}_{K_{m}}^{G_{m}} \tilde{\kappa}_{m} \otimes \mathcal{P}_{\sigma_{m}}^{\prime}$ then coincides with that of $\ell^{b} y_{2}^{\prime}$, and that of $T f_{m}\left(x_{1}\right)$ coincides with $y_{1}^{\prime}$. We thus have $T f_{m}\left(x_{2}\right)=\ell^{b}\left(T y-T f_{m}\left(x_{1}\right)\right)$, and since $T$ is injective the claim follows. 
The existence of maps $f_{m}$ as above allows us to considerably simplify our description of $A_{\left[L_{m}, \pi_{m}\right]}$. From this point on we let $m$ be arbitrary. Call a partition $v$ of $m$ admissible if each $v_{j}$ lies in the set $\left\{1, e_{q^{f^{\prime}}}, \ell e_{q f^{\prime}}, \ldots\right\}$. We order the admissible partitions by refinement, via the convention $v \preceq v^{\prime}$ if $v$ refines $v^{\prime}$. Since each element of the set $\left\{1, e_{q^{f^{\prime}}}, \ell e_{q^{f^{\prime}}}, \ldots\right\}$ divides the next, there is a unique maximal (that is, coarsest) partition $v$ of $m$ for every $m$.

For any admissible partition $v$ of $m$ we have a map

$$
A_{\left[L_{m}, \pi_{m}\right]} \rightarrow \bigotimes_{j} A_{L_{v_{j}}, \pi_{v_{j}}} \rightarrow \bigotimes_{j} E_{v_{j}}
$$

THEOREM 13.7. Let $v$ be the maximal admissible partition of $m$. Then the map

$$
A_{\left[L_{m}, \pi_{m}\right]} \rightarrow \bigotimes_{j} E_{v_{j}}
$$

is injective, and has saturated image (that is, if $\ell^{a} x$ lies in the image for some $a$, then so does $x$ ). In particular, if $m$ lies in $\left\{1, e_{q^{f^{\prime}}}, \ell e_{q^{f^{\prime}}}, \ldots\right\}$ then the map $A_{\left[L_{m}, \pi_{m}\right]} \rightarrow E_{m}$ is an isomorphism.

Proof. We have seen that $A_{\left[L_{m}, \pi_{m}\right]}$ is the intersection, in $\operatorname{End}_{W(k)\left[G_{m}\right]}\left(\mathcal{P}_{\left[L_{m}, \pi_{m}\right]} \otimes \mathcal{K}\right)$, of $A_{\left[L_{m}, \pi_{m}\right]} \otimes \mathcal{K}$ and $\operatorname{End}_{W(k)\left[G_{m}\right]}\left(\mathcal{P}_{\left[L_{m}, \pi_{m}\right]}\right)$.

Let $x$ be an element of $\bigotimes_{j} E_{v_{j}}$ such that $\ell^{a} x$ is the image of $y \in A_{\left[L_{m}, \pi_{m}\right]}$. For any admissible partition $v^{\prime}$ of $m, v^{\prime}$ refines $v$. We thus have a map:

$$
\bigotimes_{j} E_{v_{j}} \rightarrow \bigotimes_{i} E_{v_{i}^{\prime}}
$$

obtained by iteratively applying maps of the form $f_{v_{j}}$ to the tensor factors. For each $v^{\prime}$, let $x_{v^{\prime}}$ be the image of $x$ in $\bigotimes_{i} E_{v_{i}^{\prime}}$. Then $x_{v^{\prime}}$ gives an endomorphism of $i_{P_{v^{\prime}}}^{G_{m}} \bigotimes_{i} \mathcal{P}_{v_{i}^{\prime}}$, and the action of $\ell^{a} x_{v^{\prime}}$ on this space coincides with that of $y$.

Now $\mathcal{P}_{\left[L_{m}, \pi_{m}\right]}$ is isomorphic to the direct sum of the spaces $i_{P_{v^{\prime}}}^{G_{m}} \bigotimes_{i} \mathcal{P}_{v_{i}^{\prime}}$ as $v^{\prime}$ varies over all admissible partitions. The endomorphism of $\mathcal{P}_{\left[L_{m}, \pi_{m}\right]}$ that acts by $x_{v^{\prime}}$ on the summand corresponding to $v^{\prime}$ for all $v^{\prime}$ is given by $y / \ell^{a}$ and thus lies in $A_{\left[L_{m}, \pi_{m}\right]}$.

We conclude by giving a characterization of the image of $A_{\left[L_{m}, \pi_{m}\right]}$ in $\bigotimes_{j} E_{v_{j}}$, where $v$ remains the maximal admissible partition of $m$. We have $\tau_{1}=\kappa_{1} \otimes \sigma_{1}$, where $\sigma_{1}$ is a supercuspidal representation of $\bar{G}_{1}$ corresponding to an $\ell$-regular semisimple conjugacy class $s^{\prime}$ in $\bar{G}_{1}$. We have an isomorphism:

$$
E_{v_{j}} \otimes \overline{\mathcal{K}} \rightarrow \prod_{s} \overline{\mathcal{K}}\left[Z_{s}\right]^{W_{M_{s}}(s)},
$$


where $s$ runs over semisimple conjugacy classes in $G_{v_{j}}$ with $\ell$-regular part $\left(s^{\prime}\right)^{v_{j}}$. For such an $s$, let $\phi_{s}: E_{v_{j}} \rightarrow \overline{\mathcal{K}}\left[Z_{s}\right]^{W_{M_{s}}(s)}$ be the projection onto the corresponding factor.

Let $\vec{s}$ be a sequence of conjugacy classes, where $\vec{s}_{j}$ is a semisimple conjugacy class in $G_{v_{j}}$ with $\ell$-regular part $\left(s^{\prime}\right)^{v_{j}}$. The tensor product of the $\phi_{\vec{s}_{j}}$ gives a map:

$$
\phi_{\vec{s}}: \bigotimes_{j} E_{v_{j}} \rightarrow \bigotimes_{j} \overline{\mathcal{K}}\left[Z_{\vec{s}_{j}}\right]^{W_{M_{\vec{s}_{j}}}\left(\vec{s}_{j}\right)} .
$$

We may also regard $\vec{s}$ as a single conjugacy class in $M_{v}$, where $M_{v}$ is a Levi subgroup of $G_{m}$ with 'block decomposition' given by $\nu$.

Now suppose that $\vec{s}$ and $\vec{s}^{\prime}$ are two conjugacy classes in $M_{v}$ as above, and let $w \in W\left(G_{m}\right)$ be an element of $W\left(G_{m}\right)$ that conjugates $\vec{s}$ to $\vec{s}^{\prime}$. Then conjugation by $w$ induces an isomorphism:

$$
\prod_{j} Z_{\bar{s}_{j}} \cong \prod_{j} Z_{\bar{s}_{j}^{\prime}}
$$

and a compatible isomorphism:

$$
\prod_{j} W_{M_{\vec{s}_{j}}}\left(\vec{s}_{j}\right) \cong \prod_{j} W_{M_{\vec{s}_{j}^{\prime}}}\left(\vec{s}_{j}^{\prime}\right)
$$

where we regard all the products as subgroups of $M_{v}$, and hence of $G_{m}$. We thus obtain isomorphisms:

$$
\psi_{w}: \bigotimes_{j} \overline{\mathcal{K}}\left[Z_{\vec{s}_{j}}\right]^{W_{M_{\vec{s}_{j}}}\left(\vec{s}_{j}\right)} \cong \bigotimes_{j} \overline{\mathcal{K}}\left[Z_{\vec{s}_{j}^{\prime}}\right]^{W_{\vec{s}_{j}^{\prime}}\left(\vec{s}_{j}^{\prime}\right)} .
$$

THEOREM 13.8. An element $x$ of $\bigotimes_{j} E_{v_{j}}$ lies in the image of $A_{\left[L_{m}, \pi_{m}\right]}$ if, and only if, for all $\vec{s}, \vec{s}^{\prime}$ as above, and all $w \in W\left(G_{m}\right)$ that conjugate $\vec{s}$ to $\vec{s}^{\prime}$, we have $\phi_{\vec{s}}(x)=\psi_{w} \phi_{\vec{s}^{\prime}}(x)$.

Proof. It suffices to show that the action of $x$ on $i_{P_{v}}^{G_{m}} \otimes_{j} \mathcal{P}_{\nu_{j}} \otimes \overline{\mathcal{K}}$ coincides with an element of $A_{[L, \pi]} \otimes \overline{\mathcal{K}}$. Elements of the latter have the form $\prod_{M, \tilde{\pi}} A_{M, \tilde{\pi}}$, where $(M, \tilde{\pi})$ runs over a set of representatives for the inertial equivalence classes of pairs over $\overline{\mathcal{K}}$ with $\bmod \ell$ inertial supercuspidal support $\left(L_{m}, \pi_{m}\right)$.

For $\vec{s}$, let $M_{\vec{s}}$ be the product of the $M_{\vec{s}}$ and let $\pi_{\vec{s}}$ be the tensor product of the $\pi_{s_{j}}$. Then $\left(M_{\vec{s}}, \pi_{\vec{s}}\right)$ is inertially equivalent to a unique pair $(M, \tilde{\pi})$ as above. This inertial equivalence induces a map:

$$
A_{M, \tilde{\pi}} \rightarrow \bigotimes_{j} \overline{\mathcal{K}}\left[Z_{\vec{s}_{j}^{\prime}}\right]
$$


and the image consists of those elements that are invariant under all $w \in W\left(G_{m}\right)$ that conjugate $\vec{s}^{\prime}$ to itself.

Moreover, if $\vec{s}$ and $\vec{s}^{\prime}$ are such that $\left(M_{\vec{s}}, \pi_{\vec{s}}\right)$ and $\left(M_{\vec{s}^{\prime}}, \pi_{\vec{s}^{\prime}}\right)$ are both inertially equivalent to $(M, \tilde{\pi})$, then there exists a $w \in W\left(G_{m}\right)$ that conjugates $\vec{s}$ to $\vec{s}^{\prime}$, and then the diagram:

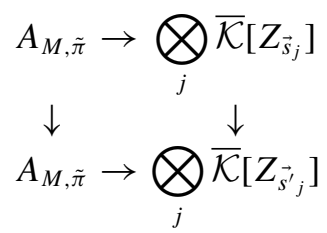

commutes, where the left vertical arrow is the identity and the right is $\psi_{w}$.

Thus, if $x$ satisfies the hypothesis of the theorem, it gives rise to a well-defined element of $A_{\left[L_{m}, \pi_{m}\right]} \otimes \overline{\mathcal{K}}$, and the action of this element on $i_{P_{v}}^{G} \otimes_{j} \mathcal{P}_{v_{j}} \otimes \overline{\mathcal{K}}$ coincides with that of $x$. Thus $x$ lies in the image of $A_{\left[L_{m}, \pi_{m}\right]}$ as claimed.

EXAMPLE 13.9. Suppose that $e_{q^{f^{\prime}}}>m$ and so the partition $v$ consists entirely of 1's. (This includes, in particular, any banal situation.) The ring $E_{1}$ is the ring $W(k)\left[\Theta_{1,1}^{ \pm 1}\right]$. An element $x$ of $E_{1}^{\otimes m}$ satisfies the conditions of Theorem 13.8 if, and only if $x$ is invariant under $S_{m}$. Thus in this case $A_{[L, \pi]}$ is simply an algebra of symmetric polynomials; in fact, one has $C_{[L, \pi]}=A_{\left[L_{m}, \pi_{m}\right]}$ in this case.

EXAMPLE 13.10. Suppose that $\ell>m$, and that $q^{f^{\prime}} \cong 1$ modulo $\ell$. Then the partition $v$ consists entirely of 1 's, and $E_{1}$ is isomorphic to $W(k)\left[\Theta_{1,1}^{ \pm 1}, \zeta\right] / \zeta^{\ell^{a}}-1$, where $\ell^{a}$ is the largest power of $\ell$ dividing $q-1$. Then, as in the last example, an element of $x$ in $E_{1}^{\otimes m}$ satisfies the conditions of Theorem 13.8 if, and only if $x$ is $S_{m}$ invariant. We thus obtain an explicit description of $A_{\left[L_{m}, \pi_{m}\right]}$ in this setting as well. (Note that this case includes - but is slightly larger than - what is often called the 'quasibanal' setting in the literature.)

EXAMPLE 13.11. Suppose that $e_{q f^{\prime}} \leqslant m<2 e_{q^{f^{\prime}}}$, so that the partition $v$ is given by $1, \ldots, 1, e_{q^{f^{\prime}}}$. The ring $E_{1}$ is given by $W(k)\left[\Theta_{1,1}^{ \pm 1}\right]$, and, by Proposition 9.9, $E_{e_{q^{\prime}}}$ is given by:

$$
E_{e_{q^{f^{\prime}}}}=E_{\sigma_{e_{q} f^{\prime}}}\left[\Theta_{1, e_{q f^{\prime}}}, \ldots, \Theta_{e_{q f^{\prime}}, e_{q^{f^{\prime}}}}, \Theta_{e_{q^{f^{\prime}}, e_{q} f^{\prime}}}^{-1}\right] / I^{\text {cusp }} \cdot\left\langle\Theta_{1, e_{q} f^{\prime}}, \ldots, \Theta_{e_{q f^{\prime}}-1, e_{q^{\prime}}}\right\rangle .
$$

(Recall that $I^{\text {cusp }}$ is the kernel of the map $E_{\sigma_{e} f^{\prime}} \rightarrow W(k)$ giving the action of $E_{\sigma_{e^{f^{\prime}}}}$ on the unique irreducible, noncuspidal summand of $\mathcal{P}_{\sigma_{e^{f^{\prime}}}} \otimes \overline{\mathcal{K}}$.)

We regard $E_{\sigma_{e} f^{\prime}}$ as a subalgebra of $E_{1}^{\otimes m-e_{q} f^{\prime}} \otimes E_{e_{q f^{\prime}}}$ by the inclusion $E_{\sigma_{e^{f^{\prime}}}} \rightarrow E_{e_{q f^{\prime}}}$. It is then not hard to verify, using Theorem 13.8, that the image of 
$A_{[L, \pi]}$ in $E_{1}^{\otimes m-e_{q} f} \otimes E_{e_{q^{\prime}}}$ is generated by $E_{\sigma_{e^{f^{\prime}}}}$, together with the images of the elements $\Theta_{1}, \ldots, \Theta_{m}, \Theta_{m}^{-1}$ of $A_{[L, \pi]}$. (First one proves these elements generate after tensoring with $\overline{\mathcal{K}}$, then one verifies that if $\ell x$ is in the subalgebra generated by these elements, then so is $x$.)

This yields a presentation:

$$
A_{\left[L_{m}, \pi_{m}\right]} \cong E_{\sigma_{e_{q} f}}\left[\Theta_{1}, \ldots, \Theta_{m}, \Theta_{m}^{-1}\right] / I^{\text {cusp }} \cdot J
$$

where $J$ is the ideal generated by the elements: $\Theta_{j}$ for $m-e_{q f^{\prime}}+1 \leqslant j \leqslant e_{q^{f^{\prime}}}-1$ and $\Theta_{j}-\Theta_{e_{q} f^{\prime}} \Theta_{j-e_{q} f^{\prime}}$ for $e_{q^{f^{\prime}}}<j \leqslant m$. As remarked earlier, Paige's description of the structure of $E_{\sigma_{e^{f^{\prime}}}}[\mathrm{Pg}]$ means that this presentation is completely explicit.

In general, when $v$ is a more complicated partition, the criteria of Theorem 13.8 do not realize $A_{\left[L_{m}, \pi_{m}\right]}$ as a ring of invariants in $\bigotimes_{j} E_{v_{j}}$. Obtaining a clean description of $A_{\left[L_{m}, \pi_{m}\right]}$ in this setting is substantially more complicated; in particular it is unlikely that one can find a clean presentation like the ones above. We refer the reader to [H2], where we will address this question from a rather different perspective.

\section{Acknowledgments}

The author is grateful to Matthew Emerton, Richard Taylor, Sug-Woo Shin, Florian Herzig, David Paige and J.-F. Dat for helpful conversations and encouragement on the subject of this note, and also to an anonymous referee who suggested many improvements to the exposition, and a strengthening of Theorem 12.4. This paper was partially supported by NSF grant DMS-1161582, and partially by EPSRC grant EP/M029719/1.

Throughout this paper the author works with the base ring $W(k)$. He thus adopts the convention that all tensor products are taken over $W(k)$ unless otherwise specified.

\section{References}

[BD] J. Bernstein and P. Deligne, 'Le 'centre' de Bernstein', in Representations des groups redutifs sur un corps local, Traveaux en cours (ed. P. Deligne) (Hermann, Paris, 1984), $1-32$.

[BR] J. Bernstein and K. Rumelhart, Representations of p-adic Groups, Lecture Notes (Harvard University, 1992).

[BZ] J. Bernstein and A. Zelevinski, 'Induced representations of p-adic groups I', Ann. Sci. Éc. Norm. Supér. (4) 10(4) (1977), 441-472. 
[B] C. Bushnell, 'Representations of reductive $p$-adic groups: localization of Hecke algebras and applications', J. Lond. Math. Soc. (2) 63(2) (2001), 364-386.

$[\mathrm{BH}]$ C. Bushnell and G. Henniart, 'Generalized Whittaker models and the Bernstein center', Amer. J. Math. 125(3) (2003), 513-547.

[BK1] C. Bushnell and P. Kutzko, The Admissible Dual of GL(N) Via Compact Open Subgroups, Annals of Mathematics Studies, 129 (Princeton University Press, Princeton, 1993).

[BK2] C. Bushnell and P. Kutzko, 'Smooth representations of reductive $p$-adic groups: structure theory via types', Proc. Lond. Math. Soc. (3) 77 (1998), 582-634.

[BK3] C. Bushnell and P. Kutzko, 'Semisimple types in $\mathrm{GL}_{n}$ ', Compositio Math. 119 (1999), 53-97.

[CE] M. Cabanes and M. Enguehard, Representation Theory of Finite Reductive Groups, New Mathematical Monographs, 1 (Cambridge University Press, Cambridge, 2004).

[D1] J.-F. Dat, 'Caractières à valeurs dans le centre de Bernstein', J. Reine Angew. Math. 508 (1999), 61-83.

[D2] J.-F. Dat, 'Integral structures in Bernstein's center', Preprint, 2006.

[D3] J.-F. Dat, 'Finitude pour les représentations lisses de groupes p-adiques', J. Inst. Math. Jussieu 8(2) (2009), 261-333.

[D4] J.-F. Dat, 'Théorie de Lubin-Tate non abélienne $\ell$-entière', Duke Math. J. 161(6) (2012), 951-1010.

[EH] M. Emerton and D. Helm, 'The local Langlands correspondence for $\mathrm{GL}_{n}$ in families', Ann. Sci. Éc. Norm. Supér. (4) 47(4) (2014), 655-722.

[H1] D. Helm, 'Whittaker models and the integral Bernstein center for $\mathrm{GL}_{n}(F)$ ', Duke. Math. J. (to appear). Preprint, arXiv:1210.1789.

[H2] D. Helm, 'Curtis homomorphisms and the integral Bernstein center for $\mathrm{GL}_{n}(F)$ ', (to appear). Preprint, arXiv:1605.00487.

[HM] D. Helm and G. Moss, 'Converse theorems and the local Langlands correspondence in families', (in preparation).

[HL] R. B. Howlett and G. I. Lehrer, 'On Harish-Chandra induction and restriction for modules of Levi subgroups', J. Algebra 165(1) (1994), 172-183.

[Ja] G. James, 'The irreducible representations of the finite general linear groups', Proc. Lond. Math. Soc. (3) 52(2) (1986), 236-268.

[Lu] G. Lusztig, 'Affine Hecke algebras and their graded version', J. Amer. Math. Soc. 2 (1989), 599-635.

[Pg] D. Paige, 'The projective envelope of a cuspidal representation of a finite linear group', J. Number Theory 136 (2014), 354-374.

[Pa] V. Paskunas, 'The image of Colmez's Montreal functor', Publ. Math. Inst. Hautes Études Sci. 188 (2013), 1-191.

[R] A. Roche, 'Parabolic induction and the Bernstein decomposition', Compositio Math. 134(2) (2002), 113-133.

[V1] M.-F. Vigneras, Representations l-modulaires d'un groupe réductif $p$-adique avec $l \neq p$, Progress in Mathematics, 137 (Birkhauser, Boston, 1996).

[V2] M.-F. Vigneras, 'Induced $R$-representations of $p$-adic reductive groups', Selecta Math. 4(4) (1998), 549-623.

[V3] M.-F. Vigneras, 'Correspondance de Langlands semi-simple pour $\operatorname{GL}(n, F)$ modulo $\ell \neq p$, Invent. Math. 144(1) (2001), 177-223. 